Supporting Information for

\title{
2-Bromo-6-isocyanopyridine as a Universal Convertible Isocyanide for Multicomponent Chemistry
}

Gydo van der Heijden, J.A.W. (Sjaak) Jong, Eelco Ruijter*, and Romano V.A. Orru*

\begin{abstract}
*Department of Chemistry and Pharmaceutical Sciences, Amsterdam Institute for Molecules, Medicines \& Systems, VU University Amsterdam, De Boelelaan 1083, NL-1081 HV Amsterdam, The Netherlands.
\end{abstract}

E-mail:

e.ruijter@vu.nl

r.v.a.orru@vu.nl 


\section{Contents}

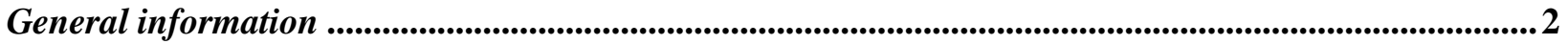

Optimization data ..........................................................................................................................................3

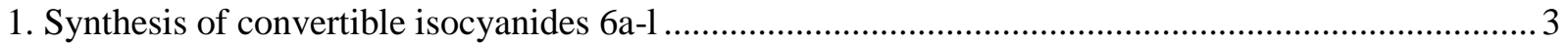

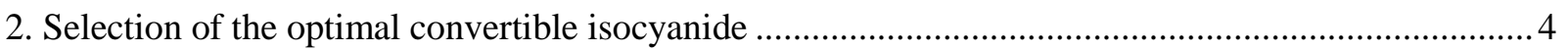

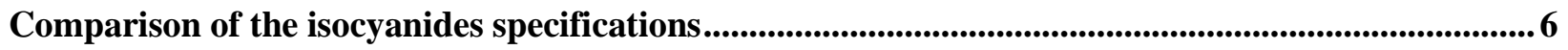

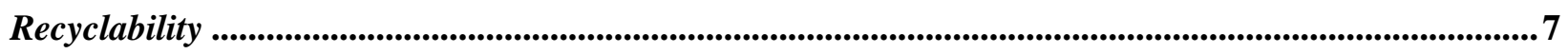

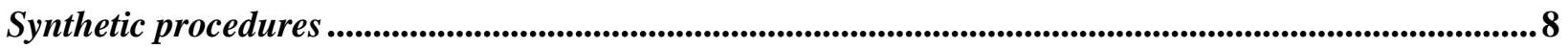

General procedure I: Synthesis of 2-formamidopyridines (S1) f.................................................... 8

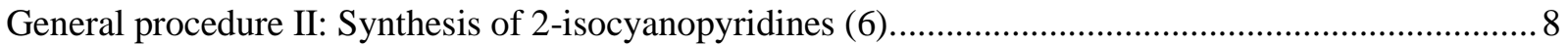

General procedure III: Synthesis of 2-(N-benzylacetamido)-N-(pyridin-2-yl)-4-methylpentan-amides (7)

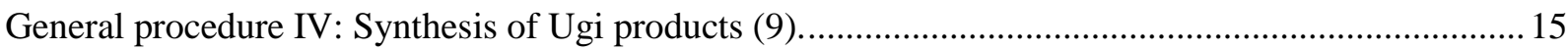

General procedure V: Hydrolysis of Ugi products. ........................................................................... 19

General procedure VI: Synthesis of Passerini products (11) .......................................................2

General procedure VII: Hydrolysis of the Passerini products. .............................................................24

General procedure VIII: Transacylation under basic conditions. ........................................................24

General procedure IX: Transacylation under acidic conditions..........................................................25

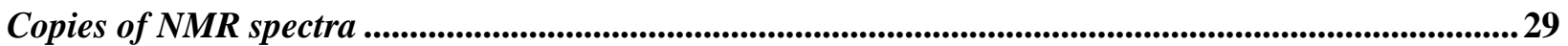

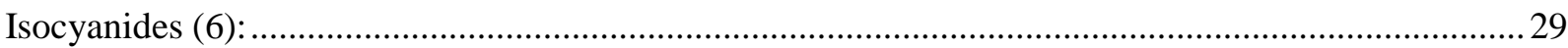

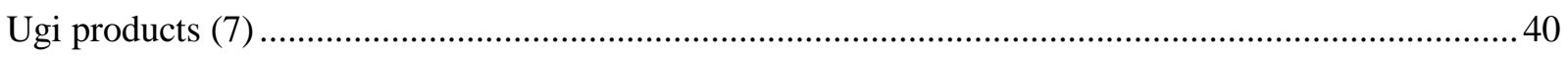

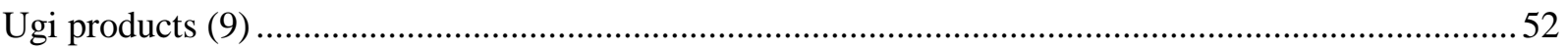

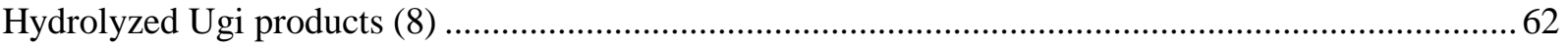

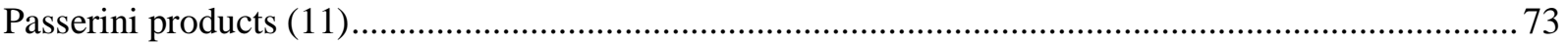

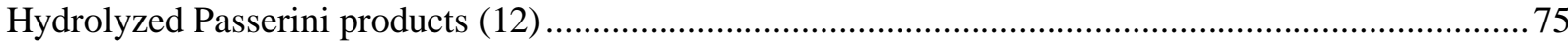

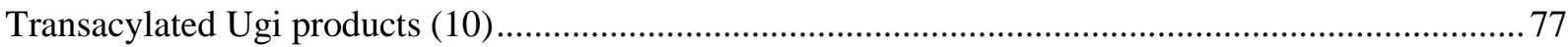

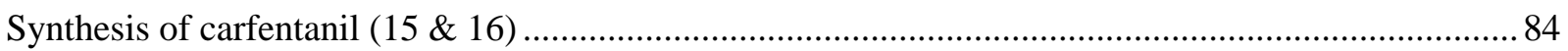




\section{$\underline{\text { General information }}$}

Unless stated otherwise, all solvents and commercially available reagents were used as purchased. Powder $3 \AA$ and $4 \AA$ molecular sieves were purchased from Sigma Aldrich and activated before use. GC yield and conversion analysis was performed using a Shimadzu GC2010 equipped with a Zebron ZB-1 capillary column $(30 \mathrm{~m} \times 0.25 \mathrm{~mm})$ with dodecane as internal standard. Infrared (IR) spectra were recorded neat using a Shimadzu FTIR-8400s spectrophotometer and wavelengths are reported in $\mathrm{cm}^{-1}$. Nuclear magnetic resonance (NMR) spectra were recorded on a Bruker Avance $500\left(125.78 \mathrm{MHz}\right.$ for $\left.{ }^{13} \mathrm{C}\right)$ or Bruker Avance $400\left(100.62 \mathrm{MHz}\right.$ for $\left.{ }^{13} \mathrm{C}\right)$ using the residual solvent as internal $\left({ }^{1} \mathrm{H}\right.$ : $\delta 7.26 \mathrm{ppm}$, ${ }^{13} \mathrm{C}\left\{{ }^{1} \mathrm{H}\right\}: \delta 77.16 \mathrm{ppm}$ for $\mathrm{CDCl}_{3},{ }^{1} \mathrm{H}: \delta 2.50 \mathrm{ppm},{ }^{13} \mathrm{C}\left\{{ }^{1} \mathrm{H}\right\}: \delta 39.52 \mathrm{ppm}$ for DMSO-d $\mathrm{d}_{6}$ and ${ }^{1} \mathrm{H}: \delta 3.31$ ppm, ${ }^{13} \mathrm{C}\left\{{ }^{1} \mathrm{H}\right\}: \delta 49.00 \mathrm{ppm}$ for MeOD- $\left.\mathrm{d}_{4}\right)$. Chemical shifts $(\delta)$ are given in ppm and coupling constants $(J)$ are quoted in hertz $(\mathrm{Hz})$. Resonances are described as s (singlet), d (doublet), t (triplet), q (quartet), bs (broad singlet) and $\mathrm{m}$ (multiplet) or combinations thereof. Melting points were recorded on a Büchi M565 melting point apparatus and are uncorrected. Electrospray Ionization (ESI) high-resolution mass spectrometry was carried out using a Bruker micrOTOF-Q instrument in positive ion mode (capillary potential of $4500 \mathrm{~V}$ ). Flash chromatography was performed on Silicycle Silia-P Flash Silica Gel (particle size $40-63 \mu \mathrm{m}$, pore diameter $60 \AA$ ) using the indicated eluent. Thin Layer Chromatography (TLC) was performed using TLC plates from Merck (SiO2, Kieselgel 60 F254 neutral, on aluminium with fluorescence indicator) and compounds were visualized by UV detection (254 nm) and $\mathrm{KMnO}_{4}$ stain. 


\section{Optimization data}

\section{Synthesis of convertible isocyanides 6a-l}

A range of substituted 2-aminopyridines 5a-l were converted to the corresponding 2-isocyanopyridines S1a-l via the formamides 6a-l by standard chemical transformations (Scheme S1, Experimental part: General Procedures I and II).

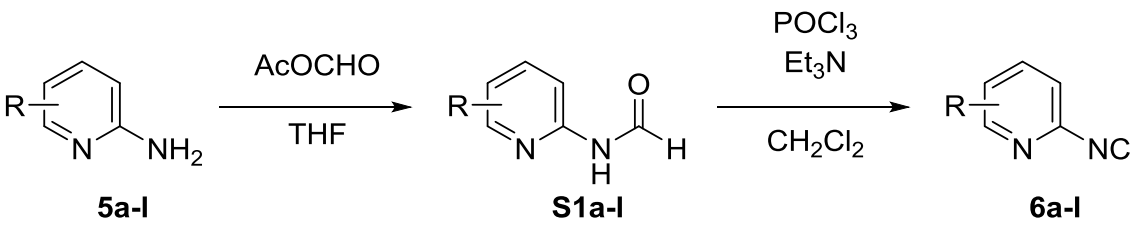

Scheme S1. Synthesis of substituted 2-isocyanopyridines (6a-1).

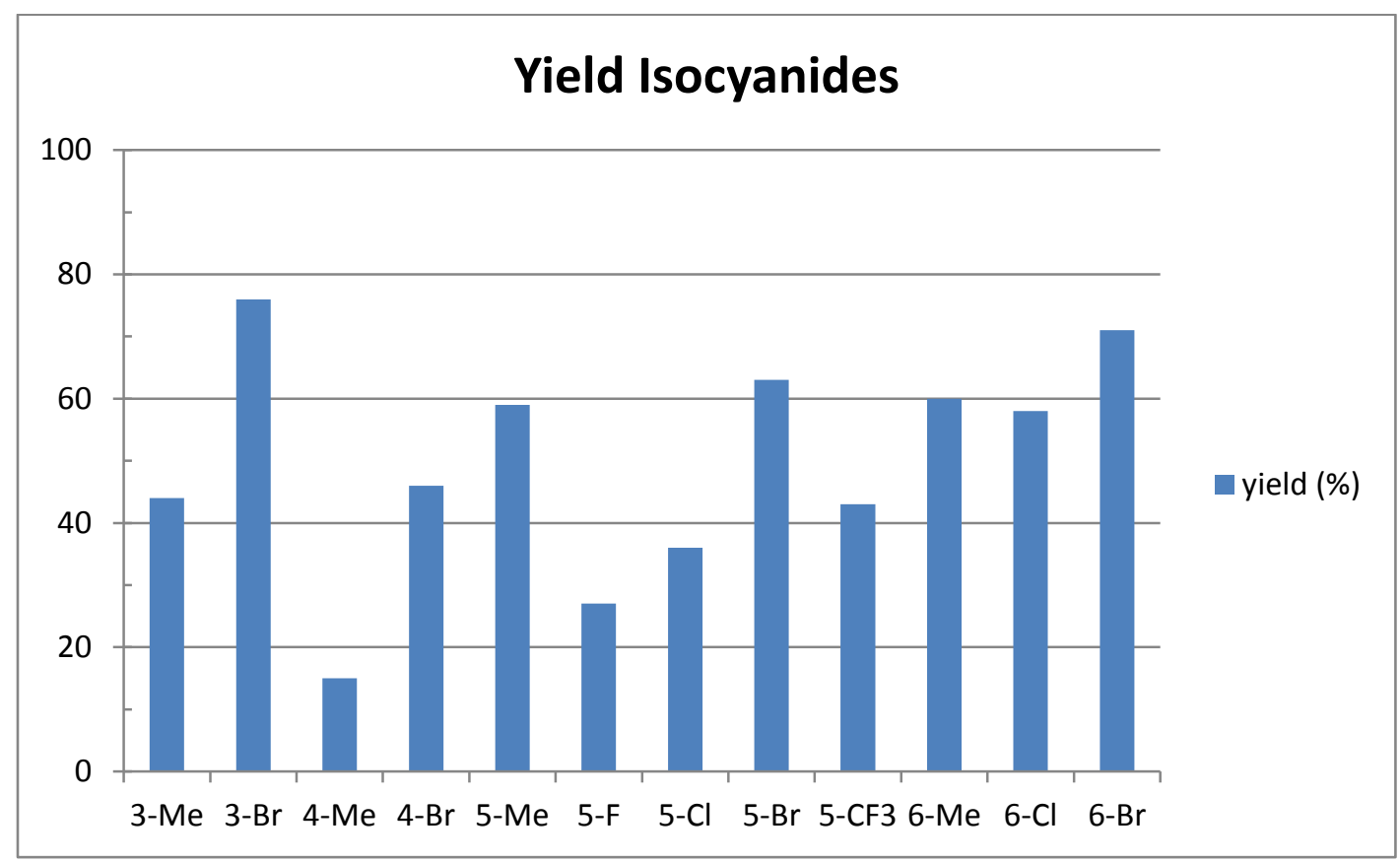

Figure S1. Yields of various substituted 2-isocyanopyridines 6a-l from the corresponding amines over two steps.

For most aminopyridines, the transformations proceeded in moderate to good yields (see Figure S1). In the case of 2-amino-5-nitropyridine, 2-amino-5-cyanopyridine and 2-aminopyrimidine (not depicted in figure S1) the corresponding isocyanide was too electron-deficient, resulting in a very unstable isocyanide. 


\section{Selection of the optimal convertible isocyanide}

The synthesized 2-isocyanopyridines 6a-l were then used in the benchmark Ugi reaction with isovaleraldehyde, benzylamine and acetic acid (Scheme S2, Experimental part: General Procedures III) to give Ugi products $7 \mathbf{a}-\mathbf{l}$, which were then subjected to hydrolysis $\left(2.5\right.$ or 5.0 equiv $\mathrm{NaOH}, \mathrm{MeOH} / \mathrm{H}_{2} \mathrm{O}, 48$ $\mathrm{h}, \mathrm{rt}$ ). The conversion to the corresponding carboxylic acid 8a was monitored by GC. The yields for the Ugi reaction and the subsequent hydrolysis for various convertible isocyanides 6a-l are depicted in Figures S2 and S3, respectively.
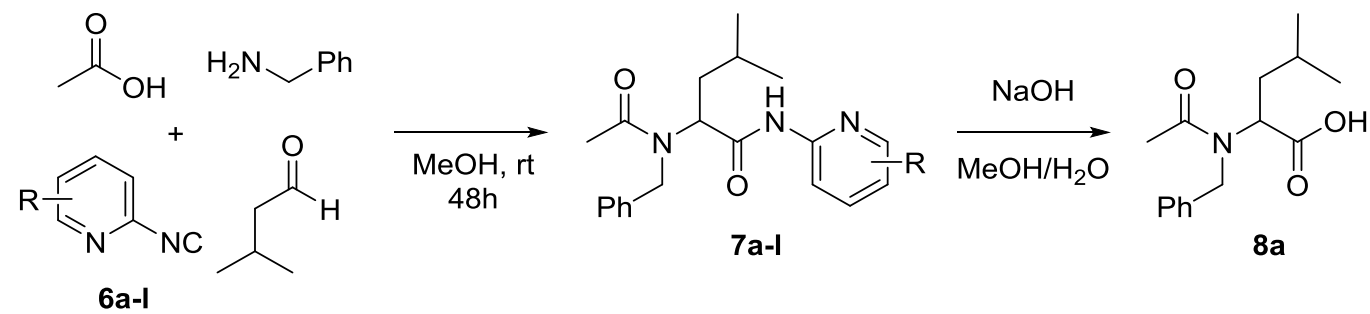

Scheme S2. Benchmark Ugi reaction with various substituted 2-isocyanopyridines (6a-l) and subsequent hydrolysis

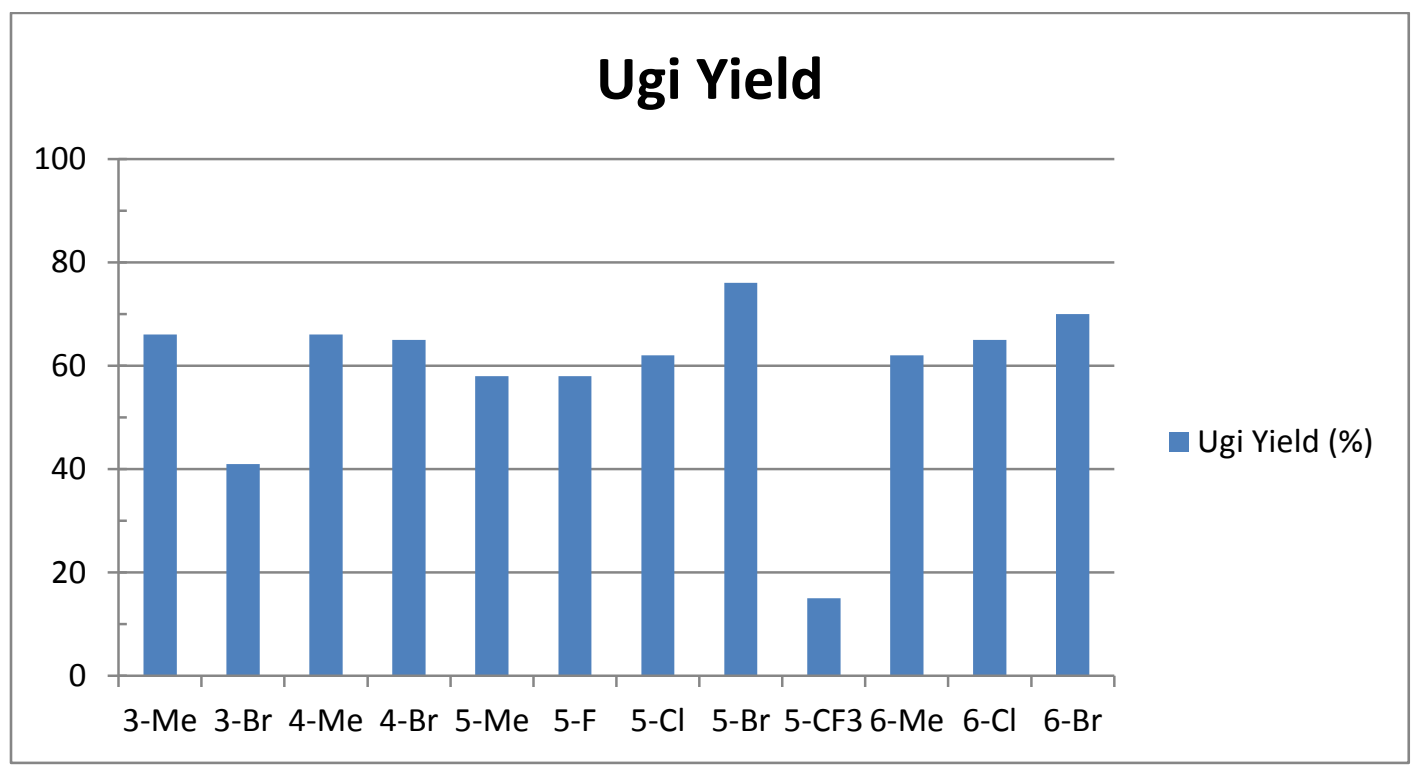

Figure S2. Yields of benchmark Ugi reactions with various substituted 2-isocyanopyridines (6a-I) 


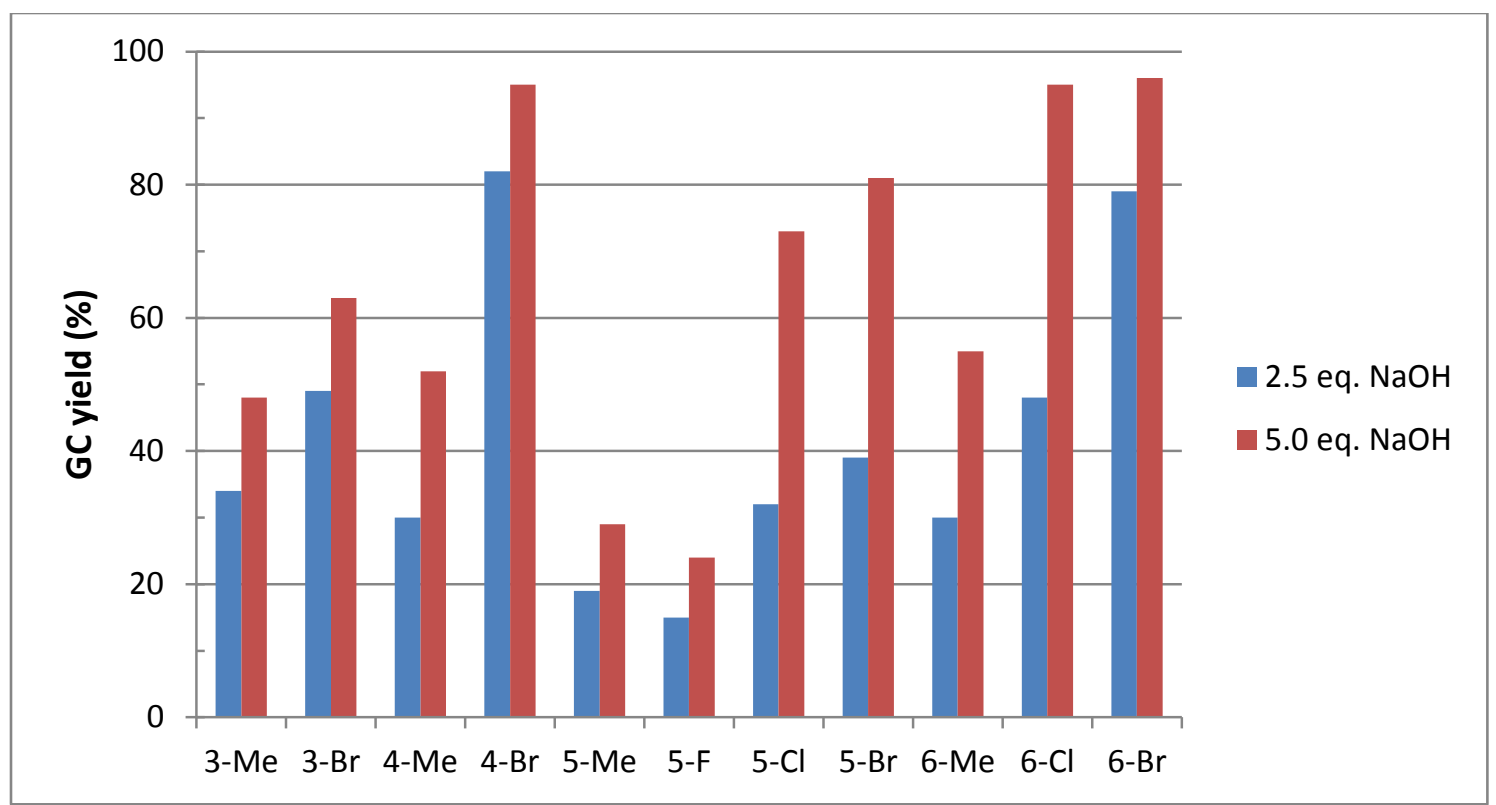

Figure S3. Yields of hydrolysis of Ugi products 7a-l using 2.5 equiv or 5.0 equiv of $\mathrm{NaOH}$ in $\mathrm{MeOH} / \mathrm{H}_{2} \mathrm{O}$.

The results in Figures S2 and S3 clearly indicate that isocyanide with $\mathrm{R}=6-\mathrm{Br}(\mathbf{6 1})$ is most effective in both the Ugi reaction and subsequent hydrolysis, and can be produced in relatively high yield from the corresponding amine (Figure S1). We therefore selected this convertible isocyanide as the optimal candidate. 


\section{Comparison of isocyanide specifications}

Table S1: Comparison of the specifications of current convertible isocyanides compared to $6 \mathbf{1}$.

\begin{tabular}{|c|c|c|c|c|c|c|c|}
\hline & $\begin{array}{l}\text { (a) The } \\
\text { isocyanides } \\
\text { require } \\
\text { lengthy, } \\
\text { tedious } \\
\text { synthetic } \\
\text { routes }^{a}\end{array}$ & $\begin{array}{l}\text { (b) The } \\
\text { isocyanides } \\
\text { themselves are } \\
\text { instable/difficult } \\
\text { to handle }\end{array}$ & $\begin{array}{l}\text { (c) The } \\
\text { conversion to } \\
\text { different } \\
\text { functional } \\
\text { groups is } \\
\text { limited }\end{array}$ & $\begin{array}{l}\text { (d) Cleavage of } \\
\text { the convertible } \\
\text { isocyanides } \\
\text { requires a } \\
\text { multistep } \\
\text { transformation }\end{array}$ & $\begin{array}{l}\text { (e) The MCR } \\
\text { and the } \\
\text { conversion } \\
\text { can be } \\
\text { performed in } \\
\text { one-pot }\end{array}$ & $\begin{array}{l}\text { (f) The } \\
\text { isocyanide } \\
\text { is } \\
\text { recyclable } \\
\text { after } \\
\text { cleavage }\end{array}$ & $\begin{array}{l}\text { (g) conditions } \\
\text { for cleavage }\end{array}$ \\
\hline & & & & & & & Acidic \\
\hline & & & & & & & Basic \\
\hline $\mathrm{R}$ & & & & & & & Acidic \\
\hline OTBS & & & & & & & Acidic \\
\hline $\mathrm{O}_{2}$ & & & & & & $b$ & Basic \\
\hline $\mathrm{OMe}$ & & & & & & & Multistep \\
\hline $\mathrm{NO}_{2}$ & & & & & & & Multistep \\
\hline NC & & & & & & & Multistep \\
\hline $\begin{array}{ll}\mathrm{Br}^{-} & \mathrm{N}\end{array}$ & & & & & & & $\begin{array}{l}\text { Acidic or } \\
\text { basic }\end{array}$ \\
\hline
\end{tabular}

${ }^{a}$ More than two reaction steps is considered as lengthy. ${ }^{b}$ The isocyanide has the potential to be recycled. 


\section{$\underline{\text { Recyclability }}$}

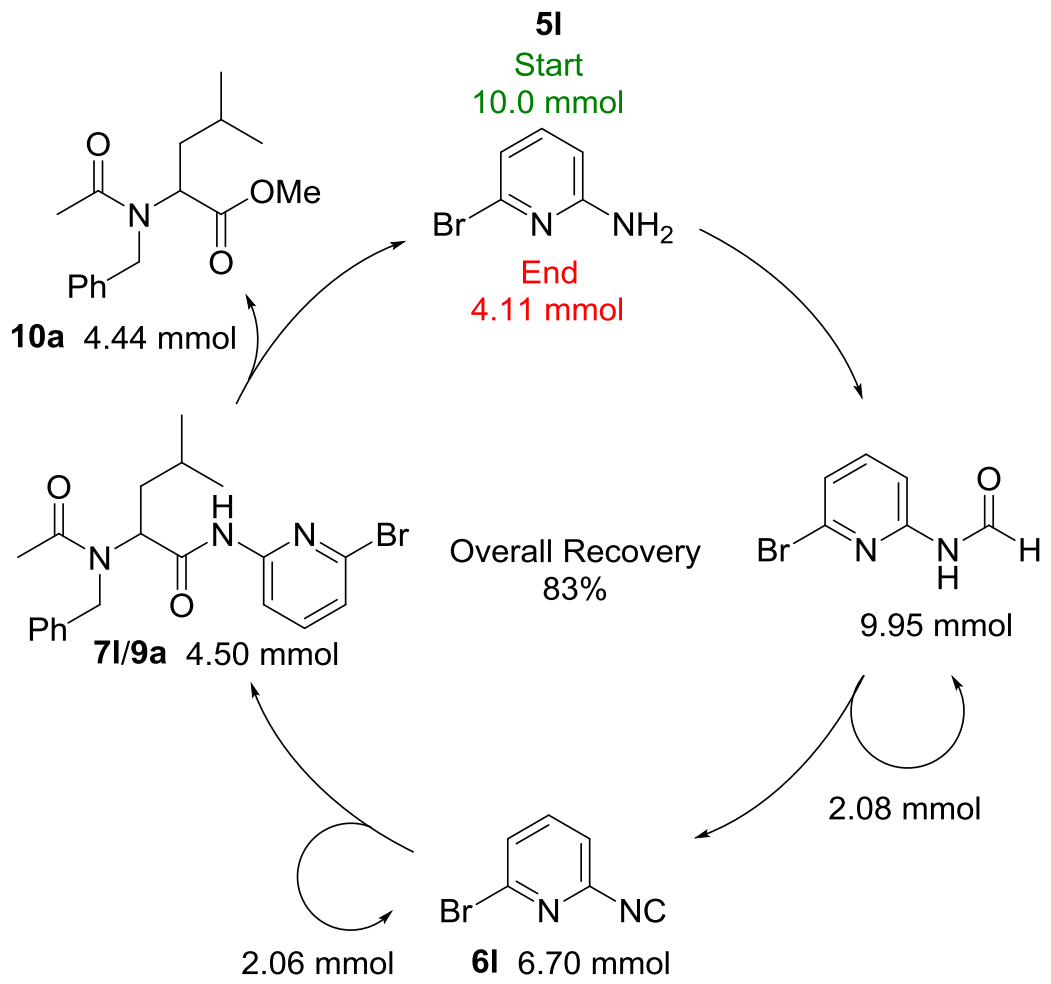

Scheme S3: Recyclability of 2-bromo-6-isocyanopyridine (5l). 


\section{Synthetic procedures}

\section{General procedure I: Synthesis of 2-formamidopyridines (S1).}

Unless stated otherwise: In a flame-fried roundbottom flask equipped with a reflux condenser formic acid (41 mmol, 2.05 equiv) was added dropwise to acetic anhydride ( $40 \mathrm{mmol}, 2.0$ equiv) and the resulting mixture refluxed at $65{ }^{\circ} \mathrm{C}$ for 2-3 hours. The mixture was cooled to room temperature and slowly added to a cold solution of a 2-aminopyridine $\left(\mathbf{5}, 20 \mathrm{mmol}, 1.0\right.$ equiv) in anhydrous THF $(50 \mathrm{~mL})$ at $0{ }^{\circ} \mathrm{C}$. After 1 $\mathrm{h}$, the reaction was allowed to warm up to rt and stirred for 2-3 more hours. The solvent and excess of acetic acid were evaporated and the residue was redissolved in EtOAc. The crude mixture was washed with a saturated $\mathrm{NaHCO}_{3}$ and the water layer was extracted with EtOAc (2x). The organic layers were combined, washed with brine and dried over $\mathrm{Na}_{2} \mathrm{SO}_{4}$. The solvent was evaporated to furnish the corresponding formamide $\mathbf{S 1}$.

\section{$N$-(6-bromopyridin-2-yl)formamide (S11, 13).}

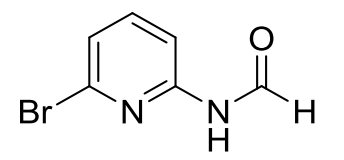

Following general procedure I on a 300 mmol scale with 2-amino-6bromopyridine (51, $51.9 \mathrm{~g}, 300 \mathrm{mmol}, 1.0$ equiv) the formamide $\mathbf{S 1 I}$ was obtained as a white solid (59.9 g, $298 \mathrm{mmol}$, 99\%). Two rotamers were present on NMR timescale $\left(\mathrm{R}^{1}: \mathrm{R}^{2}=1: 0.5\right)$. m.p.: $140-144{ }^{\circ} \mathrm{C} .{ }^{1} \mathrm{H}$ NMR $\left(\mathrm{CDCl}_{3}, 500 \mathrm{MHz}\right) \delta 9.32$ $(\mathrm{d}, J=10.5 \mathrm{~Hz}, 0.5 \mathrm{H}), 8.85(\mathrm{bs}, 0.5 \mathrm{H}), 8.71(\mathrm{bs}, 1 \mathrm{H}), 8.53(\mathrm{~s}, 1 \mathrm{H}), 8.19(\mathrm{~d}, J=8.0 \mathrm{~Hz}, 1 \mathrm{H}), 7.59(\mathrm{t}, J=$ $8.0 \mathrm{~Hz}, 1 \mathrm{H}), 7.51(\mathrm{t}, J=8.0 \mathrm{~Hz}, 0.5 \mathrm{H}), 7.27-7.23(\mathrm{~m}, 1.5 \mathrm{H}), 6.83(\mathrm{~d}, J=8.0 \mathrm{~Hz}, 0.5 \mathrm{H}) .{ }^{13} \mathrm{C} \mathrm{NMR}\left(\mathrm{CDCl}_{3}\right.$, $125 \mathrm{MHz}) \delta 162.6(\mathrm{CH}), 159.4(\mathrm{CH}), 150.7\left(\mathrm{C}_{\mathrm{q}}\right), 150.6\left(\mathrm{C}_{\mathrm{q}}\right), 140.9(\mathrm{CH}), 140.5(\mathrm{CH}), 139.2\left(\mathrm{C}_{\mathrm{q}}\right), 124.1$ $(\mathrm{CH}), 123.7(\mathrm{CH}), 113.4(\mathrm{CH}), 109.3(\mathrm{CH})$. IR (neat): vmax $\left(\mathrm{cm}^{-1}\right)=3055(\mathrm{w}), 1686(\mathrm{~s}), 1562(\mathrm{~s}), 1439$ (s), 1397 (s), 1294 (s), 1238 (m), 1157 (s), 1132 (s), 792 (m), 715 (s), 517 (s). HRMS (ESI): m/z calculated for $\mathrm{C}_{6} \mathrm{H}_{6} \mathrm{BrN}_{2} \mathrm{O}[\mathrm{M}+\mathrm{H}]^{+} 200.9658$, found 200.9656 .

\section{General procedure II: Synthesis of 2-isocyanopyridines (6).}

Unless stated otherwise: To a flame dried 3-neck flask a 2-formamidepyridine (S1, 10 mmol, 1.0 equiv) was added and dissolved in anhydrous $\mathrm{CH}_{2} \mathrm{Cl}_{2}(30 \mathrm{~mL})$. Then, $\mathrm{Et}_{3} \mathrm{~N}(60 \mathrm{mmol}, 6.0$ equiv) was added and the solution was cooled to $-78{ }^{\circ} \mathrm{C}$. $\mathrm{POCl}_{3}\left(11.5 \mathrm{mmol}, 1.15\right.$ equiv) in anhydrous $\mathrm{CH}_{2} \mathrm{Cl}_{2}(5 \mathrm{~mL})$ was added dropwise to the mixture. After $1 \mathrm{~h}$, the mixture was warmed up to $0{ }^{\circ} \mathrm{C}$ and stirred overnight. The crude reaction mixture was carefully quenched with saturated $\mathrm{NaHCO}_{3}(25 \mathrm{~mL})$. The layers were separated and the water layer was extracted with $\mathrm{CH}_{2} \mathrm{Cl}_{2}(2 \times)$. The organic layers were combined and washed with brine, dried over $\mathrm{Na}_{2} \mathrm{SO}_{4}$ and concentrated. The residue was purified by flash chromatography $\left(\mathrm{CH}_{2} \mathrm{Cl}_{2}\right)$ to afford the 2-isocyanopiridine $\mathbf{6}$. The product was stored in the freezer at $-18^{\circ} \mathrm{C}$.

\section{2-Isocyano-3-methylpyridine (6a).}

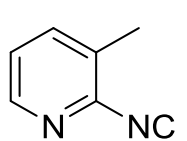

Following general procedure II with $N$-(3-methylpyridin-2-yl)formamide (S1a, 1.36 g, 10 mmol, 1.0 equiv) the isocyanide was obtained as an off-white crystalline solid (520 $\mathrm{mg}, 4.4$

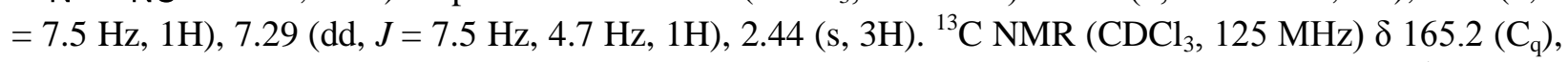
147.0 $(\mathrm{CH}), 140.3\left(\mathrm{C}_{\mathrm{q}}\right), 139.4(\mathrm{CH}), 130.3\left(\mathrm{C}_{\mathrm{q}}\right), 124.6(\mathrm{CH}), 17.9\left(\mathrm{CH}_{3}\right)$. IR (neat): vmax $\left(\mathrm{cm}^{-1}\right)=3053$ 
(w), 2122 (s), 1572 (s), 1115 (s), 804 (s), 633 (s), 534 (s), 498 (s), 413 (s), 401 (s). HRMS (ESI): m/z calculated for $\mathrm{C}_{7} \mathrm{H}_{7} \mathrm{~N}_{2} 119.0604[\mathrm{M}+\mathrm{H}]^{+}$, found 119.0609 .

\section{3-Bromo-2-isocyanopyridine (6b).}

$\mathrm{Br}$ Following general procedure II with $N$-(3-bromopyridin-2-yl)formamide (S1b, $2.01 \mathrm{~g}, 10$ mmol, 1.0 equiv) the isocyanide was obtained as a light-brown powder $(1.39 \mathrm{~g}, 7.6 \mathrm{mmol}$, 76\%). Degradation point: $64{ }^{\circ} \mathrm{C} .{ }^{1} \mathrm{H} \mathrm{NMR}\left(\mathrm{CDCl}_{3}, 500 \mathrm{MHz}\right) \delta 8.43(\mathrm{~d}, J=4.0 \mathrm{~Hz}, 1 \mathrm{H})$, $8.04(\mathrm{~d}, J=8.0 \mathrm{~Hz}), 7.29(\mathrm{dd}, J=8.0,4.8 \mathrm{~Hz}, 1 \mathrm{H}) .{ }^{13} \mathrm{C} \mathrm{NMR}\left(\mathrm{CDCl}_{3}, 125 \mathrm{MHz}\right) \delta 168.2$ $\left(\mathrm{C}_{\mathrm{q}}\right), 147.9(\mathrm{CH}), 142.0(\mathrm{CH}), 139.7\left(\mathrm{C}_{\mathrm{q}}\right), 125.6(\mathrm{CH}), 117.2\left(\mathrm{C}_{\mathrm{q}}\right)$. IR (neat): vmax $\left(\mathrm{cm}^{-1}\right)=3043(\mathrm{~s}), 2120$ (s), 1564 (s), 1416 (s), 1030 (s), 804 (s), 497 (s), 411 (s), 401 (s). HRMS (ESI): m/z calculated for $\mathrm{C}_{6} \mathrm{H}_{4} \mathrm{BrN}_{2}[\mathrm{M}+\mathrm{H}]^{+}$182.9552, found 182.9552 .

\section{2-Isocyano-4-methylpyridine (6c).}

Following general procedure II with $N$-(4-methylpyridin-2-yl)formamide (S1c, $1.36 \mathrm{~g}, 10$ mmol, 1.0 equiv) the isocyanide was obtained as a dark brown powder $(177 \mathrm{mg}, 1.5 \mathrm{mmol}$, 15\%). Degradation point: $51{ }^{\circ} \mathrm{C} .{ }^{1} \mathrm{H} \mathrm{NMR}\left(\mathrm{CDCl}_{3}, 500 \mathrm{MHz}\right) \delta 8.33(\mathrm{~d}, J=4.7 \mathrm{~Hz}, 1 \mathrm{H})$, $\mathrm{N} \mathrm{NC} \quad 7.19-7.18(\mathrm{~m}, 2 \mathrm{H}), 2.40(\mathrm{~s}, 3 \mathrm{H}) \cdot{ }^{13} \mathrm{C} \mathrm{NMR}\left(\mathrm{CDCl}_{3}, 125 \mathrm{MHz}\right) \delta 163.2\left(\mathrm{C}_{\mathrm{q}}\right), 150.6\left(\mathrm{C}_{\mathrm{q}}\right)$, $149.3(\mathrm{CH}), 140.4\left(\mathrm{C}_{\mathrm{q}}\right), 125.7(\mathrm{CH}), 121.9(\mathrm{CH}), 20.8\left(\mathrm{CH}_{3}\right)$. IR (neat): vmax $\left(\mathrm{cm}^{-1}\right)=3053(\mathrm{w}), 2122(\mathrm{~s})$, 1603 (s), 1404 (s), 837 (s), 496 (s), 451 (s), 413 (s), 401 (s). HRMS (ESI): $m / z$ calculated for $\mathrm{C}_{7} \mathrm{H}_{7} \mathrm{~N}_{2}[\mathrm{M}+$ $\mathrm{H}]^{+} 119.0604$, found 119.0613 .

\section{4-Bromo-2-isocyanopyridine (6d).}

$\mathrm{Br} \quad$ Following general procedure II with $N$-(4-bromopyridin-2-yl)formamide (S1d, $2.01 \mathrm{~g}, 10$ mmol, 1.0 equiv) the isocyanide was obtained as a light brown powder $(842 \mathrm{mg}, 4.6 \mathrm{mmol}$, 46\%). Degradation point: $76{ }^{\circ} \mathrm{C} .{ }^{1} \mathrm{H} \mathrm{NMR}\left(\mathrm{CDCl}_{3}, 500 \mathrm{MHz}\right) \delta 8.33(\mathrm{~d}, J=5.0 \mathrm{~Hz}, 1 \mathrm{H})$, $\mathrm{N} N \mathrm{NC} 7.56(\mathrm{~d}, J=6.6 \mathrm{~Hz}, 1 \mathrm{H}), 7.54(\mathrm{~s}, 1 \mathrm{H}) .{ }^{13} \mathrm{C} \mathrm{NMR}\left(\mathrm{CDCl}_{3}, 125 \mathrm{MHz}\right) \delta 165.7\left(\mathrm{C}_{\mathrm{q}}\right), 150.2$ $(\mathrm{CH}), 140.6\left(\mathrm{C}_{\mathrm{q}}\right), 134.5\left(\mathrm{C}_{\mathrm{q}}\right), 128.2(\mathrm{CH}), 124.7(\mathrm{CH})$. IR (neat): vmax $\left(\mathrm{cm}^{-1}\right)=3075(\mathrm{w}), 2133(\mathrm{~s}), 1564$ (s), 1381 (s), 837 (s), 633 (s), 498 (s), 451 (s), 418 (s), 401 (s). HRMS (ESI): $m / z$ calculated for $\mathrm{C}_{6} \mathrm{H}_{4} \mathrm{BrN}_{2}$ $[\mathrm{M}+\mathrm{H}]^{+}$182.9552, found 182.9555 .

\section{2-Isocyano-5-methylpyridine (6e).}

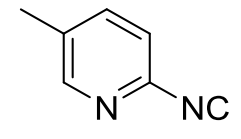

Following general procedure II with $N$-(5-methylpyridin-2-yl)formamide (S1e, $1.36 \mathrm{~g}$, $10 \mathrm{mmol}, 1.0$ equiv) the isocyanide was obtained as an orange-brown powder (697 $\mathrm{mg}$, 5.9 mmol, 59\%). M.p.: $62{ }^{\circ} \mathrm{C}$. Degradation point: $69{ }^{\circ} \mathrm{C} .{ }^{1} \mathrm{H} \mathrm{NMR}\left(\mathrm{CDCl}_{3}, 500 \mathrm{MHz}\right) \delta$ $8.29(\mathrm{~s}, 1 \mathrm{H}), 7.59(\mathrm{~d}, J=8.0 \mathrm{~Hz}, 1 \mathrm{H}), 7.24(\mathrm{~d}, J=8.1 \mathrm{~Hz}, 1 \mathrm{H}), 2.38(\mathrm{~s}, 3 \mathrm{H}) .{ }^{13} \mathrm{C} \mathrm{NMR}\left(\mathrm{CDCl}_{3}, 125 \mathrm{MHz}\right)$ $\delta 163.0\left(\mathrm{C}_{\mathrm{q}}\right), 149.9(\mathrm{CH}), 139.0(\mathrm{CH}), 138.1\left(\mathrm{C}_{\mathrm{q}}\right), 135.0\left(\mathrm{C}_{\mathrm{q}}\right), 120.7(\mathrm{CH}), 18.2\left(\mathrm{CH}_{3}\right)$. IR (neat): vmax $\left(\mathrm{cm}^{-1}\right)=3057(\mathrm{w}), 2127(\mathrm{~s}), 1578(\mathrm{~s}), 1474(\mathrm{~s}), 1034(\mathrm{~s}), 833(\mathrm{~s}), 633(\mathrm{~s}), 536(\mathrm{~s}), 525(\mathrm{~s}), 494(\mathrm{~s}), 413(\mathrm{~s})$, 401 (s). HRMS (ESI): $m / z$ calculated for $\mathrm{C}_{7} \mathrm{H}_{7} \mathrm{~N}_{2} 119.0604[\mathrm{M}+\mathrm{H}]^{+}$, found 119.0609 . 
5-Fluoro-2-isocyanopyridine (6f).<smiles>N#Cc1ccc(F)cn1</smiles>

Following general procedure II with $N$-(5-fluoropyridin-2-yl)formamide (S1f, $1.40 \mathrm{~g}$, $10 \mathrm{mmol}, 1.0$ equiv) the isocyanide was obtained as a dark brown powder $(0.330 \mathrm{~g}, 2.7$ mmol, 27\%). The product readily degrades at room temperature. Therefore, many impurities are visible in the ${ }^{1} \mathrm{H}-\mathrm{NMR}$. Degradation point: $67{ }^{\circ} \mathrm{C} .{ }^{1} \mathrm{H}$ NMR $\left(\mathrm{CDCl}_{3}, 500\right.$ $\mathrm{MHz}) \delta 8.35(\mathrm{~s}, 1 \mathrm{H}), 7.55-7.51(\mathrm{~m}, 1 \mathrm{H}), 7.41-7.36(\mathrm{~m}, 1 \mathrm{H})$.

\section{5-Chloro-2-isocyanopyridine (6g).}<smiles>[N-]=[N+]=Nc1ccc(Cl)cn1</smiles>

Following general procedure II with $N$-(5-chloropyridin-2-yl)formamide (S1g, 1.57 $\mathrm{g}, 10 \mathrm{mmol}, 1.0$ equiv) the isocyanide was obtained as a light brown powder (499 $\mathrm{mg}$, $3.6 \mathrm{mmol}, 36 \%)$. Degradation point: $86{ }^{\circ} \mathrm{C} .{ }^{1} \mathrm{H} \mathrm{NMR}\left(\mathrm{CDCl}_{3}, 500 \mathrm{MHz}\right) \delta 8.45(\mathrm{~s}, 1 \mathrm{H})$, $7.79(\mathrm{~d}, J=8.1 \mathrm{~Hz}, 1 \mathrm{H}), 7.33(\mathrm{~d}, J=8.5 \mathrm{~Hz}, 1 \mathrm{H}) .{ }^{13} \mathrm{C} \mathrm{NMR}\left(\mathrm{CDCl}_{3}, 125 \mathrm{MHz}\right) \delta 165.5$ $\left(\mathrm{C}_{\mathrm{q}}\right), 148.8(\mathrm{CH}), 138.5(\mathrm{CH}), 138.1\left(\mathrm{C}_{\mathrm{q}}\right), 133.1\left(\mathrm{C}_{\mathrm{q}}\right), 122.1(\mathrm{CH})$. IR (neat): vmax $\left(\mathrm{cm}^{-1}\right)=3051(\mathrm{w}), 2125$ (s), 1572 (s), 1452 (s), 1115 (s), 1014 (s), 842 (s), 496 (s), 401 (s). HRMS (ESI): m/z calculated for $\mathrm{C}_{6} \mathrm{H}_{4} \mathrm{ClN}_{2} 139.0058[\mathrm{M}+\mathrm{H}]^{+}$, found 139.0064.

\section{5-Bromo-2-isocyanopyridine (6h).}

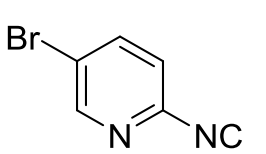

Following general procedure II with $N$-(5-bromopyridin-2-yl)formamide (S1h, 2.01 $\mathrm{g}, 10 \mathrm{mmol}, 1.0$ equiv) the isocyanide was obtained as a yellow brown powder $(1.15 \mathrm{~g}$, $6.3 \mathrm{mmol}, 63 \%)$. Degradation point $105{ }^{\circ} \mathrm{C} .{ }^{1} \mathrm{H} \mathrm{NMR}\left(\mathrm{CDCl}_{3}, 500 \mathrm{MHz}\right) \delta 8.56(\mathrm{~s}$, $1 \mathrm{H}), 7.96(\mathrm{~d}, J=8.4 \mathrm{~Hz}, 1 \mathrm{H}), 7.28(\mathrm{~d}, J=8.2 \mathrm{~Hz}, 1 \mathrm{H}) .{ }^{13} \mathrm{C} \mathrm{NMR}\left(\mathrm{CDCl}_{3}, 125 \mathrm{MHz}\right) \delta$ $165.6\left(\mathrm{C}_{\mathrm{q}}\right), 150.9(\mathrm{CH}), 141.3(\mathrm{CH}), 138.6\left(\mathrm{C}_{\mathrm{q}}\right), 122.4(\mathrm{CH}), 121.5\left(\mathrm{C}_{\mathrm{q}}\right) . \mathrm{IR}$ (neat): $v \max \left(\mathrm{cm}^{-1}\right)=3049$ (w), 2125 (s), 1566 (s), 1456 (s), 1450 (s), 1365 (s), 1094 (s), 1011 (s), 841 (s), 494 (s). HRMS (ESI): m/z calculated for $\mathrm{C}_{6} \mathrm{H}_{4} \mathrm{BrN}_{2}[\mathrm{M}+\mathrm{H}]^{+}$182.9552, found 182.9544 .

\section{2-Isocyano-5-(trifluoromethyl)pyridine (6i).}

$\mathrm{F}_{3} \mathrm{C}$ Following general procedure II with $\mathrm{N}$-(5-(trifluoromethyl)pyridin-2-yl)formamide (S1i, $1.33 \mathrm{~g}, 7.0 \mathrm{mmol}, 1.0$ equiv) the isocyanide was obtained as a dark brown powder (514 mg, $2.99 \mathrm{mmol}, 43 \%)$. The product readily degrades at room temperature. Therefore, many impurities are visible in the ${ }^{1} \mathrm{H}-\mathrm{NMR} .{ }^{1} \mathrm{H} \mathrm{NMR}\left(\mathrm{CDCl}_{3}, 500 \mathrm{MHz}\right) \delta 8.82$ (s, 1H), $8.11(\mathrm{~d}, J=8.1 \mathrm{~Hz}, 1 \mathrm{H}), 7.53(\mathrm{~d}, J=8.1 \mathrm{~Hz}, 1 \mathrm{H})$.

\section{2-Isocyano-6-methylpyridine (6j).}

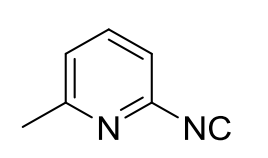

Following general procedure II with $N$-(6-methylpyridin-2-yl)formamide (S1j, $1.36 \mathrm{~g}$, 10 mmol, 1.0 equiv) 2-isocyano-4-methylpyridine was obtained as a dark brown powder (709 mg, $6.0 \mathrm{mmol}, 60 \%)$. Degradation point: $49-51{ }^{\circ} \mathrm{C} .{ }^{1} \mathrm{H}$ NMR $\left(\mathrm{CDCl}_{3}, 500 \mathrm{MHz}\right) \delta$ $7.68(\mathrm{t}, J=7.8 \mathrm{~Hz}, 1 \mathrm{H}), 7.22(\mathrm{~d}, J=7.8 \mathrm{~Hz}, 1 \mathrm{H}), 7.16(\mathrm{~d}, J=7.8 \mathrm{~Hz}, 1 \mathrm{H}) 2.55(\mathrm{~s}, 3 \mathrm{H}) .{ }^{13} \mathrm{C} \mathrm{NMR}\left(\mathrm{CDCl}_{3}\right.$, $125 \mathrm{MHz}) \delta 163.1\left(\mathrm{C}_{\mathrm{q}}\right), 159.6\left(\mathrm{C}_{\mathrm{q}}\right), 139.6\left(\mathrm{C}_{\mathrm{q}}\right), 138.7(\mathrm{CH}), 124.4(\mathrm{CH}), 118.3(\mathrm{CH}), 18.3\left(\mathrm{CH}_{3}\right) . \mathrm{IR}$ (neat): vmax $\left(\mathrm{cm}^{-1}\right)=3078(\mathrm{w}), 2130(\mathrm{~s}), 1595(\mathrm{~s}), 1450(\mathrm{~s}), 796(\mathrm{~s}), 633(\mathrm{~s}), 538(\mathrm{~s}), 496(\mathrm{~s}), 401(\mathrm{~s})$. HRMS (ESI): $m / z$ calculated for $\mathrm{C}_{7} \mathrm{H}_{7} \mathrm{~N}_{2} 119.0604[\mathrm{M}+\mathrm{H}]^{+}$, found 119.0609 . 
6-Chloro-2-isocyanopyridine (6k).

Following general procedure II with $\mathrm{N}$-(6-chloropyridin-2-yl)formamide (S1k, 1.57 g, $10 \mathrm{mmol}, 1.0$ equiv) 6-bromo-2-isocyanopyridine was obtained as a green powder (804 mg, $5.8 \mathrm{mmol}, 58 \%)$. Degradation point: $68{ }^{\circ} \mathrm{C} .{ }^{1} \mathrm{H} \mathrm{NMR}\left(\mathrm{CDCl}_{3}, 500 \mathrm{MHz}\right) \delta$ $7.78(\mathrm{t}, J=8.0 \mathrm{~Hz}, 1 \mathrm{H}), 7.43(\mathrm{~d}, J=8.0 \mathrm{~Hz}, 1 \mathrm{H}), 7.30(\mathrm{~d}, J=8.0 \mathrm{~Hz}, 1 \mathrm{H}) .{ }^{13} \mathrm{C} \mathrm{NMR}\left(\mathrm{CDCl}_{3}, 125 \mathrm{MHz}\right) \delta$ $165.9\left(\mathrm{C}_{\mathrm{q}}\right), 151.1\left(\mathrm{C}_{\mathrm{q}}\right), 141.0(\mathrm{CH}), 139.2\left(\mathrm{C}_{\mathrm{q}}\right), 125.8(\mathrm{CH}), 119.8(\mathrm{CH})$. IR (neat): vmax $\left(\mathrm{cm}^{-1}\right)=3082$ (w), 2124 (s), 1574 (s), 1427 (s), 1163 (s), 989 (s), 864 (s), 802 (s), 532 (s), 498 (s), 413 (s), 401 (s). HRMS (ESI): $m / z$ calculated for $\mathrm{C}_{6} \mathrm{H}_{4} \mathrm{ClN}_{2} 139.0058[\mathrm{M}+\mathrm{H}]^{+}$, found 139.0063 .

\section{6-Bromo-2-isocyanopyridine (61).}

Following general procedure II with $N$-(6-bromopyridin-2-yl)formamide (13, $2.01 \mathrm{~g}$, $10 \mathrm{mmol}, 1.0$ equiv) 6-bromo-2-isocyanopyridine was obtained as a green powder $\mathrm{Br}(1.30 \mathrm{~g}, 7.1 \mathrm{mmol}, 71 \%)$. Degradation point: $88{ }^{\circ} \mathrm{C} .{ }^{1} \mathrm{H}$ NMR $\left(\mathrm{CDCl}_{3}, 500 \mathrm{MHz}\right) \delta$
$7.69(\mathrm{t}, J=8.0 \mathrm{~Hz}, 1 \mathrm{H}), 7.59(\mathrm{~d}, J=8.0 \mathrm{~Hz}, 1 \mathrm{H}), 7.33(\mathrm{~d}, J=8.0 \mathrm{~Hz}, 1 \mathrm{H}) .{ }^{13} \mathrm{C}$ NMR $\left(\mathrm{CDCl}_{3}, 125 \mathrm{MHz}\right) \delta$ $166.1\left(\mathrm{C}_{\mathrm{q}}\right), 141.2(\mathrm{CH}), 140.6\left(\mathrm{C}_{\mathrm{q}}\right), 139.3\left(\mathrm{C}_{\mathrm{q}}\right) 129.6(\mathrm{CH}), 120.1(\mathrm{CH})$. IR (neat): $v \max \left(\mathrm{cm}^{-1}\right)=3056$ (w), 2131 (s), 1570 (s), 1431 (s), 989 (s), 802 (s), 631 (s), 538 (s), 503 (s), 496 (s), 436 (s), 405 (s). HRMS (ESI): $m / z$ calculated for $\mathrm{C}_{6} \mathrm{H}_{4} \mathrm{BrN}_{2}[\mathrm{M}+\mathrm{H}]^{+} 182.9552$, found 182.9546 .

\section{General procedure III: Synthesis of 2-(N-benzylacetamido)-N-(pyridin-2-yl)-4- methylpentan-amides (7).}

Unless stated otherwise: Benzylamine ( $109 \mu \mathrm{L}, 1.0 \mathrm{mmol}, 1.0$ equiv) and the 3-methylbutyraldehyde (108 $\mu \mathrm{L}, 1.0 \mathrm{mmol}, 1.0$ equiv) were dissolved in $\mathrm{MeOH}(3 \mathrm{~mL})$ and prestirred for 2 hours at room temperature. Acetic acid ( $86 \mu \mathrm{L}, 1.5 \mathrm{mmol}, 1.5$ equiv) and a 2-isocyanopyridine (6,1.2 mmol, 1.2 equiv) were added subsequently. Additional $\mathrm{MeOH}(1 \mathrm{~mL})$ was added and the reaction mixture stirred for 48 hours at room temperature. The solvent was removed under reduced pressure and the crude mixture was purified by flash chromatography (cyclohexane : EtOAc) to yield the Ugi products $\mathbf{7}$ as foamy solids.

\section{2-( $N$-benzylacetamido)-4-methyl- $N$-(3-methylpyridin-2-yl)pentanamide (7a).}

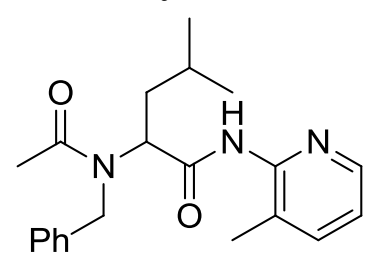

Following general procedure III with 2-isocyano-3-methylpyridine (6a, 142 $\mathrm{mg}, 1.2 \mathrm{mmol}, 1.2$ equiv) the Ugi product was obtained as a light-brown oil (233 mg, $0.66 \mathrm{mmol}, 66 \%)$. Flash chromatography: (EtOAc). ${ }^{1} \mathrm{H} \mathrm{NMR}\left(\mathrm{CDCl}_{3}\right.$, $500 \mathrm{MHz}) \delta 8.92(\mathrm{bs}, 1 \mathrm{H}), 8.30(\mathrm{~d}, J=3.9 \mathrm{~Hz}, 1 \mathrm{H}), 7.51(\mathrm{~d}, J=7.4 \mathrm{~Hz}, 1 \mathrm{H})$, 7.37-7.20 (m, 5H), $7.06(\mathrm{t}, J=6.0 \mathrm{~Hz}, 1 \mathrm{H}), 5.19(\mathrm{t}, J=7.5 \mathrm{~Hz}, 1 \mathrm{H}), 4.65(\mathrm{~s}$, 2H), $2.29(\mathrm{~s}, 3 \mathrm{H}), 2.21(\mathrm{~s}, 3 \mathrm{H}), 1.97(\mathrm{~m}, 1 \mathrm{H}), 1.58(\mathrm{~m}, 1 \mathrm{H}), 1.46(\mathrm{~m}, 1 \mathrm{H}), 0.88-0.86(\mathrm{~m}, 6 \mathrm{H}) .{ }^{13} \mathrm{C} \mathrm{NMR}$ $\left(\mathrm{CDCl}_{3}, 125 \mathrm{MHz}\right) \delta 173.6\left(\mathrm{C}_{\mathrm{q}}\right), 169.0\left(\mathrm{C}_{\mathrm{q}}\right), 149.3\left(\mathrm{C}_{\mathrm{q}}\right), 146.0(\mathrm{CH}), 139.4(\mathrm{CH}), 137.1\left(\mathrm{C}_{\mathrm{q}}\right), 128.8(\mathrm{CH})$, $127.3(\mathrm{CH}), 126.8\left(\mathrm{C}_{\mathrm{q}}\right), 126.0(\mathrm{CH}), 121.2(\mathrm{CH}), 57.1(\mathrm{CH}), 49.5\left(\mathrm{CH}_{2}\right), 36.9\left(\mathrm{CH}_{2}\right), 25.2(\mathrm{CH}), 22.9$ $\left(\mathrm{CH}_{3}\right), 22.4\left(\mathrm{CH}_{3}\right), 22.2\left(\mathrm{CH}_{3}\right), 17.9\left(\mathrm{CH}_{3}\right)$. IR (neat): vmax $\left(\mathrm{cm}^{-1}\right)=2957(\mathrm{w}), 1674(\mathrm{~m}), 1448(\mathrm{~s}), 1420$ (s), 633 (s), 534 (s), 496 (s), 401 (s). HRMS (ESI): $m / z$ calculated for $\mathrm{C}_{21} \mathrm{H}_{28} \mathrm{~N}_{3} \mathrm{O}_{2}[\mathrm{M}+\mathrm{H}]^{+} 354.2176$, found 354.2193. 


\section{2-( $N$-benzylacetamido)- $N$-(3-bromopyridin-2-yl)-4-methylpentanamide (7b).}<smiles>CC(=O)N(Cc1ccccc1)C(CC(C)C)C(=O)Nc1ncccc1Br</smiles>

Following general procedure III with 3-bromo-2-isocyanopyridine (6b, 220 $\mathrm{mg}, 1.2 \mathrm{mmol}, 1.2$ equiv) the Ugi product was obtained as a dark yellow oil (172 mg, $0.41 \mathrm{mmol}, 41 \%)$. Flash chromatography: (EtOAc). ${ }^{1} \mathrm{H} \mathrm{NMR}\left(\mathrm{CDCl}_{3}\right.$, $500 \mathrm{MHz}) \delta 9.11(\mathrm{bs}, 1 \mathrm{H}), 8.43(\mathrm{~d}, J=4.1 \mathrm{~Hz}, 1 \mathrm{H}), 7.87(\mathrm{~d}, J=7.9 \mathrm{~Hz}, 1 \mathrm{H})$, $7.38-7.18(\mathrm{~m}, 5 \mathrm{H}), 6.97(\mathrm{dd}, J=7.6,4.5 \mathrm{~Hz}, 1 \mathrm{H}), 5.22(\mathrm{t}, J=6.5 \mathrm{~Hz}, 1 \mathrm{H}), 4.63$ $(\mathrm{s}, 2 \mathrm{H}), 2.14(\mathrm{~s}, 3 \mathrm{H}), 2.01(\mathrm{~m}, 1 \mathrm{H}), 1.58(\mathrm{~m}, 1 \mathrm{H}), 1.46(\mathrm{~m}, 1 \mathrm{H}), 0.92(\mathrm{~d}, J=5.0$ $\mathrm{Hz}, 3 \mathrm{H}), 0.88(\mathrm{~d}, J=5.0 \mathrm{~Hz}, 3 \mathrm{H}) .{ }^{13} \mathrm{C} \mathrm{NMR}\left(\mathrm{CDCl}_{3}, 125 \mathrm{MHz}\right) \delta 173.6\left(\mathrm{C}_{\mathrm{q}}\right), 168.7\left(\mathrm{C}_{\mathrm{q}}\right), 148.6\left(\mathrm{C}_{\mathrm{q}}\right)$, $147.3(\mathrm{CH}), 141.3(\mathrm{CH}), 137.0\left(\mathrm{C}_{\mathrm{q}}\right), 128.8(\mathrm{CH}), 127.4(\mathrm{CH}), 126.1(\mathrm{CH}), 121.2(\mathrm{CH}), 111.6\left(\mathrm{C}_{\mathrm{q}}\right), 57.3$ $(\mathrm{CH}), 49.5\left(\mathrm{CH}_{2}\right), 36.5\left(\mathrm{CH}_{2}\right), 25.2(\mathrm{CH}), 22.9\left(\mathrm{CH}_{3}\right), 22.4\left(\mathrm{CH}_{3}\right), 22.3\left(\mathrm{CH}_{3}\right)$. IR (neat): vmax $\left(\mathrm{cm}^{-1}\right)=$ 2956 (w), 1628 (m), 1497 (s), 1431 (m), 633 (s), 534 (s), 498 (s), 405 (s). HRMS (ESI): m/z calculated for $\mathrm{C}_{20} \mathrm{H}_{25} \mathrm{BrN}_{3} \mathrm{O}_{2}[\mathrm{M}+\mathrm{H}]^{+} 418.1125$, found 418.1130 .

\section{2-( $N$-benzylacetamido)-4-methyl- $N$-(4-methylpyridin-2-yl)pentanamide (7c).}<smiles>CC(=O)N(Cc1ccccc1)C(CC(C)C)C(=O)Nc1cc(C)ccn1</smiles>

Following general procedure III with 2-isocyano-4-methylpyridine (6c, 142 $\mathrm{mg}, 1.2 \mathrm{mmol}, 1.2$ equiv) the Ugi product was obtained as an off-white (233 $\mathrm{mg}, 0.66 \mathrm{mmol}, 66 \%$ ). Flash chromatography: (cyclohexane $:$ EtOAc $=2: 1$ ). m.p.: $106{ }^{\circ} \mathrm{C} .{ }^{1} \mathrm{H}$ NMR $\left(\mathrm{CDCl}_{3}, 500 \mathrm{MHz}\right) \delta 9.03(\mathrm{bs}, 1 \mathrm{H}), 8.14(\mathrm{~d}, J=4.8 \mathrm{~Hz}$, $1 \mathrm{H}), 7.82(\mathrm{~s}, 1 \mathrm{H}), 7.28-7.17(\mathrm{~m}, 5 \mathrm{H}), 6.84(\mathrm{~d}, J=4.6 \mathrm{~Hz}, 1 \mathrm{H}), 5.22(\mathrm{t}, J=7.2$ $\mathrm{Hz}, 1 \mathrm{H}), 4.66(\mathrm{~d}, J=17.5 \mathrm{~Hz}, 1 \mathrm{H}), 4.60(\mathrm{~d}, J=17.5 \mathrm{~Hz}, 1 \mathrm{H}), 2,31(\mathrm{~s}, 3 \mathrm{H}), 2.14$ $(\mathrm{s}, 3 \mathrm{H}), 1.95(\mathrm{~m}, 1 \mathrm{H}), 1.56-1.45(\mathrm{~m}, 2 \mathrm{H}), 0.88(\mathrm{~d}, J=6.5 \mathrm{~Hz}, 3 \mathrm{H}), 0.87(\mathrm{~d}, J=$ $6.5 \mathrm{~Hz}, 3 \mathrm{H}) .{ }^{13} \mathrm{C} \mathrm{NMR}\left(\mathrm{CDCl}_{3}, 125 \mathrm{MHz}\right) \delta 173.2\left(\mathrm{C}_{\mathrm{q}}\right), 169.5\left(\mathrm{C}_{\mathrm{q}}\right), 151.2\left(\mathrm{C}_{\mathrm{q}}\right), 149.5\left(\mathrm{C}_{\mathrm{q}}\right), 147.6(\mathrm{CH})$, $137.0\left(\mathrm{C}_{\mathrm{q}}\right), 128.8(\mathrm{CH}), 127.3(\mathrm{CH}), 126.0(\mathrm{CH}), 120.9(\mathrm{CH}), 114.6(\mathrm{CH}), 56.8(\mathrm{CH}), 49.2\left(\mathrm{CH}_{2}\right), 36.8$ $\left(\mathrm{CH}_{2}\right), 25.2(\mathrm{CH}), 22.8\left(\mathrm{CH}_{3}\right), 22.3\left(\mathrm{CH}_{3}\right), 22.3\left(\mathrm{CH}_{3}\right), 21.3\left(\mathrm{CH}_{3}\right)$. IR (neat): vmax $\left(\mathrm{cm}^{-1}\right)=2957(\mathrm{w})$, 1697 (s), 1630 (m), 1568 (s), 1414 (s), 633 (s), 523 (s), 498 (s), 407 (s). HRMS (ESI): m/z calculated for $\mathrm{C}_{21} \mathrm{H}_{28} \mathrm{~N}_{3} \mathrm{O}_{2}[\mathrm{M}+\mathrm{H}]^{+}$354.2176, found 354.2185.

\section{2-( $N$-benzylacetamido)- $N$-(4-bromopyridin-2-yl)-4-methylpentanamide (7d).}<smiles>CC(=O)N(Cc1ccccc1)C(CC(C)C)C(=O)Nc1cc(Br)ccn1</smiles>

Following general procedure III with 4-bromo-2-isocyanopyridine (6d, 220 $\mathrm{mg}, 1.2 \mathrm{mmol}, 1.2$ equiv) the Ugi product was obtained as a yellow solid (272 $\mathrm{mg}, 0.65 \mathrm{mmol}, 65 \%$ ). Flash chromatography: (cyclohexane : EtOAc = 1:1). m.p.: $111^{\circ} \mathrm{C} .{ }^{1} \mathrm{H}$ NMR $\left(\mathrm{CDCl}_{3}, 500 \mathrm{MHz}\right) \delta 9.21(\mathrm{bs}, 1 \mathrm{H}), 8.19(\mathrm{~s}, 1 \mathrm{H}), 8.10(\mathrm{~d}$, $J=5.0 \mathrm{~Hz}, 1 \mathrm{H}), 7.27-7.22(\mathrm{~m}, 5 \mathrm{H}), 7.17(\mathrm{~d}, J=6.6 \mathrm{~Hz}, 1 \mathrm{H}), 5.17(\mathrm{t}, J=6.8$ $\mathrm{Hz}, 1 \mathrm{H}), 4.66(\mathrm{~d}, J=17.5 \mathrm{~Hz}, 1 \mathrm{H}), 4.57(\mathrm{~d}, J=17.5 \mathrm{~Hz}, 1 \mathrm{H}), 2.18(\mathrm{~s}, 3 \mathrm{H}), 1.94$ $(\mathrm{m}, 1 \mathrm{H}), 1.52(\mathrm{~m}, 2 \mathrm{H}), 0.88(\mathrm{~m}, 6 \mathrm{H}) .{ }^{13} \mathrm{C} \mathrm{NMR}\left(\mathrm{CDCl}_{3}, 125 \mathrm{MHz}\right) \delta 173.4\left(\mathrm{C}_{\mathrm{q}}\right)$,

$169.6\left(\mathrm{C}_{\mathrm{q}}\right), 151.9\left(\mathrm{C}_{\mathrm{q}}\right), 148.5(\mathrm{CH}), 136.6\left(\mathrm{C}_{\mathrm{q}}\right), 134.1\left(\mathrm{C}_{\mathrm{q}}\right), 128.8(\mathrm{CH}), 127.5(\mathrm{CH}), 126.2(\mathrm{CH}), 123.0$ $(\mathrm{CH}), 117.1(\mathrm{CH}), 56.8(\mathrm{CH}), 49.4\left(\mathrm{CH}_{2}\right), 36.6\left(\mathrm{CH}_{2}\right), 25.1\left(\mathrm{CH}_{3}\right), 22.8\left(\mathrm{CH}_{3}\right), 22.4\left(\mathrm{CH}_{3}\right), 22.3\left(\mathrm{CH}_{3}\right)$. IR (neat): vmax $\left(\mathrm{cm}^{-1}\right)=2957(\mathrm{w}), 1628(\mathrm{~s}), 1560(\mathrm{~s}), 1396(\mathrm{~s}), 633$ (s), 536 (s), 498 (s), 401 (s). HRMS (ESI): $m / z$ calculated for $\mathrm{C}_{20} \mathrm{H}_{25} \mathrm{BrN}_{3} \mathrm{O}_{2}[\mathrm{M}+\mathrm{H}]^{+} 418.1125$, found 418.1128 . 


\section{2-( $N$-benzylacetamido)-4-methyl- $N$-(5-methylpyridin-2-yl)pentanamide (7e).}<smiles>CC(=O)N(Cc1ccccc1)C(CC(C)C)C(=O)Nc1ccc(C)cn1</smiles>

Following general procedure III with 2-isocyano-5-methylpyridine (6e, 142 $\mathrm{mg}, 1.2 \mathrm{mmol}, 1.2$ equiv) the Ugi product was obtained as a yellow solid (205 $\mathrm{mg}, 0.58 \mathrm{mmol}, 58 \%$ ). Flash chromatography: (cyclohexane : EtOAc $=2: 1$ ). m.p.: $90{ }^{\circ} \mathrm{C} .{ }^{1} \mathrm{H}$ NMR $\left(\mathrm{CDCl}_{3}, 500 \mathrm{MHz}\right) \delta 8.98(\mathrm{bs}, 1 \mathrm{H}), 8.10(\mathrm{~s}, 1 \mathrm{H}), 7.85(\mathrm{~d}$, $J=8.4 \mathrm{~Hz}, 1 \mathrm{H}), 7.44(\mathrm{~d}, J=8.4 \mathrm{~Hz}, 1 \mathrm{H}), 7.26-7.16(\mathrm{~m}, 5 \mathrm{H}), 5.22(\mathrm{~m}, 1 \mathrm{H})$, $4.65(\mathrm{~d}, J=17.5 \mathrm{~Hz}, 1 \mathrm{H}), 4.59(\mathrm{~d}, J=17.5 \mathrm{~Hz}, 1 \mathrm{H}), 2.27(\mathrm{~s}, 3 \mathrm{H}), 2.13(\mathrm{~s}, 3 \mathrm{H})$, $1.92(\mathrm{~m}, 1 \mathrm{H}), 1.55-1.48(\mathrm{~m}, 2 \mathrm{H}), 0.88(\mathrm{~d}, J=6.5 \mathrm{~Hz}, 3 \mathrm{H}), 0.86(\mathrm{~d}, J=6.7 \mathrm{~Hz}, 3 \mathrm{H}) .{ }^{13} \mathrm{C} \mathrm{NMR}\left(\mathrm{CDCl}_{3}\right.$, $125 \mathrm{MHz}) \delta 173.2\left(\mathrm{C}_{\mathrm{q}}\right), 169.2\left(\mathrm{C}_{\mathrm{q}}\right) 149.0\left(\mathrm{C}_{\mathrm{q}}\right), 147.8(\mathrm{CH}), 138.5(\mathrm{CH}), 137.0\left(\mathrm{C}_{\mathrm{q}}\right), 129.0\left(\mathrm{C}_{\mathrm{q}}\right), 128.7$ $(\mathrm{CH}), 127.3(\mathrm{CH}), 126.0(\mathrm{CH}), 113.5(\mathrm{CH}), 56.7(\mathrm{CH}), 49.2\left(\mathrm{CH}_{2}\right), 36.8\left(\mathrm{CH}_{2}\right), 25.1(\mathrm{CH}), 22.7\left(\mathrm{CH}_{3}\right)$, $22.4\left(\mathrm{CH}_{3}\right), 22.4\left(\mathrm{CH}_{3}\right), 17.8\left(\mathrm{CH}_{3}\right)$. IR (neat): vmax $\left(\mathrm{cm}^{-1}\right)=2957(\mathrm{w}), 1630(\mathrm{~m}), 1522(\mathrm{~s}), 1385(\mathrm{~s})$, 1298 (s), 633 (s), $534(\mathrm{~m}), 498$ (s). HRMS (ESI): $m / z$ calculated for $\mathrm{C}_{21} \mathrm{H}_{28} \mathrm{~N}_{3} \mathrm{O}_{2}[\mathrm{M}+\mathrm{H}]^{+} 354.2176$, found 354.2193 .

\section{2-( $N$-benzylacetamido)- $N$-(5-fluoropyridin-2-yl)-4-methylpentanamide (7f).}

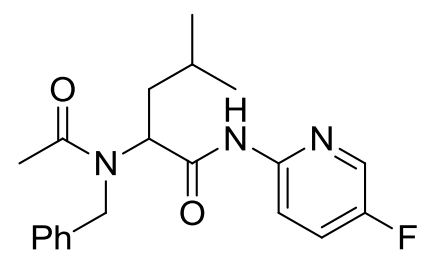

Following general procedure III with 5-fluoro-2-isocyanopyridine (6f, 147 $\mathrm{mg}, 1.2 \mathrm{mmol}, 1.2$ equiv) the Ugi product was obtained as an off-white solid $(207 \mathrm{mg}, 0.58 \mathrm{mmol}, 58 \%)$. Flash chromatography: (cyclohexane : EtOAc $=$ 3:1). m.p.: $112{ }^{\circ} \mathrm{C} .{ }^{1} \mathrm{H}$ NMR $\left(\mathrm{CDCl}_{3}, 500 \mathrm{MHz}\right) \delta 9.17$ (bs, $\left.1 \mathrm{H}\right), 8.16(\mathrm{~s}$, $1 \mathrm{H}), 7.98(\mathrm{dd}, J=9.1,3.8 \mathrm{~Hz}, 1 \mathrm{H}), 7.37(\mathrm{~m}, 1 \mathrm{H}), 7.28-7.17(\mathrm{~m}, 5 \mathrm{H}), 5.21$ $(\mathrm{m}, 1 \mathrm{H}), 4.67(\mathrm{~d}, J=17.5 \mathrm{~Hz}, 1 \mathrm{H}), 4.60 \mathrm{~J}=17.5 \mathrm{~Hz}, 1 \mathrm{H}), 2.20(3 \mathrm{H}), 1.96$ $(\mathrm{m}, 1 \mathrm{H}), 1.56-1.53(\mathrm{~m}, 2 \mathrm{H}), 0.91(\mathrm{~m}, 6 \mathrm{H}) .{ }^{13} \mathrm{C} \mathrm{NMR}\left(\mathrm{CDCl}_{3}, 125 \mathrm{MHz}\right) \delta 173.4\left(\mathrm{C}_{\mathrm{q}}\right), 169.1\left(\mathrm{C}_{\mathrm{q}}\right) 156.2(\mathrm{~d}$, $\left.J=250 \mathrm{~Hz}, \mathrm{C}_{\mathrm{q}}\right), 147.4\left(\mathrm{C}_{\mathrm{q}}\right), 136.7\left(\mathrm{C}_{\mathrm{q}}\right) 135.5(\mathrm{~d}, J=25 \mathrm{~Hz}, \mathrm{CH}), 128.8(\mathrm{CH}), 127.5(\mathrm{CH}), 126.1(\mathrm{CH})$, $124.8(\mathrm{~d}, \mathrm{~J}=20 \mathrm{~Hz}, \mathrm{CH}), 114.6(\mathrm{CH}), 56.7(\mathrm{CH}), 49.3\left(\mathrm{CH}_{2}\right), 36.6\left(\mathrm{CH}_{2}\right), 25.1(\mathrm{CH}), 22.7\left(\mathrm{CH}_{3}\right), 22.4$ $\left(\mathrm{CH}_{3}\right), 22.4\left(\mathrm{CH}_{3}\right)$. IR (neat): vmax $\left(\mathrm{cm}^{-1}\right)=2959(\mathrm{w}), 1630(\mathrm{~s}), 1526(\mathrm{~s}), 1472(\mathrm{~s}), 1391(\mathrm{~s}), 633(\mathrm{~s}), 534$ (s), 498 (s). HRMS (ESI): $m / z$ calculated for $\mathrm{C}_{20} \mathrm{H}_{25} \mathrm{FN}_{3} \mathrm{O}_{2}[\mathrm{M}+\mathrm{H}]^{+} 358.1925$, found 358.1932.

\section{2-( $N$-benzylacetamido)- $N$-(5-chloropyridin-2-yl)-4-methylpentanamide (7g).}<smiles>CC(=O)N(Cc1ccccc1)C(CC(C)C)C(=O)Nc1ccc(Cl)cn1</smiles>

Following general procedure III with 5-chloro-2-isocyanopyridine (6g, $166 \mathrm{mg}, 1.2 \mathrm{mmol}, 1.2$ equiv) the Ugi product was obtained as an orange solid (232 mg, $0.62 \mathrm{mmol}, 62 \%$ ). Flash chromatography: (cyclohexane : EtOAc $=4: 1)$. m.p.: $112{ }^{\circ} \mathrm{C} .{ }^{1} \mathrm{H}$ NMR $\left(\mathrm{CDCl}_{3}, 500 \mathrm{MHz}\right) \delta 9.16(\mathrm{bs}, 1 \mathrm{H})$, $8.24(\mathrm{~s}, 1 \mathrm{H}), 7.92(\mathrm{~d}, J=8.9 \mathrm{~Hz}, 1 \mathrm{H}), 7.58(\mathrm{~d}, J=8.8 \mathrm{~Hz}), 7.26-7.15(\mathrm{~m}$, $5 \mathrm{H}), 5.18(\mathrm{~m}, 1 \mathrm{H}), 4.65(\mathrm{~d}, J=17.4 \mathrm{~Hz}, 1 \mathrm{H}), 4.57(\mathrm{~d}, J=17.4 \mathrm{~Hz}, 1 \mathrm{H}), 2.18$

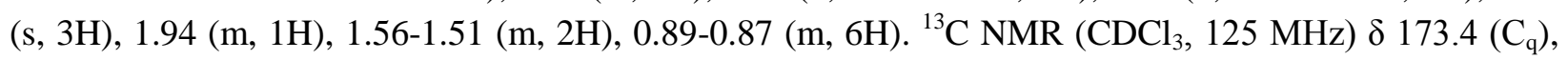
$169.4\left(\mathrm{C}_{\mathrm{q}}\right) 149.5\left(\mathrm{C}_{\mathrm{q}}\right), 146.6(\mathrm{CH}), 137.6(\mathrm{CH}) 136.6\left(\mathrm{C}_{\mathrm{q}}\right), 128.8(\mathrm{CH}), 127.5(\mathrm{CH}), 126.6\left(\mathrm{C}_{\mathrm{q}}\right), 126.1$ $(\mathrm{CH}), 114.5(\mathrm{CH}), 56.8(\mathrm{CH}), 49.4\left(\mathrm{CH}_{2}\right), 36.5\left(\mathrm{CH}_{2}\right), 25.1(\mathrm{CH}), 22.7\left(\mathrm{CH}_{3}\right), 22.4\left(\mathrm{CH}_{3}\right), 22.4\left(\mathrm{CH}_{3}\right)$. IR (neat): vmax $\left(\mathrm{cm}^{-1}\right)=2957(\mathrm{w}), 1628(\mathrm{~m}), 1522(\mathrm{~m}), 1375(\mathrm{~s}), 1292(\mathrm{~s}), 633(\mathrm{~s}), 538$ (s), 498 (s). HRMS (ESI): $m / z$ calculated for $\mathrm{C}_{20} \mathrm{H}_{25} \mathrm{ClN}_{3} \mathrm{O}_{2}[\mathrm{M}+\mathrm{H}]^{+} 374.1630$, found 374.1633 . 


\section{2-( $N$-benzylacetamido)- $N$-(5-bromopyridin-2-yl)-4-methylpentanamide (7h).}<smiles>CC(=O)N(Cc1ccccc1)C(CC(C)C)C(=O)Nc1ccc(Br)cn1</smiles>

Following general procedure III with 5-bromo-2-isocyanopyridine (6h, $220 \mathrm{mg}, 1.2 \mathrm{mmol}, 1.2$ equiv) the Ugi product was obtained as a brown solid (318 mg, $0.76 \mathrm{mmol}, 76 \%$ ). Flash chromatography: (cyclohexane : EtOAc $=3: 1)$. m.p.: $110{ }^{\circ} \mathrm{C} .{ }^{1} \mathrm{H}$ NMR $\left(\mathrm{CDCl}_{3}, 500 \mathrm{MHz}\right) \delta 9.20(\mathrm{bs}, 1 \mathrm{H})$, $8.34(\mathrm{~s}, 1 \mathrm{H}), 7.89(\mathrm{~d}, J=8.8 \mathrm{~Hz}, 1 \mathrm{H}), 7.73(\mathrm{~d}, J=8.7 \mathrm{~Hz}, 1 \mathrm{H}), 7.27-7.17$ $(\mathrm{m}, 5 \mathrm{H}), 5.20(\mathrm{t}, J=6.7 \mathrm{~Hz}, 1 \mathrm{H}), 4.67(\mathrm{~d}, J=17.4 \mathrm{~Hz}, 1 \mathrm{H}), 4.59(\mathrm{~d}, J=$ $17.4 \mathrm{~Hz}, 1 \mathrm{H}), 2.20(\mathrm{~s}, 3 \mathrm{H}), 1.95(\mathrm{~m}, 1 \mathrm{H}), 1.57-1.54(\mathrm{~m}, 2 \mathrm{H}), 0.91-0.89(\mathrm{~m}, 6 \mathrm{H}) .{ }^{13} \mathrm{C} \mathrm{NMR}\left(\mathrm{CDCl}_{3}, 125\right.$ MHz) $\delta 173.4\left(\mathrm{C}_{\mathrm{q}}\right), 169.4\left(\mathrm{C}_{\mathrm{q}}\right), 149.9\left(\mathrm{C}_{\mathrm{q}}\right), 148.9(\mathrm{CH}), 140.4(\mathrm{CH}), 136.6\left(\mathrm{C}_{\mathrm{q}}\right), 128.8(\mathrm{CH}) 127.5(\mathrm{CH})$, $126.1(\mathrm{CH}), 115.1(\mathrm{CH}), 114.5\left(\mathrm{C}_{\mathrm{q}}\right), 56.8(\mathrm{CH}), 49.4\left(\mathrm{CH}_{2}\right), 36.5\left(\mathrm{CH}_{2}\right), 25.1(\mathrm{CH}), 22.7\left(\mathrm{CH}_{3}\right), 22.4$ $\left(\mathrm{CH}_{3}\right), 22.4\left(\mathrm{CH}_{3}\right)$. IR (neat): vmax $\left(\mathrm{cm}^{-1}\right)=2957(\mathrm{w}), 1626(\mathrm{~m}), 1518(\mathrm{~m}), 1367(\mathrm{~s}), 1290(\mathrm{~s}), 633(\mathrm{~s})$, 523 (m), 498 (s), 413 (s), 403 (s). HRMS (ESI): $\mathrm{m} / \mathrm{z}$ calculated for $\mathrm{C}_{20} \mathrm{H}_{25} \mathrm{BrN}_{3} \mathrm{O}_{2}[\mathrm{M}+\mathrm{H}]^{+} 418.1125$, found 418.1138 .

\section{2-( $N$-benzylacetamido)-4-methyl- $N$-(5-(trifluoromethyl)pyridin-2-yl)pentanamide (7i).}<smiles>CC(=O)N(Cc1ccccc1)C(CC(C)C)C(=O)Nc1ccc(C(F)(F)F)cn1</smiles>

Following general procedure III with 2-isocyano-5(trifluoromethyl)pyridine (6i, $207 \mathrm{mg}, 1.2 \mathrm{mmol}, 1.2$ equiv) the Ugi product was obtained as a solid (61 $\mathrm{mg}, 0.15 \mathrm{mmol}, 15 \%)$. Flash chromatography: (cyclohexane : EtOAc $=4: 1$ ). m.p.: 93-94 ${ }^{\circ} \mathrm{C} .{ }^{1} \mathrm{H}$ NMR $\left(\mathrm{CDCl}_{3}, 500 \mathrm{MHz}\right) \delta 9.47(\mathrm{bs}, 1 \mathrm{H}), 8.56(\mathrm{~s}, 1 \mathrm{H}), 8.08(\mathrm{~d}, J=8.7 \mathrm{~Hz}, 1 \mathrm{H})$, $7.85(\mathrm{~d}, J=8.7 \mathrm{~Hz}, 1 \mathrm{H}), 7.28-7.17(\mathrm{~m}, 5 \mathrm{H}), 5.21(\mathrm{~m}, 1 \mathrm{H}), 4.68(\mathrm{~d}, J=$ $17.4 \mathrm{~Hz}, 1 \mathrm{H}), 4.59$ (d, $J=17.4 \mathrm{~Hz}, 1 \mathrm{H}), 2.23(\mathrm{~s}, 3 \mathrm{H}), 1.97(\mathrm{~m}, 1 \mathrm{H}), 1.60-1.55(\mathrm{~m}, 2 \mathrm{H}), 0.92(\mathrm{~m}, 6 \mathrm{H}) .{ }^{13} \mathrm{C}$ $\operatorname{NMR}\left(\mathrm{CDCl}_{3}, 125 \mathrm{MHz}\right) \delta 173.5\left(\mathrm{C}_{\mathrm{q}}\right), 169.8\left(\mathrm{C}_{\mathrm{q}}\right) 153.8\left(\mathrm{C}_{\mathrm{q}}\right), 145.4(\mathrm{q}, J=3.7 \mathrm{~Hz}, \mathrm{CH}), 136.5\left(\mathrm{C}_{\mathrm{q}}\right), 135.3$ $(\mathrm{q}, J=3.7 \mathrm{~Hz}, \mathrm{CH}), 128.8(\mathrm{CH}), 127.6(\mathrm{CH}), 126.2(\mathrm{CH}), 123.5\left(\mathrm{q}, J=270 \mathrm{~Hz}, \mathrm{C}_{\mathrm{q}}\right), 122.3(\mathrm{q}, J=31 \mathrm{~Hz}$, $\left.\mathrm{C}_{\mathrm{q}}\right), 113.2(\mathrm{CH}), 56.9(\mathrm{CH}), 49.5\left(\mathrm{CH}_{2}\right), 36.5\left(\mathrm{CH}_{2}\right), 25.1(\mathrm{CH}), 22.7\left(\mathrm{CH}_{3}\right), 22.4\left(\mathrm{CH}_{3}\right), 22.4\left(\mathrm{CH}_{3}\right) . \mathrm{IR}$ (neat): $v \max \left(\mathrm{cm}^{-1}\right)=2957(\mathrm{w}), 1626(\mathrm{~m}), 1520(\mathrm{~s}), 1325$ (s), 908 (s), 731 (s), 633 (s), 532 (s), 496 (s). HRMS (ESI): $m / z$ calculated for $\mathrm{C}_{21} \mathrm{H}_{25} \mathrm{~F}_{3} \mathrm{~N}_{3} \mathrm{O}_{2}[\mathrm{M}+\mathrm{H}]^{+} 408.1893$, found 408.1909.

\section{2-( $N$-benzylacetamido)-4-methyl- $N$-(6-methylpyridin-2-yl)pentanamide (7j).}

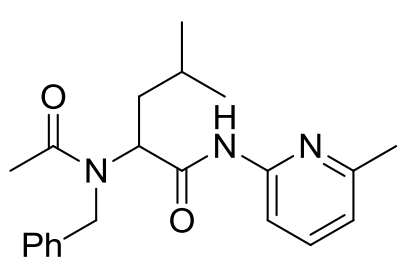
Following general procedure III with 2-isocyano-6-methylpyridine (6j, 142 $\mathrm{mg}, 1.2 \mathrm{mmol}, 1.2$ equiv) the Ugi product was obtained as an off-white solid $(219 \mathrm{mg}, 0.62 \mathrm{mmol}, 62 \%)$. Flash chromatography: (cyclohexane : EtOAc $=$ 2:1). m.p.: $84{ }^{\circ} \mathrm{C} .{ }^{1} \mathrm{H}$ NMR $\left(\mathrm{CDCl}_{3}, 500 \mathrm{MHz}\right) \delta 8.84(\mathrm{bs}, 1 \mathrm{H}), 7.79(\mathrm{~d}, J=$ $8.2 \mathrm{~Hz}, 1 \mathrm{H}), 7.53(\mathrm{t}, J=7.8 \mathrm{~Hz}, 1 \mathrm{H}), 7.30-7.18(\mathrm{~m}, 5 \mathrm{H}), 6.88(\mathrm{~d}, J=7.5 \mathrm{~Hz}$, $1 \mathrm{H}), 5.28(\mathrm{~m}, 1 \mathrm{H}), 4.68(\mathrm{~d}, J=17.6 \mathrm{~Hz}, 1 \mathrm{H}), 4.62(\mathrm{~d}, J=17.6 \mathrm{~Hz}, 1 \mathrm{H}), 2.46$ $(\mathrm{s}, 3 \mathrm{H}), 2.16(\mathrm{~s}, 3 \mathrm{H}), 1.93(\mathrm{~m}, 1 \mathrm{H}), 1.57-1.48(\mathrm{~m}, 2 \mathrm{H}), 0.91-0.81(\mathrm{~m}, 6 \mathrm{H}) \cdot{ }^{13} \mathrm{C} \mathrm{NMR}\left(\mathrm{CDCl}_{3}, 125 \mathrm{MHz}\right) \delta$ $173.1\left(\mathrm{C}_{\mathrm{q}}\right), 169.2\left(\mathrm{C}_{\mathrm{q}}\right), 157.0\left(\mathrm{C}_{\mathrm{q}}\right), 150.4\left(\mathrm{C}_{\mathrm{q}}\right), 138.3(\mathrm{CH}), 137.1\left(\mathrm{C}_{\mathrm{q}}\right), 128.8(\mathrm{CH}), 127.3(\mathrm{CH}), 126.0$ $(\mathrm{CH}), 119.2(\mathrm{CH}), 110.7(\mathrm{CH}), 56.5(\mathrm{CH}), 49.0\left(\mathrm{CH}_{2}\right), 36.8\left(\mathrm{CH}_{2}\right), 25.1(\mathrm{CH}), 24.0\left(\mathrm{CH}_{3}\right), 22.8\left(\mathrm{CH}_{3}\right)$, $22.4\left(\mathrm{CH}_{3}\right), 22.3\left(\mathrm{CH}_{3}\right)$. IR (neat): vmax $\left(\mathrm{cm}^{-1}\right)=2957(\mathrm{w}), 1630(\mathrm{~m}), 1454(\mathrm{~s}), 633(\mathrm{~s}), 536(\mathrm{~s}), 498(\mathrm{~s})$, 403 (s). HRMS (ESI): $m / z$ calculated for $\mathrm{C}_{21} \mathrm{H}_{28} \mathrm{~N}_{3} \mathrm{O}_{2}[\mathrm{M}+\mathrm{H}]^{+} 354.2176$, found 354.2182. 


\section{2-( $N$-benzylacetamido)- $N$-(6-chloropyridin-2-yl)-4-methylpentanamide (7k).}<smiles>CC(=O)N(Cc1ccccc1)C(CC(C)C)C(=O)Nc1cccc(Cl)n1</smiles>

Following general procedure III with 6-chloro-2-isocyanopyridine (6k, 166 $\mathrm{mg}, 1.2 \mathrm{mmol}, 1.2$ equiv) the Ugi product was obtained as a brown solid (243 $\mathrm{mg}, 0.65 \mathrm{mmol}, 65 \%)$. Flash chromatography: (cyclohexane : EtOAc $=7: 1$ ). m.p.: $100{ }^{\circ} \mathrm{C} .{ }^{1} \mathrm{H}$ NMR $\left(\mathrm{CDCl}_{3}, 500 \mathrm{MHz}\right) \delta 8.98$ (bs, $\left.1 \mathrm{H}\right), 7.89(\mathrm{~d}, J=8.1$ $\mathrm{Hz}, 1 \mathrm{H}), 7.58(\mathrm{t}, J=7.9 \mathrm{~Hz}, 1 \mathrm{H}), 7.30-7.18(\mathrm{~m}, 5 \mathrm{H}), 7.04(\mathrm{~d}, J=7.6 \mathrm{~Hz}, 1 \mathrm{H})$, $5.23(\mathrm{~m}, 1 \mathrm{H}), 4.67(\mathrm{~d}, J=17.4 \mathrm{~Hz}, 1 \mathrm{H}), 4.60(\mathrm{~d}, J=17.4 \mathrm{~Hz}, 1 \mathrm{H}), 2.20(\mathrm{~s}$, $3 \mathrm{H}), 1.94(\mathrm{~m}, 1 \mathrm{H}), 1.57-1.52(\mathrm{~m}, 2 \mathrm{H}), 0.91(\mathrm{~m}, 6 \mathrm{H}) .{ }^{13} \mathrm{C} \mathrm{NMR}\left(\mathrm{CDCl}_{3}, 125 \mathrm{MHz}\right) \delta 173.2\left(\mathrm{C}_{\mathrm{q}}\right), 169.5\left(\mathrm{C}_{\mathrm{q}}\right)$ $151.0\left(\mathrm{C}_{\mathrm{q}}\right), 149.0\left(\mathrm{C}_{\mathrm{q}}\right), 140.5(\mathrm{CH}), 136.7\left(\mathrm{C}_{\mathrm{q}}\right), 128.8(\mathrm{CH}), 127.5(\mathrm{CH}), 126.1(\mathrm{CH}), 119.7(\mathrm{CH}), 112.0$ $(\mathrm{CH}), 56.5(\mathrm{CH}), 49.2\left(\mathrm{CH}_{2}\right), 36.7\left(\mathrm{CH}_{2}\right), 25.1(\mathrm{CH}), 22.7\left(\mathrm{CH}_{3}\right), 22.4\left(\mathrm{CH}_{3}\right), 22.3\left(\mathrm{CH}_{3}\right)$. IR (neat): vmax $\left(\mathrm{cm}^{-1}\right)=2957(\mathrm{w}), 1628(\mathrm{~s}), 1572(\mathrm{~s}), 1435$ (s), 1157 (s), 633 (s), 536 (s), 498 (s), 424 (s), 413 (s), 403 (s). HRMS (ESI): $m / z$ calculated for $\mathrm{C}_{20} \mathrm{H}_{25} \mathrm{ClN}_{3} \mathrm{O}_{2}[\mathrm{M}+\mathrm{H}]^{+} 374.1630$, found 374.1632.

\section{2-( $N$-benzylacetamido)- $N$-(6-bromopyridin-2-yl)-4-methylpentanamide (71).}<smiles>CC(=O)N(Cc1ccccc1)C(CC(C)C)C(=O)Nc1cccc(Br)n1</smiles>

Following general procedure III with 6-bromo-2-isocyanopyridine (6l, $220 \mathrm{mg}, 1.2 \mathrm{mmol}, 1.2$ equiv) the Ugi product was obtained as a brown solid (293 mg, $0.70 \mathrm{mmol}, 70 \%)$. Flash chromatography: (cyclohexane : EtOAc $=7: 1)$. m.p.: $116{ }^{\circ} \mathrm{C} .{ }^{1} \mathrm{H} \mathrm{NMR}\left(\mathrm{CDCl}_{3}, 500 \mathrm{MHz}\right) \delta 9.06(\mathrm{bs}, 1 \mathrm{H})$, $7.89(\mathrm{~d}, J=8.2 \mathrm{~Hz}, 1 \mathrm{H}), 7.44(\mathrm{t}, J=7.8 \mathrm{~Hz}, 1 \mathrm{H}), 7.27-7.14(\mathrm{~m}, 6 \mathrm{H}), 5.27$ $(\mathrm{m}, 1 \mathrm{H}), 4.67(\mathrm{~d}, J=17.4 \mathrm{~Hz}, 1 \mathrm{H}), 4.60(\mathrm{~d}, J=17.4 \mathrm{~Hz}, 1 \mathrm{H}), 2.18(\mathrm{~s}, 3 \mathrm{H})$, $1.92(\mathrm{~m}, 1 \mathrm{H}), 1.54-1.48(\mathrm{~m}, 2 \mathrm{H}), 0.88(\mathrm{~m}, 6 \mathrm{H}) .{ }^{13} \mathrm{C} \mathrm{NMR}\left(\mathrm{CDCl}_{3}, 125 \mathrm{MHz}\right) \delta 173.1\left(\mathrm{C}_{\mathrm{q}}\right), 169.5\left(\mathrm{C}_{\mathrm{q}}\right)$ $151.2\left(\mathrm{C}_{\mathrm{q}}\right), 140.1(\mathrm{CH}), 139.3\left(\mathrm{C}_{\mathrm{q}}\right), 136.8\left(\mathrm{C}_{\mathrm{q}}\right), 128.8(\mathrm{CH}), 127.4(\mathrm{CH}), 126.1(\mathrm{CH}), 123.4(\mathrm{CH}), 112.3$ $(\mathrm{CH}), 56.4(\mathrm{CH}), 49.1\left(\mathrm{CH}_{2}\right), 36.8\left(\mathrm{CH}_{2}\right), 25.1(\mathrm{CH}), 22.7\left(\mathrm{CH}_{3}\right), 22.3\left(\mathrm{CH}_{3}\right), 22.3\left(\mathrm{CH}_{3}\right)$. IR (neat): vmax $\left(\mathrm{cm}^{-1}\right)=2957(\mathrm{w}), 1624(\mathrm{~s}), 1566(\mathrm{~s}), 1429$ (s), 1388 (s), 1155 (s), 633 (s), 530 (s), 496 (s), 403 (s). HRMS (ESI): $m / z$ calculated for $\mathrm{C}_{20} \mathrm{H}_{25} \mathrm{BrN}_{3} \mathrm{O}_{2}[\mathrm{M}+\mathrm{H}]^{+} 418.1125$, found 418.1139 .

\section{General procedure IV: Synthesis of Ugi products (9).}

An amine (1.0 mmol, 1.0 equiv) and an aldehyde (1.0 mmol, 1.0 equiv) were dissolved in $\mathrm{MeOH}$ or TFE ( $3 \mathrm{~mL}$ ) and prestirred for 2 hours at room temperature. A carboxylic acid (1.5 mmol, 1.5 equiv) and 2bromo-6-isocyanopyridine (61, $220 \mathrm{mg}, 1.2 \mathrm{mmol}, 1.2$ equiv) were added subsequently. Additional solvent $(1 \mathrm{~mL})$ was added and the reaction mixture stirred for 48 hours at room temperature. The solvent was removed under reduced pressure and the crude mixture was purified by flash chromatography (cyclohexane : EtOAc) to yield Ugi products $\mathbf{9}$ as foamy solids.

\section{$N$-benzyl- $N$-(2-((6-bromopyridin-2-yl)amino)-2-oxoethyl)acetamide (9b).}

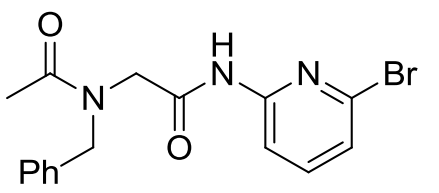

Following general procedure IV in $\mathrm{MeOH}$ with benzylamine $(109 \mu \mathrm{L}, 1.0$ mmol, 1.0 equiv), paraformaldehyde ( $45 \mathrm{mg}, 1.0 \mathrm{mmol}, 1.0$ equiv) and acetic acid ( $86 \mu \mathrm{L}, 1.5 \mathrm{mmol}, 1.5$ equiv) the Ugi product was obtained as an orange solid (140 mg, $0.39 \mathrm{mmol}, 39 \%)$. Flash chromatography: (cyclohexane : EtOAc $=$ a gradient of 10:1 to 8:1). m.p.: $191^{\circ} \mathrm{C}$. Two rotamers were present on NMR timescale $\left(\mathrm{R}^{1}: \mathrm{R}^{2}=1: 0.2\right) .{ }^{1} \mathrm{H} \mathrm{NMR}\left(\mathrm{CDCl}_{3}, 500 \mathrm{MHz}\right) \delta 8.69(\mathrm{bs}, 1 \mathrm{H}), 8.22(\mathrm{bs}, 0.2 \mathrm{H}), 8.12(\mathrm{~d}, J=8.1$ $\mathrm{Hz}, 0.2 \mathrm{H}), 8.08(\mathrm{~d}, J=7.7 \mathrm{~Hz}, 1 \mathrm{H}), 7.52(\mathrm{t}, J=8.0 \mathrm{~Hz}, 1.2 \mathrm{H}), 7.38-7-26(\mathrm{~m}, 3.6 \mathrm{H}), 7.20(\mathrm{dd}, J=4.9,2.7$ $\mathrm{Hz}, 2.4 \mathrm{H}), 4.70(\mathrm{~s}, 2.4 \mathrm{H}), 4.14(\mathrm{~s}, 2 \mathrm{H}), 4.04(\mathrm{~s}, 0.4 \mathrm{H}), 2.30(\mathrm{~s}, 3 \mathrm{H}), 2.19(\mathrm{~s}, 0.6 \mathrm{H}) .{ }^{13} \mathrm{C} \mathrm{NMR}\left(\mathrm{CDCl}_{3}, 125\right.$ $\mathrm{MHz}) \delta 172.4\left(\mathrm{C}_{\mathrm{q}}\right), 167.4\left(\mathrm{C}_{\mathrm{q}}\right), 151.0\left(\mathrm{C}_{\mathrm{q}}\right), 140.4(\mathrm{CH}), 139.3\left(\mathrm{C}_{\mathrm{q}}\right), 135.3\left(\mathrm{C}_{\mathrm{q}}\right), 129.1(\mathrm{CH}), 128.9(\mathrm{CH})$, 
$128.5(\mathrm{CH}), 128.1(\mathrm{CH}), 127.9(\mathrm{CH}), 126.6(\mathrm{CH}), 123.7(\mathrm{CH}), 112.4(\mathrm{CH}), 53.4\left(\mathrm{CH}_{2}\right), 51.6\left(\mathrm{CH}_{2}\right), 50.6$ $\left(\mathrm{CH}_{2}\right), 49.9\left(\mathrm{CH}_{2}\right), 21.8\left(\mathrm{CH}_{3}\right) 21.4\left(\mathrm{CH}_{3}\right)$. IR (neat): vmax $\left(\mathrm{cm}^{-1}\right)=3227(\mathrm{w}), 3040(\mathrm{w}), 1701(\mathrm{~s}), 1623$ (m), 1568 (s), 1431 (s), 1155 (s), 1128 (s), 789 (s), 729 (s). HRMS (ESI): m/z calculated for $\mathrm{C}_{16} \mathrm{H}_{17} \mathrm{BrN}_{3} \mathrm{O}_{2}[\mathrm{M}+\mathrm{H}]^{+}$362.0499, found 362.0485.

$N$-benzyl- $N$-(1-((6-bromopyridin-2-yl)amino)-4-methyl-1-oxopentan-2-yl)benzamide (9c).<smiles>CC(C)CC(C(=O)Nc1cccc(Br)n1)N(Cc1ccccc1)C(=O)c1ccccc1</smiles>
Following general procedure IV in TFE with benzylamine $(109 \mu \mathrm{L}, 1.0$ mmol, 1.0 equiv), 3-methylbutyraldehyde (108 $\mu \mathrm{L}, 1.0 \mathrm{mmol}, 1.0$ equiv) and benzoic acid (183 $\mathrm{mg}, 1.5 \mathrm{mmol}, 1.5$ equiv) the Ugi product was obtained as an light-brown solid (384 mg, $0.80 \mathrm{mmol}, 80 \%)$. Flash chromatography: (cyclohexane : EtOAc $=10: 1)$ m.p.: 102-103 ${ }^{\circ} \mathrm{C} .{ }^{1} \mathrm{H}$ NMR $\left(\mathrm{CDCl}_{3}, 500 \mathrm{MHz}\right) \delta 9.38(\mathrm{bs}, 1 \mathrm{H}), 7.78-6.97(\mathrm{~m}, 13 \mathrm{H}), 5.04(\mathrm{~m}$, $1 \mathrm{H}), 4.75(\mathrm{~d}, J=15.0 \mathrm{~Hz}, 1 \mathrm{H}), 4.47(\mathrm{~m}, 1 \mathrm{H}), 1.96(\mathrm{~m}, 2 \mathrm{H}), 1.72(\mathrm{~m}, 1 \mathrm{H}), 0.99(\mathrm{~m}, 6 \mathrm{H}) .{ }^{13} \mathrm{C}$ NMR $\left(\mathrm{CDCl}_{3}, 125 \mathrm{MHz}\right) \delta 174.1\left(\mathrm{C}_{\mathrm{q}}\right), 169.4\left(\mathrm{C}_{\mathrm{q}}\right), 151.1\left(\mathrm{C}_{\mathrm{q}}\right), 139.9(\mathrm{CH}), 139.2\left(\mathrm{C}_{\mathrm{q}}\right), 135.8\left(\mathrm{C}_{\mathrm{q}}\right), 135.2\left(\mathrm{C}_{\mathrm{q}}\right)$, $130.3(\mathrm{CH}), 128.6(\mathrm{CH}), 128.4(\mathrm{CH}), 127.6(\mathrm{CH}), 127.5(\mathrm{CH}), 123.2(\mathrm{CH}), 112.3(\mathrm{CH}), 57.5(\mathrm{CH}), 51.5$ $\left(\mathrm{CH}_{2}\right), 36.3\left(\mathrm{CH}_{2}\right), 24.9(\mathrm{CH}), 22.9\left(\mathrm{CH}_{3}\right), 22.0\left(\mathrm{CH}_{3}\right)$. IR (neat): vmax $\left(\mathrm{cm}^{-1}\right)=3252(\mathrm{w}), 2955(\mathrm{w}), 1697$ (s), 1564 (s), 1522 (m), 1431 (s), 1155 (s), 787 (s), 698 (m). HRMS (ESI): m/z calculated for $\mathrm{C}_{25} \mathrm{H}_{27} \mathrm{BrN}_{3} \mathrm{O}_{2}[\mathrm{M}+\mathrm{H}]^{+} 480.1281$, found 480.1272 .

\section{$N$-benzyl- $N$-(1-((6-bromopyridin-2-yl)amino)-4-methyl-1-oxopentan-2-yl)thiophene-2-carboxamide}<smiles>CC(C)CC(C(=O)Nc1cccc(Br)n1)N(Cc1ccccc1)C(=O)c1cccs1</smiles>

(9d).

Following general procedure IV in TFE with benzylamine $(109 \mu \mathrm{L}$, $1.0 \mathrm{mmol}, 1.0$ equiv), 3-methylbutyraldehyde (108 $\mu \mathrm{L}, 1.0 \mathrm{mmol}, 1.0$ equiv) and thiophene-2-carboxylic acid (192 $\mathrm{mg}, 1.5 \mathrm{mmol}, 1.5$ equiv) the Ugi product was obtained as a brown solid $(398 \mathrm{mg}, 0.82 \mathrm{mmol}$, $82 \%$ ). Flash chromatography: (cyclohexane $:$ EtOAc $=$ a gradient of

10:1 to 7:1). m.p.: $120{ }^{\circ} \mathrm{C}$. The spectra contain minor amounts of EtOAc. ${ }^{1} \mathrm{H} \mathrm{NMR}\left(\mathrm{CDCl}_{3}, 500 \mathrm{MHz}\right) \delta$ 9.18 (bs, $7.83(\mathrm{~d}, J=8.1 \mathrm{~Hz}, 1 \mathrm{H}), 7.51(\mathrm{~d}, J=2.5 \mathrm{~Hz}, 1 \mathrm{H}), 7.46-7.41(\mathrm{~m}, 2 \mathrm{H}), 7.27-7.17(\mathrm{~m}, 6 \mathrm{H}),(\mathrm{t}, J=$ $4.4 \mathrm{~Hz}, 1 \mathrm{H}), 5.04(\mathrm{~d}, J=17.1 \mathrm{~Hz}, 1 \mathrm{H}), 5.02(\mathrm{t}, J=7.3 \mathrm{~Hz}, 1 \mathrm{H}), 4.82(\mathrm{~d}, J=16.8 \mathrm{~Hz}, 1 \mathrm{H}), 1.99(\mathrm{~m}, 1 \mathrm{H})$, $1.75(\mathrm{~m}, 1 \mathrm{H}), 1.61(\mathrm{~m}, 1 \mathrm{H}), 0.90(\mathrm{~m}, 6 \mathrm{H}) \cdot{ }^{13} \mathrm{C} \mathrm{NMR}\left(\mathrm{CDCl}_{3}, 125 \mathrm{MHz}\right) \delta 169.3\left(\mathrm{C}_{\mathrm{q}}\right), 166.7\left(\mathrm{C}_{\mathrm{q}}\right), 151.2$ $\left(\mathrm{C}_{\mathrm{q}}\right), 140.1(\mathrm{CH}), 139.4\left(\mathrm{C}_{\mathrm{q}}\right), 137.1\left(\mathrm{C}_{\mathrm{q}}\right), 136.5\left(\mathrm{C}_{\mathrm{q}}\right), 130.8(\mathrm{CH}), 130.0(\mathrm{CH}), 128.7(\mathrm{CH}), 127.7(\mathrm{CH})$, $127.3(\mathrm{CH}), 127.2(\mathrm{CH}), 123.6(\mathrm{CH}), 112.5(\mathrm{CH}), 59.2(\mathrm{CH}), 51.5\left(\mathrm{CH}_{2}\right), 37.1\left(\mathrm{CH}_{2}\right), 25.2(\mathrm{CH}) .22 .6$ $\left(\mathrm{CH}_{3}\right), 22.5\left(\mathrm{CH}_{3}\right)$. IR (neat): vmax $\left(\mathrm{cm}^{-1}\right)=3240(\mathrm{w}), 2957$ (w), $1697(\mathrm{~s}), 1562$ (s), 1517 (s), $1427(\mathrm{~s})$, 1387 (s), 1155 (s), 1120 (s), 727 (s). HRMS (ESI): $m / z$ calculated for $\mathrm{C}_{23} \mathrm{H}_{25} \mathrm{BrN}_{3} \mathrm{O}_{2} \mathrm{~S}[\mathrm{M}+\mathrm{H}]^{+} 486.0845$, found 486.0831 . 
$N$-(6-bromopyridin-2-yl)-2-( $N$-(4-chlorobenzyl)pivalamido)-4-methyl-pentanamide (9e).

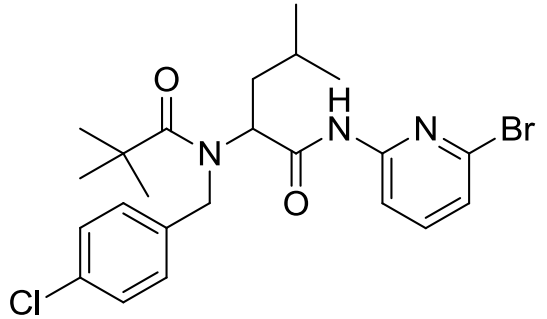

Following general procedure IV in $\mathrm{MeOH}$ with (4-chlorophenyl)methanamine (122 $\mu \mathrm{L}, 1.0 \mathrm{mmol}, 1.0$ equiv), 3-methylbutyraldehyde $(108 \mu \mathrm{L}, 1.0 \mathrm{mmol}, 1.0$ equiv) and pivalic acid (153 mg, $1.5 \mathrm{mmol}$, 1.5 equiv) the Ugi product was obtained as a yellow solid (287 $\mathrm{mg}$, $0.58 \mathrm{mmol}, 58 \%$ ). Flash chromatography: (cyclohexane : EtOAc $=\mathrm{a}$ gradient of $12: 1$ to $7: 1)$. m.p.: $117{ }^{\circ} \mathrm{C} .{ }^{1} \mathrm{H} \mathrm{NMR}\left(\mathrm{CDCl}_{3}, 500 \mathrm{MHz}\right) \delta$ $9.04(\mathrm{bs}, 1 \mathrm{H}), 7.65(\mathrm{~d}, J=8.2 \mathrm{~Hz}, 1 \mathrm{H}), 7.43(\mathrm{t}, J=8.0 \mathrm{~Hz}, 1 \mathrm{H}), 7.22-$

$7.13(\mathrm{~m}, 6 \mathrm{H}), 4.89(\mathrm{~d}, J=16.3 \mathrm{~Hz}, 1 \mathrm{H}), 4.66(\mathrm{t}, J=7.4 \mathrm{~Hz}, 1 \mathrm{H}), 4.56(\mathrm{~d}, J=16.3 \mathrm{~Hz}, 1 \mathrm{H}), 1.85(\mathrm{~m}, 1 \mathrm{H})$, $1.69(\mathrm{~m}, 1 \mathrm{H}), 1.54(\mathrm{~m}, 1 \mathrm{H}), 1.38(\mathrm{~s}, 9 \mathrm{H}), 0.91(\mathrm{~d}, J=6.6 \mathrm{~Hz}, 3 \mathrm{H}), 0.88(\mathrm{~d}, J=6.6,3 \mathrm{H}) .{ }^{13} \mathrm{C}$ NMR $\left(\mathrm{CDCl}_{3}, 125 \mathrm{MHz}\right) \delta 180.3\left(\mathrm{C}_{\mathrm{q}}\right), 169.8\left(\mathrm{C}_{\mathrm{q}}\right), 151.2\left(\mathrm{C}_{\mathrm{q}}\right), 140.0(\mathrm{CH}), 139.3\left(\mathrm{C}_{\mathrm{q}}\right), 134.7\left(\mathrm{C}_{\mathrm{q}}\right), 133.6\left(\mathrm{C}_{\mathrm{q}}\right)$, $128.9(\mathrm{CH}), 128.8(\mathrm{CH}), 123.3(\mathrm{CH}), 112.3(\mathrm{CH}), 59.1(\mathrm{CH}), 49.9\left(\mathrm{CH}_{2}\right), 40.2\left(\mathrm{C}_{\mathrm{q}}\right), 36.7\left(\mathrm{CH}_{2}\right), 28.9$ $\left(\mathrm{CH}_{3}\right), 24.9(\mathrm{CH}), 22.8\left(\mathrm{CH}_{3}\right), 22.3\left(\mathrm{CH}_{3}\right)$. IR (neat): vmax $\left(\mathrm{cm}^{-1}\right)=2957(\mathrm{w}), 1701(\mathrm{~s}), 1562(\mathrm{~s}), 1431(\mathrm{~s})$, 1155 (s), 787 (s). HRMS (ESI): $m / z$ calculated for $\mathrm{C}_{23} \mathrm{H}_{30} \mathrm{BrClN}_{3} \mathrm{O}_{2}[\mathrm{M}+\mathrm{H}]^{+}$494.1204, found 494.1201.

2-( $N$-benzylacetamido)- $N$-(6-bromopyridin-2-yl)-2-phenylacetamide (9f).

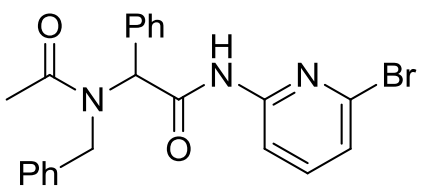

Following general procedure IV in TFE with benzylamine $(109 \mu \mathrm{L}, 1.0$ mmol, 1.0 equiv), benzaldehyde (102 $\mu \mathrm{L}, 1.0 \mathrm{mmol}, 1.0$ equiv) and acetic acid $(86 \mu \mathrm{L}, 1.5 \mathrm{mmol}, 1.5$ equiv) the Ugi product was obtained as an offwhite solid (317 mg, $0.73 \mathrm{mmol}, 73 \%$ ). Flash chromatography: (cyclohexane $:$ EtOAc $=4: 1)$. m.p.: $158-159{ }^{\circ} \mathrm{C} .{ }^{1} \mathrm{H}$ NMR $\left(\mathrm{CDCl}_{3}, 500 \mathrm{MHz}\right) \delta 8.23(\mathrm{bs}$, $1 \mathrm{H}), 8.20(\mathrm{~d}, J=8.3 \mathrm{~Hz}, 1 \mathrm{H}), 7.53(\mathrm{t}, J=8.3 \mathrm{~Hz}, 1 \mathrm{H}), 7.34(\mathrm{~m}, 2 \mathrm{H}), 7.27(\mathrm{~m}, 3 \mathrm{H}), 7.18(\mathrm{~m}, 4 \mathrm{H}), 6.98(\mathrm{~d}$, $J=7.3 \mathrm{~Hz}, 2 \mathrm{H}), 6.12(\mathrm{~s}, 1 \mathrm{H}), 4.75(\mathrm{~d}, J=17.6 \mathrm{~Hz}, 1 \mathrm{H}), 4.53(\mathrm{~d}, J=17.6,1 \mathrm{H}), 2.17(\mathrm{~s}, 3 \mathrm{H}) .{ }^{13} \mathrm{C}$ NMR $\left(\mathrm{CDCl}_{3}, 125 \mathrm{MHz}\right) \delta 172.8\left(\mathrm{C}_{\mathrm{q}}\right), 168.6\left(\mathrm{C}_{\mathrm{q}}\right), 151.1\left(\mathrm{C}_{\mathrm{q}}\right), 140.5(\mathrm{CH}), 139.2\left(\mathrm{C}_{\mathrm{q}}\right), 137.1\left(\mathrm{C}_{\mathrm{q}}\right), 133.3\left(\mathrm{C}_{\mathrm{q}}\right)$, $130.1(\mathrm{CH}), 129.2(\mathrm{CH}), 129.1(\mathrm{CH}), 128.4(\mathrm{CH}), 127.0(\mathrm{CH}), 126.0(\mathrm{CH}), 123.6(\mathrm{CH}), 112.3(\mathrm{CH}), 63.9$ $(\mathrm{CH}), 50.5\left(\mathrm{CH}_{2}\right), 22.4\left(\mathrm{CH}_{3}\right)$. IR (neat): vmax $\left(\mathrm{cm}^{-1}\right)=3238(\mathrm{w}), 3030(\mathrm{w}), 1703(\mathrm{~s}), 1634(\mathrm{~s}), 1568(\mathrm{~s})$, $1431(\mathrm{~m}), 1153$ (s), $698(\mathrm{~m}), 631$ (s), 530 (s), 500 (s). HRMS (ESI): $\mathrm{m} / z$ calculated for $\mathrm{C}_{22} \mathrm{H}_{21} \mathrm{BrN}_{3} \mathrm{O}_{2}$ [M $+\mathrm{H}]^{+}$438.0812, found 438.0804 .

\section{$N$-(2-((6-bromopyridin-2-yl)amino)-2-oxo-1-phenylethyl)- $N$-(4-methoxy-phenethyl)propionamide}<smiles>CCC(=O)N(CCP)C(C(=O)Nc1cccc(Br)n1)c1ccccc1</smiles>

$(9 \mathrm{~g})$.

Following general procedure IV in TFE with 2-(4-methoxyphenyl)ethanamine (147 $\mu \mathrm{L}, 1.0 \mathrm{mmol}, 1.0$ equiv), benzaldehyde $(102 \mu \mathrm{L}, 1.0$ mmol, 1.0 equiv) and propionic acid $(112 \mu \mathrm{L}, 1.5 \mathrm{mmol}, 1.5$ equiv) the Ugi product was obtained as an orange solid (346 $\mathrm{mg}, 0.70 \mathrm{mmol}, 70 \%)$. Flash chromatography: (cyclohexane : EtOAc $=8: 1$ ). m.p.: $152{ }^{\circ} \mathrm{C} .{ }^{1} \mathrm{H}$ $\operatorname{NMR}\left(\mathrm{CDCl}_{3}, 500 \mathrm{MHz}\right) \delta 8.23(\mathrm{bs}, 1 \mathrm{H}), 8.21(\mathrm{~d}, J=8.2 \mathrm{~Hz}, 1 \mathrm{H}), 7.52(\mathrm{t}, J=7.9 \mathrm{~Hz}, 1 \mathrm{H}), 7.50-7.41(\mathrm{~m}$, $5 \mathrm{H}), 7.17(\mathrm{~d}, J=7.7 \mathrm{~Hz}, 1 \mathrm{H}), 6.78-6.74(\mathrm{~m}, 4 \mathrm{H}), 6.19(\mathrm{~s}, 1 \mathrm{H}), 3.75(\mathrm{~s}, 3 \mathrm{H}), 3.45(\mathrm{~m}, 2 \mathrm{H}), 2.62-2.43(\mathrm{~m}$, $3 \mathrm{H}), 2.10(\mathrm{~m}, 1 \mathrm{H}), 1.20(\mathrm{t}, J=7,3 \mathrm{~Hz}, 3 \mathrm{H}) .{ }^{13} \mathrm{C} \mathrm{NMR}\left(\mathrm{CDCl}_{3}, 125 \mathrm{MHz}\right) \delta 175.0\left(\mathrm{C}_{\mathrm{q}}\right), 168.9\left(\mathrm{C}_{\mathrm{q}}\right), 158.2$ $\left(\mathrm{C}_{\mathrm{q}}\right), 151.2\left(\mathrm{C}_{\mathrm{q}}\right), 140.4(\mathrm{CH}), 139.2\left(\mathrm{C}_{\mathrm{q}}\right), 133.9\left(\mathrm{C}_{\mathrm{q}}\right), 130.2(\mathrm{CH}), 130.1\left(\mathrm{C}_{\mathrm{q}}\right), 129.4(\mathrm{CH}), 129.3(\mathrm{CH})$, $129.2(\mathrm{CH}), 123.5(\mathrm{CH}), 113.9(\mathrm{CH}), 112.3(\mathrm{CH}), 63.5(\mathrm{CH}), 55.2\left(\mathrm{CH}_{3}\right), 48.1\left(\mathrm{CH}_{2}\right), 35.4\left(\mathrm{CH}_{2}\right), 26.7$ $\left(\mathrm{CH}_{2}\right), 9.4\left(\mathrm{CH}_{3}\right)$. IR (neat): vmax $\left(\mathrm{cm}^{-1}\right)=3238(\mathrm{w}), 2937(\mathrm{w}), 1705(\mathrm{~m}), 1618(\mathrm{~s}), 1568(\mathrm{~s}), 1512(\mathrm{~s})$, 1431 (s), 1153 (s), 787 (m). HRMS (ESI): $m / z$ calculated for $\mathrm{C}_{25} \mathrm{H}_{27} \mathrm{BrN}_{3} \mathrm{O}_{3}[\mathrm{M}+\mathrm{H}]^{+}$496.1130, found 496.1123. 
$N$-(6-bromopyridin-2-yl)-2-(4-chlorophenyl)-2-(N-propylacetamido)-acetamide (9h).

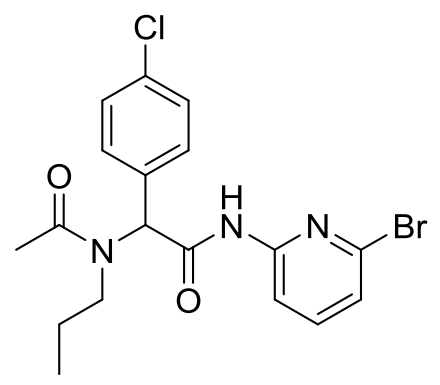

Following general procedure IV in TFE with propylamine $(82 \mu \mathrm{L}, 1.0$ mmol, 1.0 equiv), 4-chlorobenzaldehyde (141 $\mathrm{mg}, 1.0 \mathrm{mmol}, 1.0$ equiv) and acetic acid ( $86 \mu \mathrm{L}, 1.5 \mathrm{mmol}, 1.5$ equiv) the Ugi product was obtained as an off-white solid (327 mg, $0.77 \mathrm{mmol}, 77 \%$ ). Flash chromatography: (cyclohexane : EtOAc $=4: 1)$. m.p.: $166{ }^{\circ} \mathrm{C} .{ }^{1} \mathrm{H} \mathrm{NMR}\left(\mathrm{CDCl}_{3}, 500 \mathrm{MHz}\right) \delta$ $8.81(\mathrm{bs}, 1 \mathrm{H}), 8.17(\mathrm{~d}, J=8.0 \mathrm{~Hz}, 1 \mathrm{H}), 7.52(\mathrm{t}, J=8.0 \mathrm{~Hz}, 1 \mathrm{H}), 7.33$ (bs, $4 \mathrm{H}), 7.17(\mathrm{~d}, J=7.5,1 \mathrm{H}), 6.11(\mathrm{~s}, 1 \mathrm{H}), 3.25(\mathrm{t}, J=8.0 \mathrm{~Hz}, 2 \mathrm{H}), 2.25(\mathrm{~s}$, $3 \mathrm{H}), 1.45(\mathrm{~m}, 1 \mathrm{H}), 1.05(\mathrm{~m}, 1 \mathrm{H}), 0.69(\mathrm{t}, J=7.0 \mathrm{~Hz}, 3 \mathrm{H}) .{ }^{13} \mathrm{C} \mathrm{NMR}$ $\left(\mathrm{CDCl}_{3}, 125 \mathrm{MHz}\right) \delta 172.1\left(\mathrm{C}_{\mathrm{q}}\right), 168.6\left(\mathrm{C}_{\mathrm{q}}\right), 151.2\left(\mathrm{C}_{\mathrm{q}}\right), 140.4(\mathrm{CH}), 139.3$ $\left(\mathrm{C}_{\mathrm{q}}\right), 135.0\left(\mathrm{C}_{\mathrm{q}}\right), 132.8\left(\mathrm{C}_{\mathrm{q}}\right), 131.0(\mathrm{CH}), 123.6(\mathrm{CH}), 112.3(\mathrm{CH}), 62.6(\mathrm{CH}), 49.2\left(\mathrm{CH}_{2}\right), 23.0\left(\mathrm{CH}_{2}\right)$, $21.8\left(\mathrm{CH}_{3}\right) 11.1\left(\mathrm{CH}_{3}\right)$. IR (neat): vmax $\left(\mathrm{cm}^{-1}\right)=2968(\mathrm{w}), 1703(\mathrm{~m}), 1616(\mathrm{~m}), 1566(\mathrm{~s}), 1533(\mathrm{~m}), 1427$ (s), 1391 (s), 1300 (m), 1155 (s), 1128 (s), 789 (s), 731 (s), 548 (s). HRMS (ESI): m/z calculated for $\mathrm{C}_{18} \mathrm{H}_{20} \mathrm{BrClN}_{3} \mathrm{O}_{2}[\mathrm{M}+\mathrm{H}]^{+}$424.0422, found 424.0418.

\section{2-( $N$-benzylacetamido)- $N$-(6-bromopyridin-2-yl)-2-cyclohexylacetamide (9i).}

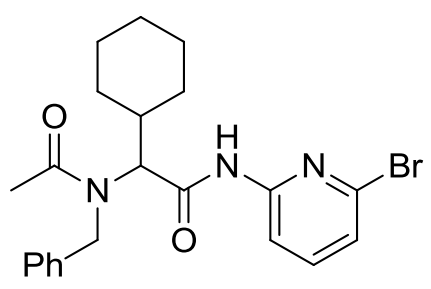

Following general procedure IV in $\mathrm{MeOH}$ with benzylamine $(109 \mu \mathrm{L}, 1.0$ mmol, 1.0 equiv), cyclohexanecarbaldehyde (121 $\mu \mathrm{L}, 1.0 \mathrm{mmol}, 1.0$ equiv) and acetic acid ( $86 \mu \mathrm{L}, 1.5 \mathrm{mmol}, 1.5$ equiv) the Ugi product was obtained as an off-white solid (396 mg, $0.89 \mathrm{mmol}, 89 \%$ ). Flash chromatography: (cyclohexane $:$ EtOAc $=$ a gradient of 10:1 to 6:1). The spectra contain minor amounts of EtOAc. m.p.: $90{ }^{\circ} \mathrm{C} .{ }^{1} \mathrm{H} \mathrm{NMR}\left(\mathrm{CDCl}_{3}, 500 \mathrm{MHz}\right) \delta 9.26$ (bs, $1 \mathrm{H}), 7.76(\mathrm{~d}, J=8.2 \mathrm{~Hz}, 1 \mathrm{H}), 7.41(\mathrm{t}, J=7.9 \mathrm{~Hz}, 1 \mathrm{H}), 7.21-7.12(\mathrm{~m}$, $6 \mathrm{H}), 4.78(\mathrm{~m}, 1 \mathrm{H}), 4.68(\mathrm{~d}, J=17.2 \mathrm{~Hz}, 1 \mathrm{H}), 4.61 \mathrm{~J}=17.2 \mathrm{~Hz}, 1 \mathrm{H}), 2.28(\mathrm{~m}, 1 \mathrm{H}), 2.17(\mathrm{~s}, 3 \mathrm{H}), 1.75-1.60$ $(\mathrm{m}, 6 \mathrm{H}), 1.20-1.14(\mathrm{~m}, 2 \mathrm{H}), 1.03-0.99(\mathrm{~m}, 2 \mathrm{H}) .{ }^{13} \mathrm{C} \mathrm{NMR}\left(\mathrm{CDCl}_{3}, 125 \mathrm{MHz}\right) \delta 173.2\left(\mathrm{C}_{\mathrm{q}}\right), 168.8\left(\mathrm{C}_{\mathrm{q}}\right)$, $151.2\left(\mathrm{C}_{\mathrm{q}}\right), 140.2(\mathrm{CH}), 139.3\left(\mathrm{C}_{\mathrm{q}}\right), 136.7\left(\mathrm{C}_{\mathrm{q}}\right), 128.7(\mathrm{CH}), 127.3(\mathrm{CH}), 126.1(\mathrm{CH}), 123.5(\mathrm{CH}), 112.4$ $(\mathrm{CH}), 60.4(\mathrm{CH}), 50.1\left(\mathrm{CH}_{2}\right), 35.6(\mathrm{CH}), 30.2\left(\mathrm{CH}_{2}\right), 28.9\left(\mathrm{CH}_{2}\right), 26.2\left(\mathrm{CH}_{2}\right), 25.6\left(\mathrm{CH}_{2}\right), 25.5\left(\mathrm{CH}_{2}\right)$, $22.4\left(\mathrm{CH}_{3}\right)$. IR (neat): vmax $\left(\mathrm{cm}^{-1}\right)=3198(\mathrm{w}), 2924(\mathrm{w}), 1628(\mathrm{~s}), 1568(\mathrm{~s}), 1431(\mathrm{~s}), 773(\mathrm{~m}), 631$ (s),534 (m), 494 (s), 405 (s). HRMS (ESI): $m / z$ calculated for $\mathrm{C}_{22} \mathrm{H}_{27} \mathrm{BrN}_{3} \mathrm{O}_{2}[\mathrm{M}+\mathrm{H}]^{+}$444.1281, found 444.1266 .

\section{2-( $N$-benzylpropionamido)- $N$-(6-bromopyridin-2-yl)-3,3-dimethyl-butanamide $(9 \mathbf{j})$.}

Following general procedure IV in $\mathrm{MeOH}$ with benzylamine $(109 \mu \mathrm{L}$,<smiles>CCC(=O)N(Cc1ccccc1)C(C(=O)Nc1cccc(Br)n1)C(C)(C)C</smiles>
$1.0 \mathrm{mmol}, 1.0$ equiv), pivaldehyde $(110 \mu \mathrm{L}, 1.0 \mathrm{mmol}, 1.0$ equiv) and propionic acid $(112 \mu \mathrm{L}, 1.5 \mathrm{mmol}, 1.5$ equiv) the Ugi product was obtained as a white solid (395 $\mathrm{mg}, 0.92 \mathrm{mmol}$, 92\%). Flash chromatography: (cyclohexane : EtOAc $=10: 1$ ). m.p.: $160{ }^{\circ} \mathrm{C} .{ }^{1} \mathrm{H}$ NMR $\left(\mathrm{CDCl}_{3}, 500 \mathrm{MHz}\right) \delta 8.69(\mathrm{bs}, 1 \mathrm{H}), 7.69(\mathrm{~d}, J=8.5 \mathrm{~Hz}, 1 \mathrm{H}), 7.37(\mathrm{t}, J=$ $7.8 \mathrm{~Hz}, 1 \mathrm{H}), 7.18-7-04(\mathrm{~m}, 6 \mathrm{H}), 5.36$ (bs, 1H), 5.19 (d, $J=17.5 \mathrm{~Hz}, 1 \mathrm{H}), 4.74$ (d, $J=17.5 \mathrm{~Hz}, 1 \mathrm{H}), 2.39$ $(\mathrm{m}, 1 \mathrm{H}), 2.22(\mathrm{~m}, 1 \mathrm{H}), 1.14(\mathrm{~s}, 9 \mathrm{H}), 1.10(\mathrm{t}, J=7.3 \mathrm{~Hz}, 3 \mathrm{H}) .{ }^{13} \mathrm{C} \mathrm{NMR}\left(\mathrm{CDCl}_{3}, 125 \mathrm{MHz}\right) \delta 176.7\left(\mathrm{C}_{\mathrm{q}}\right)$, $168.3\left(\mathrm{C}_{\mathrm{q}}\right), 150.8\left(\mathrm{C}_{\mathrm{q}}\right), 140.2(\mathrm{CH}), 139.2\left(\mathrm{C}_{\mathrm{q}}\right), 138.1\left(\mathrm{C}_{\mathrm{q}}\right), 128.6(\mathrm{CH}), 126.7(\mathrm{CH}), 125.3(\mathrm{CH}), 123.6$ $(\mathrm{CH}), 112.4(\mathrm{CH}) 49.6\left(\mathrm{CH}_{2}\right), 36.7\left(\mathrm{C}_{\mathrm{q}}\right), 27.6\left(\mathrm{CH}_{3}\right), 27.4\left(\mathrm{CH}_{2}\right), 9.8\left(\mathrm{CH}_{3}\right)$. IR (neat): vmax $\left(\mathrm{cm}^{-1}\right)=$ 
2960 (w), 1630 (s), 1566 (s), 1529 (m), 1431 (s), 1389 (s), 1153 (s), 1128 (s), 789 (s), 731 (s). HRMS (ESI): $m / z$ calculated for $\mathrm{C}_{21} \mathrm{H}_{27} \mathrm{BrN}_{3} \mathrm{O}_{2}[\mathrm{M}+\mathrm{H}]^{+}$432.1281, found 432.1267.

Methyl 3-(N-(1-((6-bromopyridin-2-yl)amino)-3,3-dimethyl-1-oxobutan-2-yl)acetamido)propanoate<smiles>COC(=O)CCN(C(C)=O)C(C(=O)Nc1cccc(Br)n1)C(C)(C)C</smiles>
(9k).

Following general procedure IV in $\mathrm{MeOH}$ on a $3.0 \mathrm{mmol}$ scale with $\beta$ alanine methyl ester (309 mg, $3.0 \mathrm{mmol}, 1.0$ equiv), pivaldehyde $(330 \mu \mathrm{L}$, $3.0 \mathrm{mmol}, 1.0$ equiv) and acetic acid $(216 \mu \mathrm{L}, 3.75 \mathrm{mmol}, 1.25$ equiv) the Ugi product was obtained as an off-white solid (910 $\mathrm{mg}, 2.20 \mathrm{mmol}, 73 \%)$. Flash chromatography: (cyclohexane $:$ EtOAc $=2: 1$ ). The spectra contain minor amounts of EtOAc. m.p.: $187-188{ }^{\circ} \mathrm{C} .{ }^{1} \mathrm{H}$ NMR $\left(\mathrm{CDCl}_{3}, 500 \mathrm{MHz}\right) \delta$ 9.60 (bs, 1H), 8.17 (d, $J=8.0 \mathrm{~Hz}, 1 \mathrm{H}), 7.53(\mathrm{t}, J=8.0 \mathrm{~Hz}, 1 \mathrm{H}), 7.19$ (d, $J=7.5 \mathrm{~Hz}, 1 \mathrm{H}), 5.31$ (bs, 1H), $4.17(\mathrm{~m}, 1 \mathrm{H}), 3.85(\mathrm{~m}, 1 \mathrm{H}), 3.65(\mathrm{~s}, 3 \mathrm{H}), 2.63-2.56(\mathrm{~m}, 2 \mathrm{H}), 2.34(\mathrm{~s}, 3 \mathrm{H}), 1.11(\mathrm{~s}, 9 \mathrm{H}) .{ }^{13} \mathrm{C} \mathrm{NMR}\left(\mathrm{CDCl}_{3}\right.$, $125 \mathrm{MHz}) \delta 172.8\left(\mathrm{C}_{\mathrm{q}}\right), 171.3\left(\mathrm{C}_{\mathrm{q}}\right), 169.1\left(\mathrm{C}_{\mathrm{q}}\right), 151.3\left(\mathrm{C}_{\mathrm{q}}\right), 140.4(\mathrm{CH}), 139.5\left(\mathrm{C}_{\mathrm{q}}\right), 123.7(\mathrm{CH}), 112.6$ $(\mathrm{CH}), 60.4\left(\mathrm{CH}_{2}\right), 51.9(\mathrm{CH}), 36.5\left(\mathrm{C}_{\mathrm{q}}\right), 34.6\left(\mathrm{CH}_{2}\right), 27.8\left(\mathrm{CH}_{3}\right), 21.9\left(\mathrm{CH}_{3}\right)$. IR (neat): vmax $\left(\mathrm{cm}^{-1}\right)=$ 2957 (w), 1735 (s), 1628 (s), 1568 (s), 1431 (s), 1153 (s), 764 (m), 631 (s), 534 (m), 498 (s), 401 (s). HRMS (ESI): $m / z$ calculated for $\mathrm{C}_{17} \mathrm{H}_{25} \mathrm{BrN}_{3} \mathrm{O}_{4}[\mathrm{M}+\mathrm{H}]^{+} 414.1023$, found 414.1017.

\section{General procedure V: Hydrolysis of Ugi products.}

Unless stated otherwise: An Ugi product (9, $0.1 \mathrm{mmol}, 1.0$ equiv) was dissolved in $\mathrm{MeOH}(0.75 \mathrm{~mL})$ and $2 \mathrm{M} \mathrm{NaOH}(0.25 \mathrm{~mL}, 5.0$ equiv) was added. The resulting mixture was stirred for 48 hours at room temperature. The crude mixture was acidified to $\mathrm{pH} 1$ with $1 \mathrm{M} \mathrm{HCl}$ and extracted with EtOAc. The organic layer was washed with $1 \mathrm{M} \mathrm{HCl}$ and with brine, dried over $\mathrm{Na}_{2} \mathrm{SO}_{4}$ and concentrated under reduced pressure to yield the corresponding carboxylic acid 8. Note: rotamers could be observed in the NMR data.

\section{2-( $N$-benzylacetamido)-4-methylpentanoic acid (8a).}<smiles>CC(=O)N(Cc1ccccc1)C(CC(C)C)C(=O)O</smiles>

Following general procedure $\mathbf{V}$ with 2 -( $N$-benzylacetamido)- $N$-(6-bromopyridin-2yl)-4-methylpentanamide (7l, $42 \mathrm{mg}, 0.1 \mathrm{mmol}, 1.0$ equiv) the corresponding carboxylic acid was obtained as an off-white solid (26 mg, $0.1 \mathrm{mmol}, 100 \%)$. m.p.: 90 ${ }^{\circ} \mathrm{C}$. Two rotamers were present on NMR timescale $\left(\mathrm{R}^{1}: \mathrm{R}^{2}=1: 0.3\right)$. ${ }^{1} \mathrm{H}$ NMR $\left(\mathrm{CDCl}_{3}, 500 \mathrm{MHz}\right) \delta 11.40(\mathrm{bs}, 1.3 \mathrm{H}), 7.42-7.18(\mathrm{~m}, 6.5 \mathrm{H}), 5.05(\mathrm{~d}, J=15.6 \mathrm{~Hz}$, $0.3 \mathrm{H}), 4.75-4.70(\mathrm{~m}, 2 \mathrm{H}), 4.51(\mathrm{~d}, J=17.3 \mathrm{~Hz}, 1 \mathrm{H}), 4.42(\mathrm{~m}, 0.3 \mathrm{H}), 4.22(\mathrm{~d}, J=15.6$ $\mathrm{Hz}, 0.3 \mathrm{H}), 2.30(\mathrm{~s}, 0.9 \mathrm{H}), 2.17(\mathrm{~s}, 3 \mathrm{H}), 1.92(\mathrm{~m}, 1 \mathrm{H}), 1.73(\mathrm{~m}, 0.3 \mathrm{H}), 1.65-1.49(\mathrm{~m}, 2.3 \mathrm{H}), 1.36(\mathrm{~m}, 0.3 \mathrm{H})$, $0.89(\mathrm{~d}, J=6.5 \mathrm{~Hz}, 3 \mathrm{H}), 0.86(\mathrm{~d}, J=6.5 \mathrm{~Hz}, 0.9 \mathrm{H}), 0.75(\mathrm{~d}, J=6.6 \mathrm{~Hz}, 3 \mathrm{H}), 0.59(\mathrm{~d}, J=6.7 \mathrm{~Hz}, 0.9 \mathrm{H})$. ${ }^{13} \mathrm{C} \mathrm{NMR}\left(\mathrm{CDCl}_{3}, 125 \mathrm{MHz}\right) \delta 175.4\left(\mathrm{C}_{\mathrm{q}}\right), 174.4\left(\mathrm{C}_{\mathrm{q}}\right), 173.3\left(\mathrm{C}_{\mathrm{q}}\right), 173.0\left(\mathrm{C}_{\mathrm{q}}\right), 138.2\left(\mathrm{C}_{\mathrm{q}}\right), 136.5\left(\mathrm{C}_{\mathrm{q}}\right)$, $128.8(\mathrm{CH}), 128.1(\mathrm{CH}), 127.8(\mathrm{CH}), 127.6(\mathrm{CH}), 126.9(\mathrm{CH}), 126.6(\mathrm{CH}), 59.5(\mathrm{CH}), 57.2(\mathrm{CH}), 51.7$ $\left(\mathrm{CH}_{2}\right), 47.5\left(\mathrm{CH}_{2}\right), 38.4\left(\mathrm{CH}_{2}\right), 38.1\left(\mathrm{CH}_{2}\right), 25.2(\mathrm{CH}), 24.4(\mathrm{CH}), 22.2\left(\mathrm{CH}_{3}\right), 22.2\left(\mathrm{CH}_{3}\right), 22.2\left(\mathrm{CH}_{3}\right)$, $22.0\left(\mathrm{CH}_{3}\right), 22.0\left(\mathrm{CH}_{3}\right), 21.7\left(\mathrm{CH}_{3}\right)$. IR (neat): vmax $\left(\mathrm{cm}^{-1}\right)=2955(\mathrm{w}), 1735(\mathrm{~s}), 1718(\mathrm{~s}), 729(\mathrm{~s}), 632$ (s), $546(\mathrm{~s}), 501$ (s). HRMS (ESI): $m / z$ calculated for $\mathrm{C}_{15} \mathrm{H}_{22} \mathrm{NO}_{3}[\mathrm{M}+\mathrm{H}]^{+} 264.1594$, found 264.1603 . 


\section{2-(N-benzylacetamido)acetic acid (8b).}<smiles>CC(=O)N(CC(=O)O)Cc1ccccc1</smiles>

Following general procedure $\mathbf{V}$ with $N$-benzyl- $N$-(2-((6-bromopyridin-2-yl)amino)2-oxoethyl)acetamide (9b, $36 \mathrm{mg}, 0.10 \mathrm{mmol}, 1.0$ equiv) the corresponding carboxylic acid was obtained as an off-white solid $(21 \mathrm{mg}, 0.10 \mathrm{mmol}, 100 \%)$. Two rotamers were present on NMR timescale $\left(\mathrm{R}^{1}: \mathrm{R}^{2}=1: 0.4\right) .{ }^{1} \mathrm{H}$ NMR $\left(\mathrm{CDCl}_{3}, 500 \mathrm{MHz}\right) \delta$ 8.60 (bs, 1.4H), 7.39-7.19 (m, 7H), $4.66(\mathrm{~s}, 0.8 \mathrm{H}), 4.63(\mathrm{~s}, 2 \mathrm{H}), 4.09(\mathrm{~s}, 2 \mathrm{H}), 3.94(\mathrm{~s}, 0.8 \mathrm{H}), 2.25(\mathrm{~s}, 3 \mathrm{H})$, $2.16(\mathrm{~s}, 1.2 \mathrm{H}) .{ }^{13} \mathrm{C} \mathrm{NMR}\left(\mathrm{CDCl}_{3}, 125 \mathrm{MHz}\right) \delta 172.7\left(\mathrm{C}_{\mathrm{q}}\right), 172.6\left(\mathrm{C}_{\mathrm{q}}\right), 172.3\left(\mathrm{C}_{\mathrm{q}}\right), 172.1\left(\mathrm{C}_{\mathrm{q}}\right), 136.31\left(\mathrm{C}_{\mathrm{q}}\right)$, $135.4\left(\mathrm{C}_{\mathrm{q}}\right), 129.1(\mathrm{CH}), 128.7(\mathrm{CH}), 128.4(\mathrm{CH}), 128.0(\mathrm{CH}), 127.7(\mathrm{CH}), 126.7(\mathrm{CH}), 53.0\left(\mathrm{CH}_{2}\right), 49.5$ $\left(\mathrm{CH}_{2}\right), 48.8\left(\mathrm{CH}_{2}\right), 47.1\left(\mathrm{CH}_{2}\right), 21.3\left(\mathrm{CH}_{3}\right), 21.1\left(\mathrm{CH}_{3}\right)$. IR (neat): vmax $\left(\mathrm{cm}^{-1}\right)=2927(\mathrm{w}), 1732(\mathrm{~m})$, $1599(\mathrm{~m}), 1431$ (m), 1201 (m), 633 (s), $532(\mathrm{~m}), 498$ (s), 403 (s). HRMS (ESI): m/z calculated for $\mathrm{C}_{11} \mathrm{H}_{14} \mathrm{NO}_{3}[\mathrm{M}+\mathrm{H}]^{+}$208.0968, found 208.0964.

\section{2-( $N$-benzylbenzamido)-4-methylpentanoic acid (8c).}<smiles>CC(C)CC(C(=O)O)N(Cc1ccccc1)C(=O)c1ccccc1</smiles>
Following general procedure $\mathbf{V}$ with $N$-benzyl- $N$-(1-((6-bromopyridin-2yl)amino)-4-methyl-1-oxopentan-2-yl)benzamide (9c, $48 \mathrm{mg}, 0.10 \mathrm{mmol}, 1.0$ equiv) the corresponding carboxylic acid was obtained as a brown oil $(33 \mathrm{mg}, 0.10 \mathrm{mmol}$, $100 \%)$. Two rotamers were present on NMR timescale $\left(\mathrm{R}^{1}: \mathrm{R}^{2}=1: 0.7\right) .{ }^{1} \mathrm{H}$ NMR $\left(\mathrm{CDCl}_{3}, 500 \mathrm{MHz}\right) \delta 10.45(\mathrm{bs}, 1.7 \mathrm{H}), 7.59-7.16(\mathrm{~m}, 17 \mathrm{H}), 5.19(\mathrm{~d}, J=14.3 \mathrm{~Hz}$, $0.7 \mathrm{H}), 4.69(\mathrm{~d}, J=15.6 \mathrm{~Hz}, 1 \mathrm{H}), 4.49(\mathrm{~m}, 1.7 \mathrm{H}), 4.21(\mathrm{~d}, J=14.6 \mathrm{~Hz}, 0.7 \mathrm{H}), 4.14$ $(\mathrm{m}, 1 \mathrm{H}), 2.19(\mathrm{~m}, 1 \mathrm{H}), 1.83-1.52(\mathrm{~m}, 3.1 \mathrm{H}), 1.30(\mathrm{~m}, 1 \mathrm{H}), 0.98-0.82(\mathrm{~m}, 6 \mathrm{H}), 0.60-0.40(\mathrm{~m}, 4.2 \mathrm{H}) .{ }^{13} \mathrm{C}$ $\operatorname{NMR}\left(\mathrm{CDCl}_{3}, 125 \mathrm{MHz}\right) \delta 176.0\left(\mathrm{C}_{\mathrm{q}}\right), 175.5\left(\mathrm{C}_{\mathrm{q}}\right), 173.7\left(\mathrm{C}_{\mathrm{q}}\right), 173.6\left(\mathrm{C}_{\mathrm{q}}\right), 138.3\left(\mathrm{C}_{\mathrm{q}}\right), 135.8\left(\mathrm{C}_{\mathrm{q}}\right), 135.7$ $\left(\mathrm{C}_{\mathrm{q}}\right), 135.2\left(\mathrm{C}_{\mathrm{q}}\right), 130.3(\mathrm{CH}), 130.1(\mathrm{CH}), 129.7(\mathrm{CH}), 128.8(\mathrm{CH}), 128.4(\mathrm{CH}), 128.3(\mathrm{CH}), 128.1(\mathrm{CH})$, $127.9(\mathrm{CH}), 127.4(\mathrm{CH}), 127.0(\mathrm{CH}), 126.6(\mathrm{CH}), 60.6(\mathrm{CH}), 58.7(\mathrm{CH}), 54.6\left(\mathrm{CH}_{2}\right), 47.4\left(\mathrm{CH}_{2}\right), 38.2$ $\left(\mathrm{CH}_{2}\right), 29.7\left(\mathrm{CH}_{2}\right), 25.4(\mathrm{CH}), 24.2(\mathrm{CH}), 22.4\left(\mathrm{CH}_{3}\right), 22.2\left(\mathrm{CH}_{3}\right), 21.4\left(\mathrm{CH}_{3}\right)$. IR (neat): vmax $\left(\mathrm{cm}^{-1}\right)=$ 2957 (w), 1713 (m), 1593 (m), 1447 (m), 1246 (m), 733 (m), 633 (s), 536 (s), 494 (s), 403 (s). HRMS (ESI): $m / z$ calculated for $\mathrm{C}_{20} \mathrm{H}_{24} \mathrm{NO}_{3}[\mathrm{M}+\mathrm{H}]^{+} 326.1751$, found 326.1748 .

\section{$N$-benzyl- $N$-(thiophene-2-carbonyl)leucine (8d).}<smiles>CC(C)CC(C(=O)O)N(Cc1ccccc1)C(=O)c1cccs1</smiles>

Following general procedure $\mathbf{V}$ with $N$-benzyl- $N$-(1-((6-bromopyridin-2yl)amino)-4-methyl-1-oxopentan-2-yl)thiophene-2-carboxamide (9d, $49 \mathrm{mg}$, $0.10 \mathrm{mmol}, 1.0$ equiv) the corresponding carboxylic acid was obtained as a yellow oil (33 mg, $0.10 \mathrm{mmol}, 100 \%) .{ }^{1} \mathrm{H} \mathrm{NMR}\left(\mathrm{CDCl}_{3}, 500 \mathrm{MHz}\right) \delta 10.35$ (bs, 1H), 7.49 (s, 1H), 7.47-7.30 (m, 6H), $7.00(\mathrm{~s}, 1 \mathrm{H}), 4.99$ (d, J=16.5 Hz, 1H), 4.79 (bs, 1H), $4.16(\mathrm{bs}, 1 \mathrm{H}), 2.17(\mathrm{~m}, 1 \mathrm{H}), 1.65(\mathrm{~m}, 2 \mathrm{H}), 0.91-0.63(\mathrm{~m}, 6 \mathrm{H}) .{ }^{13} \mathrm{C} \mathrm{NMR}$ $\left(\mathrm{CDCl}_{3}, 125 \mathrm{MHz}\right) \delta 175.8\left(\mathrm{C}_{\mathrm{q}}\right), 166.4\left(\mathrm{C}_{\mathrm{q}}\right), 136.9\left(\mathrm{C}_{\mathrm{q}}\right), 129.5(\mathrm{CH}), 128.8(\mathrm{CH}), 127.8(\mathrm{CH}), 127.4(\mathrm{CH})$, 127.2 $(\mathrm{CH}), 60.0(\mathrm{CH}), 38.3\left(\mathrm{CH}_{2}\right), 29.7\left(\mathrm{CH}_{2}\right), 25.3(\mathrm{CH}), 22.5\left(\mathrm{CH}_{3}\right), 22.1\left(\mathrm{CH}_{3}\right)$. IR (neat): vmax $\left(\mathrm{cm}^{-}\right.$ $\left.{ }^{1}\right)=2957(\mathrm{w}), 1715(\mathrm{~s}), 1591(\mathrm{~m}), 1522(\mathrm{~s}), 1425(\mathrm{~m}), 1352(\mathrm{~m}), 1319(\mathrm{~m}), 1245(\mathrm{~m}), 1173(\mathrm{~m}), 968(\mathrm{~m})$, 908 (m), 858 (m), 729 (s). HRMS (ESI): $\mathrm{m} / z$ calculated for $\mathrm{C}_{18} \mathrm{H}_{22} \mathrm{NO}_{3} \mathrm{~S}[\mathrm{M}+\mathrm{H}]^{+}$332.1315, found 332.1305 . 


\section{$N$-(4-chlorobenzyl)- $N$-pivaloylleucine (8e).}<smiles>CC(C)CC(C(=O)O)N(Cc1ccc(Cl)cc1)C(=O)C(C)(C)C</smiles>

Following general procedure $\mathbf{V}$ with $N$-(6-bromopyridin-2-yl)-2-( $N$-(4chlorobenzyl)pivalamido)-4-methylpentanamide (9e, $50 \mathrm{mg}, 0.10 \mathrm{mmol}, 1.0$ equiv) the corresponding carboxylic acid was obtained as a brown oil (34 mg, $0.10 \mathrm{mmol}, 100 \%) .{ }^{1} \mathrm{H} \mathrm{NMR}\left(\mathrm{CDCl}_{3}, 500 \mathrm{MHz}\right) \delta 7.34(\mathrm{~d}, J=7.4 \mathrm{~Hz}, 1 \mathrm{H})$, $7.28(\mathrm{~d}, J=7.4 \mathrm{~Hz}, 1 \mathrm{H}), 4.91(\mathrm{~d}, J=16.5 \mathrm{~Hz}, 1 \mathrm{H}), 4.55(\mathrm{~d}, J=15.0 \mathrm{~Hz}, 1 \mathrm{H})$, $3.49(\mathrm{~m}, 1 \mathrm{H}), 2.19(\mathrm{~m}, 1 \mathrm{H}), 1.59(\mathrm{~m}, 1 \mathrm{H}), 1.43(\mathrm{~m}, 1 \mathrm{H}), 1.34(\mathrm{~s}, 9 \mathrm{H}), 0.86(\mathrm{~d}$, $J=6.5 \mathrm{~Hz}, 1 \mathrm{H}), 0.73(\mathrm{~d}, J=6.5 \mathrm{~Hz}, 1 \mathrm{H}) .{ }^{13} \mathrm{C} \mathrm{NMR}\left(\mathrm{CDCl}_{3}, 125 \mathrm{MHz}\right) \delta$ 179.6 $\left(\mathrm{C}_{\mathrm{q}}\right), 174.7\left(\mathrm{C}_{\mathrm{q}}\right), 129.0(\mathrm{CH}), 128.9(\mathrm{CH}), 60.6(\mathrm{CH}), 39.3\left(\mathrm{CH}_{2}\right), 38.7\left(\mathrm{C}_{\mathrm{q}}\right), 29.7\left(\mathrm{CH}_{2}\right), 28.4\left(\mathrm{CH}_{3}\right)$, $25.4(\mathrm{CH}), 22.8\left(\mathrm{CH}_{3}\right), 22.0\left(\mathrm{CH}_{3}\right)$. IR (neat): vmax $\left(\mathrm{cm}^{-1}\right)=2959(\mathrm{w}), 1734(\mathrm{~m}), 1716(\mathrm{~m}), 1636(\mathrm{~m})$, 1493 (s), $1182(\mathrm{~m}), 790(\mathrm{~m}), 516(\mathrm{~s}), 430(\mathrm{~s})$. HRMS (ESI): $\mathrm{m} / z$ calculated for $\mathrm{C}_{18} \mathrm{H}_{27} \mathrm{ClNO}_{3}[\mathrm{M}+\mathrm{H}]^{+}$ 340.1674 , found 340.1650 .

\section{2-( $N$-benzylacetamido)-2-phenylacetic acid (8f).}<smiles>CC(=O)N(Cc1ccccc1)C(C(=O)O)c1ccccc1</smiles>
Following general procedure $\mathbf{V}$ with 2 -( $N$-benzylacetamido)- $N$-(6-bromopyridin-2yl)-2-phenylacetamide (9f, $44 \mathrm{mg}, 0.1 \mathrm{mmol}, 1.0$ equiv) the corresponding carboxylic acid was obtained as a yellow-brown solid $(28 \mathrm{mg}, 0.1 \mathrm{mmol}, 100 \%)$. m.p.: $130{ }^{\circ} \mathrm{C}$. ${ }^{1} \mathrm{H} \mathrm{NMR}\left(\mathrm{CDCl}_{3}, 500 \mathrm{MHz}\right) \delta 10.40(\mathrm{bs}, 1 \mathrm{H}), 7.37-7.17(\mathrm{~m}, 8 \mathrm{H}), 6.99(\mathrm{~m}, 2 \mathrm{H}), 5.92$ $(\mathrm{s}, 1 \mathrm{H}), 4.68(\mathrm{~d}, J=17.6 \mathrm{~Hz}, 1 \mathrm{H}), 4.45(\mathrm{~d}, J=17.6 \mathrm{~Hz}, 1 \mathrm{H}), 2.13(\mathrm{~s}, 3 \mathrm{H}) .{ }^{13} \mathrm{C} \mathrm{NMR}$ $\left(\mathrm{CDCl}_{3}, 125 \mathrm{MHz}\right) \delta 173.8\left(\mathrm{C}_{\mathrm{q}}\right), 173.4\left(\mathrm{C}_{\mathrm{q}}\right), 136.7\left(\mathrm{C}_{\mathrm{q}}\right), 133.5\left(\mathrm{C}_{\mathrm{q}}\right), 129.9(\mathrm{CH}), 128.8(\mathrm{CH}), 128.7(\mathrm{CH})$, 128.6 (CH), $127.1(\mathrm{CH}), 126.1(\mathrm{CH}), 62.6(\mathrm{CH}), 50.6\left(\mathrm{CH}_{2}\right), 22.1\left(\mathrm{CH}_{3}\right)$. IR (neat): vmax $\left(\mathrm{cm}^{-1}\right)=2922$ (w), 1732 (m), 1597 (m), 1418 (m), 1204 (m), 729 (s), 698 (s), 634 (s), 530 (s), 496 (s), 401 (s). HRMS (ESI): $m / z$ calculated for $\mathrm{C}_{17} \mathrm{H}_{18} \mathrm{NO}_{3}[\mathrm{M}+\mathrm{H}]^{+}$284.1281, found 284.1274.

\section{2-(N-(4-methoxyphenethyl)propionamido)-2-phenylacetic acid (8g).}

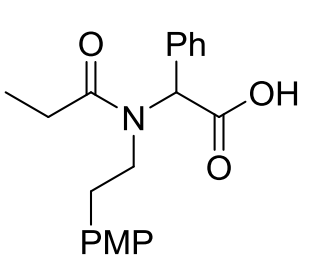

Following general procedure $\mathbf{V}$ with $N$-(2-((6-bromopyridin-2-yl)amino)-2-oxo-1phenylethyl)- $N$-(4-methoxyphenethyl)propionamide $(9 \mathrm{~g}, 50 \mathrm{mg}, 0.10 \mathrm{mmol}, 1.0$ equiv) the corresponding carboxylic acid was obtained as an off-white solid (34 $\mathrm{mg}, 0.10 \mathrm{mmol}, 100 \%)$. Two rotamers were present on NMR timescale $\left(\mathrm{R}^{1}: \mathrm{R}^{2}=1\right.$ : 0.14). ${ }^{1} \mathrm{H} \mathrm{NMR}\left(\mathrm{CDCl}_{3}, 500 \mathrm{MHz}\right) \delta 10.71(\mathrm{bs}, 1.14 \mathrm{H}), 7.42(\mathrm{~m}, 5.7 \mathrm{H}), 6.75(\mathrm{~m}$, $4.56 \mathrm{H}), 6.03(\mathrm{~s}, 1 \mathrm{H}), 5.74(\mathrm{~s}, 0.14 \mathrm{H}), 3.75(\mathrm{~s}, 3.42 \mathrm{H}), 3.65(\mathrm{~m}, 0.14 \mathrm{H}), 3.42(\mathrm{~m}$,

$1 \mathrm{H}), 3.35(\mathrm{~m}, 1 \mathrm{H}), 3.17(\mathrm{~m}, 0.14 \mathrm{H}), 2.75(\mathrm{~m}, 0.14 \mathrm{H}), 2.58(\mathrm{~m}, 1 \mathrm{H}), 2.50-2.31(\mathrm{~m}, 2.28 \mathrm{H}), 2.13(\mathrm{~m}, 1 \mathrm{H})$, $1.92(\mathrm{~m}, 0.14 \mathrm{H}), 1.16(\mathrm{t}, J=7.0 \mathrm{~Hz}, 3.42 \mathrm{H}) .{ }^{13} \mathrm{C} \mathrm{NMR}\left(\mathrm{CDCl}_{3}, 125 \mathrm{MHz}\right) \delta 175.6\left(\mathrm{C}_{\mathrm{q}}\right), 174.0\left(\mathrm{C}_{\mathrm{q}}\right), 158.2$ $\left(\mathrm{C}_{\mathrm{q}}\right), 133.9\left(\mathrm{C}_{\mathrm{q}}\right), 129.9\left(\mathrm{C}_{\mathrm{q}}\right), 129.8(\mathrm{CH}), 129.4(\mathrm{CH}), 128.9(\mathrm{CH}), 113.9(\mathrm{CH}), 62.5(\mathrm{CH}), 55.2\left(\mathrm{CH}_{3}\right)$, $48.4\left(\mathrm{CH}_{2}\right), 35.2\left(\mathrm{CH}_{2}\right), 26.6\left(\mathrm{CH}_{2}\right), 9.4\left(\mathrm{CH}_{3}\right)$. IR (neat): vmax $\left(\mathrm{cm}^{-1}\right)=2937(\mathrm{w}), 1736(\mathrm{~m}), 1610(\mathrm{~m})$, 1512 (s), 1421 (m), 1246 (s), 1178 (s), 1157 (s), 1036 (m), 727 (m), 702 (s). HRMS (ESI): m/z calculated for $\mathrm{C}_{20} \mathrm{H}_{24} \mathrm{NO}_{4}[\mathrm{M}+\mathrm{H}]^{+} 342.1700$, found 342.1687. 
2-(4-Chlorophenyl)-2-( $N$-propylacetamido)acetic acid $(8 \mathrm{~h})$.

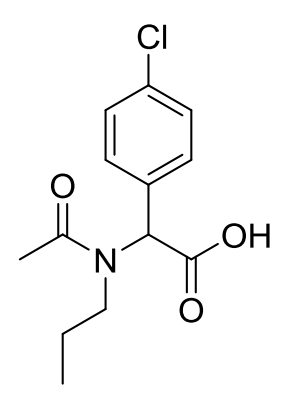

Following general procedure $\mathbf{V}$ at $50{ }^{\circ} \mathrm{C}$ with $\mathrm{N}$-(6-bromopyridin-2-yl)-2-(4chlorophenyl)-2-(N-propylacetamido)-acetamide (9h, $43 \mathrm{mg}, 0.10 \mathrm{mmol}, 1.0$ equiv) the corresponding carboxylic acid was obtained as a colorless oil $(27 \mathrm{mg}, 0.10 \mathrm{mmol}$, $100 \%)$. Two rotamers were present on NMR timescale $\left(\mathrm{R}^{1}: \mathrm{R}^{2}=1: 0.1\right) .{ }^{1} \mathrm{H}$ NMR $\left(\mathrm{CDCl}_{3}, 500 \mathrm{MHz}\right) \delta 10.15(\mathrm{bs}, 1.1 \mathrm{H}), 7.99(\mathrm{~d}, J=7.5 \mathrm{~Hz}, 0.1 \mathrm{H}), 7.42(\mathrm{~d}, J=7.5 \mathrm{~Hz}$, 0.1H), $7.30(\mathrm{~m}, 4 \mathrm{H}), 5.67(\mathrm{~s}, 1 \mathrm{H}), 5.54(\mathrm{~s}, 0.1 \mathrm{H}), 3.37(\mathrm{~m}, 0.1 \mathrm{H}), 3.24(\mathrm{~m}, 1 \mathrm{H}), 3.12$ $(\mathrm{m}, 1 \mathrm{H}), 3.02(\mathrm{~m}, 0.1 \mathrm{H}), 2.20(\mathrm{~s}, 3.3 \mathrm{H}), 1.45(\mathrm{~m}, 1.1 \mathrm{H}), 1.14(\mathrm{~m}, 1.1 \mathrm{H}), 0.73(\mathrm{t}, J=$ $7.3 \mathrm{~Hz}, 3 \mathrm{H}), 0.63(\mathrm{~m}, 0.3 \mathrm{H}) .{ }^{13} \mathrm{C} \mathrm{NMR}\left(\mathrm{CDCl}_{3}, 125 \mathrm{MHz}\right) \delta 172.8\left(\mathrm{C}_{\mathrm{q}}\right), 172.7\left(\mathrm{C}_{\mathrm{q}}\right)$, $172.4\left(\mathrm{C}_{\mathrm{q}}\right), 170.1\left(\mathrm{C}_{\mathrm{q}}\right), 140.1\left(\mathrm{C}_{\mathrm{q}}\right), 134.6\left(\mathrm{C}_{\mathrm{q}}\right), 132.8\left(\mathrm{C}_{\mathrm{q}}\right), 131.5(\mathrm{CH}), 130.8(\mathrm{CH}), 130.2(\mathrm{CH}), 128.8$ $(\mathrm{CH}), 127.9\left(\mathrm{C}_{\mathrm{q}}\right), 64.1(\mathrm{CH}), 62.1(\mathrm{CH}), 49.9\left(\mathrm{CH}_{2}\right), 47.3\left(\mathrm{CH}_{2}\right), 29.7\left(\mathrm{CH}_{2}\right), 22.6\left(\mathrm{CH}_{2}\right), 22.2\left(\mathrm{CH}_{3}\right), 21.4$ $\left(\mathrm{CH}_{3}\right), 11.3\left(\mathrm{CH}_{3}\right), 11.1\left(\mathrm{CH}_{3}\right)$. IR (neat): vmax $\left(\mathrm{cm}^{-1}\right)=2968(\mathrm{w}), 1734(\mathrm{~m}), 1595(\mathrm{~s}), 1493(\mathrm{~s}), 1421(\mathrm{~m})$, 1194 (m), 1092 (s), 1016 (s), 734 (m). HRMS (ESI): $m / z$ calculated for $\mathrm{C}_{13} \mathrm{H}_{17} \mathrm{ClNO}_{3}[\mathrm{M}+\mathrm{H}]^{+} 270.0891$, found 270.0886 .

\section{2-(N-benzylacetamido)-2-cyclohexylacetic acid (8i).}<smiles>CC(=O)N(Cc1ccccc1)C(C(=O)O)C1CCCCC1</smiles>

Following general procedure $\mathbf{V}$ at $50{ }^{\circ} \mathrm{C}$ with 2 -( $N$-benzylacetamido)- $N$-(6bromopyridin-2-yl)-2-cyclohexylacetamide (9i, $44 \mathrm{mg}, 0.10 \mathrm{mmol}, 1.0$ equiv) the corresponding carboxylic acid was obtained as an off-white solid $(29 \mathrm{mg}, 0.10 \mathrm{mmol}$, $100 \%)$. Two rotamers were present on NMR timescale $\left(\mathrm{R}^{1}: \mathrm{R}^{2}=1: 0.2\right) .{ }^{1} \mathrm{H}$ NMR $\left(\mathrm{CDCl}_{3}, 500 \mathrm{MHz}\right) \delta 10.78(\mathrm{bs}, 1.2 \mathrm{H}), 7.38-7.11(\mathrm{~m}, 6 \mathrm{H}), 4.74(\mathrm{~d}, J=16.8 \mathrm{~Hz}, 1 \mathrm{H})$, $4.67(\mathrm{~d}, J=15.5 \mathrm{~Hz}, 0.2 \mathrm{H}), 4.60(\mathrm{~d}, J=15.5 \mathrm{~Hz}, 0.2 \mathrm{H}), 4.40(\mathrm{~d}, J=16.8 \mathrm{~Hz}, 1 \mathrm{H})$, $3.99(\mathrm{~d}, J=11.0 \mathrm{~Hz}, 0.2 \mathrm{H}), 3.87(\mathrm{~d}, J=10.5 \mathrm{~Hz}, 1 \mathrm{H}), 2.32(\mathrm{~m}, 1 \mathrm{H}), 2.26(\mathrm{~s}, 0.6 \mathrm{H}), 2.20(\mathrm{~s}, 3 \mathrm{H}), 1.93(\mathrm{~m}$, $0.2 \mathrm{H}), 1.81-1.56(\mathrm{~m}, 4.8 \mathrm{H}), 1.48(\mathrm{~m}, 0.2 \mathrm{H}), 1.36-1.04(\mathrm{~m}, 4.6 \mathrm{H}), 1.01-0.68(\mathrm{~m}, 2.4 \mathrm{H}) .{ }^{13} \mathrm{C} \mathrm{NMR}\left(\mathrm{CDCl}_{3}\right.$, $125 \mathrm{MHz}) \delta 174.7\left(\mathrm{C}_{\mathrm{q}}\right), 173.5\left(\mathrm{C}_{\mathrm{q}}\right), 172.5\left(\mathrm{C}_{\mathrm{q}}\right), 171.8\left(\mathrm{C}_{\mathrm{q}}\right), 137.9\left(\mathrm{C}_{\mathrm{q}}\right), 135.2\left(\mathrm{C}_{\mathrm{q}}\right), 129.0(\mathrm{CH}), 128.1$ $(\mathrm{CH}), 128.0(\mathrm{CH}), 127.9(\mathrm{CH}), 126.8(\mathrm{CH}), 126.7(\mathrm{CH}), 70.6(\mathrm{CH}), 66.2(\mathrm{CH}), 55.4\left(\mathrm{CH}_{2}\right), 46.2\left(\mathrm{CH}_{2}\right)$, $36.7(\mathrm{CH}), 36.0(\mathrm{CH}), 30.3\left(\mathrm{CH}_{2}\right), 30.1\left(\mathrm{CH}_{2}\right), 29.7\left(\mathrm{CH}_{2}\right), 29.3\left(\mathrm{CH}_{2}\right), 26.1\left(\mathrm{CH}_{2}\right), 26.0\left(\mathrm{CH}_{2}\right), 25.6$ $\left(\mathrm{CH}_{2}\right), 25.5\left(\mathrm{CH}_{2}\right), 25.3\left(\mathrm{CH}_{2}\right), 22.6\left(\mathrm{CH}_{3}\right), 22.3\left(\mathrm{CH}_{3}\right)$. IR (neat): vmax $\left(\mathrm{cm}^{-1}\right)=2926(\mathrm{~m}), 1728(\mathrm{~m})$, 1601 (s), 1450 (s), 1421 (m), 1240 (m), 1184 (m), 910 (m), 729 (s), 696 (s). HRMS (ESI): m/z calculated for $\mathrm{C}_{17} \mathrm{H}_{24} \mathrm{NO}_{3}[\mathrm{M}+\mathrm{H}]^{+} 290.1751$, found 290.1733 .

\section{2-( $N$-benzylpropionamido)-3,3-dimethylbutanoic acid (8j).}<smiles>CCC(=O)N(Cc1ccccc1)C(C(=O)O)C(C)(C)C</smiles>

Following general procedure $\mathbf{V}$ at $50{ }^{\circ} \mathrm{C}$ with 2-(N-benzylpropionamido)-N-(6bromopyridin-2-yl)-3,3-dimethylbutanamide (9j, $43 \mathrm{mg}, 0.10 \mathrm{mmol}, 1.0$ equiv) the corresponding carboxylic acid was obtained as a white solid $(28 \mathrm{mg}, 0.10 \mathrm{mmol}$, 100\%). ${ }^{1} \mathrm{H} \mathrm{NMR}\left(\mathrm{CDCl}_{3}, 500 \mathrm{MHz}\right) \delta 7.35(\mathrm{t}, J=7.3 \mathrm{~Hz}, 2 \mathrm{H}), 7.28(\mathrm{~d}, J=7.3 \mathrm{~Hz}$, $1 \mathrm{H}), 7.16(\mathrm{~d}, J=7.3 \mathrm{~Hz}, 2 \mathrm{H}), 4.96(\mathrm{~d}, J=17.0 \mathrm{~Hz}, 1 \mathrm{H}), 4.40(\mathrm{~d}, J=17.0 \mathrm{~Hz}, 1 \mathrm{H})$, 4.29 (bs, 1H), $2.37(\mathrm{~m}, 2 \mathrm{H}), 1.12(\mathrm{~m}, 12 \mathrm{H}) .{ }^{13} \mathrm{C} \mathrm{NMR}\left(\mathrm{CDCl}_{3}, 125 \mathrm{MHz}\right) \delta 178.2\left(\mathrm{C}_{\mathrm{q}}\right), 171.4\left(\mathrm{C}_{\mathrm{q}}\right), 136.0$ $\left(\mathrm{C}_{\mathrm{q}}\right), 129.1(\mathrm{CH}), 127.8(\mathrm{CH}), 126.0(\mathrm{CH}), 36.9\left(\mathrm{CH}_{2}\right), 29.7\left(\mathrm{C}_{\mathrm{q}}\right), 28.4\left(\mathrm{CH}_{3}\right), 28.0\left(\mathrm{CH}_{2}\right), 9.5\left(\mathrm{CH}_{3}\right)$. IR (neat): vmax $\left(\mathrm{cm}^{-1}\right)=2961(\mathrm{w}), 1733(\mathrm{~m}), 1604(\mathrm{~m}), 1465(\mathrm{~m}), 1452(\mathrm{~m}), 1213(\mathrm{~m}), 1163(\mathrm{~s}), 966(\mathrm{~m})$, 731 (s), 696 (s). HRMS (ESI): $m / z$ calculated for $\mathrm{C}_{16} \mathrm{H}_{24} \mathrm{NO}_{3}[\mathrm{M}+\mathrm{H}]^{+}$278.1751, found 278.1742. 


\section{2-(N-(2-carboxyethyl)acetamido)-3,3-dimethylbutanoic acid (8k).}

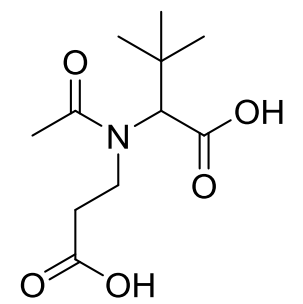

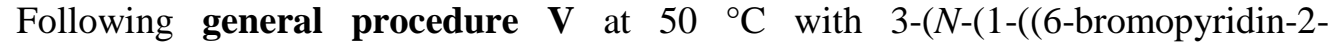
yl)amino)-3,3-dimethyl-1-oxobutan-2-yl)acetamido)propanoate (9k, $41 \mathrm{mg}, 0.10$ mmol, 1.0 equiv) the corresponding carboxylic acid was obtained as a white solid (25 $\mathrm{mg}, 0.10 \mathrm{mmol}, 100 \%)$. Two rotamers were present on NMR timescale $\left(\mathrm{R}^{1}: \mathrm{R}^{2}=1\right.$ : 0.5). ${ }^{1} \mathrm{H}$ NMR (MeOD, $\left.500 \mathrm{MHz}\right) \delta 4.26(\mathrm{bs}, 0.5 \mathrm{H}), 3.95(\mathrm{~m}, 1 \mathrm{H}), 3.63(\mathrm{~m}, 2 \mathrm{H})$, 2.82-2.58 (m, 3H), 2.17 (s, 4.5H), 1.14 (s, 4.5H), $1.10(\mathrm{~s}, 9 \mathrm{H}) .{ }^{13} \mathrm{C}$ NMR (MeOD, 125 MHz) $\delta 175.6\left(\mathrm{C}_{\mathrm{q}}\right), 174.9\left(\mathrm{C}_{\mathrm{q}}\right), 174.9\left(\mathrm{C}_{\mathrm{q}}\right), 174.1\left(\mathrm{C}_{\mathrm{q}}\right), 172.5\left(\mathrm{C}_{\mathrm{q}}\right), 172.2\left(\mathrm{C}_{\mathrm{q}}\right), 69.6(\mathrm{CH}), 69.5(\mathrm{CH}), 42.9$ $\left(\mathrm{CH}_{2}\right), 37.1\left(\mathrm{CH}_{2}\right), 36.6\left(\mathrm{CH}_{2}\right), 34.6\left(\mathrm{CH}_{2}\right), 33.2\left(\mathrm{C}_{\mathrm{q}}\right), 30.8\left(\mathrm{C}_{\mathrm{q}}\right), 28.5\left(\mathrm{CH}_{3}\right), 28.3\left(\mathrm{CH}_{3}\right), 22.7\left(\mathrm{CH}_{3}\right), 21.7$ $\left(\mathrm{CH}_{3}\right)$. IR (neat): vmax $\left(\mathrm{cm}^{-1}\right)=2962(\mathrm{w}), 1717(\mathrm{~m}), 1607(\mathrm{~m}), 1419(\mathrm{~m}), 1367(\mathrm{~s}), 1209(\mathrm{~m}), 1165(\mathrm{~s})$, 797 (s), 540 (m). HRMS (ESI): $m / z$ calculated for $\mathrm{C}_{11} \mathrm{H}_{20} \mathrm{NO}_{5}[\mathrm{M}+\mathrm{H}]^{+}$246.1336, found 246.1327.

\section{General procedure VI: Synthesis of Passerini products (11).}

An aldehyde (1.0 mmol, 1.0 equiv) was dissolved in $\mathrm{CH}_{2} \mathrm{Cl}_{2}(3 \mathrm{~mL})$, acetic acid ( $86 \mu \mathrm{L}, 1.5 \mathrm{mmol}, 1.5$ equiv) and 2-bromo-6-isocyanopyridine (61, $201 \mathrm{mg}, 1.1 \mathrm{mmol}, 1.1$ equiv) were added subsequently. Additional $\mathrm{CH}_{2} \mathrm{Cl}_{2}(1 \mathrm{~mL})$ was added and the resulting mixture was stirred for 48 hours at room temperature. The solvent was removed under reduced pressure and the crude mixture was purified by flash chromatography (cyclohexane : EtOAc) to yield the Passerini products 11.

\section{1-((6-Bromopyridin-2-yl)amino)-3-methyl-1-oxobutan-2-yl acetate (11a).}

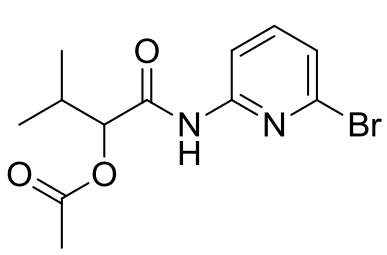

Following general procedure VI with isobutyraldehyde $(91 \mu \mathrm{L}, 1.0 \mathrm{mmol}$, 1.0 equiv) the Passerini product was obtained as an off-white solid (309 mg, 0.98 mmol, 98\%). Flash chromatography: (cyclohexane : EtOAc $=6: 1$ ). m.p.: $99{ }^{\circ} \mathrm{C} .{ }^{1} \mathrm{H} \mathrm{NMR}\left(\mathrm{CDCl}_{3}, 500 \mathrm{MHz}\right) \delta 8.29(\mathrm{bs}, 1 \mathrm{H}), 8.21(\mathrm{~d}, J=8.0 \mathrm{~Hz}, 1 \mathrm{H})$, $7.57(\mathrm{t}, J=8.0 \mathrm{~Hz}, 1 \mathrm{H}), 7.24(\mathrm{~d}, J=8.0 \mathrm{~Hz}, 1 \mathrm{H}), 5.17(\mathrm{~d}, J=4.5 \mathrm{~Hz}, 1 \mathrm{H})$, 2.36-2.29 (m, 1H), 2.24 (s, 3H), $1.02(\mathrm{~d}, J=3.8 \mathrm{~Hz}, 3 \mathrm{H}), 0.99(\mathrm{~d}, J=3.6 \mathrm{~Hz}$, 3H). ${ }^{13} \mathrm{C}$ NMR $\left(\mathrm{CDCl}_{3}, 125 \mathrm{MHz}\right) \delta 169.7\left(\mathrm{C}_{\mathrm{q}}\right), 168.2\left(\mathrm{C}_{\mathrm{q}}\right), 150.5\left(\mathrm{C}_{\mathrm{q}}\right), 140.7(\mathrm{CH}), 139.3\left(\mathrm{C}_{\mathrm{q}}\right), 124.1$ $(\mathrm{CH}), 112.5(\mathrm{CH}), 78.1(\mathrm{CH}), 31.0\left(\mathrm{CH}_{3}\right), 21.0(\mathrm{CH}), 18.6\left(\mathrm{CH}_{3}\right), 17.1\left(\mathrm{CH}_{3}\right)$. IR (neat): vmax $\left(\mathrm{cm}^{-1}\right)=$ 3296 (w), 1743 (m), 1708 (s), 1566 (s), 1519 (m), 1429 (s), 1389 (m), 1232 (m), 789 (m). HRMS (ESI): $m / z$ calculated for $\mathrm{C}_{12} \mathrm{H}_{16} \mathrm{BrN}_{2} \mathrm{O}_{3}[\mathrm{M}+\mathrm{H}]^{+} 315.0339$, found 315.0314 .

\section{1-((6-Bromopyridin-2-yl)amino)-1-oxooctan-2-yl acetate (11b).}

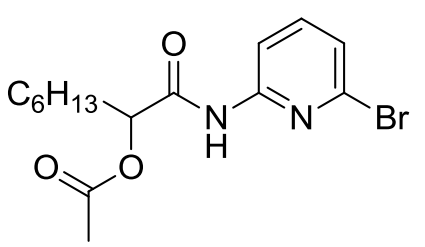

Following general procedure VI with heptanal $(140 \mu \mathrm{L}, 1.0 \mathrm{mmol}, 1.0$ equiv) the Passerini product was obtained as a dark yellow oil $(288 \mathrm{mg}$, $0.81 \mathrm{mmol}, 81 \%)$. Flash chromatography: (cyclohexane : EtOAc $=7: 1) .{ }^{1} \mathrm{H}$ NMR $\left(\mathrm{CDCl}_{3}, 500 \mathrm{MHz}\right) \delta 8.35(\mathrm{bs}, 1 \mathrm{H}), 8.20(\mathrm{~d}, J=8.0 \mathrm{~Hz}, 1 \mathrm{H}), 7.57(\mathrm{t}, J$ $=8.0 \mathrm{~Hz}, 1 \mathrm{H}), 7.25(\mathrm{~d}, J=7.5 \mathrm{~Hz}, 1 \mathrm{H}), 5.26(\mathrm{t}, J=6.0 \mathrm{~Hz}), 2.23(\mathrm{~s}, 3 \mathrm{H})$, $1.90(\mathrm{~m}, 2 \mathrm{H}), 1.37-1.27(\mathrm{~m}, 8 \mathrm{H}), 0.87(\mathrm{t}, J=7.0 \mathrm{~Hz}, 3 \mathrm{H}) .{ }^{13} \mathrm{C} \mathrm{NMR}\left(\mathrm{CDCl}_{3}, 125 \mathrm{MHz}\right) \delta 169.6\left(\mathrm{C}_{\mathrm{q}}\right)$, $168.7\left(\mathrm{C}_{\mathrm{q}}\right), 150.6\left(\mathrm{C}_{\mathrm{q}}\right), 140.7(\mathrm{CH}), 139.3\left(\mathrm{C}_{\mathrm{q}}\right), 124.1(\mathrm{CH}), 112.6(\mathrm{CH}), 74.1(\mathrm{CH}), 32.0\left(\mathrm{CH}_{2}\right), 31.5$ $\left(\mathrm{CH}_{2}\right), 28.8\left(\mathrm{CH}_{2}\right), 24.6\left(\mathrm{CH}_{2}\right), 22.5\left(\mathrm{CH}_{2}\right), 21.1\left(\mathrm{CH}_{3}\right), 14.0\left(\mathrm{CH}_{3}\right)$. IR (neat): vmax $\left(\mathrm{cm}^{-1}\right)=2928(\mathrm{w})$, 1717 (m), 1568 (s), 1431 (s), 787 (m), 631 (s), 536 (s), 498 (s). HRMS (ESI): m/z calculated for $\mathrm{C}_{15} \mathrm{H}_{22} \mathrm{BrN}_{2} \mathrm{O}_{3}[\mathrm{M}+\mathrm{H}]^{+}$357.0808, found 357.0800. 


\section{General procedure VII: Hydrolysis of the Passerini products.}

A Passerini product (11, $0.2 \mathrm{mmol}, 1.0$ equiv) was dissolved in $\mathrm{MeOH}(0.9 \mathrm{~mL})$ and $10 \mathrm{M} \mathrm{NaOH}(0.1 \mathrm{~mL}$, $1 \mathrm{mmol}, 5.0$ equiv) was added. The resulting mixture stirred at room temperature for 48 hours. A small portion of water was added to the crude reaction mixture. The water later was washed with EtOAc $(2 \times)$, acidified to $\mathrm{pH} 1$ with $1 \mathrm{M} \mathrm{HCl}$ and extracted with EtOAc $(4 \times)$. The organic layer was dried over $\mathrm{Na}_{2} \mathrm{SO}_{4}$ and concentrated under reduced pressure to yield the corresponding $\alpha$-hydroxy carboxylic acids $\mathbf{1 2}$.

\section{2-Hydroxy-3-methylbutanoic acid (12a).}

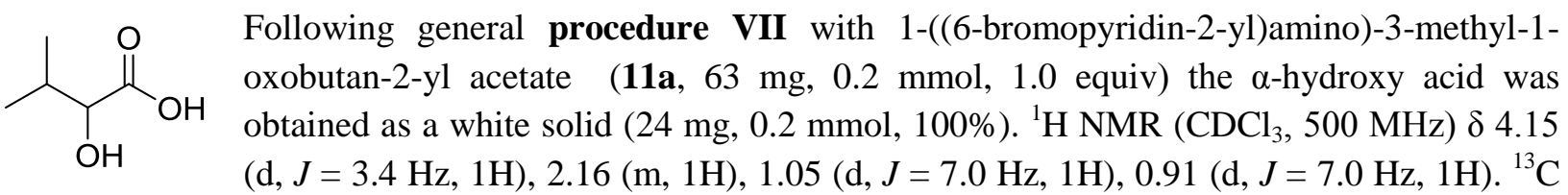
NMR $\left(\mathrm{CDCl}_{3}, 125 \mathrm{MHz}\right) \delta 179.3\left(\mathrm{C}_{\mathrm{q}}\right), 74.8(\mathrm{CH}), 31.9(\mathrm{CH}), 18.8\left(\mathrm{CH}_{3}\right), 15.9\left(\mathrm{CH}_{3}\right)$.

\section{2-Hydroxyoctanoic acid (12b).}<smiles>O=C(O)C(O)c1ccccc1</smiles>

Following general procedure VII with $N$-(6-bromopyridin-2-yl)-2hydroxyoctanamide (11b, $72 \mathrm{mg}, 0.2 \mathrm{mmol}, 1.0$ equiv) the $\alpha$-hydroxy acid was obtained as a white solid (32 mg, $0.2 \mathrm{mmol}, 100 \%) .{ }^{1} \mathrm{H} \mathrm{NMR}\left(\mathrm{CDCl}_{3}, 500 \mathrm{MHz}\right) \delta$ $4.28(\mathrm{dd}, J=7.5,4.2 \mathrm{~Hz}, 1 \mathrm{H}), 1.87(\mathrm{~m}, 1 \mathrm{H}), 1.72(\mathrm{~m}, 1 \mathrm{H}), 1.47-1.41(\mathrm{~m}, 8 \mathrm{H}) 0.88(\mathrm{t}, \mathrm{J}$ $=6.6 \mathrm{~Hz}, 3 \mathrm{H}) .{ }^{13} \mathrm{C} \mathrm{NMR}\left(\mathrm{CDCl}_{3}, 125 \mathrm{MHz}\right) \delta 179.6\left(\mathrm{C}_{\mathrm{q}}\right), 70.2(\mathrm{CH}), 34.2\left(\mathrm{CH}_{2}\right), 31.6\left(\mathrm{CH}_{2}\right), 28.9\left(\mathrm{CH}_{2}\right)$, $24.7\left(\mathrm{CH}_{2}\right), 22.5\left(\mathrm{CH}_{2}\right), 14.0\left(\mathrm{CH}_{3}\right)$.

\section{General procedure VIII: Transacylation under basic conditions.}

To a flame dried Schlenk $3 \AA \mathrm{MS}$, NaOMe in $\mathrm{MeOH}(0.5 \mathrm{M}, 5 \mathrm{~mL}, 2.5 \mathrm{mmol}, 5.0$ equiv) and a nucleophile ( $2.5 \mathrm{mmol}, 5.0$ equiv) were added and predried for 1 hour under nitrogen atmosphere. The Ugi product 2-(N-benzylacetamido)-N-(6-bromopyridin-2-yl)-4-methylpenta-namide (7l, $209 \mathrm{mg}, 0.5$ mmol, 1.0 equiv) was added and the mixture was stirred for $24 \mathrm{~h}$ at $50{ }^{\circ} \mathrm{C}$. The crude mixture was cooled to $0{ }^{\circ} \mathrm{C}$ and acidified at once to $\mathrm{pH} 1$ with $6 \mathrm{M} \mathrm{HCl}$ and extracted with EtOAc. The organic layer was washed with $1 \mathrm{M} \mathrm{HCl}$ and brine, dried over $\mathrm{Na}_{2} \mathrm{SO}_{4}$ and concentrated under reduced pressure. The crude mixture was purified by flash chromatography (cyclohexane : EtOAc) to yield the corresponding products 10.

\section{Methyl 2-( $N$-benzylacetamido)-4-methylpentanoate (10a).}

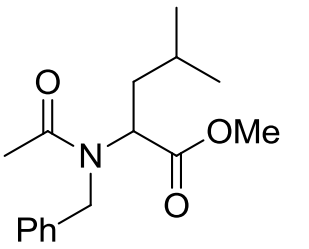

Following general procedure VIII with methanol $(101 \mu \mathrm{L}, 2.5 \mathrm{mmol}, 5.0$ equiv) methyl 2-( $N$-benzylacetamido)-4-methylpentanoate was obtained as a colorless oil (126 mg, $0.46 \mathrm{mmol}, 91 \%)$. Flash chromatography: (cyclohexane : EtOAc =1:2). Or following general procedure IX with methanol $(101 \mu \mathrm{L}, 2.5 \mathrm{mmol}, 5.0$ equiv) methyl ester was obtained as a slightly yellow oil (139 mg, $0.5 \mathrm{mmol}, 100 \%)$. Two rotamers were present on NMR timescale $\left(\mathrm{R}^{1}: \mathrm{R}^{2}=1: 0.35\right) .{ }^{1} \mathrm{H} \mathrm{NMR}\left(\mathrm{CDCl}_{3}, 500 \mathrm{MHz}\right) \delta 7.42-7.17$ $(\mathrm{m}, 6.75 \mathrm{H}), 4.95(\mathrm{t}, J=7.0 \mathrm{~Hz}, 1 \mathrm{H}), 4.66(\mathrm{~d}, J=17.6 \mathrm{~Hz}, 1.35 \mathrm{H}), 5.51(\mathrm{~d}, J=17.6 \mathrm{~Hz}, 1.35 \mathrm{H}), 4.41(\mathrm{dd}$, $J=8.5,6.1 \mathrm{~Hz}, 0.35 \mathrm{H}), 3.60(\mathrm{~s}, 3 \mathrm{H}), 3.49(\mathrm{~s}, 1.05 \mathrm{H}), 2.28(\mathrm{~s}, 1.05 \mathrm{H}), 2.13(\mathrm{~s}, 3 \mathrm{H}), 1.86-1.74(\mathrm{~m}, 1.35 \mathrm{H})$, $1.66(\mathrm{~m}, 0.35 \mathrm{H}), 1.54(\mathrm{~m}, 2 \mathrm{H}), 1.41(\mathrm{~m}, 0.35 \mathrm{H}), 0.90(\mathrm{~m}, 4.05 \mathrm{H}), 0.79(\mathrm{~d}, J=6.3 \mathrm{~Hz}, 3 \mathrm{H}), 0.71(\mathrm{~d}, J=$ $6.7 \mathrm{~Hz}, 1.05 \mathrm{H}) .{ }^{13} \mathrm{C} \mathrm{NMR}\left(\mathrm{CDCl}_{3}, 125 \mathrm{MHz}\right) \delta 172.2\left(\mathrm{C}_{\mathrm{q}}\right), 171.9\left(\mathrm{C}_{\mathrm{q}}\right), 171.7\left(\mathrm{C}_{\mathrm{q}}\right), 171.3\left(\mathrm{C}_{\mathrm{q}}\right), 138.0\left(\mathrm{C}_{\mathrm{q}}\right)$, $137.0\left(\mathrm{C}_{\mathrm{q}}\right), 128.6(\mathrm{CH}), 128.1(\mathrm{CH}), 128.0(\mathrm{CH}), 127.4(\mathrm{CH}), 127.0(\mathrm{CH}), 126.4(\mathrm{CH}), 58.8(\mathrm{CH}), 55.6$ 
$(\mathrm{CH}), 52.1\left(\mathrm{CH}_{3}\right), 51.9\left(\mathrm{CH}_{3}\right), 50.5\left(\mathrm{CH}_{2}\right), 46.2\left(\mathrm{CH}_{2}\right), 38.3\left(\mathrm{CH}_{2}\right), 29.6\left(\mathrm{CH}_{2}\right), 25.1(\mathrm{CH}), 24.3(\mathrm{CH})$, $22.4\left(\mathrm{CH}_{3}\right), 22.4\left(\mathrm{CH}_{3}\right), 22.2\left(\mathrm{CH}_{3}\right), 22.2\left(\mathrm{CH}_{3}\right), 22.1\left(\mathrm{CH}_{3}\right), 21.9\left(\mathrm{CH}_{3}\right)$. IR (neat): vmax $\left(\mathrm{cm}^{-1}\right)=2955$ (m), 1738 (s), 1649 (s), 1410 (s), 1200 (s), 729 (s), 633 (s), 498 (s). HRMS (ESI): m/z calculated for $\mathrm{C}_{16} \mathrm{H}_{24} \mathrm{NO}_{3}[\mathrm{M}+\mathrm{H}]^{+}$278.1751, found 278.1742.

\section{2-( $N$-Benzylacetamido)- $N$-butyl-4-methylpentanamide (10b).}<smiles>CCCCNC(=O)C(CC(C)C)N(Cc1ccccc1)C(C)=O</smiles>

Following general procedure VIII with butylamine $(247 \mu \mathrm{L}, 2.5 \mathrm{mmol}, 5.0$ equiv) the corresponding amide was obtained as a colorless oil (97 mg, $0.31 \mathrm{mmol}, 61 \%)$. Flash chromatography: (cyclohexane $:$ EtOAc $=3: 1$ ). Two rotamers were present on NMR timescale $\left(\mathrm{R}^{1}: \mathrm{R}^{2}=1: 0.1\right) .{ }^{1} \mathrm{H} \mathrm{NMR}\left(\mathrm{CDCl}_{3}, 500 \mathrm{MHz}\right) \delta 7.32(\mathrm{t}, J=7.5$ $\mathrm{Hz}, 2 \mathrm{H}), 7.26(\mathrm{t}, J=7.5 \mathrm{~Hz}, 1 \mathrm{H}), 7.17(\mathrm{~d}, J=7.5 \mathrm{~Hz}, 2 \mathrm{H}), 6.56$ (bs, 1H), 5.04 (m, $1 \mathrm{H}), 4.65(\mathrm{~d}, J=17.8 \mathrm{~Hz}, 1 \mathrm{H}), 4.59(\mathrm{~d}, J=17.8 \mathrm{~Hz}, 1 \mathrm{H}), 3.23-3.11(\mathrm{~m}, 2 \mathrm{H}), 2.06$ $(\mathrm{s}, 3 \mathrm{H}), 1.84(\mathrm{~m}, 1 \mathrm{H}), 1.51-1.34(\mathrm{~m}, 4 \mathrm{H}), 1.33-1.28(\mathrm{~m}, 2 \mathrm{H}), 0.92(\mathrm{t}, J=7.5 \mathrm{~Hz}, 3 \mathrm{H}), 0.86(\mathrm{~d}, J=6.6 \mathrm{~Hz}$, $3 \mathrm{H}), 0.84(\mathrm{~d}, J=6.6 \mathrm{~Hz}, 3 \mathrm{H}) .{ }^{13} \mathrm{C} \mathrm{NMR}\left(\mathrm{CDCl}_{3}, 125 \mathrm{MHz}\right) \delta 172.9\left(\mathrm{C}_{\mathrm{q}}\right), 170.6\left(\mathrm{C}_{\mathrm{q}}\right), 137.5\left(\mathrm{C}_{\mathrm{q}}\right), 128.6$ $(\mathrm{CH}), 127.2(\mathrm{CH}), 125.9(\mathrm{CH}), 55.7(\mathrm{CH}), 48.9\left(\mathrm{CH}_{2}\right), 38.9\left(\mathrm{CH}_{2}\right), 37.0\left(\mathrm{CH}_{2}\right), 31.4\left(\mathrm{CH}_{2}\right), 25.1(\mathrm{CH})$, $22.8\left(\mathrm{CH}_{3}\right), 22.4\left(\mathrm{CH}_{3}\right), 22.3\left(\mathrm{CH}_{3}\right), 20.0\left(\mathrm{CH}_{2}\right), 13.7\left(\mathrm{CH}_{3}\right)$. IR (neat): vmax $\left(\mathrm{cm}^{-1}\right)=3298(\mathrm{~m}), 2957(\mathrm{~m})$, 1630 (s), 1547 (s), 633 (s), 532 (s), 498 (s). HRMS (ESI): $\mathrm{m} / \mathrm{z}$ calculated for $\mathrm{C}_{19} \mathrm{H}_{31} \mathrm{~N}_{2} \mathrm{O}_{2}[\mathrm{M}+\mathrm{H}]^{+}$ 319.2380, found 319.2369.

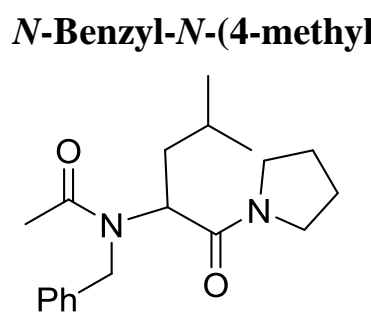

l-1-oxo-1-(pyrrolidin-1-yl)pentan-2-yl)acetamide (10c).

Following general procedure VIII with pyrrolidine $(209 \mu \mathrm{L}, 2.5 \mathrm{mmol}, 5.0$ equiv) the corresponding amide was obtained as an off-white solid (111 mg, 0.35 mmol, 70\%). Flash chromatography: (cyclohexane : EtOAc $=3: 1$ ). Two rotamers were present on NMR timescale $\left(\mathrm{R}^{1}: \mathrm{R}^{2}=1: 0.16\right) .{ }^{1} \mathrm{H} \mathrm{NMR}\left(\mathrm{CDCl}_{3}, 500 \mathrm{MHz}\right)$ $\delta 7.39-7.08(\mathrm{~m}, 5.8 \mathrm{H}), 5.58(\mathrm{t}, J=7.0 \mathrm{~Hz}, 1 \mathrm{H}), 5.08(\mathrm{~d}, J=15.0 \mathrm{~Hz}, 0.16 \mathrm{H}), 4.73$ $(\mathrm{d}, J=17.5 \mathrm{~Hz}, 1 \mathrm{H}), 4.68(\mathrm{~d}, J=17.5 \mathrm{~Hz}, 1 \mathrm{H}), 4.27(\mathrm{~m}, 0.16 \mathrm{H}), 4.05(\mathrm{~d}, J=15.0$ $\mathrm{Hz}, 0.16 \mathrm{H}), 3.71(\mathrm{~m}, 1 \mathrm{H}), 3.51(\mathrm{~m}, 1 \mathrm{H}), 3.35(\mathrm{~m}, 1 \mathrm{H}), 3.21(\mathrm{~m}, 0.32 \mathrm{H}), 3.12(\mathrm{~m}, 0.16 \mathrm{H}), 3.00(\mathrm{~m}, 1 \mathrm{H})$, $2.70(\mathrm{~m}, 0.16 \mathrm{H}), 2.35(\mathrm{~m}, 0.16 \mathrm{H}), 2.27(\mathrm{~s}, 0.48 \mathrm{H}), 2.07(\mathrm{~s}, 3 \mathrm{H}), 2.00-1.69(\mathrm{~m}, 5.64 \mathrm{H}), 1.63-1.41(\mathrm{~m}$, 2.32H), 0.99-0.80 (m, 6.96H). ${ }^{13} \mathrm{C} \mathrm{NMR}\left(\mathrm{CDCl}_{3}, 125 \mathrm{MHz}\right) \delta 171.9\left(\mathrm{C}_{\mathrm{q}}\right), 170.7\left(\mathrm{C}_{\mathrm{q}}\right), 168.9\left(\mathrm{C}_{\mathrm{q}}\right), 166.0$ $\left(\mathrm{C}_{\mathrm{q}}\right), 138.4\left(\mathrm{C}_{\mathrm{q}}\right), 138.0\left(\mathrm{C}_{\mathrm{q}}\right), 128.4(\mathrm{CH}), 128.4(\mathrm{CH}), 127.8(\mathrm{CH}), 126.9(\mathrm{CH}), 126.8(\mathrm{CH}), 125.8(\mathrm{CH})$, $57.8(\mathrm{CH}), 52.1(\mathrm{CH}), 47.9\left(\mathrm{CH}_{2}\right), 46.4\left(\mathrm{CH}_{2}\right), 45.7\left(\mathrm{CH}_{2}\right), 45.6\left(\mathrm{CH}_{2}\right), 45.4\left(\mathrm{CH}_{2}\right), 45.1\left(\mathrm{CH}_{2}\right), 39.2$ $\left(\mathrm{CH}_{2}\right), 38.6\left(\mathrm{CH}_{2}\right), 25.9\left(\mathrm{CH}_{2}\right), 25.6\left(\mathrm{CH}_{2}\right), 24.8(\mathrm{CH}), 24.6(\mathrm{CH}), 24.0\left(\mathrm{CH}_{2}\right), 23.4\left(\mathrm{CH}_{3}\right), 23.4\left(\mathrm{CH}_{2}\right)$, $22.9\left(\mathrm{CH}_{3}\right), 22.6\left(\mathrm{CH}_{3}\right), 22.3\left(\mathrm{CH}_{3}\right), 21.8\left(\mathrm{CH}_{3}\right), 21.7\left(\mathrm{CH}_{3}\right)$. IR (neat): vmax $\left(\mathrm{cm}^{-1}\right)=2955(\mathrm{~m}), 1636(\mathrm{~s})$, $1439(\mathrm{~m}), 1408(\mathrm{~m}), 698(\mathrm{~m}), 621(\mathrm{~m})$. HRMS (ESI): $\mathrm{m} / z$ calculated for $\mathrm{C}_{19} \mathrm{H}_{29} \mathrm{~N}_{2} \mathrm{O}_{2}[\mathrm{M}+\mathrm{H}]^{+} 317.2224$, found 317.2200 .

\section{General procedure IX: Transacylation under acidic conditions.}

Unless stated otherwise: The Ugi product 2-( $N$-benzylacetamido)- $N$-(6-bromopyridin-2-yl)-4-methylpentanamide (7l, $209 \mathrm{mg}, 0.5 \mathrm{mmol}, 1.0$ equiv) was dissolved in $\mathrm{CH}_{2} \mathrm{Cl}_{2}(2 \mathrm{~mL})$ and $\mathrm{HCl}$ in $\mathrm{Et}_{2} \mathrm{O}$ solution (1M, $2.5 \mathrm{~mL}, 2.5 \mathrm{mmol}, 5.0$ equiv) was added. Subsequently, the nucleophile (2.5 mmol, 5.0 equiv) was added and the resulting mixture was stirred for 16 hours at room temperature. The crude mixture was acidified to $\mathrm{pH} 1$ with $1 \mathrm{M} \mathrm{HCl}$ and extracted with $\mathrm{CH}_{2} \mathrm{Cl}_{2}$. The organic layer was washed with $1 \mathrm{M} \mathrm{HCl}$ and brine, dried over $\mathrm{Na}_{2} \mathrm{SO}_{4}$ and concentrated under reduced pressure to yield the corresponding products $\mathbf{1 0 .}$ 


\section{Butyl $N$-acetyl- $N$-benzylleucinate (10e).}

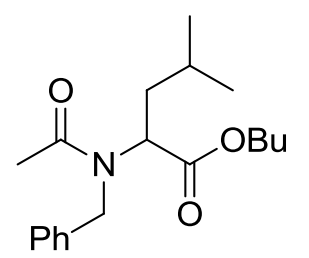

Following general procedure IX with butanol $(229 \mu \mathrm{L}, 2.5 \mathrm{mmol}, 5.0$ equiv) the corresponding ester was obtained as a yellow oil (160 mg, $0.5 \mathrm{mmol}, 100 \%)$. Two rotamers were present on NMR timescale $\left(\mathrm{R}^{1}: \mathrm{R}^{2}=1: 0.4\right) .{ }^{1} \mathrm{H} \mathrm{NMR}\left(\mathrm{CDCl}_{3}, 500\right.$ MHz) $\delta 7.34-7.12(\mathrm{~m}, 7 \mathrm{H}), 4.80(\mathrm{t}, J=6.6 \mathrm{~Hz}, 1 \mathrm{H}), 4.65(\mathrm{~m}, 1.4 \mathrm{H}), 4.43(\mathrm{~m}, 1.4 \mathrm{H})$, $4.37(\mathrm{~m}, 0.4 \mathrm{H}), 4.00(\mathrm{~m}, 1 \mathrm{H}), 3.93(\mathrm{~m}, 1.4 \mathrm{H}), 3.76(\mathrm{~m}, 0.4 \mathrm{H}), 2.23(\mathrm{~s}, 1.2 \mathrm{H}), 2.06(\mathrm{~s}$, $3 \mathrm{H}), 1.82-1.75(\mathrm{~m}, 1.4 \mathrm{H}), 1.56-1.43(\mathrm{~m}, 5.6 \mathrm{H}), 1.33-1.24(\mathrm{~m}, 2.8 \mathrm{H}), 0.89-0.83(\mathrm{~m}, 8.4 \mathrm{H}), 0.73(\mathrm{~d}, J=6.5$ $\mathrm{Hz}, 3 \mathrm{H}), 0.65(\mathrm{~d}, J=6.7 \mathrm{~Hz}, 1.2 \mathrm{H}) .{ }^{13} \mathrm{C} \mathrm{NMR}\left(\mathrm{CDCl}_{3}, 125 \mathrm{MHz}\right) \delta 171.8\left(\mathrm{C}_{\mathrm{q}}\right), 171.7\left(\mathrm{C}_{\mathrm{q}}\right), 171.5$ $\left(\mathrm{C}_{\mathrm{q}}\right), 170.8\left(\mathrm{C}_{\mathrm{q}}\right), 137.9\left(\mathrm{C}_{\mathrm{q}}\right), 137.0\left(\mathrm{C}_{\mathrm{q}}\right), 128.5(\mathrm{CH}), 128.0(\mathrm{CH}), 127.8(\mathrm{CH}), 127.3(\mathrm{CH}), 126.8(\mathrm{CH})$, $126.3(\mathrm{CH}), 65.1\left(\mathrm{CH}_{2}\right), 64.7\left(\mathrm{CH}_{2}\right), 58.9(\mathrm{CH}), 56.00\left(\mathrm{CH}_{2}\right), 50.6\left(\mathrm{CH}_{2}\right), 46.3\left(\mathrm{CH}_{2}\right), 38.3\left(\mathrm{CH}_{2}\right), 38.1$ $\left(\mathrm{CH}_{2}\right), 30.3\left(\mathrm{CH}_{2}\right), 30.1\left(\mathrm{CH}_{2}\right), 25.0(\mathrm{CH}), 24.3(\mathrm{CH}), 22.2\left(\mathrm{CH}_{3}\right), 22.1\left(\mathrm{CH}_{3}\right), 22.0\left(\mathrm{CH}_{3}\right), 21.9\left(\mathrm{CH}_{3}\right)$, $21.8\left(\mathrm{CH}_{3}\right), 18.9\left(\mathrm{CH}_{2}\right), 18.9\left(\mathrm{CH}_{2}\right), 13.5\left(\mathrm{CH}_{3}\right), 13.5\left(\mathrm{CH}_{3}\right)$. IR (neat): vmax $\left(\mathrm{cm}^{-1}\right)=2957(\mathrm{~m}), 1734(\mathrm{~s})$, 1653 (s), 633 (s), 536 (s), 498 (s). HRMS (ESI): $m / z$ calculated for $\mathrm{C}_{19} \mathrm{H}_{30} \mathrm{NO}_{3}[\mathrm{M}+\mathrm{H}]^{+} 320.2220$, found 320.2206 .

\section{Isopropyl $N$-acetyl- $N$-benzylleucinate (10f).}

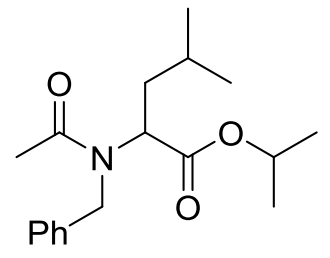

Following general procedure IX with 2-propanol (190 $\mu \mathrm{L}, 2.5 \mathrm{mmol}, 5.0$ equiv) the corresponding ester was obtained as a slightly yellow oil $(153 \mathrm{mg}, 0.5 \mathrm{mmol}$, $100 \%)$. Two rotamers were present on NMR timescale $\left(\mathrm{R}^{1}: \mathrm{R}^{2}=1: 0.4\right) .{ }^{1} \mathrm{H}$ NMR $\left(\mathrm{CDCl}_{3}, 500 \mathrm{MHz}\right) \delta 7.40-7.15(\mathrm{~m}, 7 \mathrm{H}), 4.94(\mathrm{qq}, J=6.3,6.3 \mathrm{~Hz}, 1 \mathrm{H}), 5.89-5.80$ $(\mathrm{m}, 1.8 \mathrm{H}), 4.70(\mathrm{~d}, J=17.5 \mathrm{~Hz}, 1 \mathrm{H}), 4.47(\mathrm{~d}, J=17.5 \mathrm{~Hz}, 1 \mathrm{H}), 4.33(\mathrm{t}, J=6.3 \mathrm{~Hz}$, $0.4 \mathrm{H}), 4.28(\mathrm{~d}, J=15.5 \mathrm{~Hz}, 0.4 \mathrm{H}), 2.56(\mathrm{~s}, 1,2 \mathrm{H}), 2.09(\mathrm{~s}, 3 \mathrm{H}), 1.84(\mathrm{~m}, 1 \mathrm{H}), 1.73$ $(\mathrm{m}, 0.4 \mathrm{H}), 1.64-1.35(\mathrm{~m}, 2.8 \mathrm{H}), 1.21(\mathrm{~d}, J=6.0 \mathrm{~Hz}, 6 \mathrm{H}), 1.19(\mathrm{~d}, J=6.0 \mathrm{~Hz}, 1.2 \mathrm{H}), 1.13(\mathrm{~d}, J=6.0 \mathrm{~Hz}$, $1.2 \mathrm{H}), 0.88(\mathrm{~m}, 4.2 \mathrm{H}), 0.78(\mathrm{~d}, J=6.5 \mathrm{~Hz}, 3 \mathrm{H}), 0.67(\mathrm{~d}, J=6.5 \mathrm{~Hz}, 1.2 \mathrm{H}) .{ }^{13} \mathrm{C} \mathrm{NMR}\left(\mathrm{CDCl}_{3}, 125 \mathrm{MHz}\right) \delta$ $172.0\left(\mathrm{C}_{\mathrm{q}}\right), 171.9\left(\mathrm{C}_{\mathrm{q}}\right), 171.2\left(\mathrm{C}_{\mathrm{q}}\right), 170.5\left(\mathrm{C}_{\mathrm{q}}\right), 138.4\left(\mathrm{C}_{\mathrm{q}}\right), 137.4\left(\mathrm{C}_{\mathrm{q}}\right), 128.7(\mathrm{CH}), 128.2(\mathrm{CH}), 127.9$ $(\mathrm{CH}), 127.4(\mathrm{CH}), 126.9(\mathrm{CH}), 126.5(\mathrm{CH}), 69.3(\mathrm{CH}), 68.6(\mathrm{CH}), 59.5(\mathrm{CH}), 56.5(\mathrm{CH}), 50.8\left(\mathrm{CH}_{2}\right)$, $46.8\left(\mathrm{CH}_{2}\right), 38.6\left(\mathrm{CH}_{2}\right), 25.3(\mathrm{CH}), 24.5(\mathrm{CH}), 22.5\left(\mathrm{CH}_{3}\right), 22.4\left(\mathrm{CH}_{3}\right), 22.3\left(\mathrm{CH}_{3}\right), 22.3\left(\mathrm{CH}_{3}\right), 22.2$ $\left(\mathrm{CH}_{3}\right), 22.0\left(\mathrm{CH}_{3}\right), 21.7\left(\mathrm{CH}_{3}\right), 21.6\left(\mathrm{CH}_{3}\right), 21.5\left(\mathrm{CH}_{3}\right)$. IR (neat): vmax $\left(\mathrm{cm}^{-1}\right)=2957(\mathrm{~m}), 1730(\mathrm{~s}), 1653$ (s), 1410 (m), 1177 (m), 1105 (s), 968 (m), 727 (s), 698 (s). HRMS (ESI): m/z calculated for $\mathrm{C}_{18} \mathrm{H}_{28} \mathrm{NO}_{3}$ $[\mathrm{M}+\mathrm{H}]^{+}$306.2064, found 306.2051.

\section{But-3-yn-1-yl $N$-acetyl- $N$-benzylleucinate $(10 \mathrm{~g})$.}

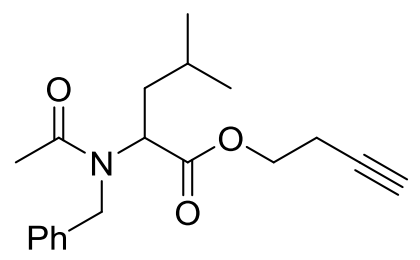

Following general procedure IX with 3-butyn-1-ol $(75 \mu \mathrm{L}, 1.0 \mathrm{mmol}, 2.0$ equiv) the corresponding ester was obtained after purification by flash chromatography (cyclohexane $:$ EtOAc $=4: 1)$ as a colorless oil $(102 \mathrm{mg}$, $0.32 \mathrm{mmol}, 65 \%)$. Two rotamers were present on NMR timescale $\left(\mathrm{R}^{1}: \mathrm{R}^{2}=\right.$ $1: 0.35) .{ }^{1} \mathrm{H} \mathrm{NMR}\left(\mathrm{CDCl}_{3}, 500 \mathrm{MHz}\right) \delta 7.42-7.18(\mathrm{~m}, 6.75 \mathrm{H}), 4.77(\mathrm{t}, J=$ $7.0 \mathrm{~Hz}, 1 \mathrm{H}), 4.75(\mathrm{~m}, 1.35 \mathrm{H}), 4.50(\mathrm{~m}, 1.35 \mathrm{H}), 4.41(\mathrm{t}, J=7.0 \mathrm{~Hz}, 0.35 \mathrm{H})$, $4.13(\mathrm{~m}, 2 \mathrm{H}), 4.03(\mathrm{~m}, 0.35 \mathrm{H}), 3.87(\mathrm{~m}, 0.35 \mathrm{H}), 2.48(\mathrm{~m}, 2 \mathrm{H}), 2.39(\mathrm{~m}, 0.70 \mathrm{H}), 2.29(\mathrm{~s}, 1 \mathrm{H}), 2.12(\mathrm{~s}, 3 \mathrm{H})$, $1.99(\mathrm{~s}, 1.05 \mathrm{H}), 1.98(0.35 \mathrm{H}), 1.89(\mathrm{~m}, 1 \mathrm{H}), 1.78(\mathrm{~m}, 0.35 \mathrm{H}), 1.71-1.38(\mathrm{~m}, 2.7 \mathrm{H}), 0.89(\mathrm{~d}, J=6.5 \mathrm{~Hz}$ ,4.05H), $0.78(\mathrm{~d}, J=6.5 \mathrm{~Hz}, 3 \mathrm{H}), 0.73(\mathrm{~d}, J=6.5 \mathrm{~Hz}, 1.05 \mathrm{H}) .{ }^{13} \mathrm{C} \mathrm{NMR}\left(\mathrm{CDCl}_{3}, 125 \mathrm{MHz}\right) \delta 171.9\left(\mathrm{C}_{\mathrm{q}}\right)$, $171.8\left(\mathrm{C}_{\mathrm{q}}\right), 171.4\left(\mathrm{C}_{\mathrm{q}}\right), 170.7\left(\mathrm{C}_{\mathrm{q}}\right), 138.1\left(\mathrm{C}_{\mathrm{q}}\right), 137.1\left(\mathrm{C}_{\mathrm{q}}\right), 128.7(\mathrm{CH}), 128.2(\mathrm{CH}), 128.1(\mathrm{CH}), 127.6$ $(\mathrm{CH}), 127.0(\mathrm{CH}), 126.6(\mathrm{CH}), 80.1\left(\mathrm{C}_{\mathrm{q}}\right), 70.2(\mathrm{CH}), 69.8(\mathrm{CH}), 62.8\left(\mathrm{CH}_{2}\right), 62.6\left(\mathrm{CH}_{2}\right), 58.9(\mathrm{CH}), 56.2$ $(\mathrm{CH}), 51.1\left(\mathrm{CH}_{2}\right), 46.3\left(\mathrm{CH}_{2}\right), 38.4\left(\mathrm{CH}_{2}\right), 38.2\left(\mathrm{CH}_{2}\right), 25.2(\mathrm{CH}), 24.4(\mathrm{CH}), 22.5\left(\mathrm{CH}_{3}\right), 22.4\left(\mathrm{CH}_{3}\right)$, 
$22.4\left(\mathrm{CH}_{3}\right), 22.3\left(\mathrm{CH}_{3}\right), 22.2\left(\mathrm{CH}_{3}\right), 22.0\left(\mathrm{CH}_{3}\right), 18.8\left(\mathrm{CH}_{2}\right), 18.7\left(\mathrm{CH}_{2}\right)$. IR (neat): vmax $\left(\mathrm{cm}^{-1}\right)=3279$ (m), 2957 (m), 1736 (s), 1647 (s), 1410 (m), 1240 (m), 1171 (m), 729 (s), 698 (s), 638 (m), HRMS (ESI): $\mathrm{m} / \mathrm{z}$ calculated for $\mathrm{C}_{19} \mathrm{H}_{26} \mathrm{NO}_{3}[\mathrm{M}+\mathrm{H}]^{+} 316.1907$, found 316.1892 .

\section{$S$-Ethyl 2-( $N$-benzylacetamido)-4-methylpentanethioate (10h).}<smiles>CCSC(=O)C(CC(C)C)N(Cc1ccccc1)C(C)=O</smiles>

Following general procedure IX with ethanethiol (cosolvent), the corresponding thioester was obtained after purification by flash chromatography (cyclohexane : EtOAc $=6: 1)$ as a yellow oil $(132 \mathrm{mg}, 0.43 \mathrm{mmol}, 86 \%)$. Two rotamers were present on NMR timescale $\left(\mathrm{R}^{1}: \mathrm{R}^{2}=1: 0.4\right) .{ }^{1} \mathrm{H} \mathrm{NMR}\left(\mathrm{CDCl}_{3}, 500 \mathrm{MHz}\right) \delta 7.42-7.18(\mathrm{~m}$, $7 \mathrm{H}), 5.30(\mathrm{t}, J=7.0 \mathrm{~Hz}, 1 \mathrm{H}), 5.10(\mathrm{~d}, J=15.5 \mathrm{~Hz}, 0.4 \mathrm{H}), 4.70(\mathrm{~d}, J=17.5 \mathrm{~Hz}, 1 \mathrm{H})$, $4.44(\mathrm{~d}, J=17.5 \mathrm{~Hz}, 1 \mathrm{H}), 4.40(\mathrm{t}, J=7.0 \mathrm{~Hz}, 0.4 \mathrm{H}), 4.02(\mathrm{~d}, J=15.5 \mathrm{~Hz}, 0.4 \mathrm{H}), 2.85$ $(\mathrm{m}, 2.8 \mathrm{H}), 2.29(\mathrm{~s}, 1.2 \mathrm{H}), 2.13(\mathrm{~s}, 3 \mathrm{H}), 1.80(\mathrm{~m}, 1.4 \mathrm{H}), 1.54(\mathrm{~m}, 1.4 \mathrm{H}), 1.43(\mathrm{~m}, 1 \mathrm{H}), 1.3(\mathrm{~m}, 0.4 \mathrm{H}), 1.23$ $(\mathrm{m}, 4.2 \mathrm{H}), 0.88(\mathrm{~d}, J=6.5 \mathrm{~Hz}, 3 \mathrm{H}), 0.84(\mathrm{~d}, J=6.5 \mathrm{~Hz}, 1.2 \mathrm{H}), 0.74(\mathrm{~d}, J=6.5 \mathrm{~Hz}, 3 \mathrm{H}), 0.53(\mathrm{~d}, J=6.5$ $\mathrm{Hz}, 1.2 \mathrm{H}) .{ }^{13} \mathrm{C}$ NMR $\left(\mathrm{CDCl}_{3}, 125 \mathrm{MHz}\right) \delta 199.4\left(\mathrm{C}_{\mathrm{q}}\right), 199.2\left(\mathrm{C}_{\mathrm{q}}\right), 172.4\left(\mathrm{C}_{\mathrm{q}}\right), 171.9\left(\mathrm{C}_{\mathrm{q}}\right), 138.5\left(\mathrm{C}_{\mathrm{q}}\right)$, $137.3\left(\mathrm{C}_{\mathrm{q}}\right), 128.7(\mathrm{CH}), 128.3(\mathrm{CH}), 127.8(\mathrm{CH}), 127.4(\mathrm{CH}), 127.0(\mathrm{CH}), 126.2(\mathrm{CH}), 66.9(\mathrm{CH}), 62.1$ $(\mathrm{CH}), 50.2\left(\mathrm{CH}_{2}\right), 47.7\left(\mathrm{CH}_{2}\right), 38.3\left(\mathrm{CH}_{2}\right), 37.8\left(\mathrm{CH}_{2}\right), 25.3(\mathrm{CH}), 24.5(\mathrm{CH}), 23.6\left(\mathrm{CH}_{2}\right), 23.4\left(\mathrm{CH}_{2}\right)$, $22.4\left(\mathrm{CH}_{3}\right), 22.4\left(\mathrm{CH}_{3}\right), 22.3\left(\mathrm{CH}_{3}\right), 21.8\left(\mathrm{CH}_{3}\right), 14.4\left(\mathrm{CH}_{3}\right)$. IR (neat): vmax $\left(\mathrm{cm}^{-1}\right)=2957(\mathrm{~m}), 1681(\mathrm{~m})$, 1655 (s), 1402 (s), 972 (m), 727 (s), 698 (s). HRMS (ESI): $m / z$ calculated for $\mathrm{C}_{17} \mathrm{H}_{26} \mathrm{NO}_{2} \mathrm{~S}[\mathrm{M}+\mathrm{H}]^{+}$ 308.1679 , found 308.1659 .

\section{$N$-(6-bromopyridin-2-yl)-1-phenethyl-4-(N-phenylpropionamido)piperidine-4-carboxamide (14).}

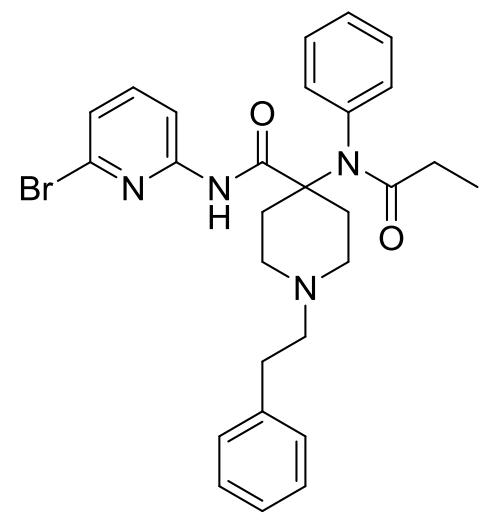

Aniline (182 $\mu \mathrm{L}, 2.0 \mathrm{mmol}, 1$ equiv) and 1-phenethyl-4-piperidone (13, $407 \mathrm{mg}, 2.0 \mathrm{mmol}, 1$ equiv) were dissolved in TFE (5 mL). Then, $3 \AA$ MS $(500 \mathrm{mg})$ were added and the resulting mixture was stirred overnight at room temperature before adding propionic acid $(299 \mu \mathrm{L}, 4.0 \mathrm{mmol}, 2$ equiv). Lastly, after one hour, 2-bromo-6-isocyanopyridine $(\mathbf{6 1}, 439 \mathrm{mg}$, 2.4 mmol, 1.2 equiv) was added and the mixture stirred for 24 hours. The reaction mixture was filtered and washed with EtOAc. The filtrate was washed with sat. $\mathrm{NaHCO}_{3}(2 \times)$ and with Brine, dried over $\mathrm{Na}_{2} \mathrm{SO}_{4}$, filtered and concentrated under reduced pressure. The crude product was purified with flash chromatography (cyclohexane : EtOAc $=1: 1)$ to yield an off-white solid (982 mg, $1.83 \mathrm{mmol}, 92 \%)$. m.p.: $151{ }^{\circ} \mathrm{C} .{ }^{1} \mathrm{H}$ NMR $\left(\mathrm{CDCl}_{3}, 500 \mathrm{MHz}\right) \delta 8.82(\mathrm{bs}, 1 \mathrm{H}), 8.25(\mathrm{~d}, J=8.0 \mathrm{~Hz}, 1 \mathrm{H}), 7.52(\mathrm{t}, J=8.0 \mathrm{~Hz}, 1 \mathrm{H}), 7.55(\mathrm{~m}, 3 \mathrm{H}), 7.33$ $(\mathrm{d}, J=6.0 \mathrm{~Hz}, 2 \mathrm{H}), 7.28-7.10(\mathrm{~m}, 6 \mathrm{H}), 2.84-2.69(\mathrm{~m}, 4 \mathrm{H}), 2.60-2.40(\mathrm{~m}, 6 \mathrm{H}), 1.92(\mathrm{q}, J=7.5 \mathrm{~Hz}, 2 \mathrm{H})$, $1.81(\mathrm{~m}, 2 \mathrm{H}), 0.95(\mathrm{t}, J=7.5 \mathrm{~Hz}, 3 \mathrm{H}) .{ }^{13} \mathrm{C} \mathrm{NMR}\left(\mathrm{CDCl}_{3}, 125 \mathrm{MHz}\right) \delta 175.2\left(\mathrm{C}_{\mathrm{q}}\right), 171.6\left(\mathrm{C}_{\mathrm{q}}\right), 151.6\left(\mathrm{C}_{\mathrm{q}}\right)$, $140.3(\mathrm{CH}), 140.0\left(\mathrm{C}_{\mathrm{q}}\right), 139.2\left(\mathrm{C}_{\mathrm{q}}\right), 139.0\left(\mathrm{C}_{\mathrm{q}}\right), 130.5(\mathrm{CH}), 129.5(\mathrm{CH}), 128.8(\mathrm{CH}), 128.5(\mathrm{CH}), 128.3$ $(\mathrm{CH}), 125.9(\mathrm{CH}), 123.3(\mathrm{CH}), 112.3(\mathrm{CH}), 64.4\left(\mathrm{C}_{\mathrm{q}}\right), 60.1\left(\mathrm{CH}_{2}\right), 50.0\left(\mathrm{CH}_{2}\right), 33.6\left(\mathrm{CH}_{2}\right), 33.5\left(\mathrm{CH}_{2}\right)$, $29.6\left(\mathrm{CH}_{2}\right), 9.1\left(\mathrm{CH}_{3}\right)$. IR (neat): vmax $\left(\mathrm{cm}^{-1}\right)=1701(\mathrm{~s}), 1649(\mathrm{~s}), 1562(\mathrm{~s}), 1512(\mathrm{~s}), 1429(\mathrm{~s}), 1381(\mathrm{~s})$, 1286 (m), 1254 (m), 1126 (s), 727 (s), 700 (s), 575 (m). HRMS (ESI): m/z calculated for $\mathrm{C}_{28} \mathrm{H}_{32} \mathrm{BrN}_{4} \mathrm{O}_{2}$ $[\mathrm{M}+\mathrm{H}]^{+}$535.1703, found 535.1699. 


\section{Carfentanil (15).}

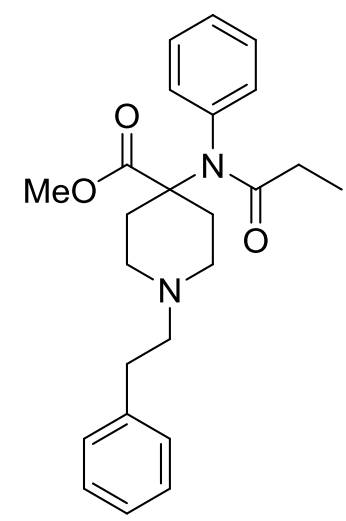

Acetyl chloride $(89 \mu \mathrm{L}, 1.25 \mathrm{mmol}, 5$ equiv) was added to a solution of $\mathrm{N}$-(6bromopyridin-2-yl)-1-phenylethyl-4-( $N$-phenylpropionamido)-piperidine-4carboxamide (14, $134 \mathrm{mg}, 0.25 \mathrm{mmol}, 1$ equiv) in $\mathrm{MeOH}(3 \mathrm{~mL})$. The resulting mixture stirred overnight at $45{ }^{\circ} \mathrm{C}$. Then, saturated aqueous $\mathrm{NaHCO}_{3}$ and $\mathrm{EtOAc}$ were added. The layers were separated and the water layer was extracted with EtOAc $(3 \times)$. The organic layers were combined and concentrated under reduced pressure. The crude product was purified with flash chromatography (cyclohexane : EtOAc $=1: 1)$ to yield a slightly yellow oil $(97 \mathrm{mg}, 0.246 \mathrm{mmol}$, 98\%). ${ }^{1} \mathrm{H} \mathrm{NMR}\left(\mathrm{CDCl}_{3}, 500 \mathrm{MHz}\right) \delta 7.39(\mathrm{~m}, 3 \mathrm{H}), 7.32(\mathrm{~d}, J=7.5 \mathrm{~Hz}, 2 \mathrm{H}), 7.23$ $(\mathrm{t}, J=7.5 \mathrm{~Hz}, 2 \mathrm{H}), 7.14(\mathrm{~m}, 3 \mathrm{H}), 3.79(\mathrm{~s}, 3 \mathrm{H}), 2.71(\mathrm{~m}, 4 \mathrm{H}), 2.53(\mathrm{~m}, 2 \mathrm{H}), 2.44$ $(\mathrm{t}, J=11.5 \mathrm{~Hz}, 2 \mathrm{H}), 2.31(\mathrm{~d}, J=13.0 \mathrm{~Hz}, 2 \mathrm{H}), 1.87(\mathrm{q}, J=7.5 \mathrm{~Hz}, 2 \mathrm{H}), 1.86(\mathrm{t}, J$ $=12.0 \mathrm{~Hz}, 2 \mathrm{H}), 0.94(\mathrm{t}, J=7.5 \mathrm{~Hz}, 3 \mathrm{H}) .{ }^{13} \mathrm{C} \mathrm{NMR}\left(\mathrm{CDCl}_{3}, 125 \mathrm{MHz}\right) \delta 174.0\left(\mathrm{C}_{\mathrm{q}}\right), 173.8\left(\mathrm{C}_{\mathrm{q}}\right), 140.1$ $\left(\mathrm{C}_{\mathrm{q}}\right), 139.2\left(\mathrm{C}_{\mathrm{q}}\right), 130.5(\mathrm{CH}), 129.2(\mathrm{CH}), 128.6(\mathrm{CH}), 128.5(\mathrm{CH}), 128.2(\mathrm{CH}), 125.9(\mathrm{CH}), 62.7\left(\mathrm{C}_{\mathrm{q}}\right)$, $60.3\left(\mathrm{CH}_{2}\right), 52.0\left(\mathrm{CH}_{3}\right), 49.8\left(\mathrm{CH}_{2}\right), 33.6\left(\mathrm{CH}_{2}\right), 33.5\left(\mathrm{CH}_{2}\right), 28.9\left(\mathrm{CH}_{2}\right), 9.0\left(\mathrm{CH}_{3}\right)$. HRMS (ESI): $\mathrm{m} / z$ calculated for $\mathrm{C}_{24} \mathrm{H}_{31} \mathrm{~N}_{2} \mathrm{O}_{3}[\mathrm{M}+\mathrm{H}]^{+}$395.2329, found 395.2324. 


\section{Copies of NMR spectra}

Isocyanides (6):

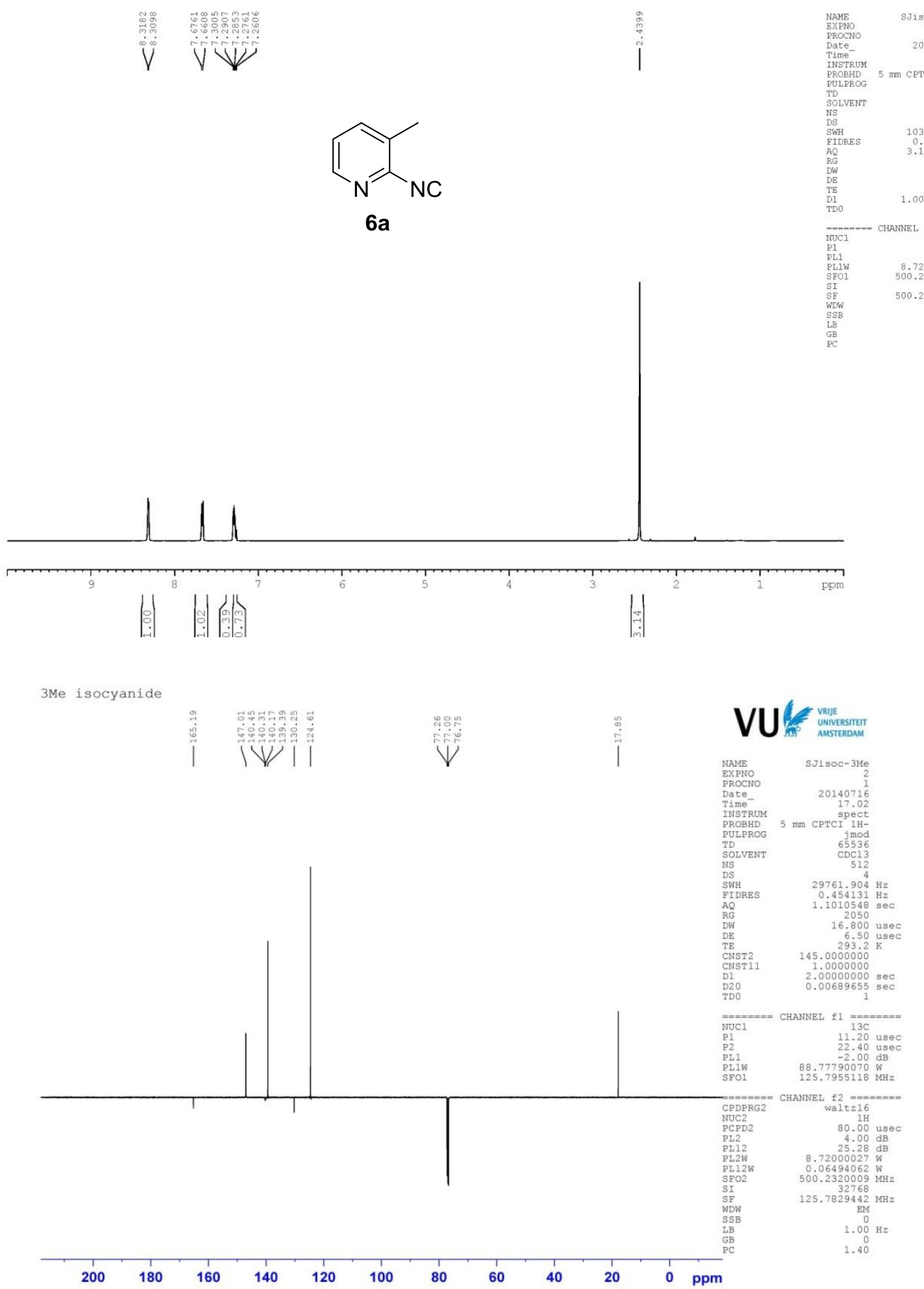




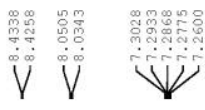

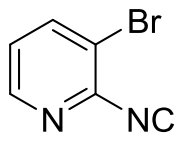

$6 b$
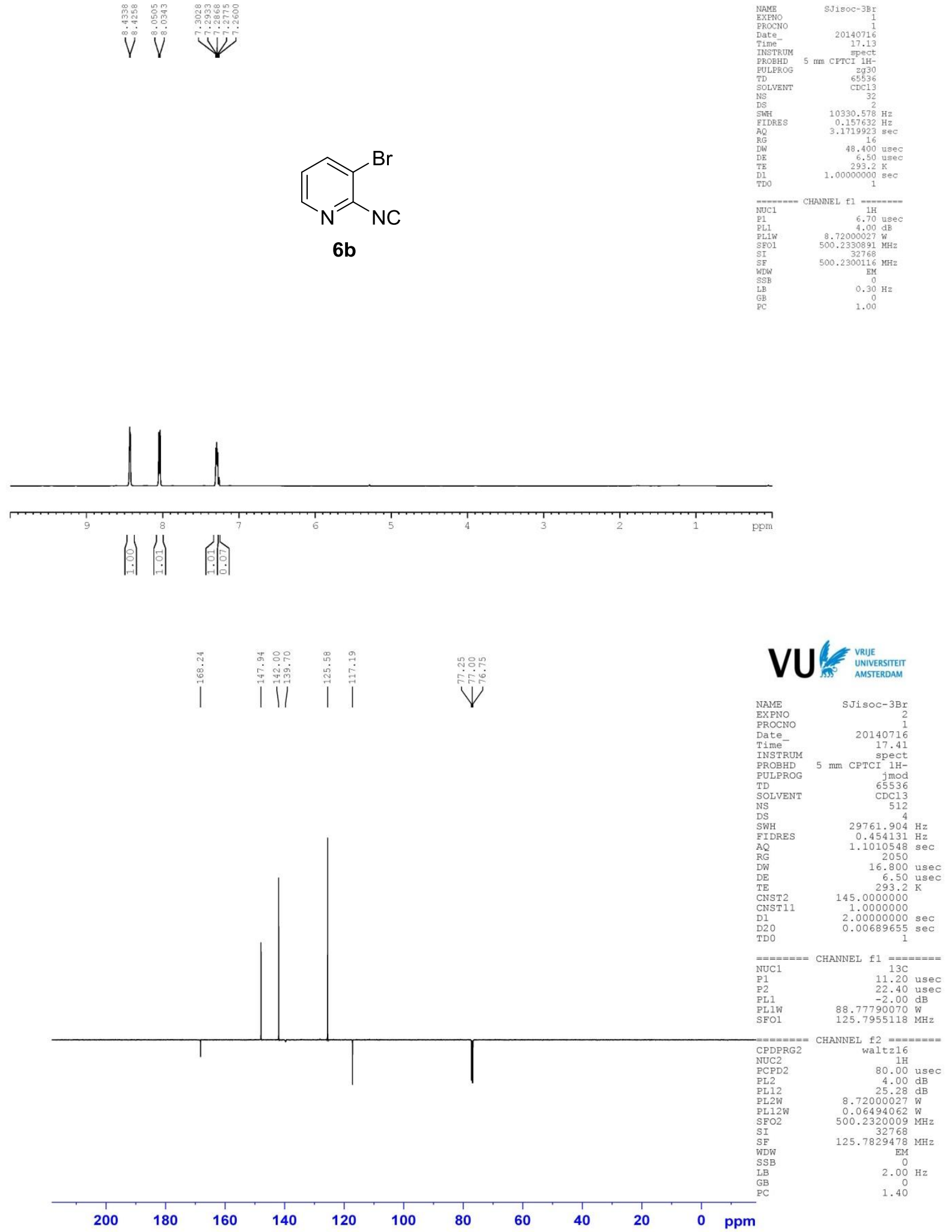


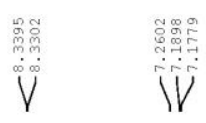
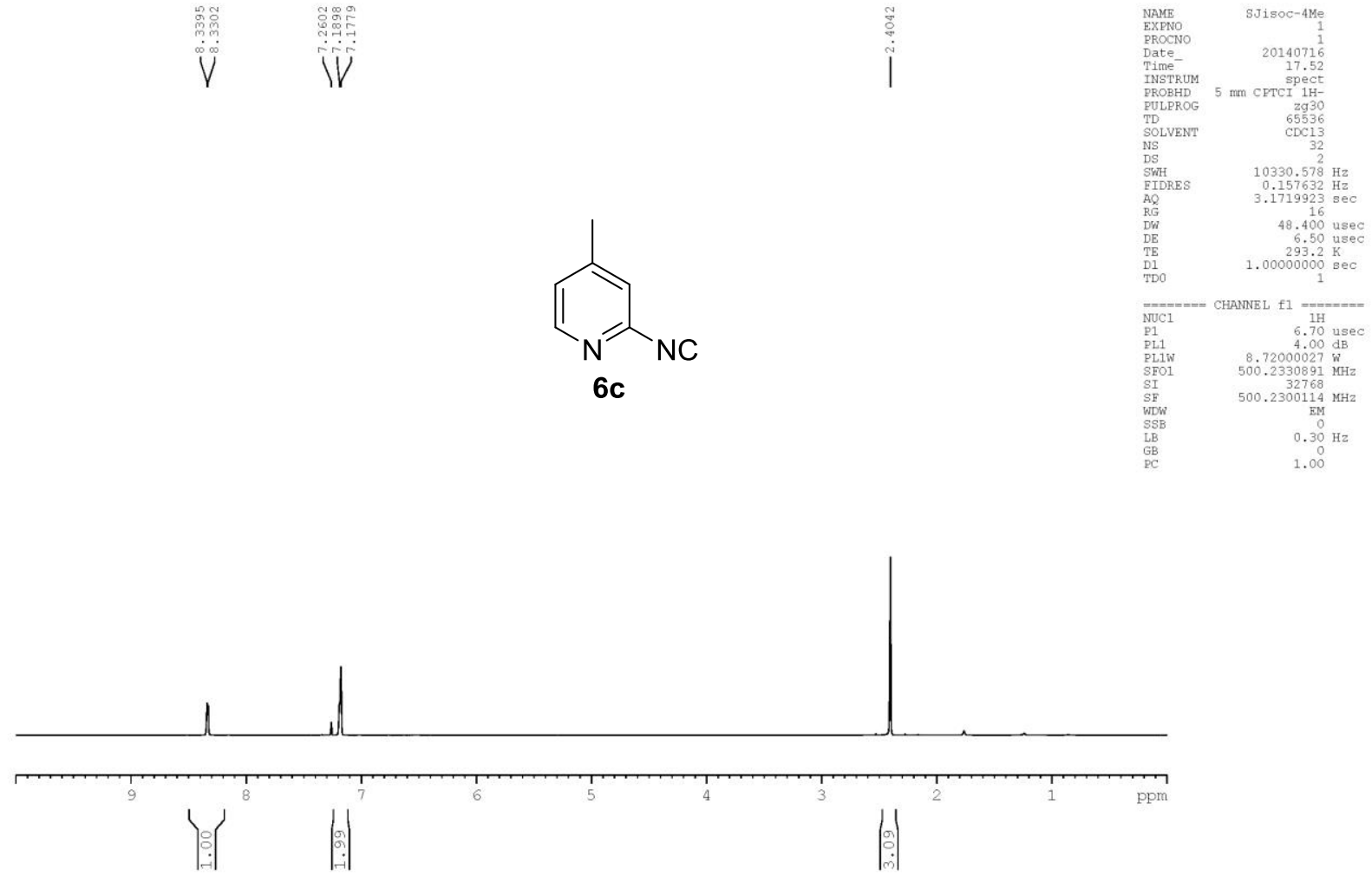

4Me isocyanide
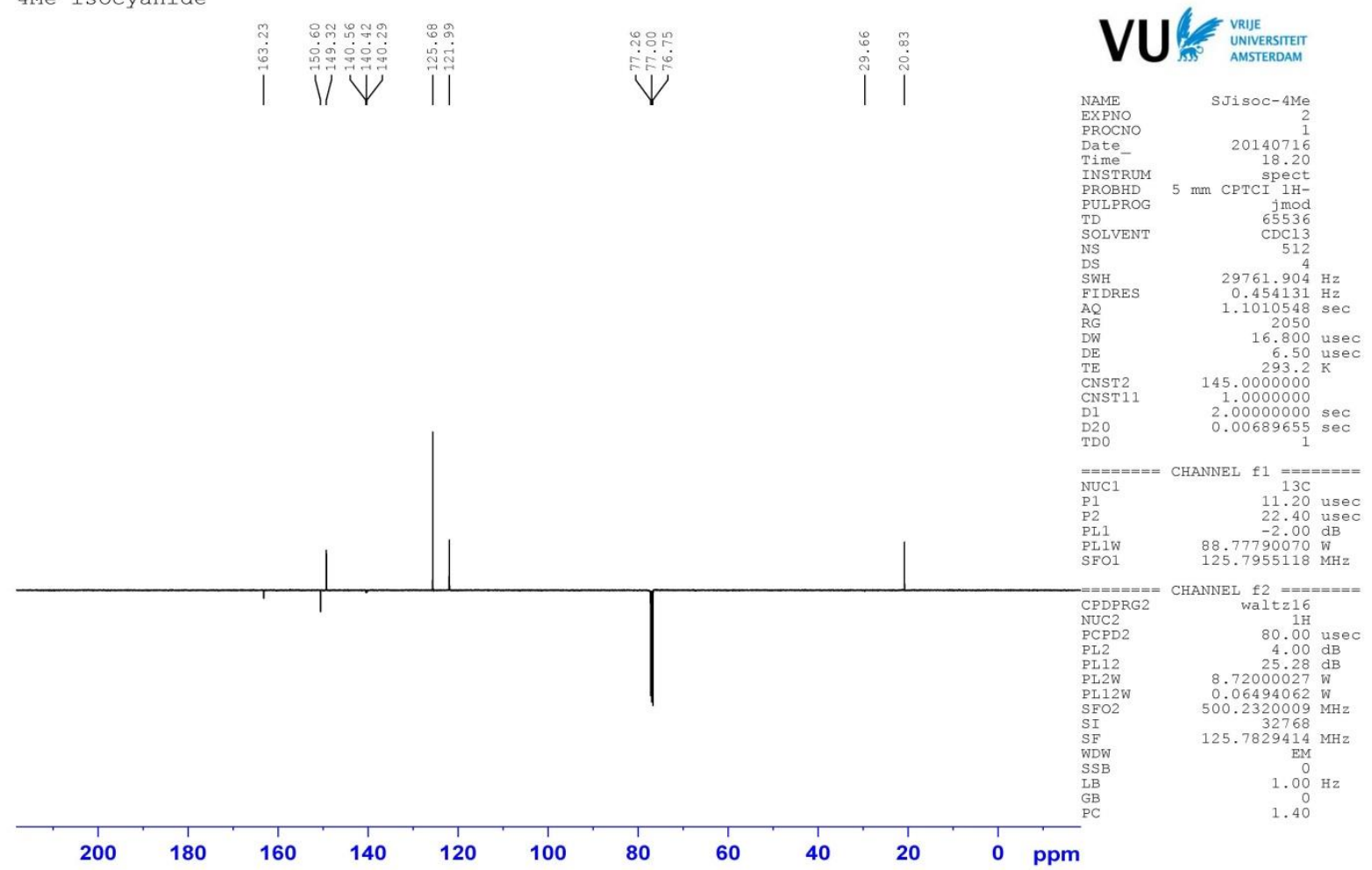

S31 
Vै
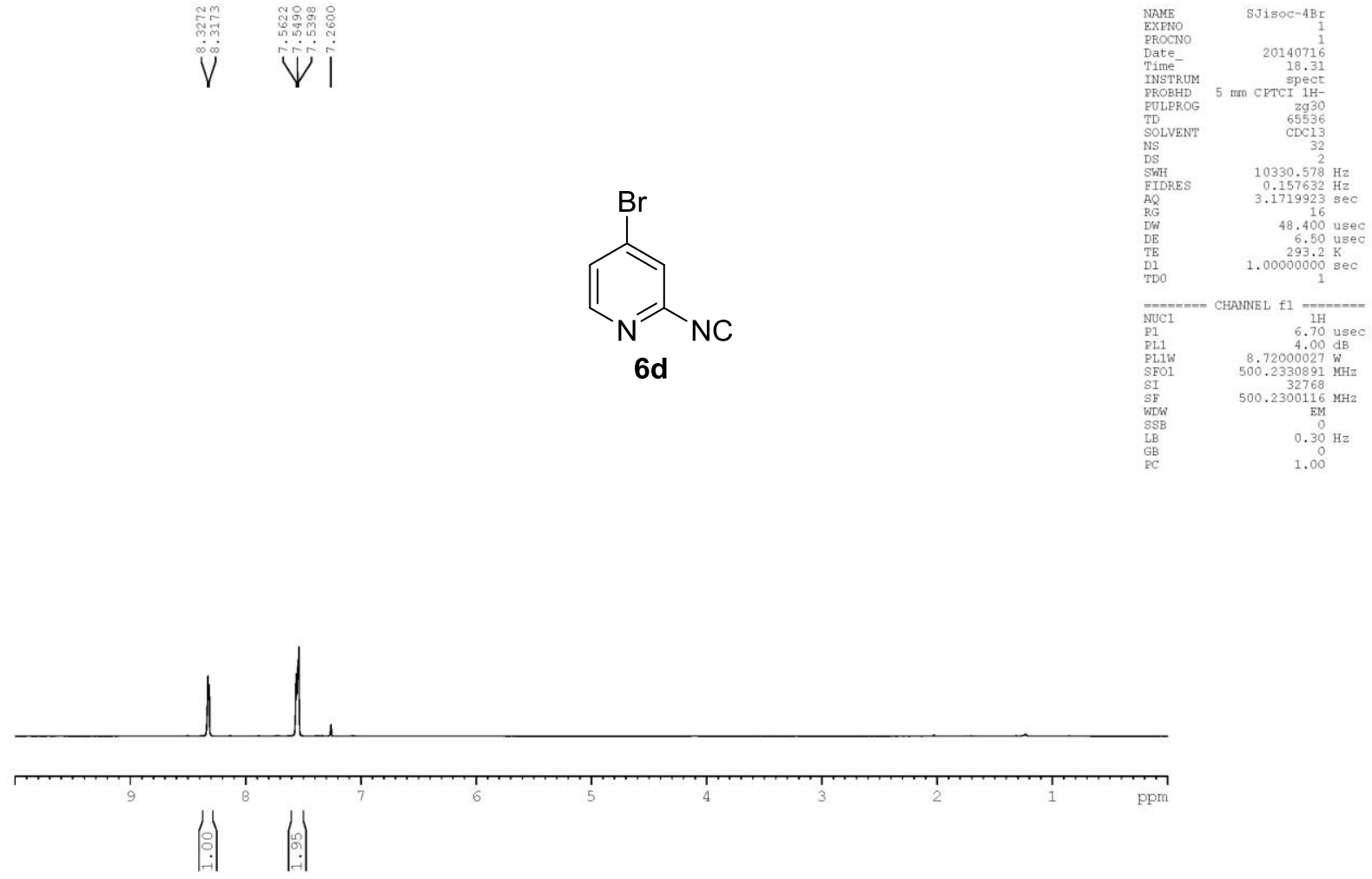

$4 \mathrm{Br}$ isocyanide
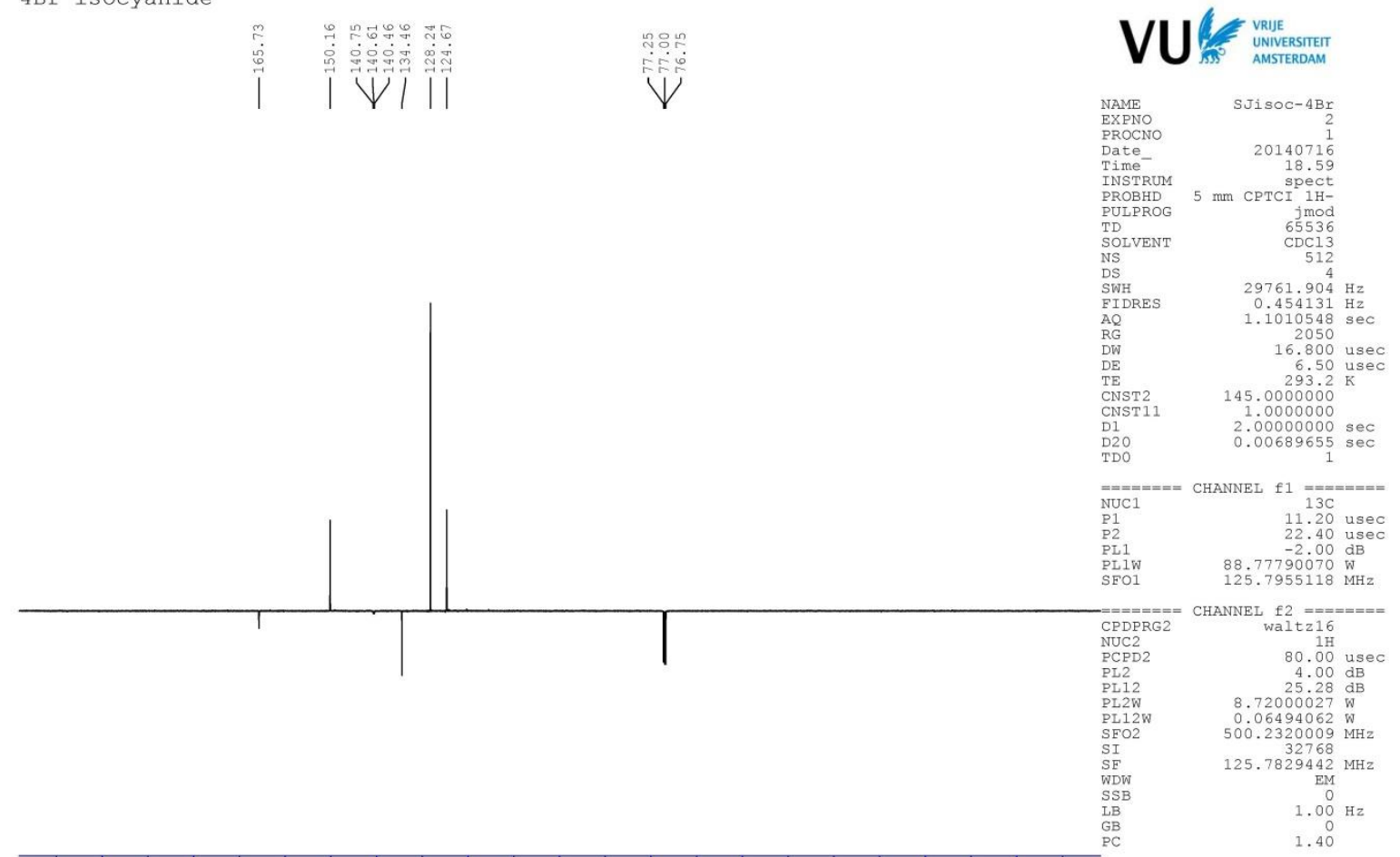

1.40

$\begin{array}{llllllllllll}200 & 180 & 160 & 140 & 120 & 100 & 80 & 60 & 40 & 20 & 0 & \text { ppm }\end{array}$

S32 

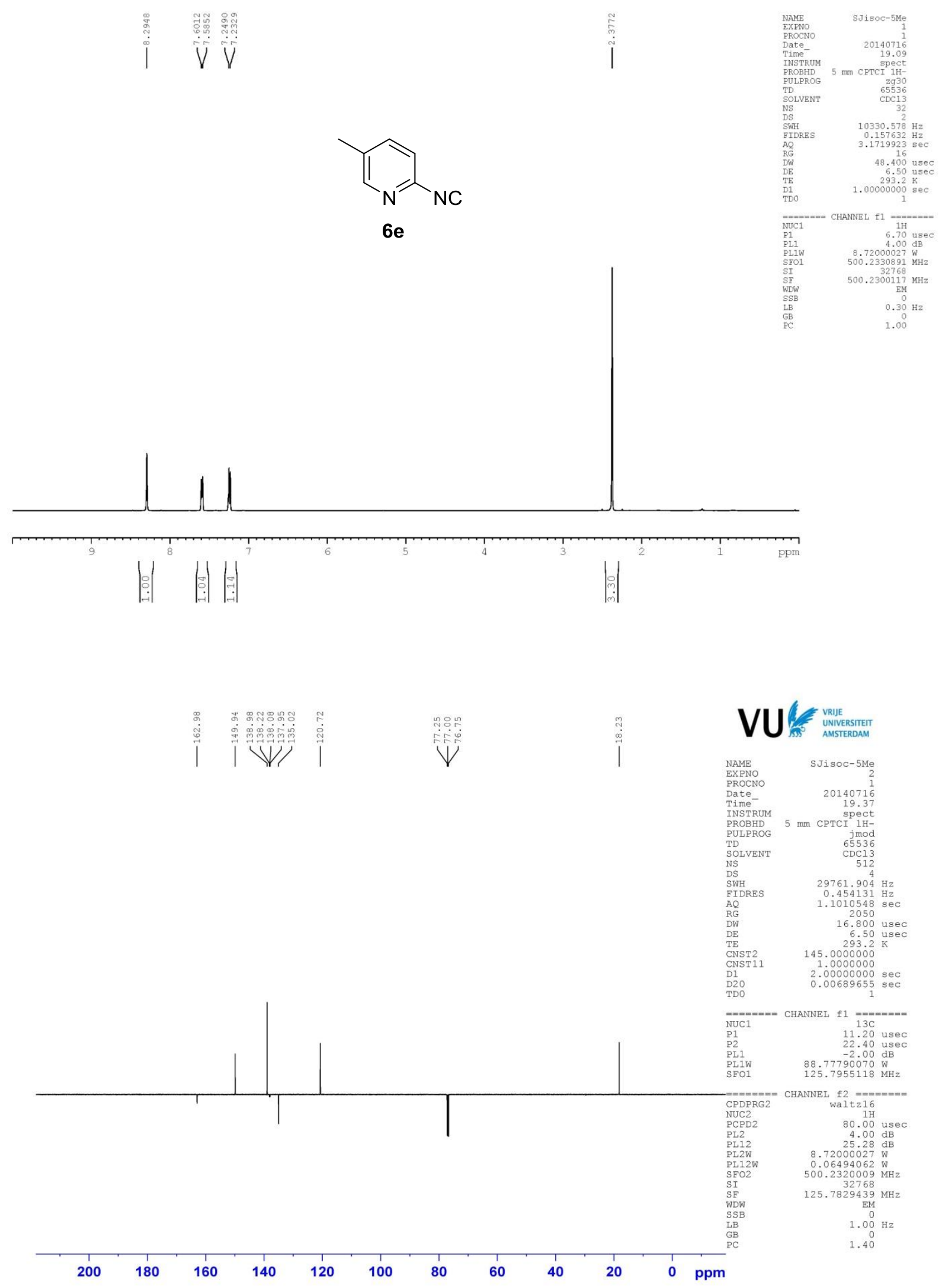

S33 

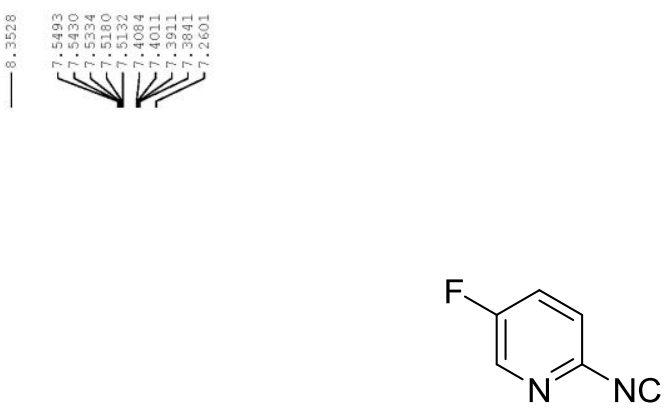

$6 f$

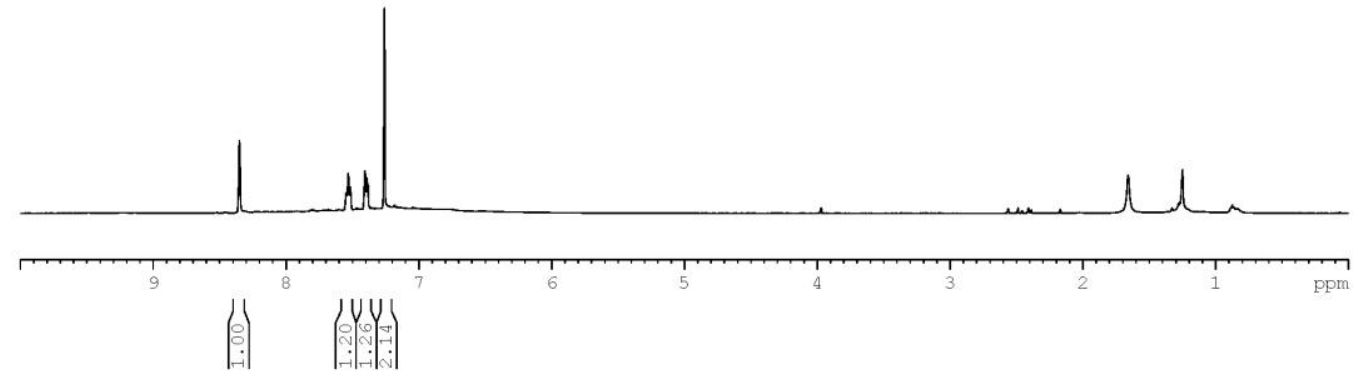

|
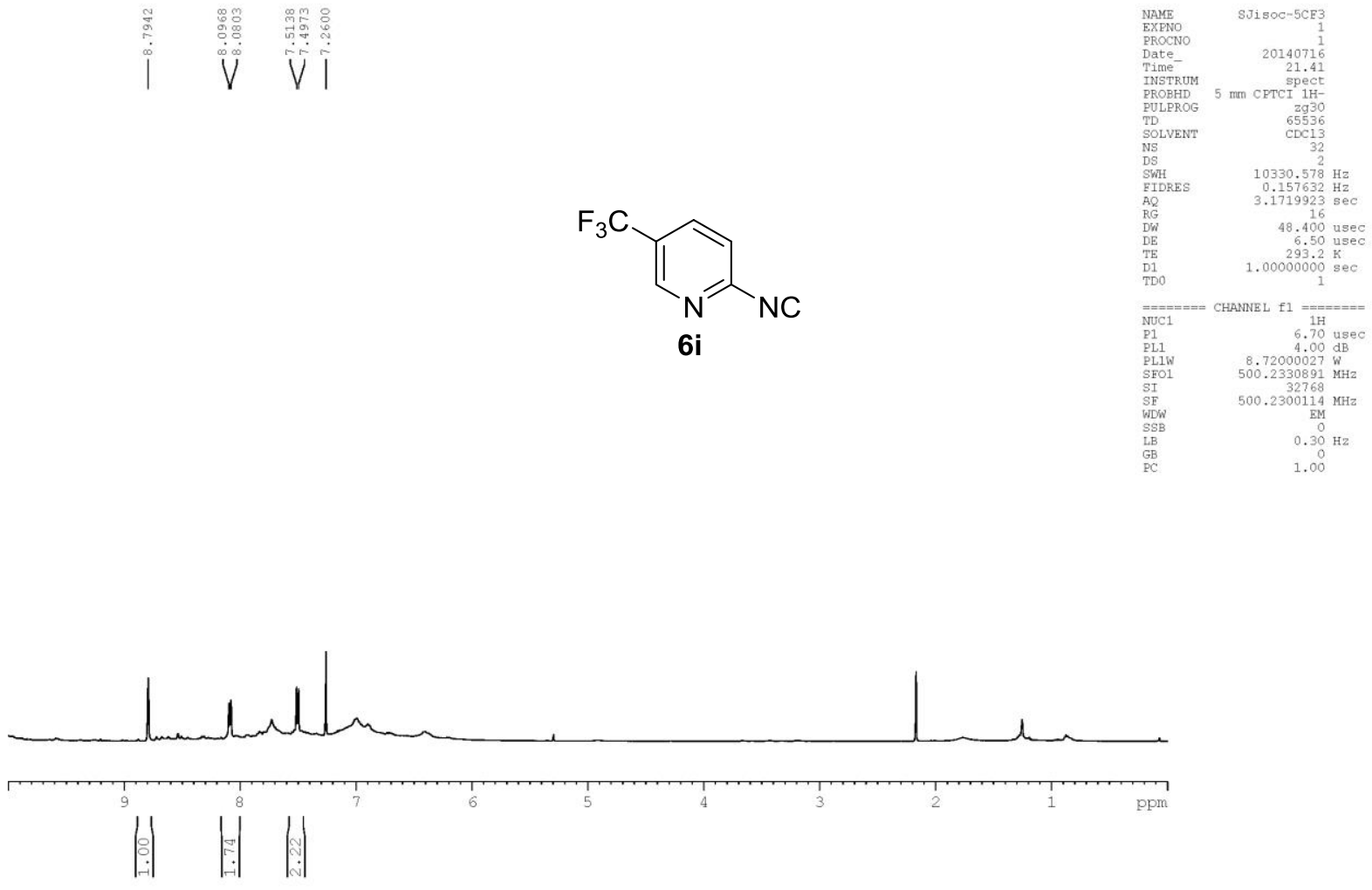

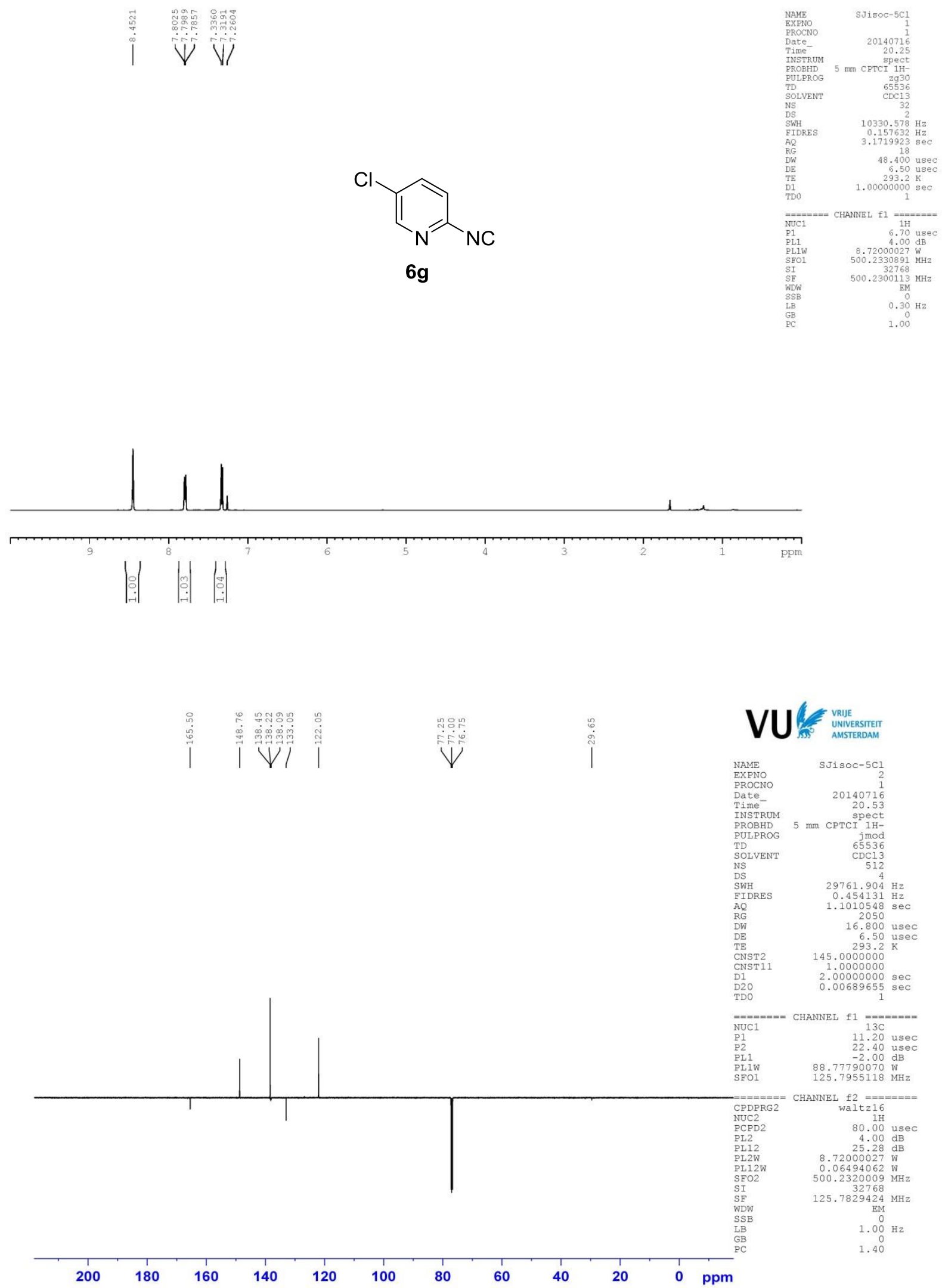

S35 


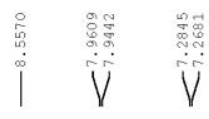
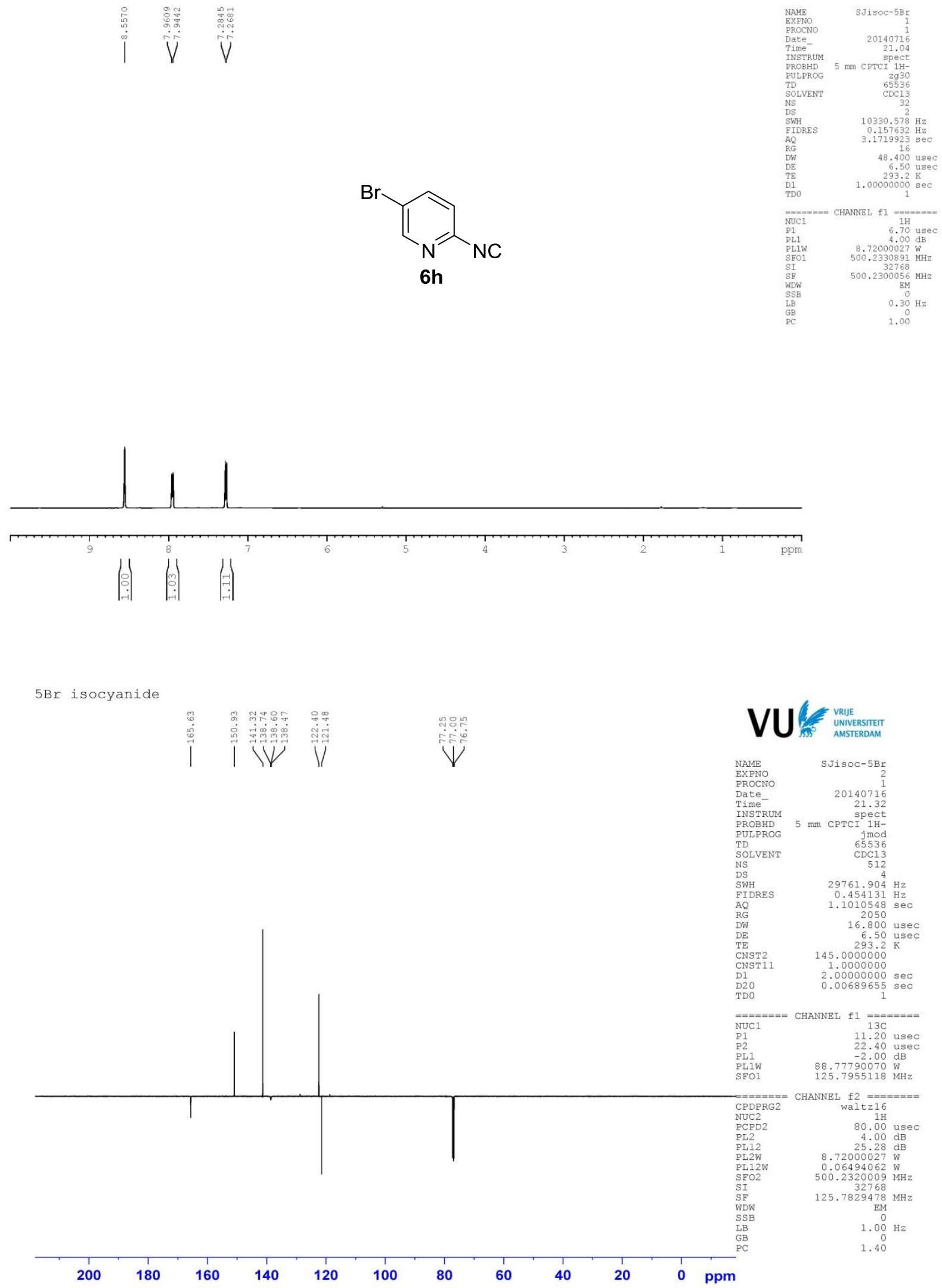

S36 

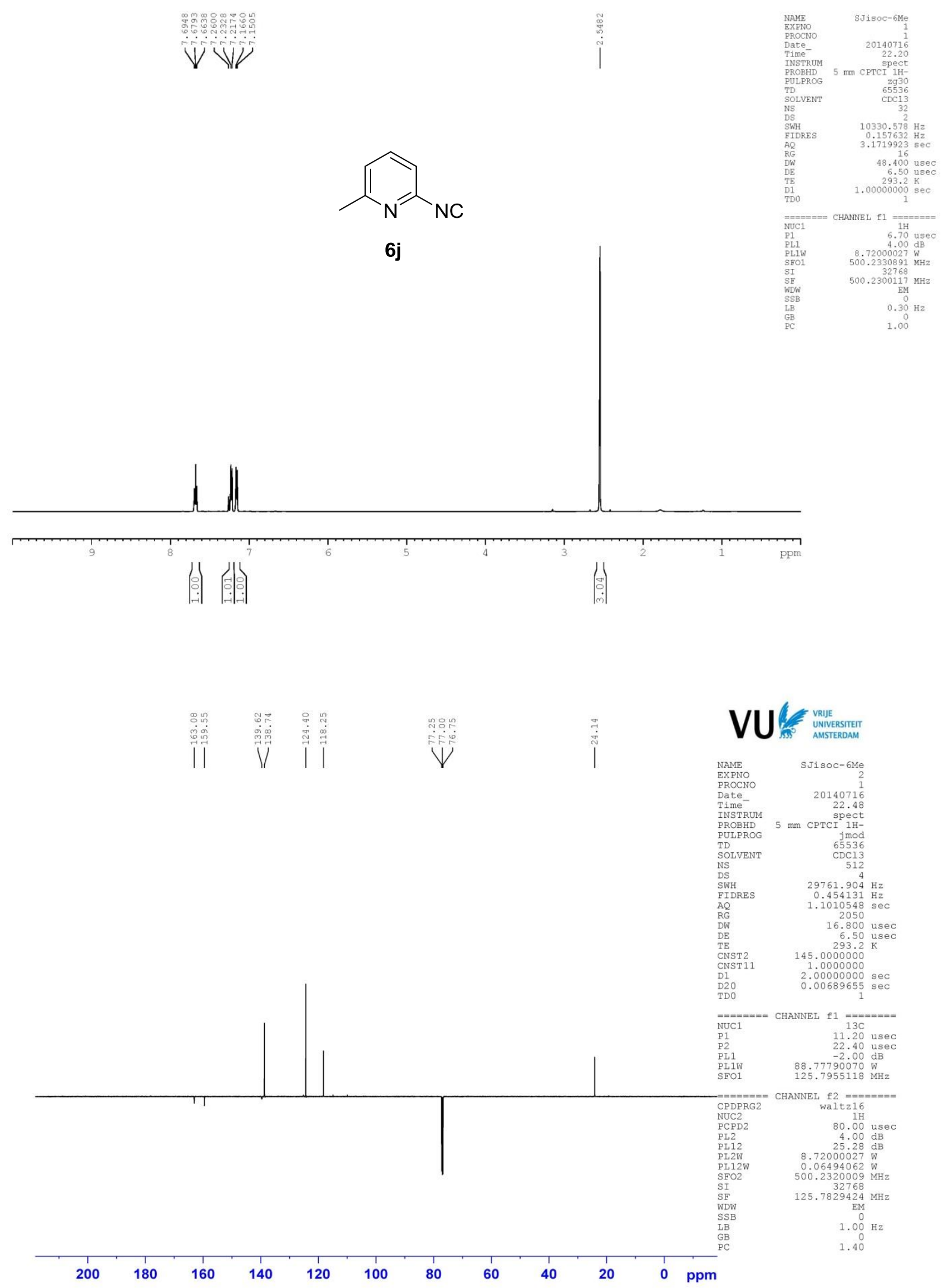


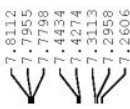

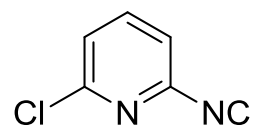

$6 k$
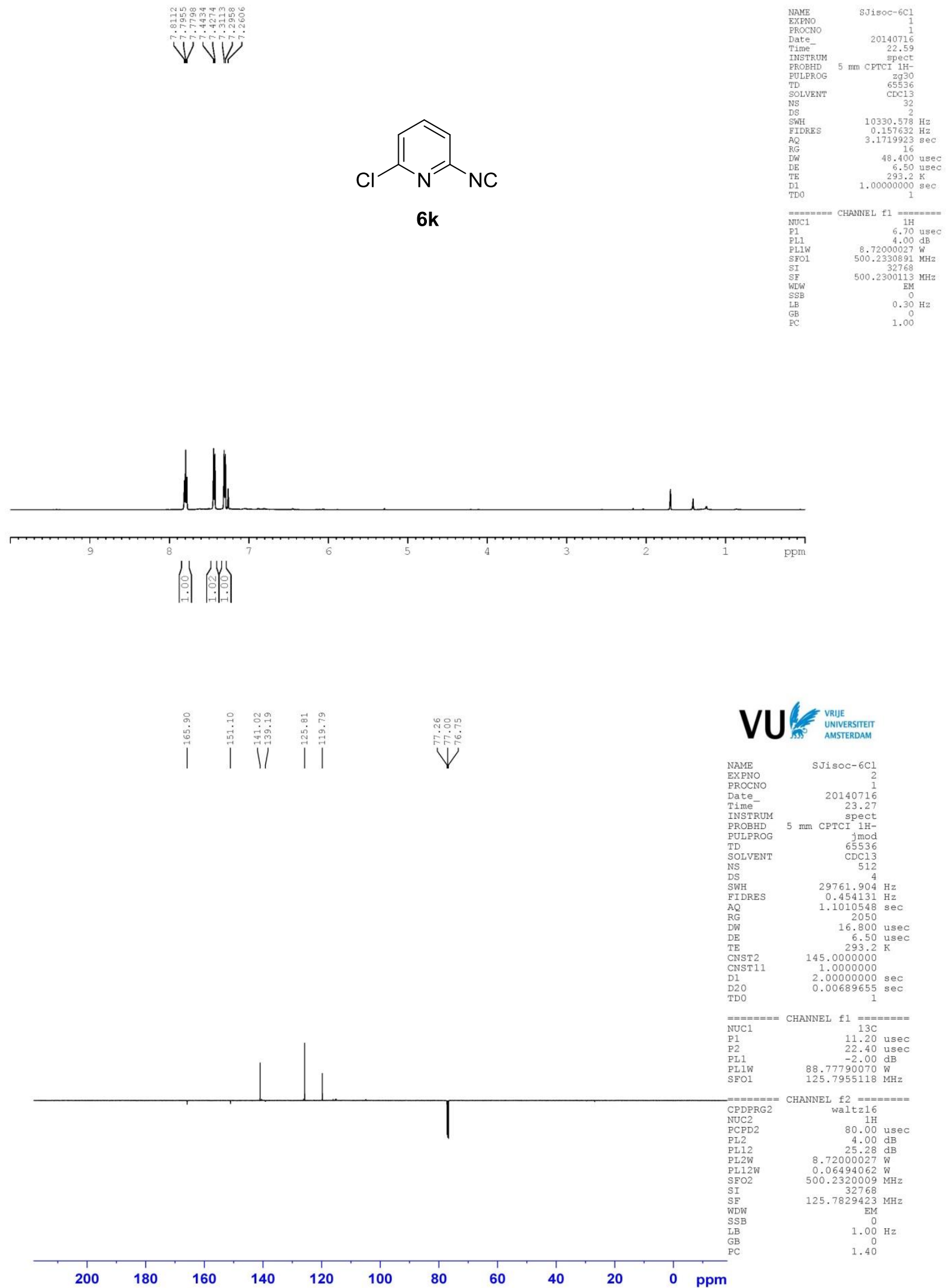

S38 


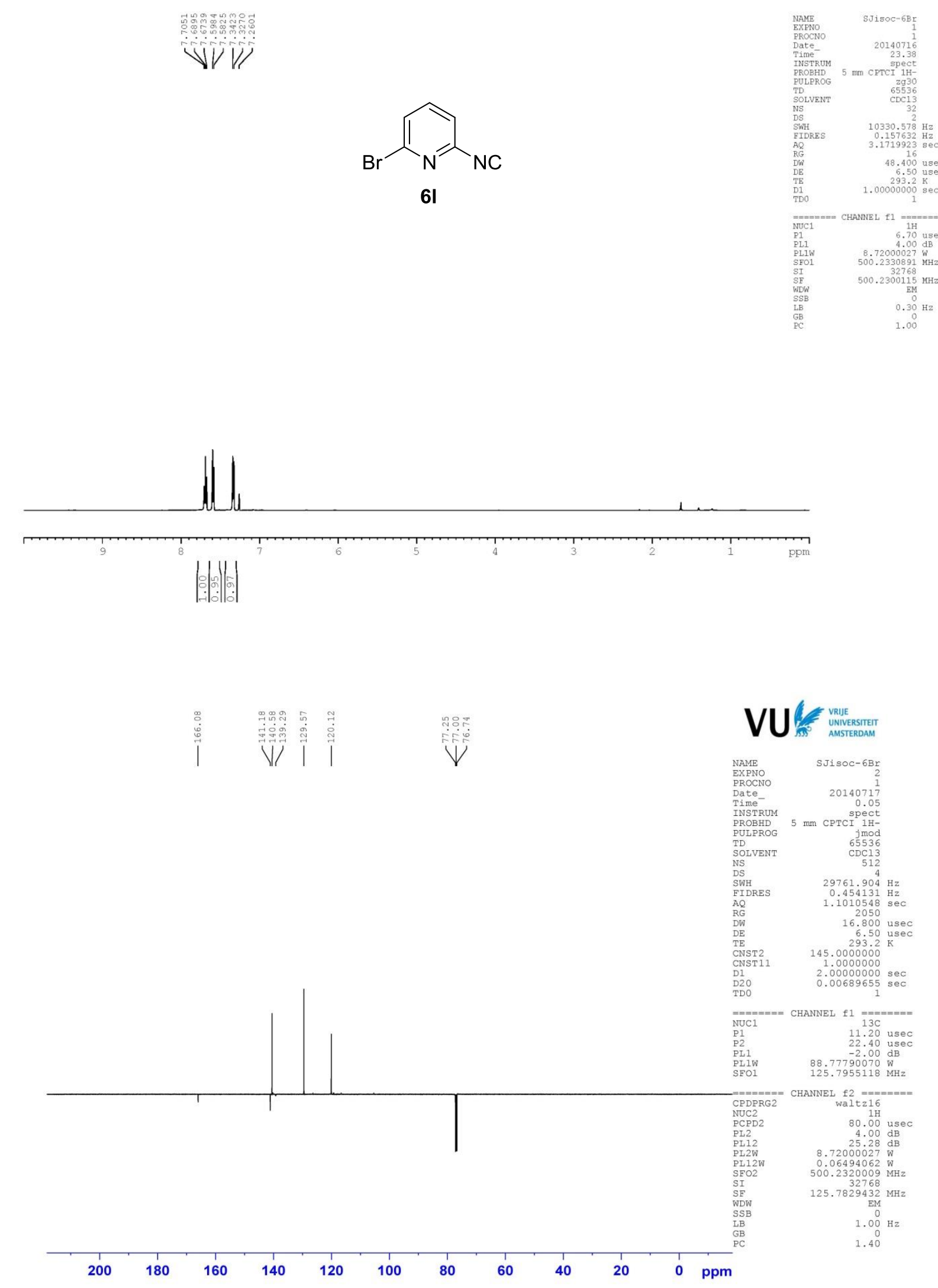

S39 


\section{Ugi products (7)}

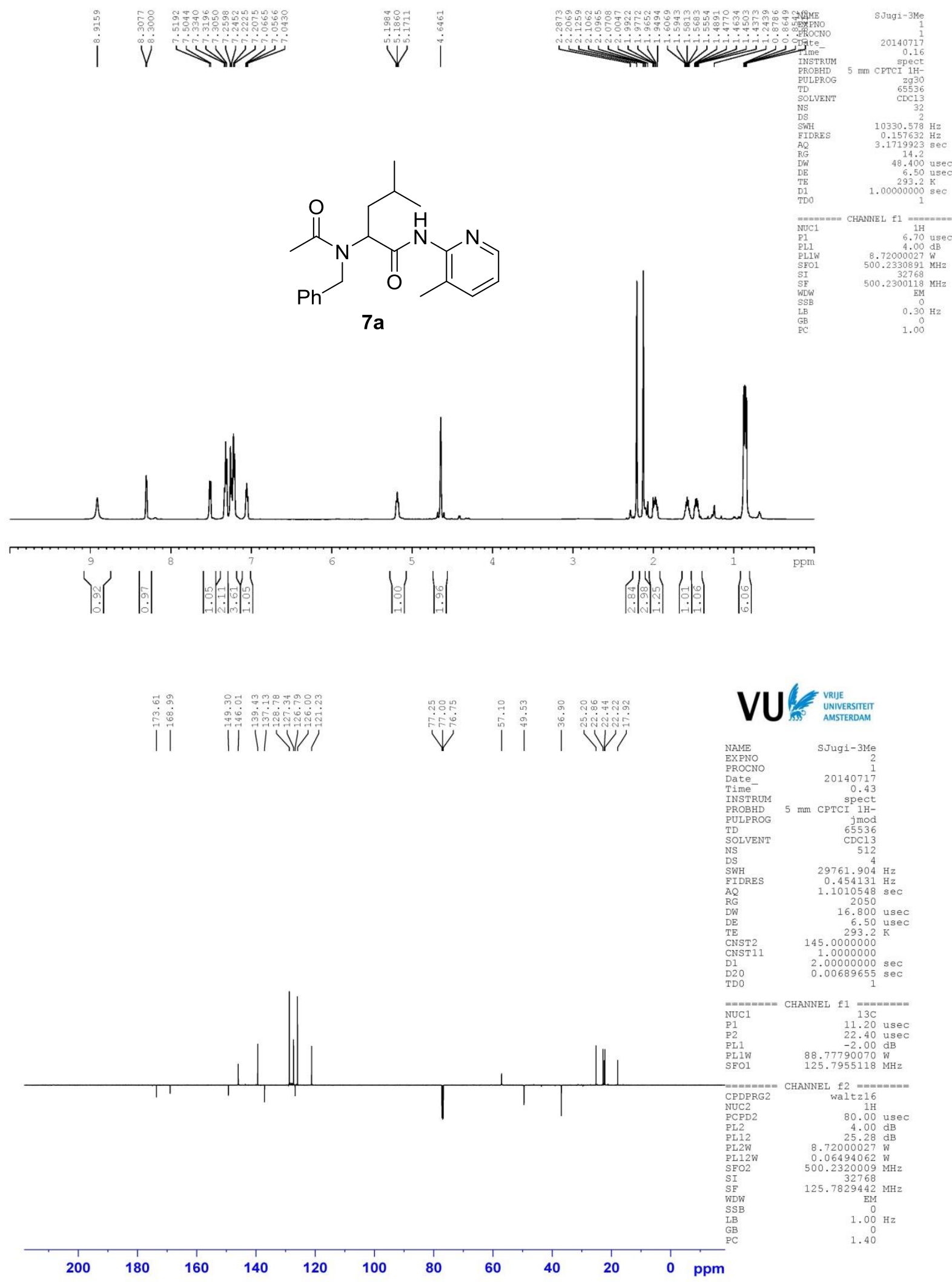




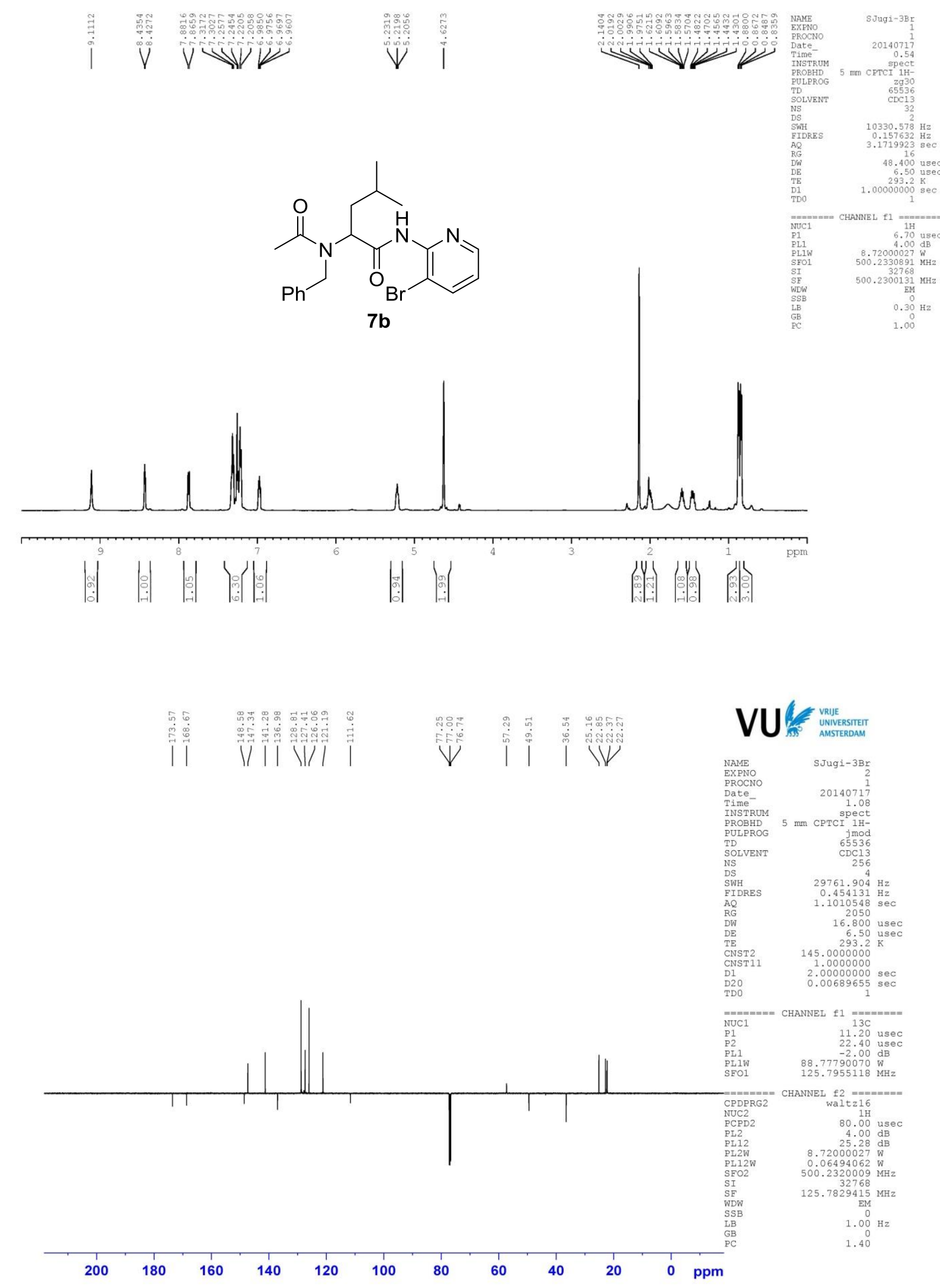

S41 


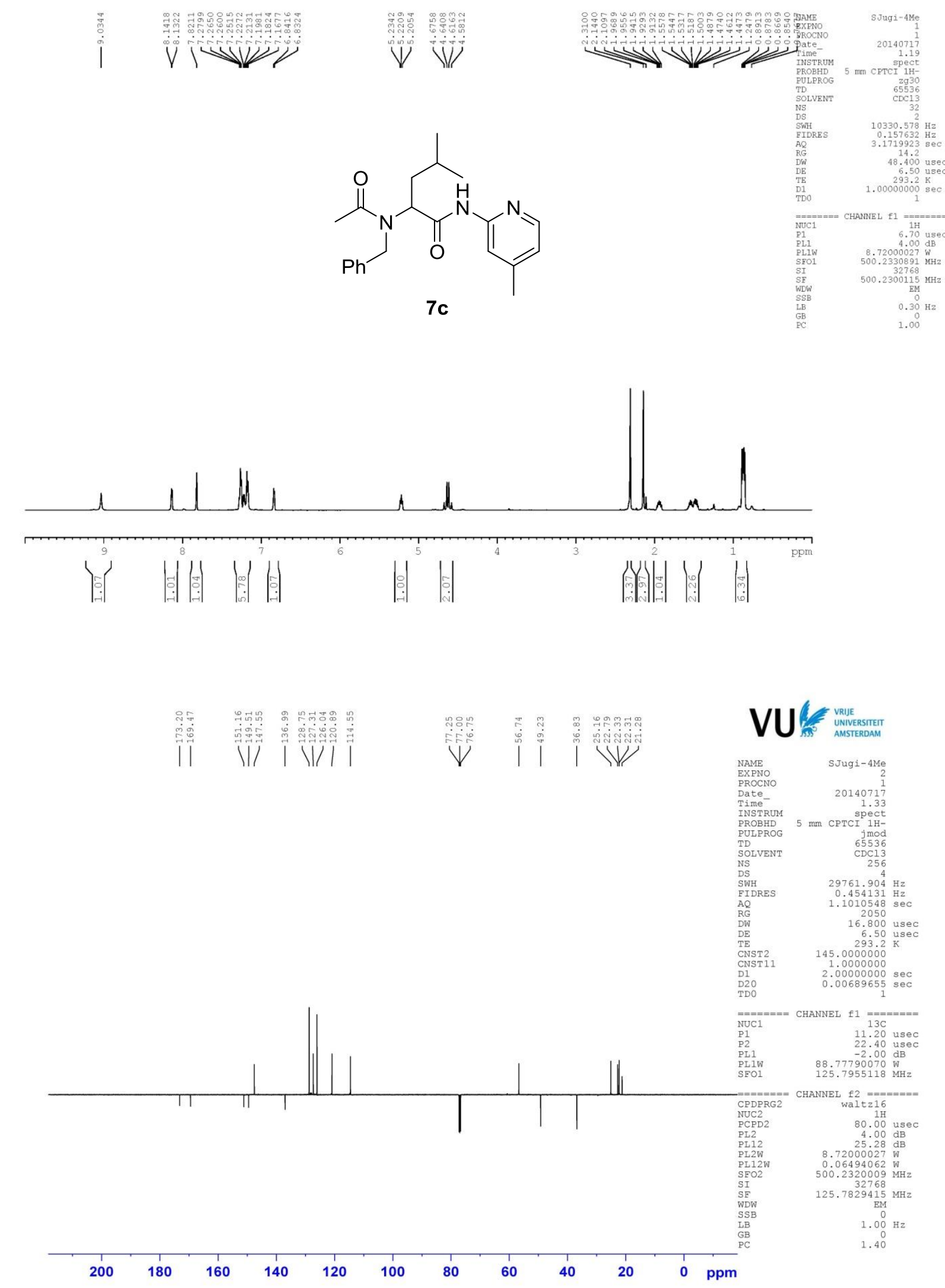

S42 

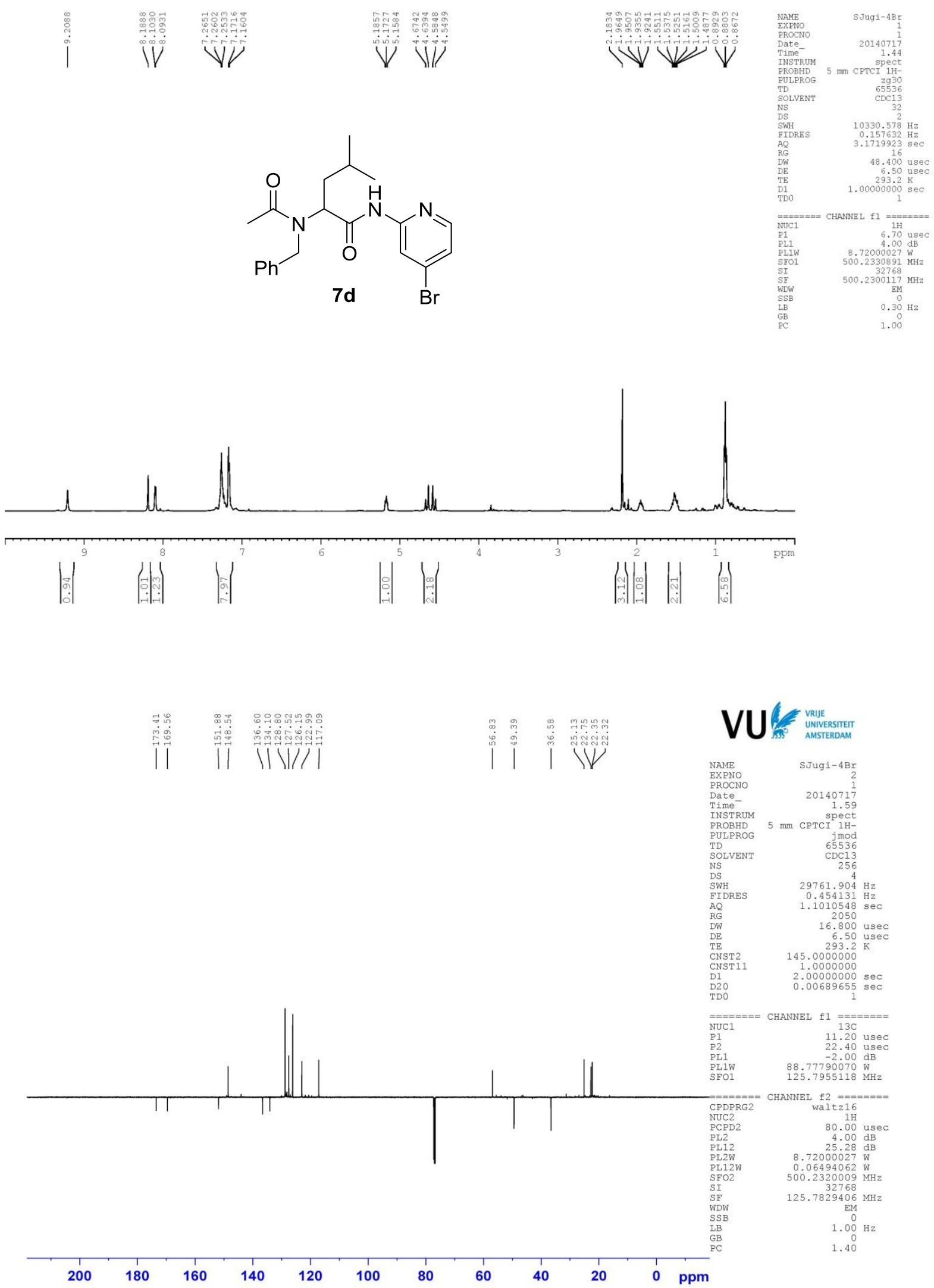

S43 

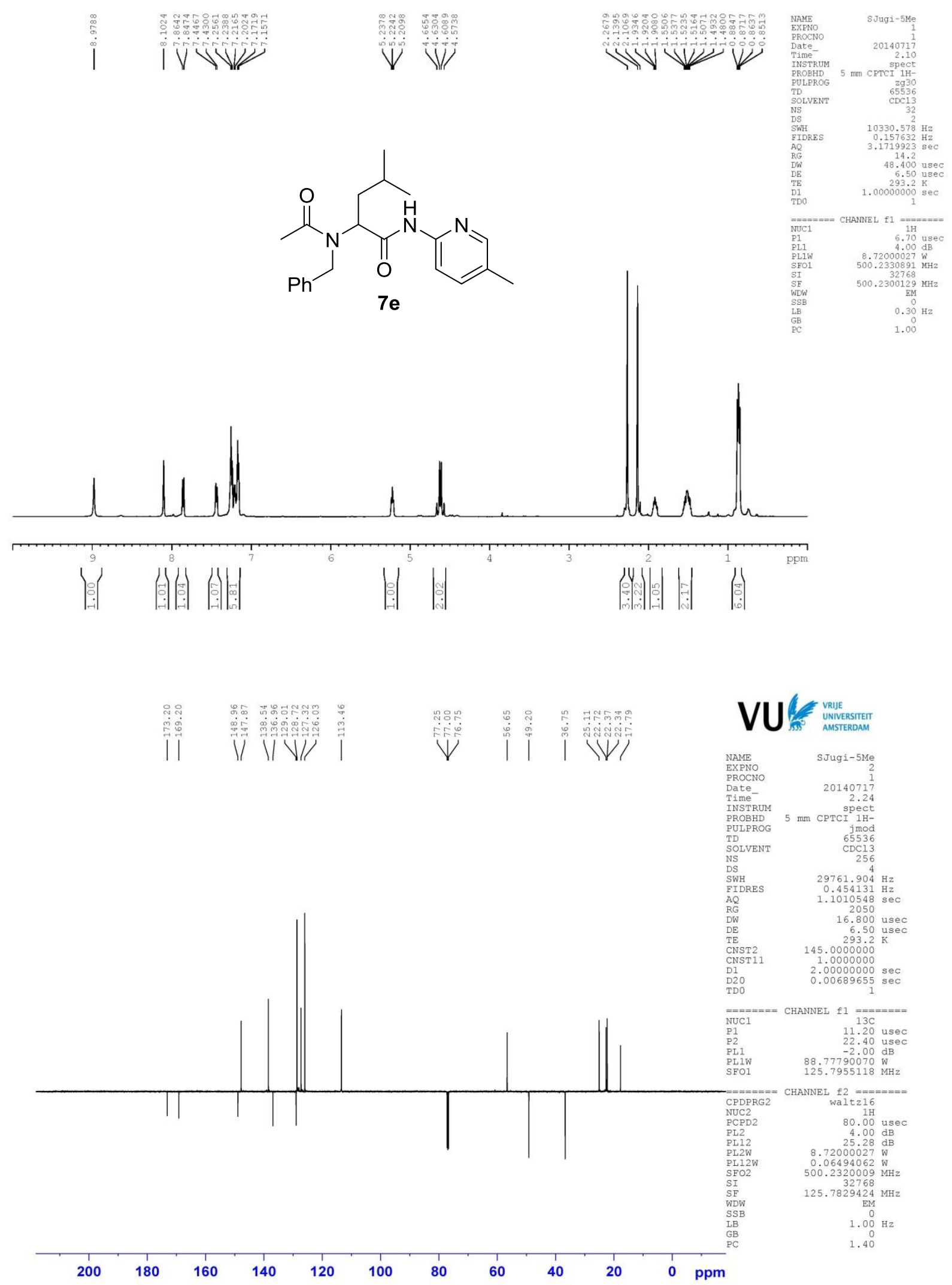


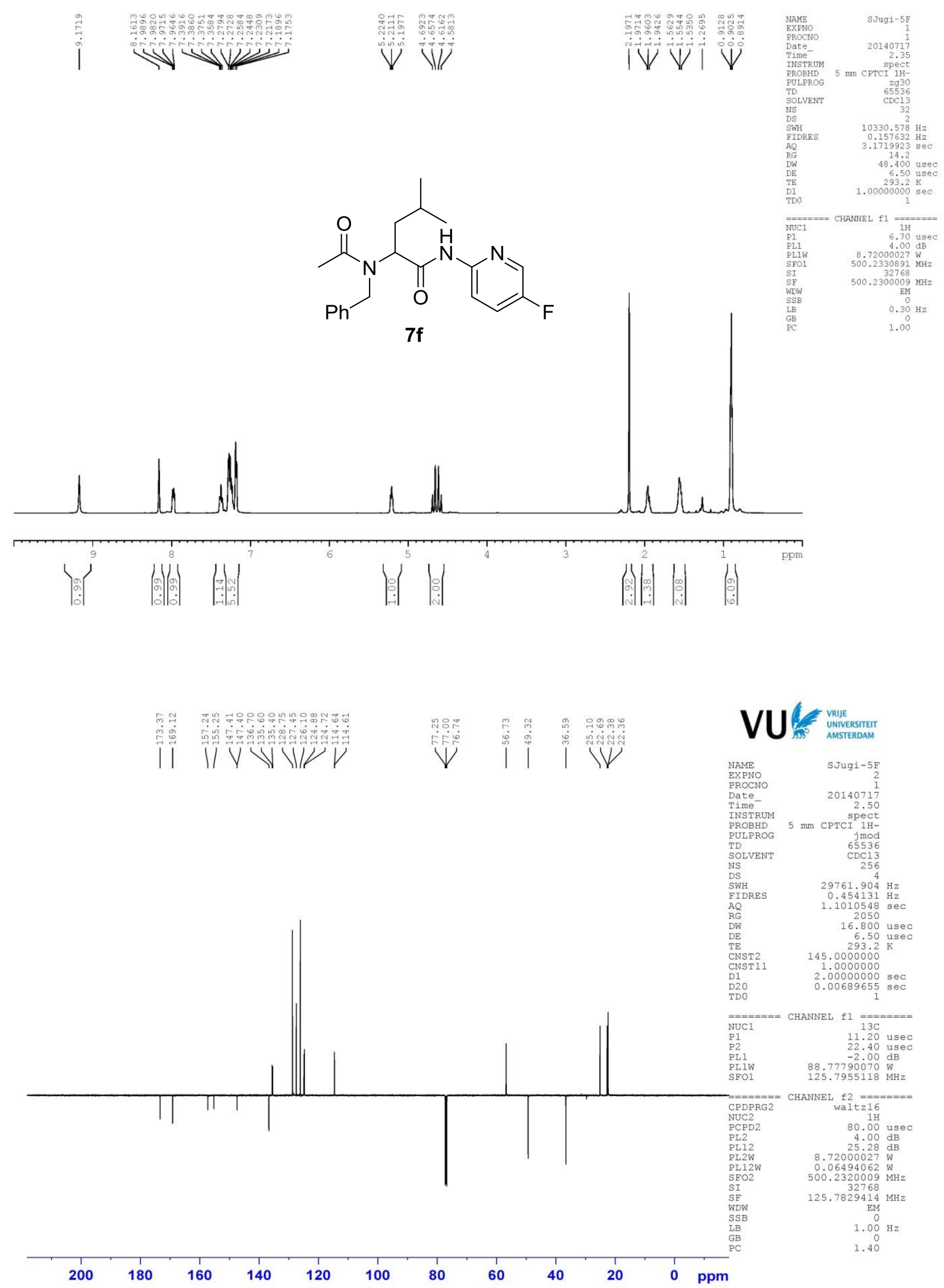




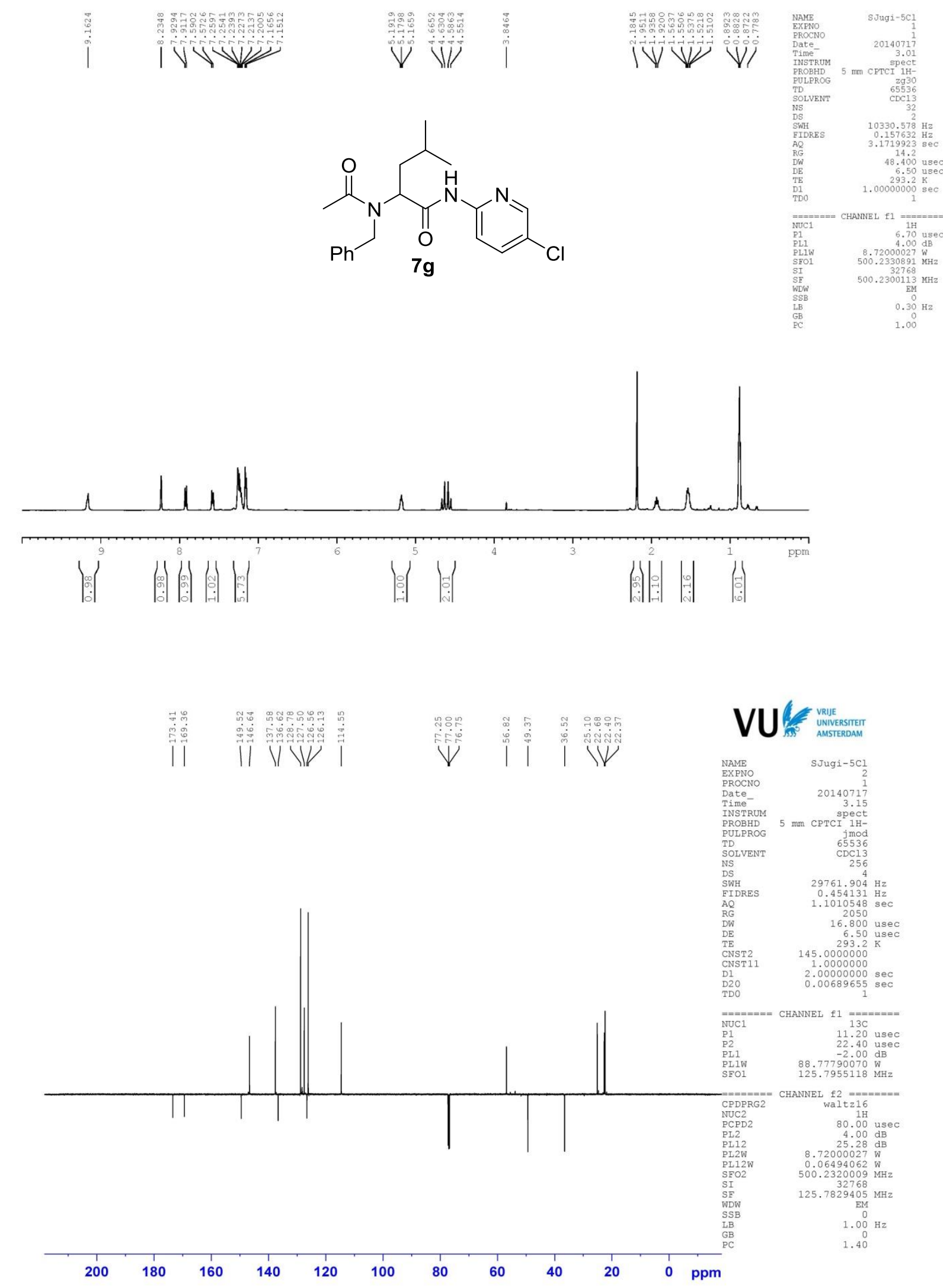




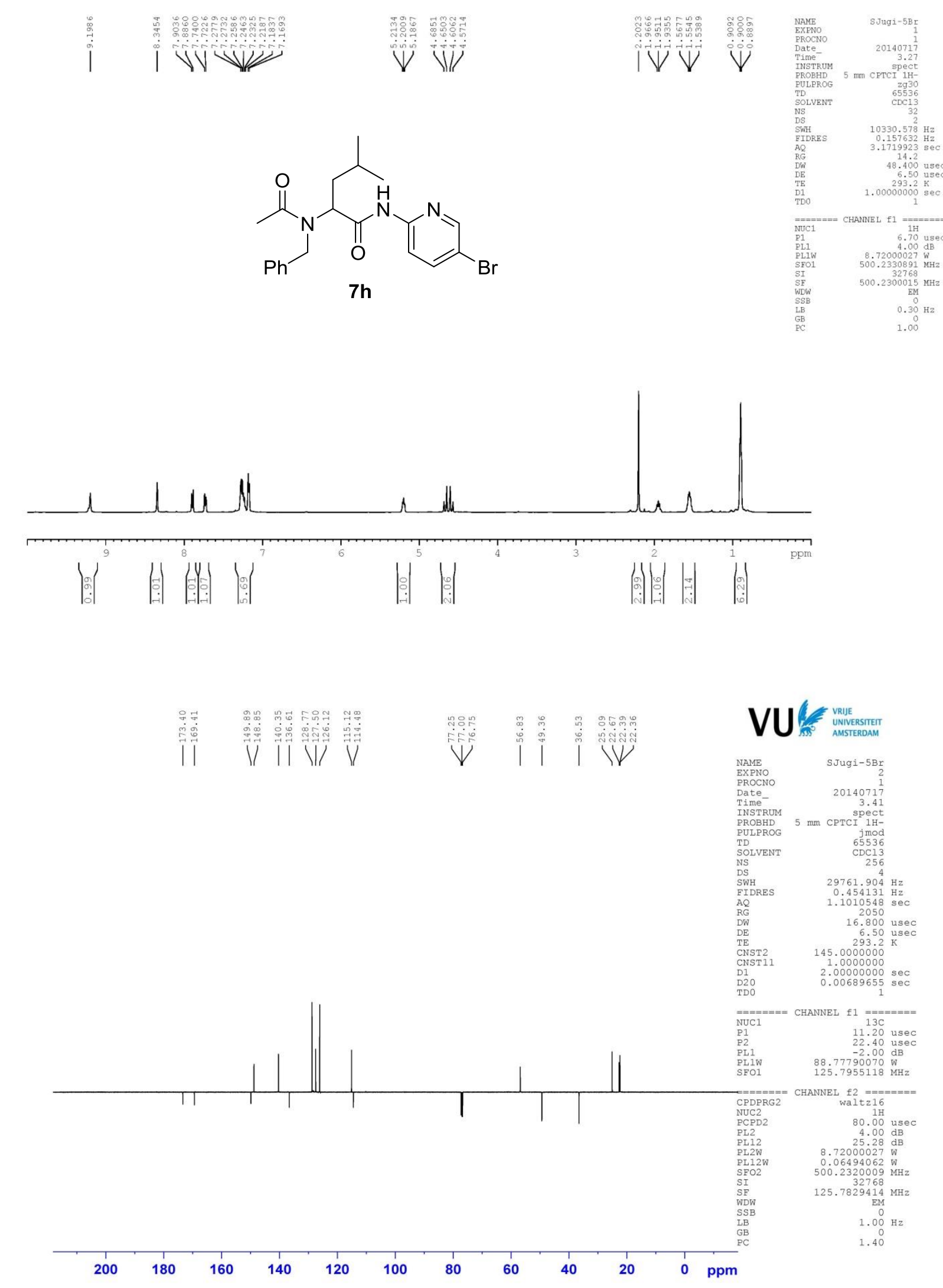




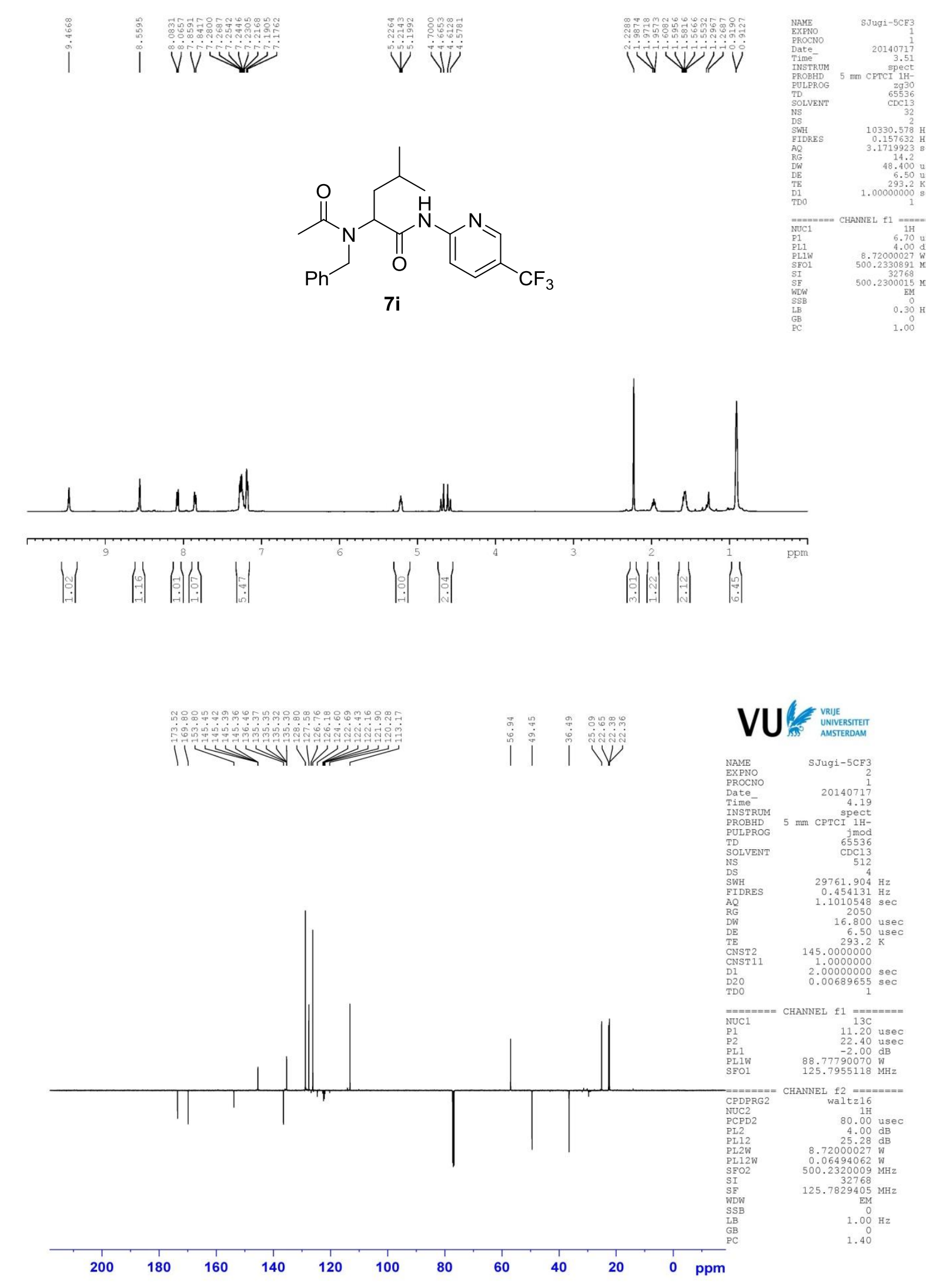




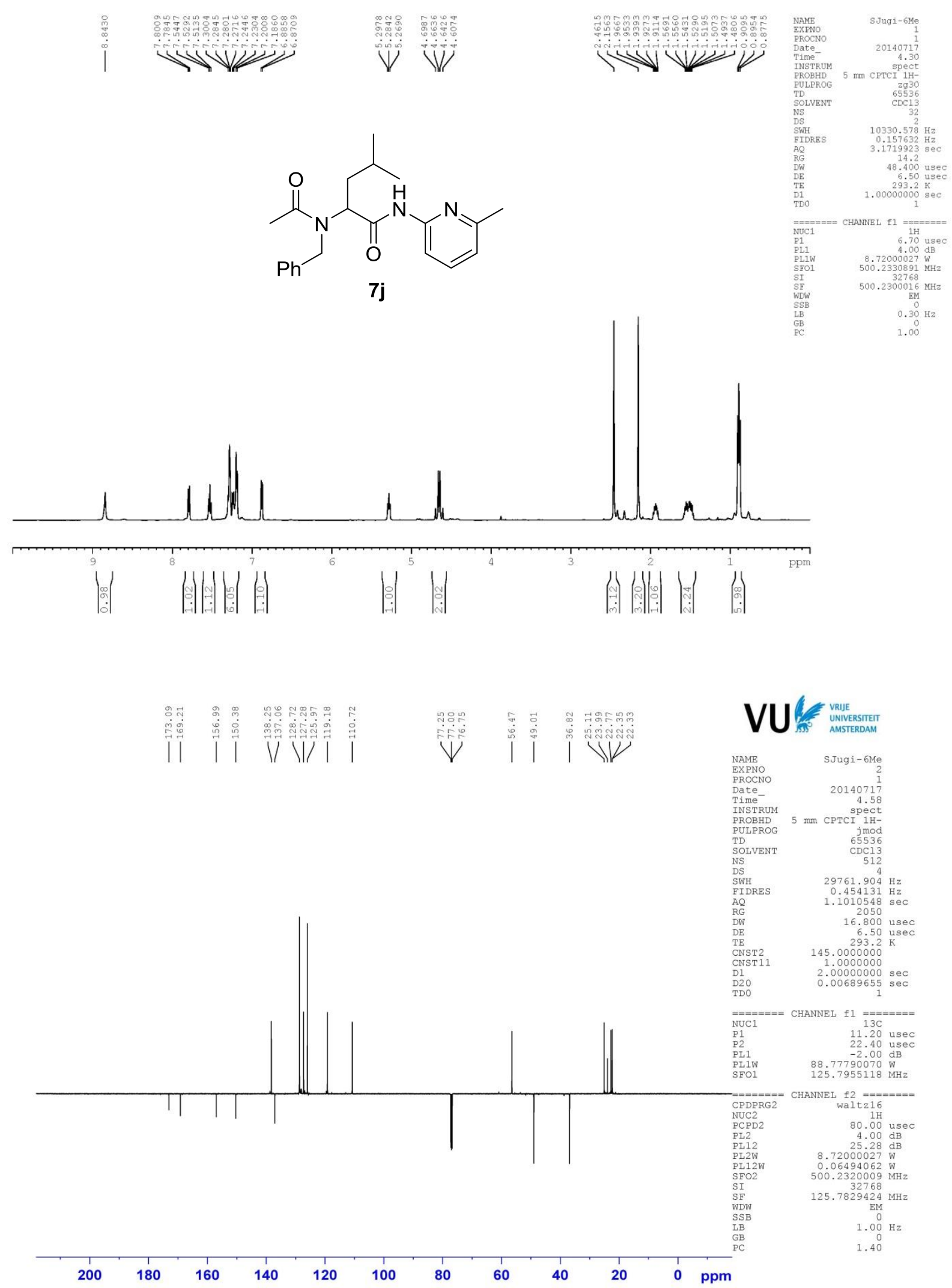




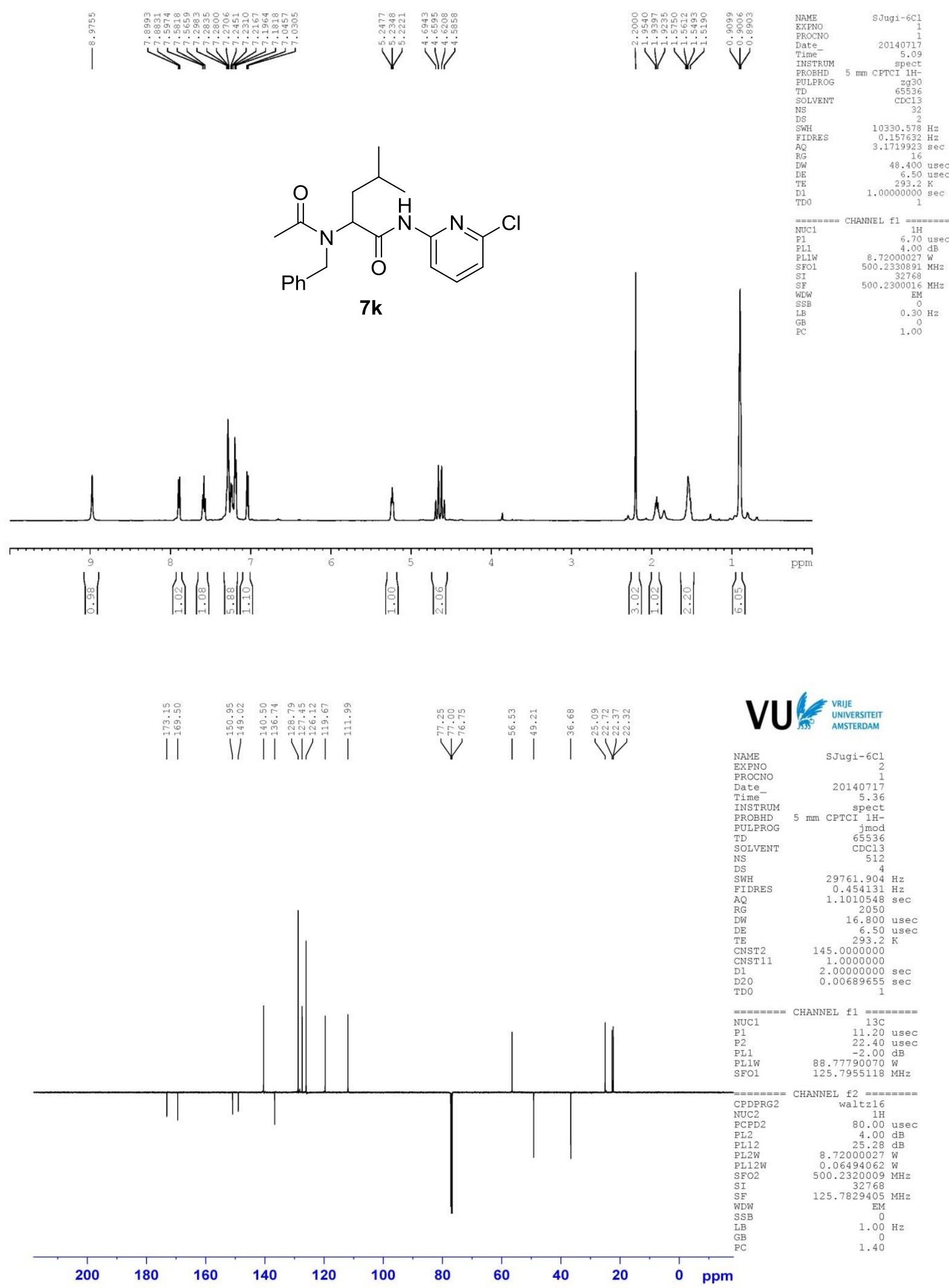



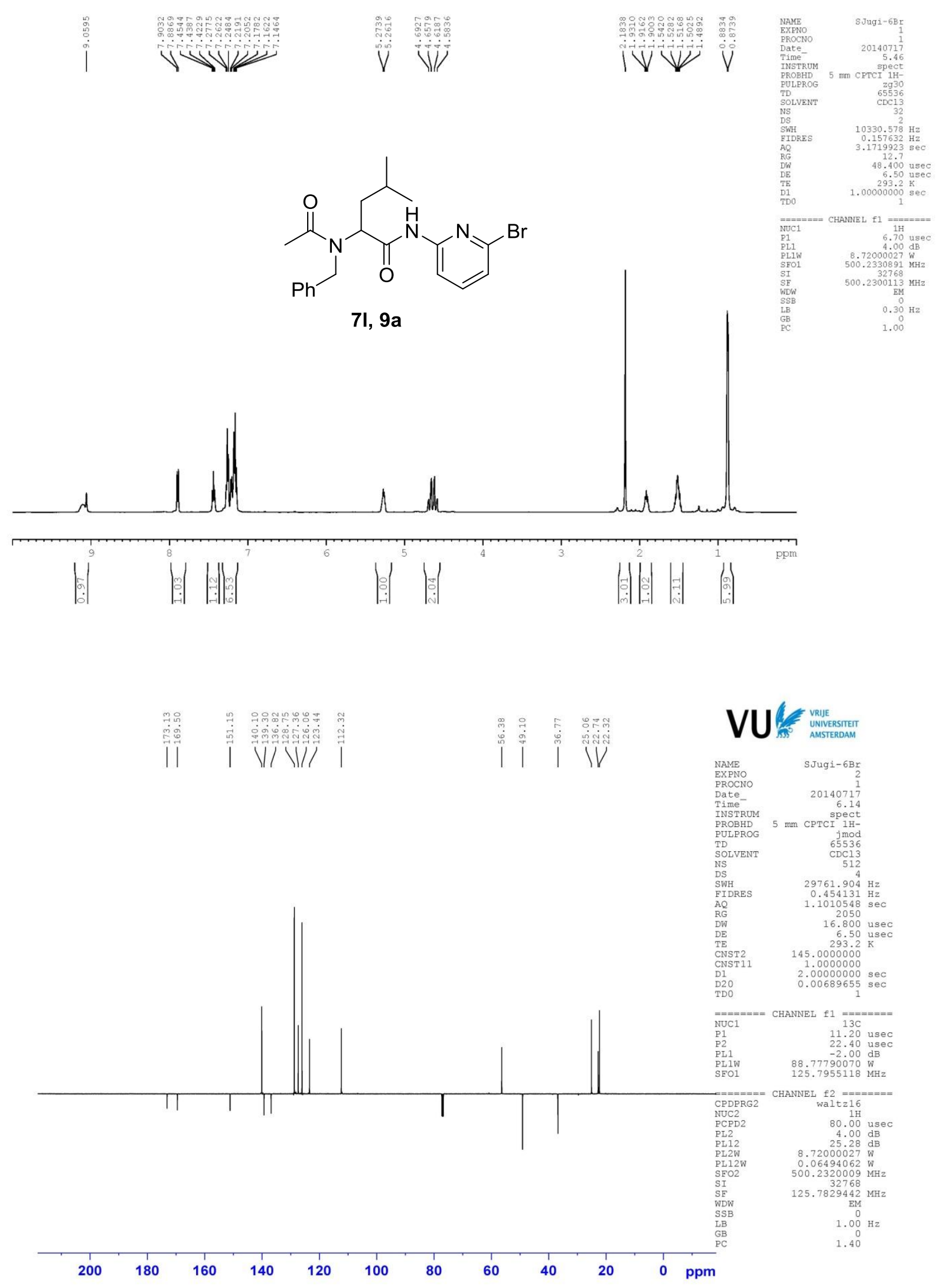

S51 


\section{Ugi products (9)}

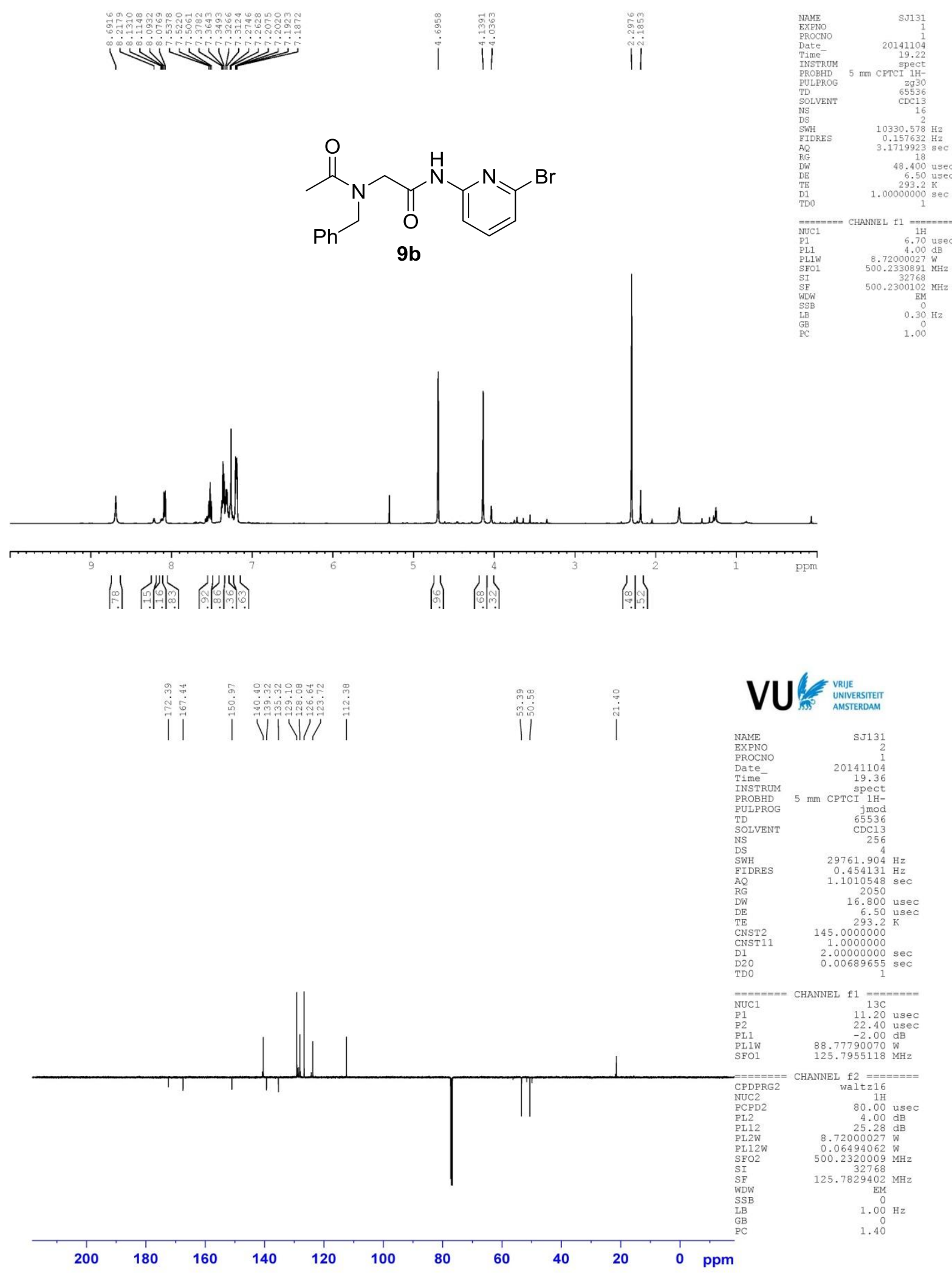




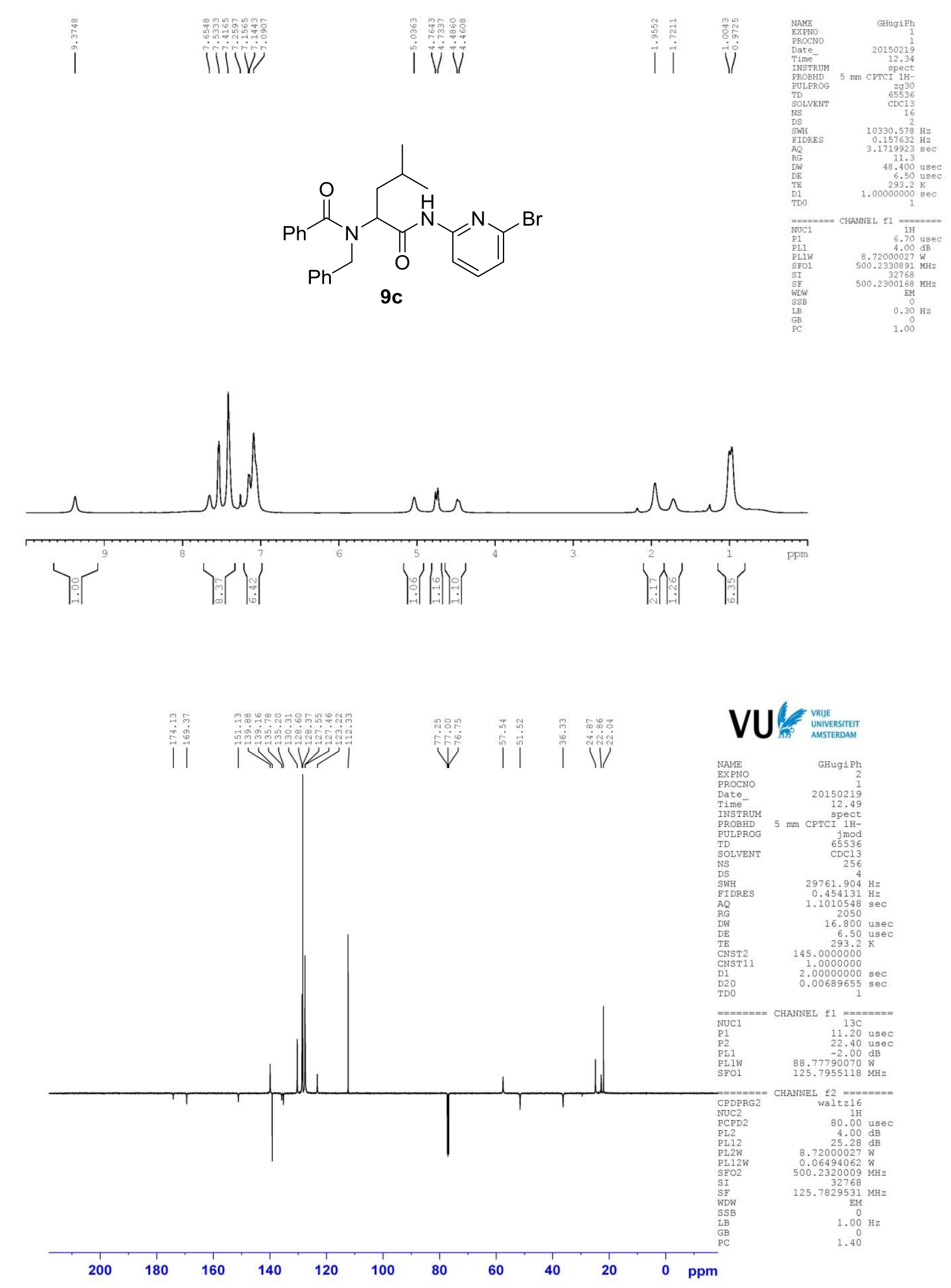

S53 

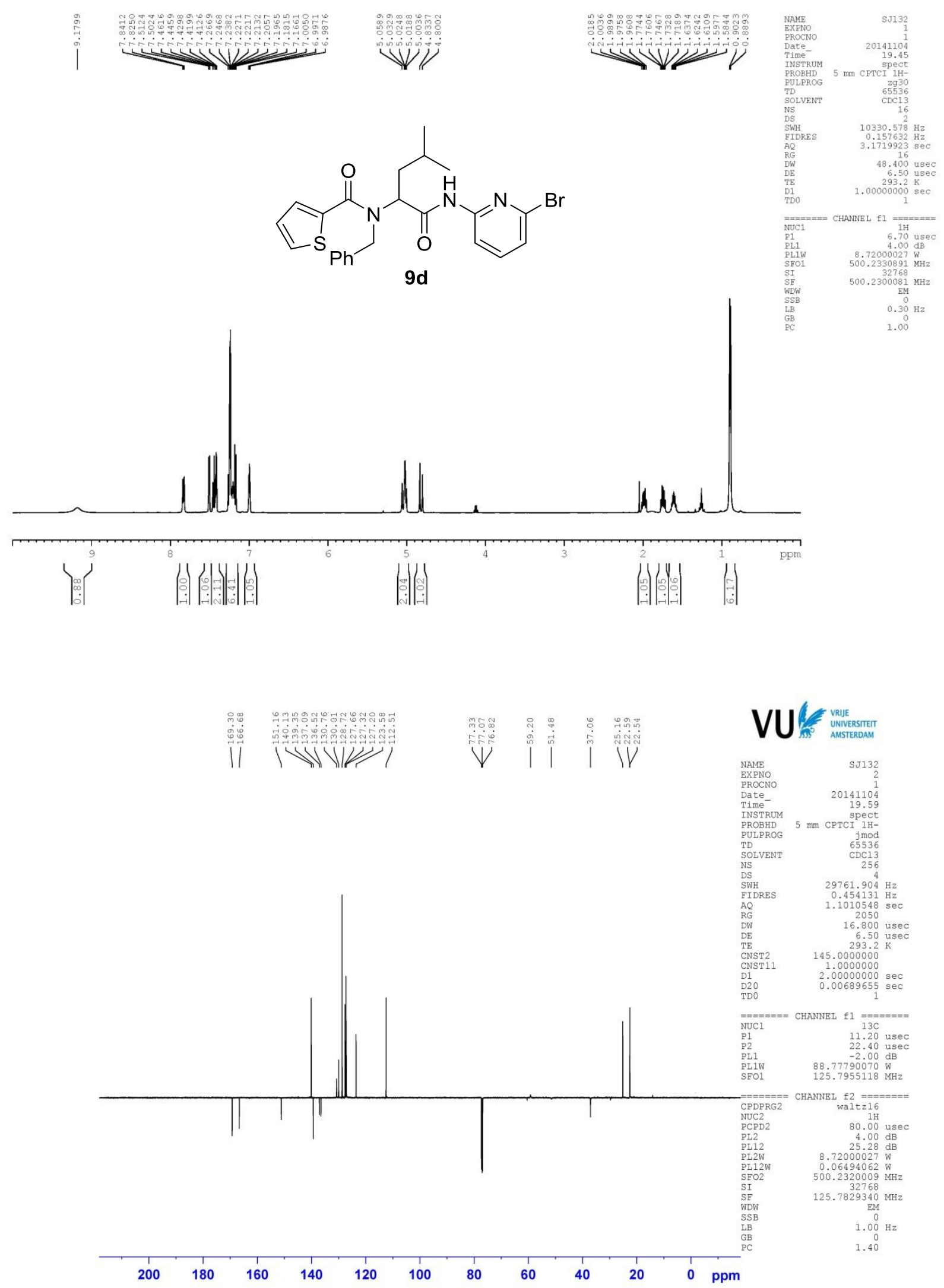

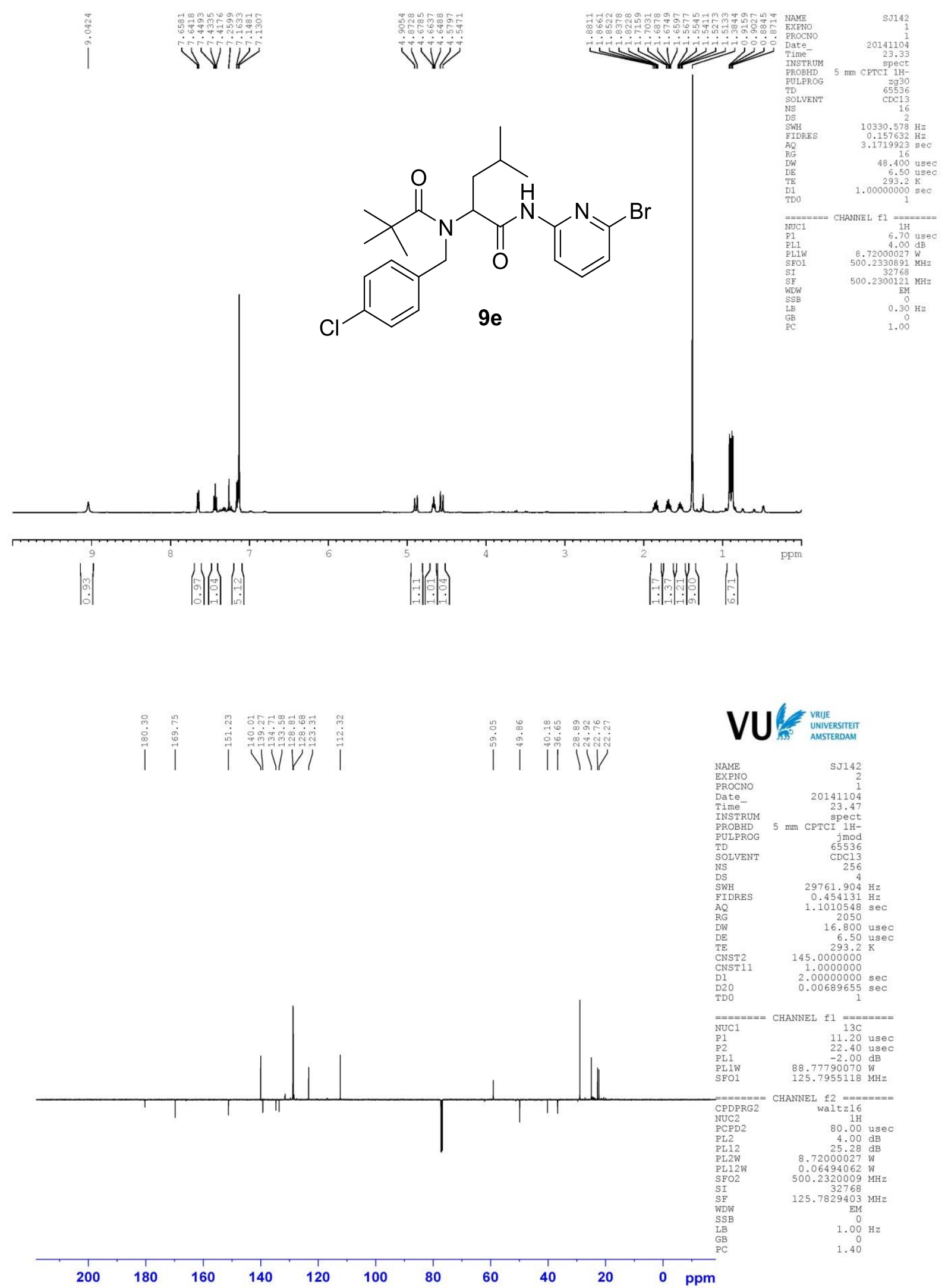

S55 


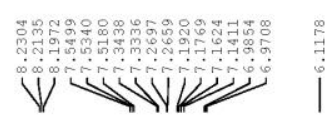

ที่

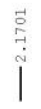<smiles>CC(=O)N(Cc1ccccc1)C(C(=O)Nc1cccc(Br)n1)c1ccccc1</smiles>
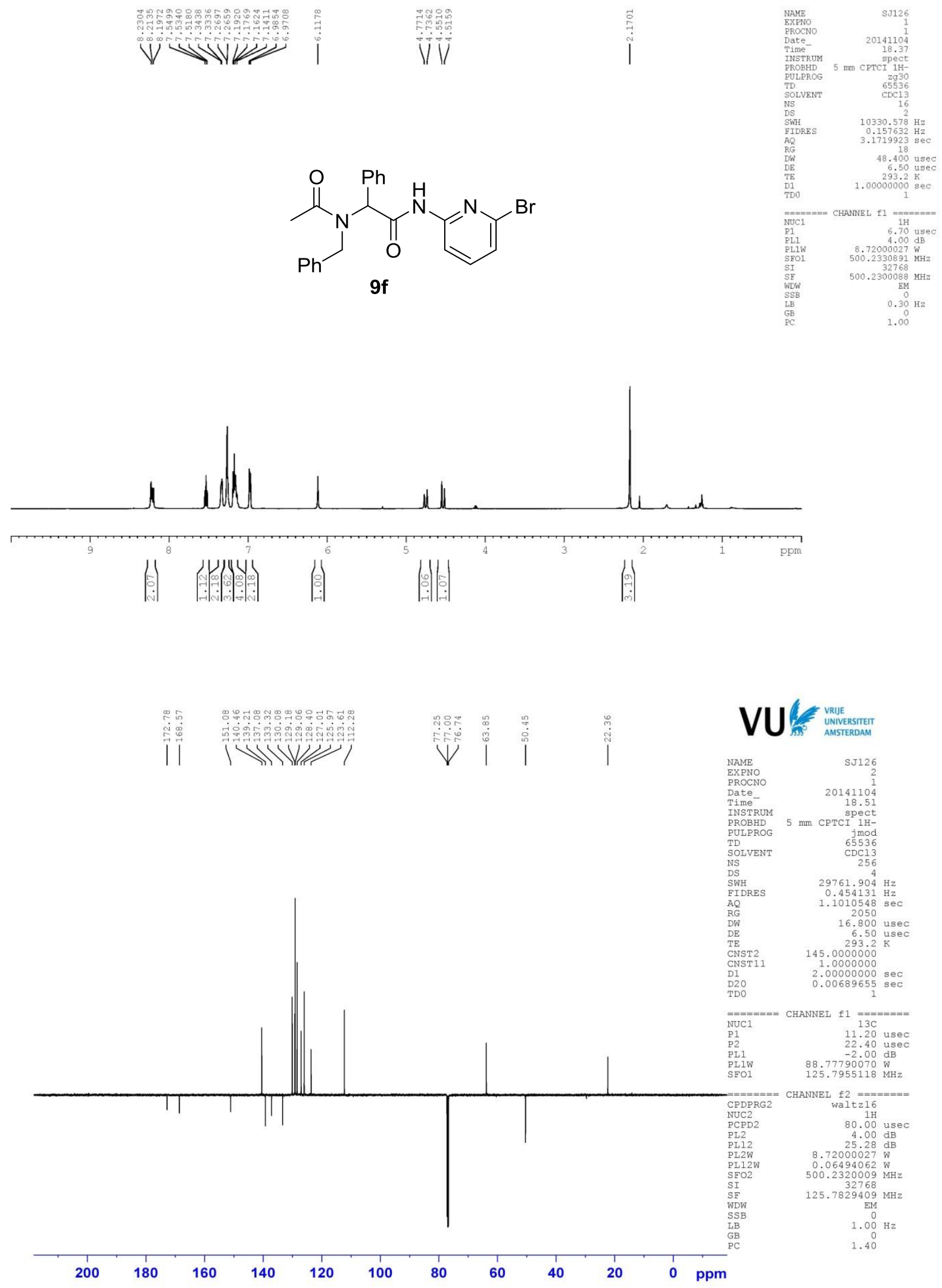

S56 

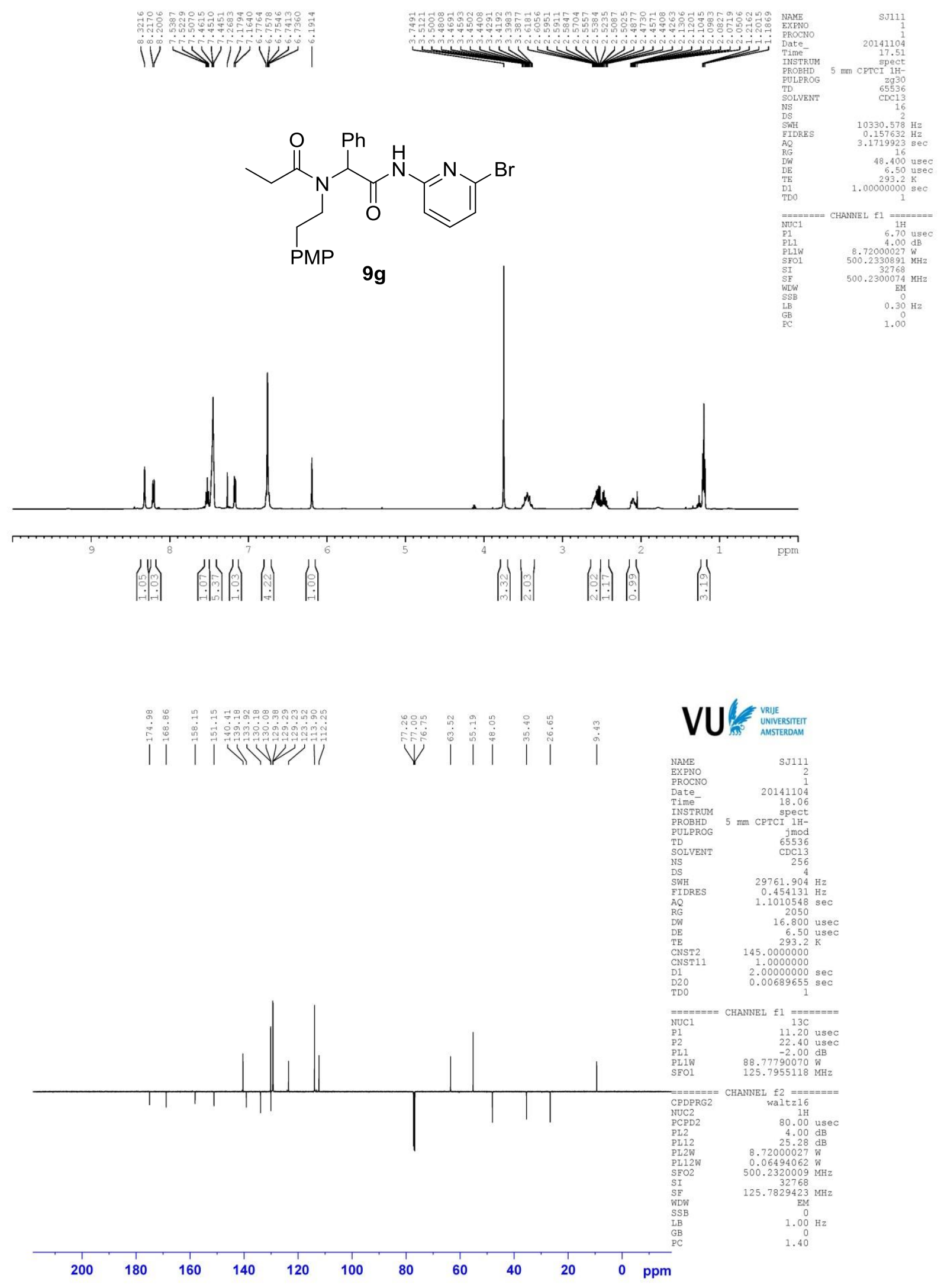


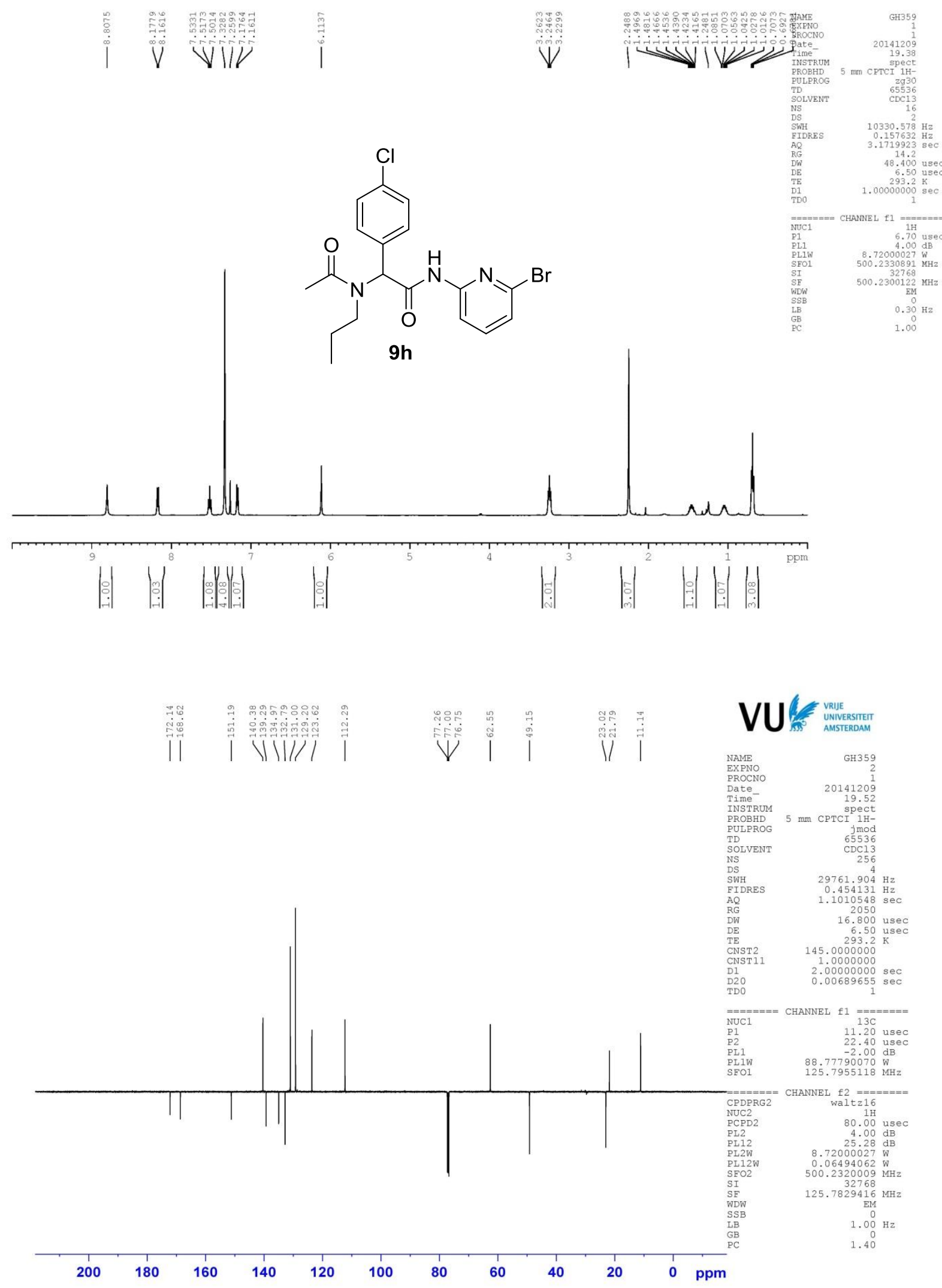

S58 


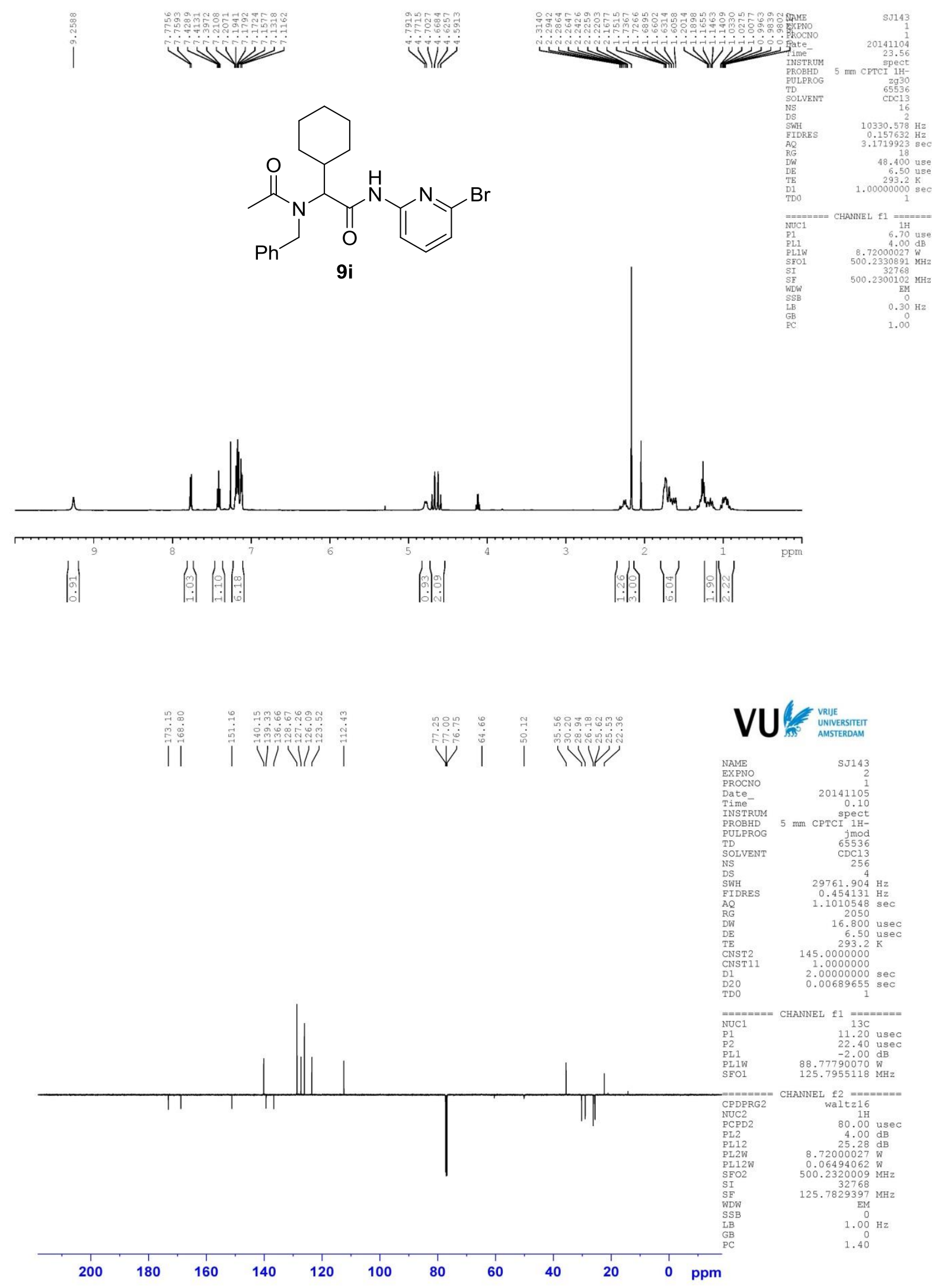

S59 


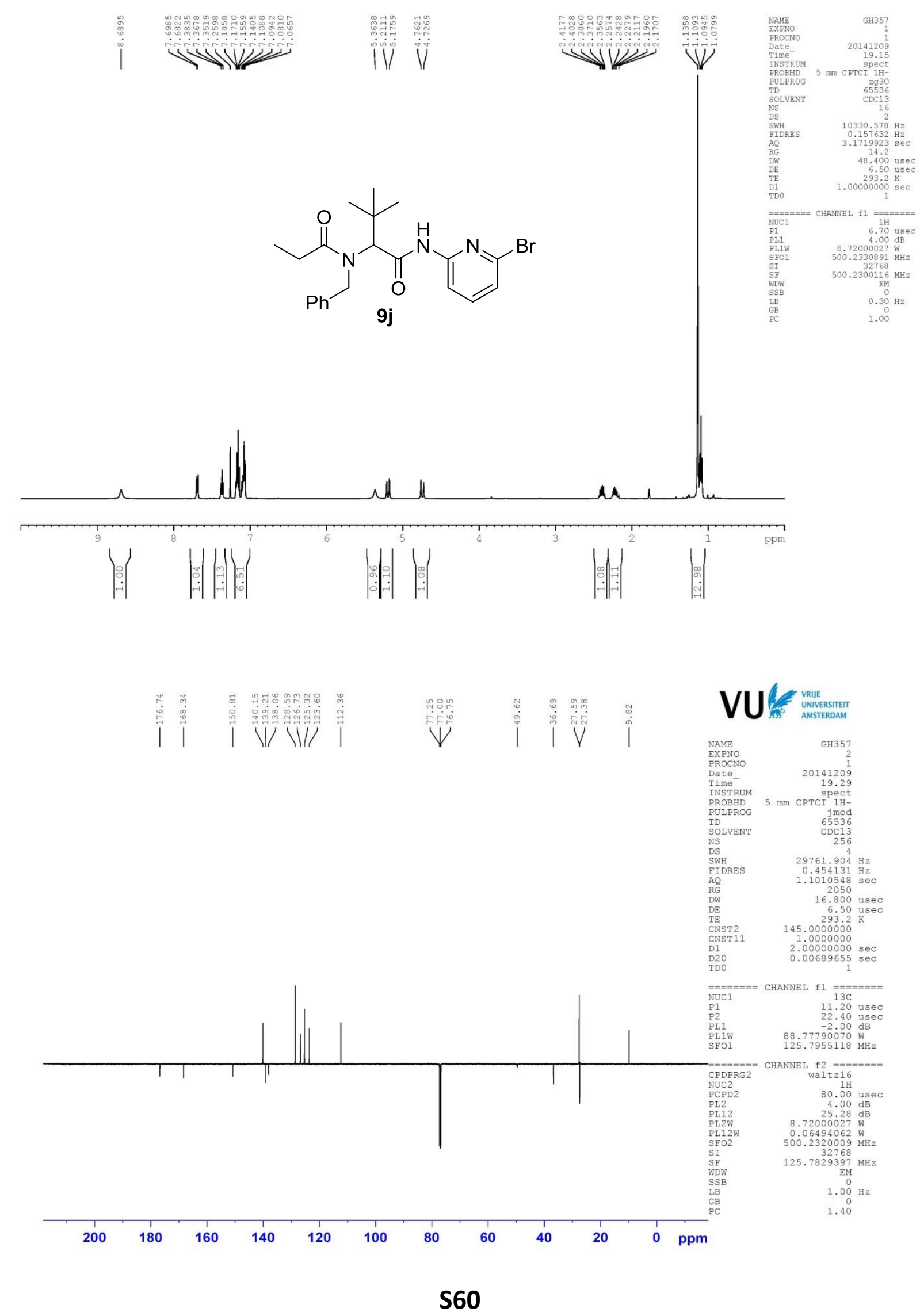




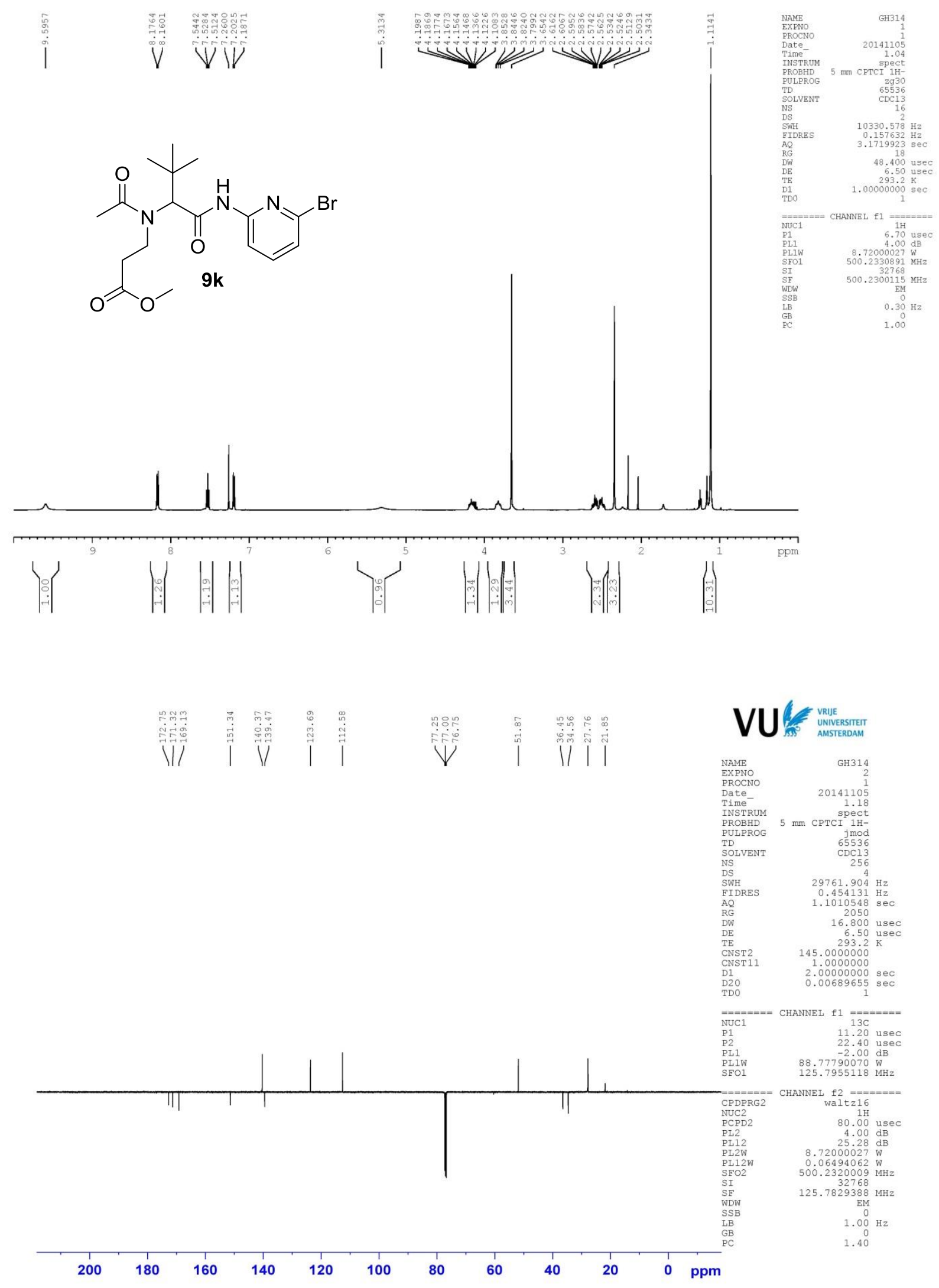

S61 


\section{Hydrolyzed Ugi products (8)}

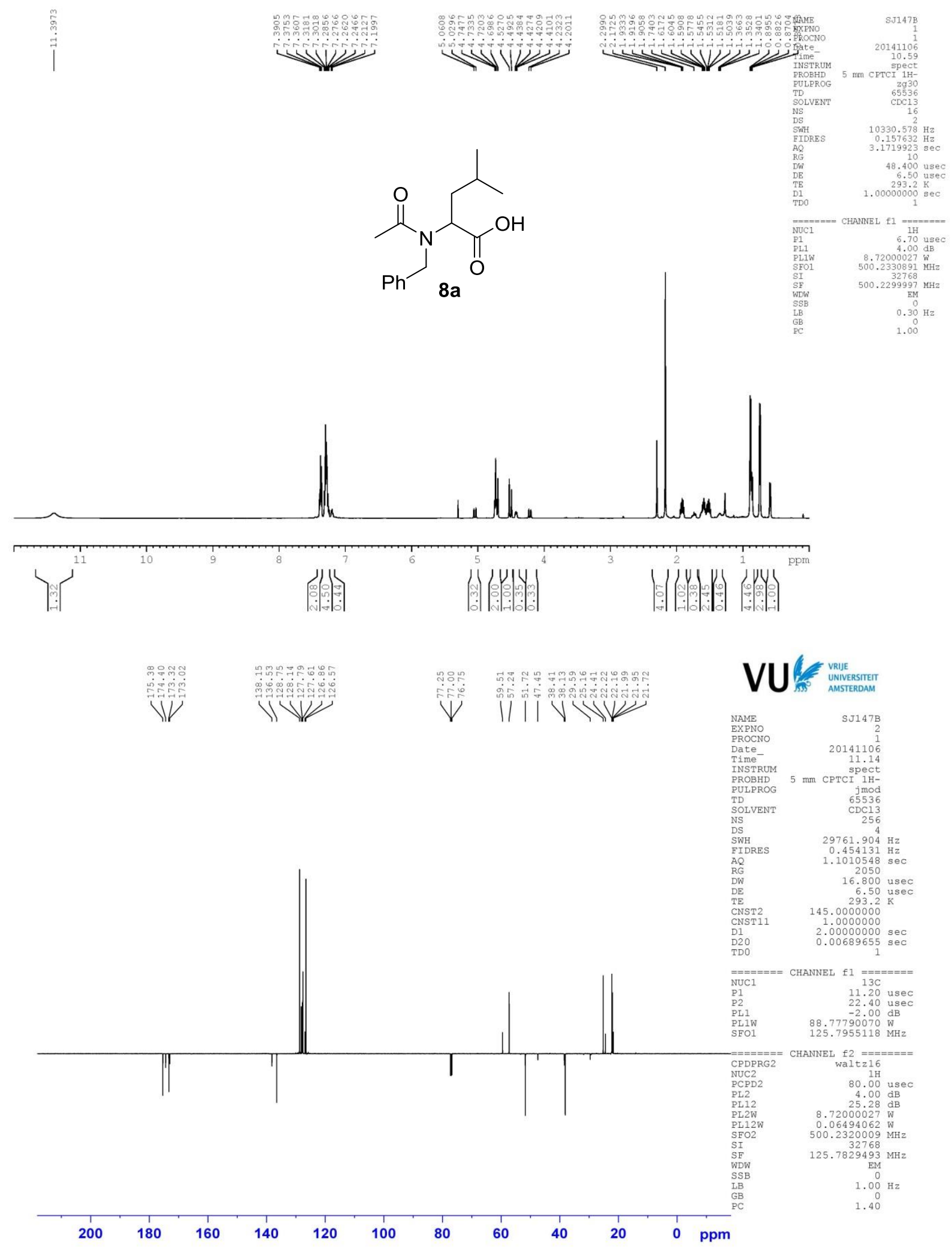




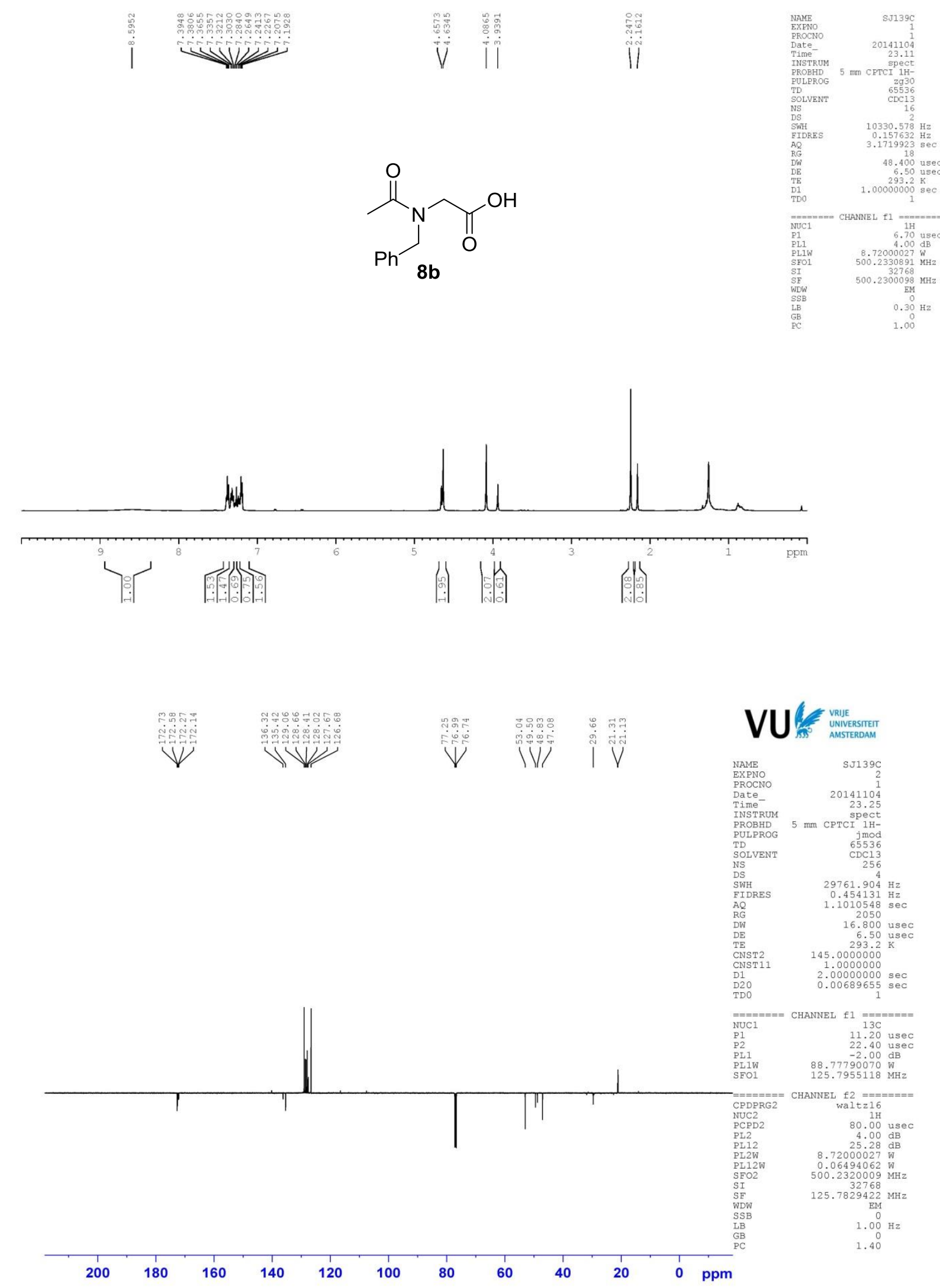

S63 

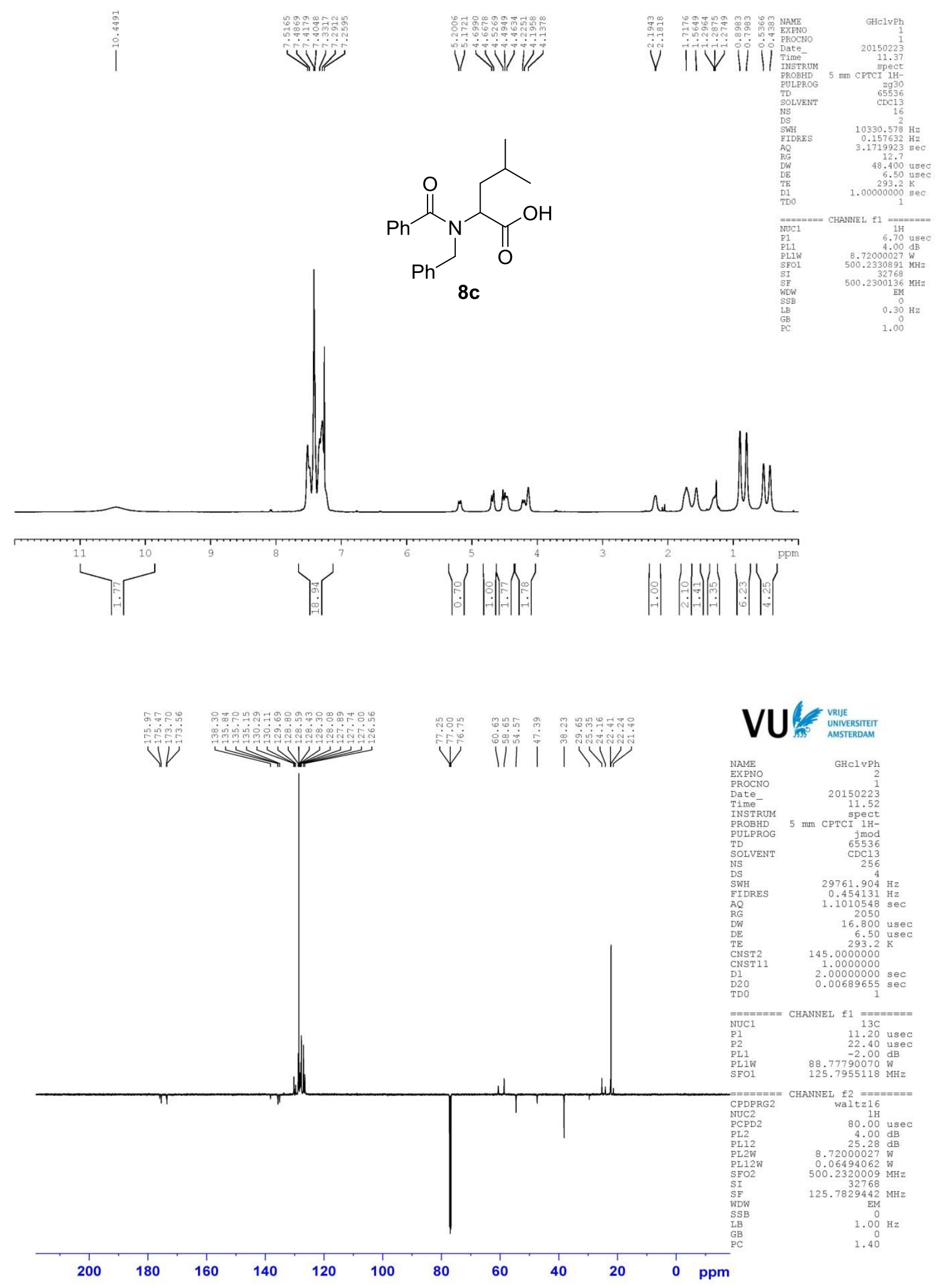

S64 

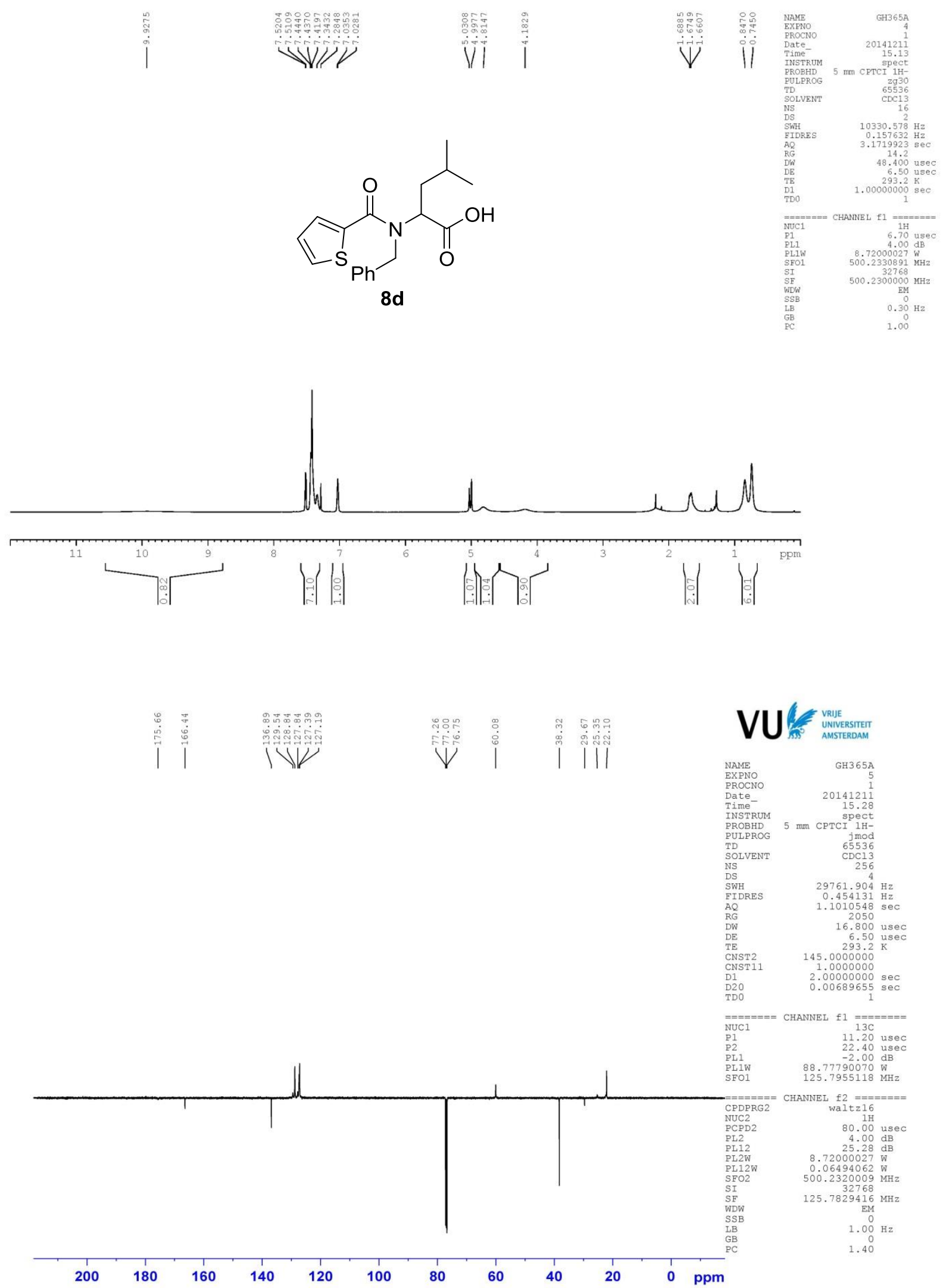

S65 


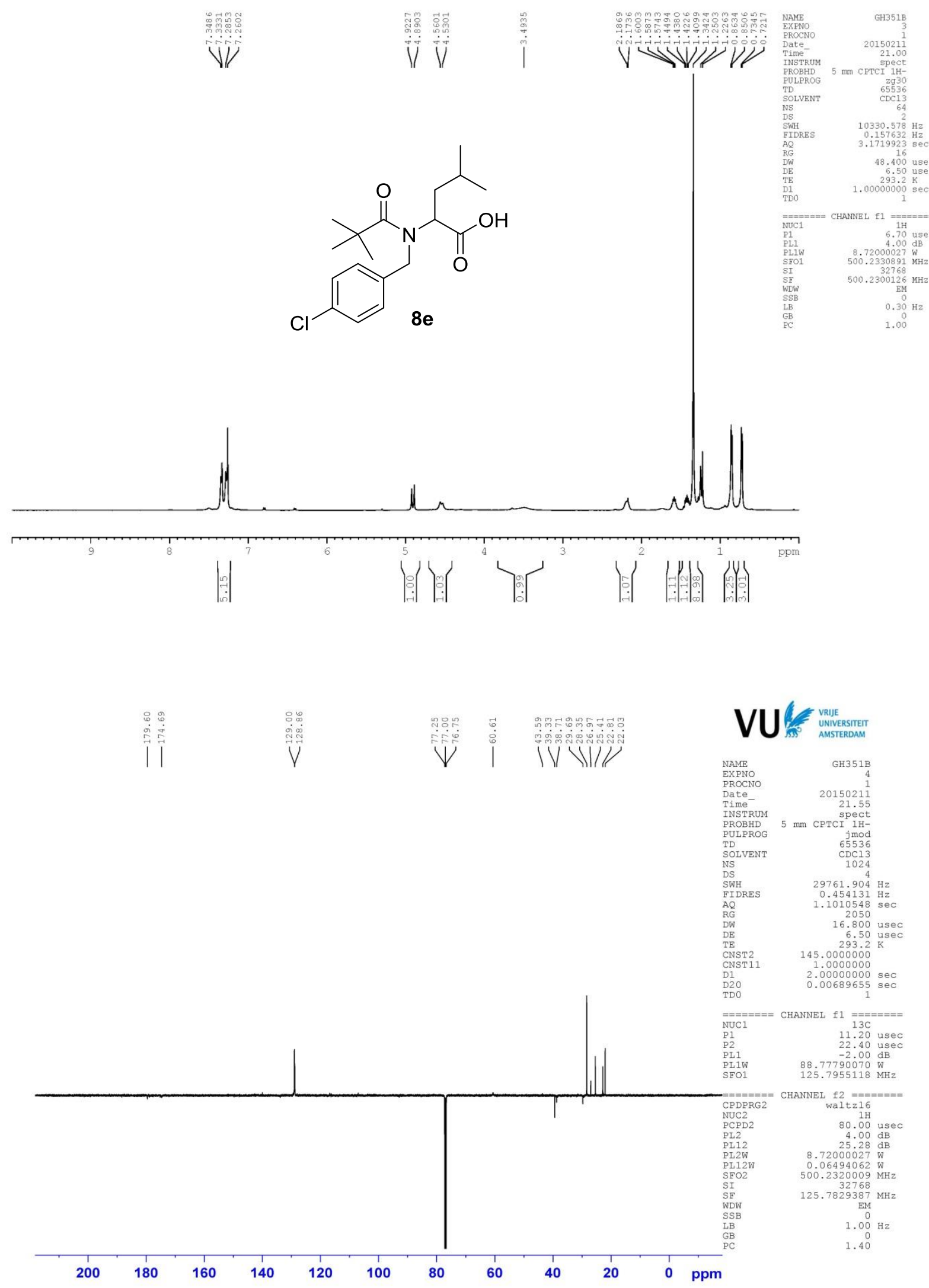




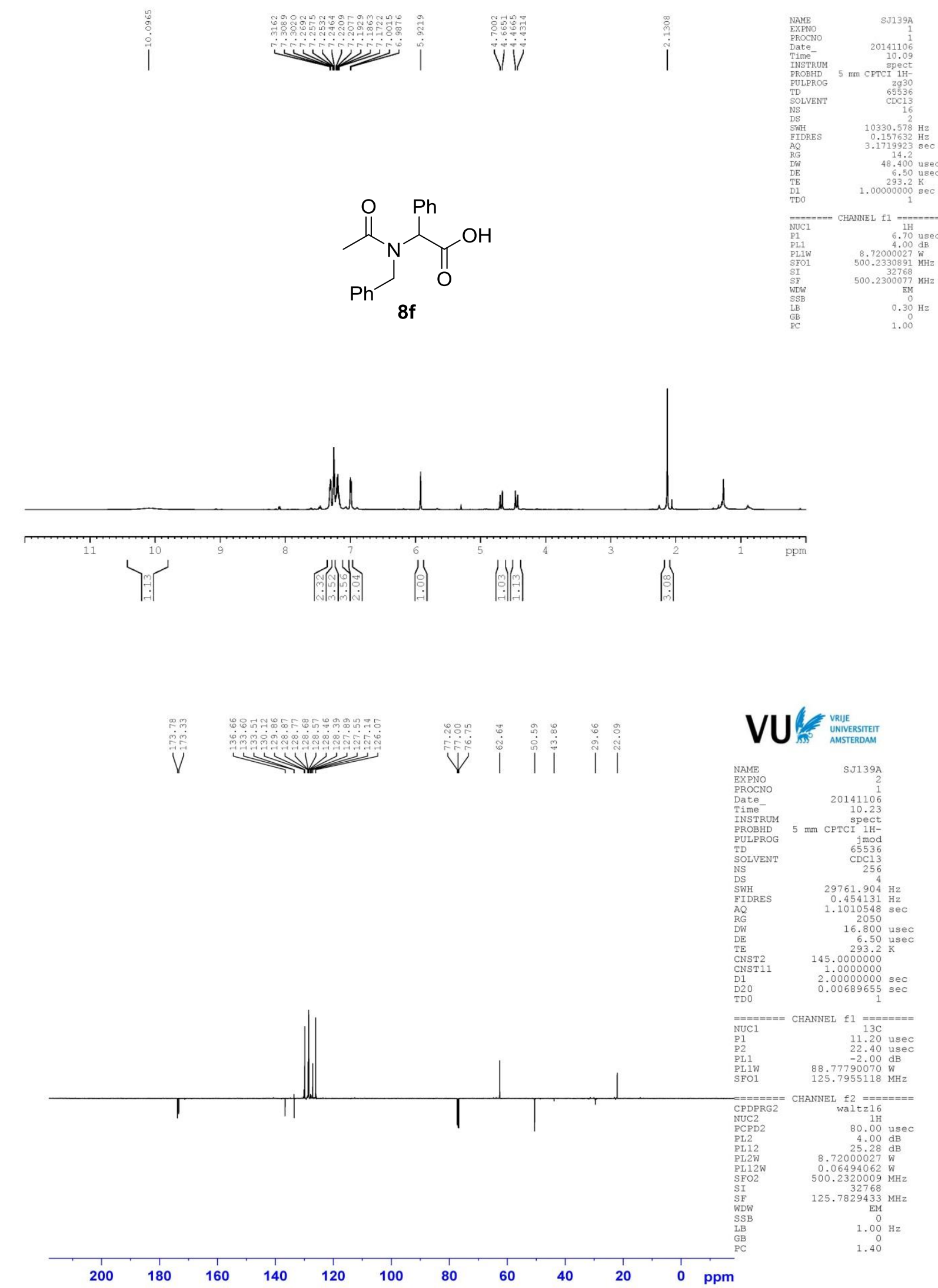

S67 

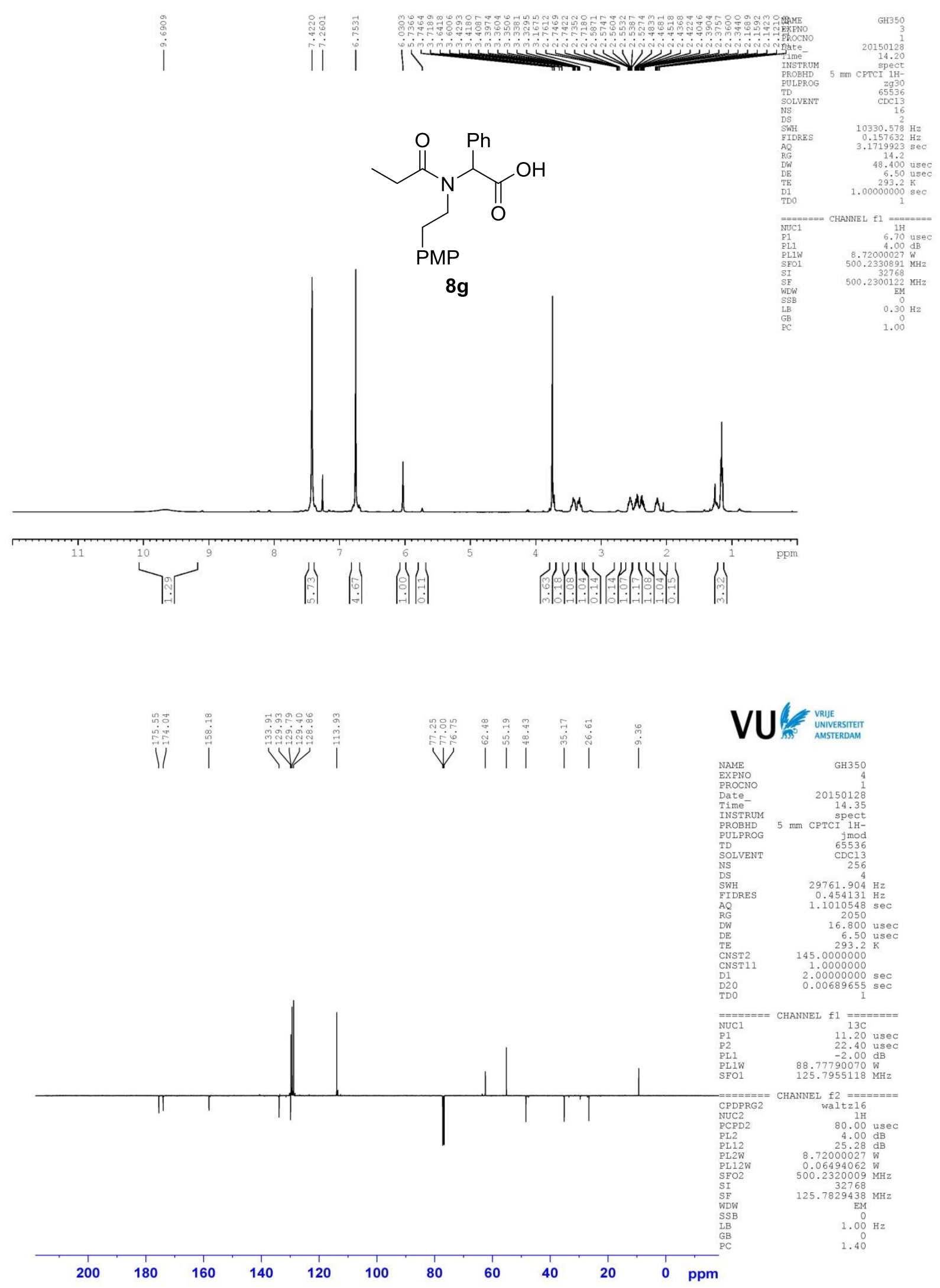

S68 


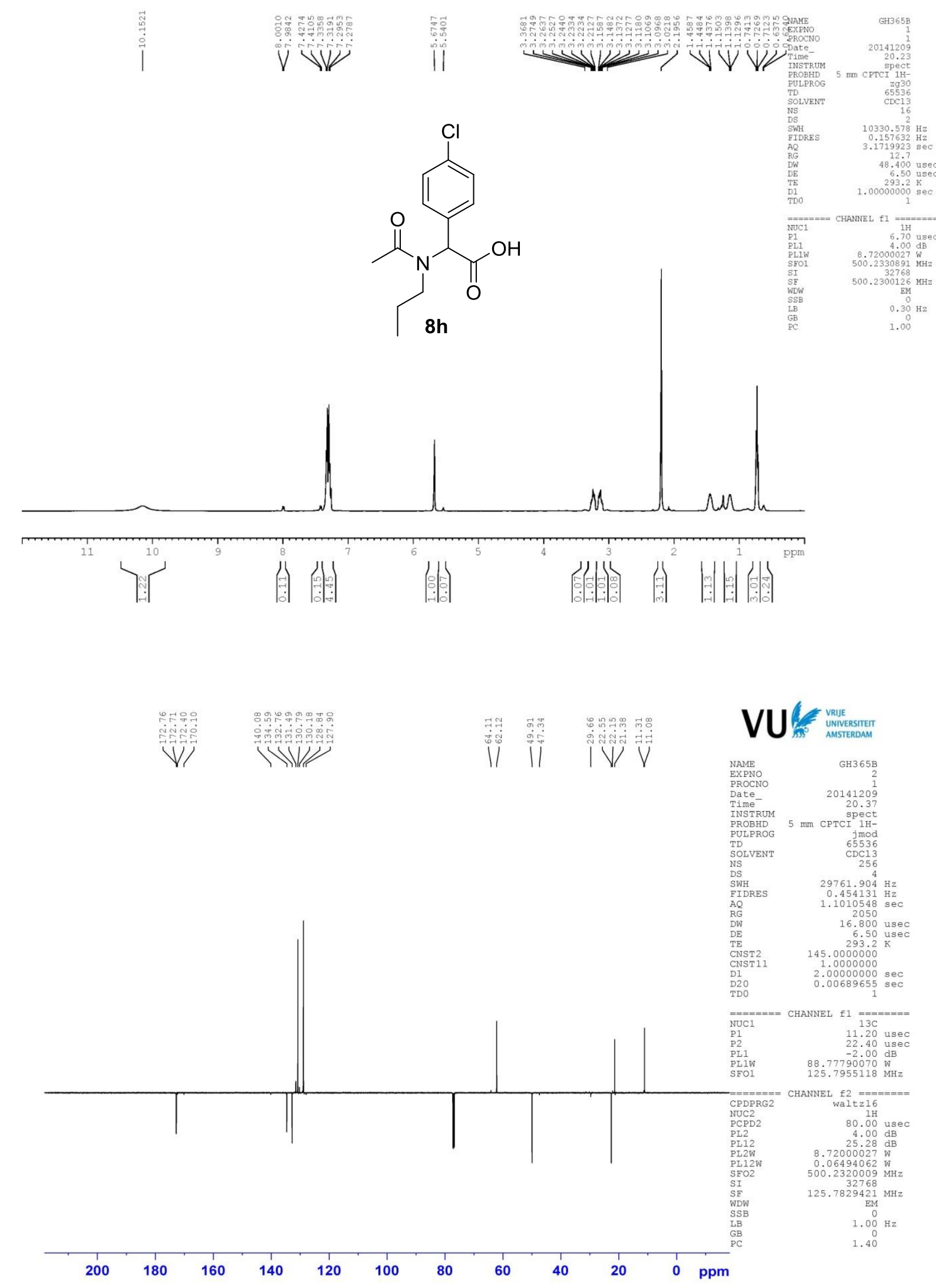

S69 


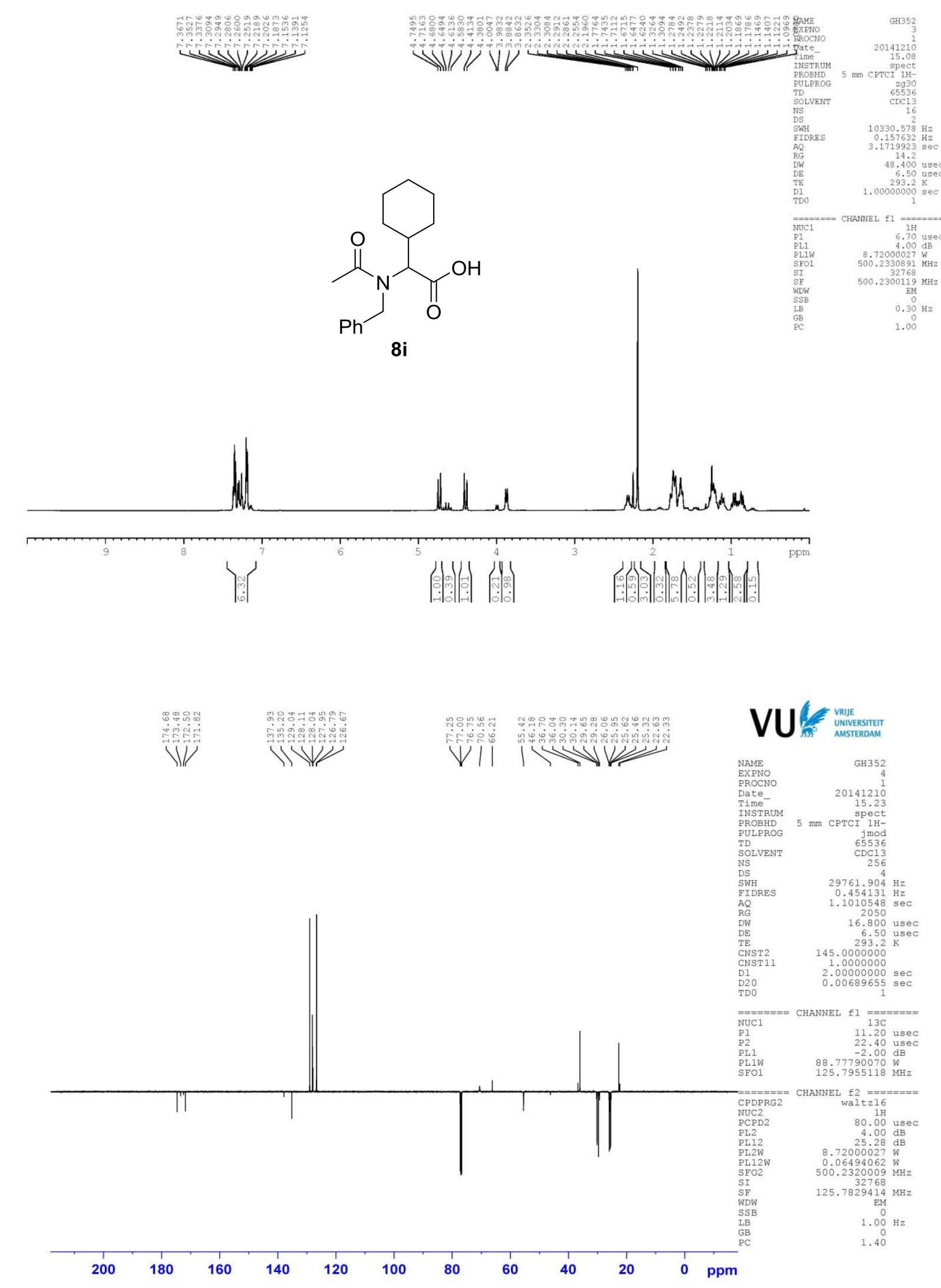



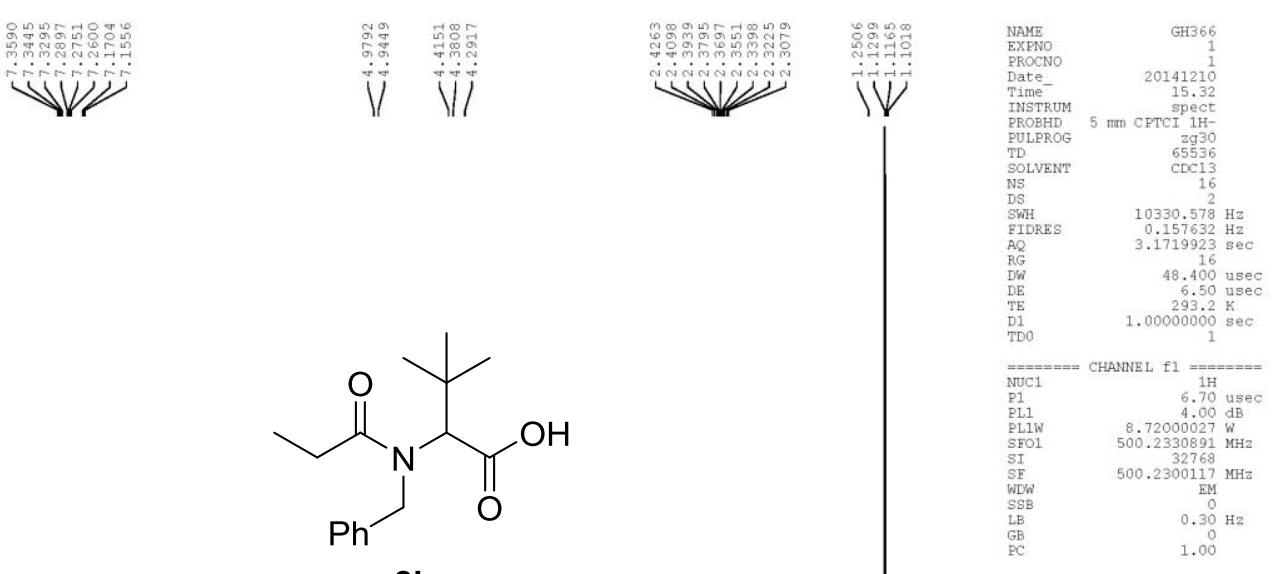

8j
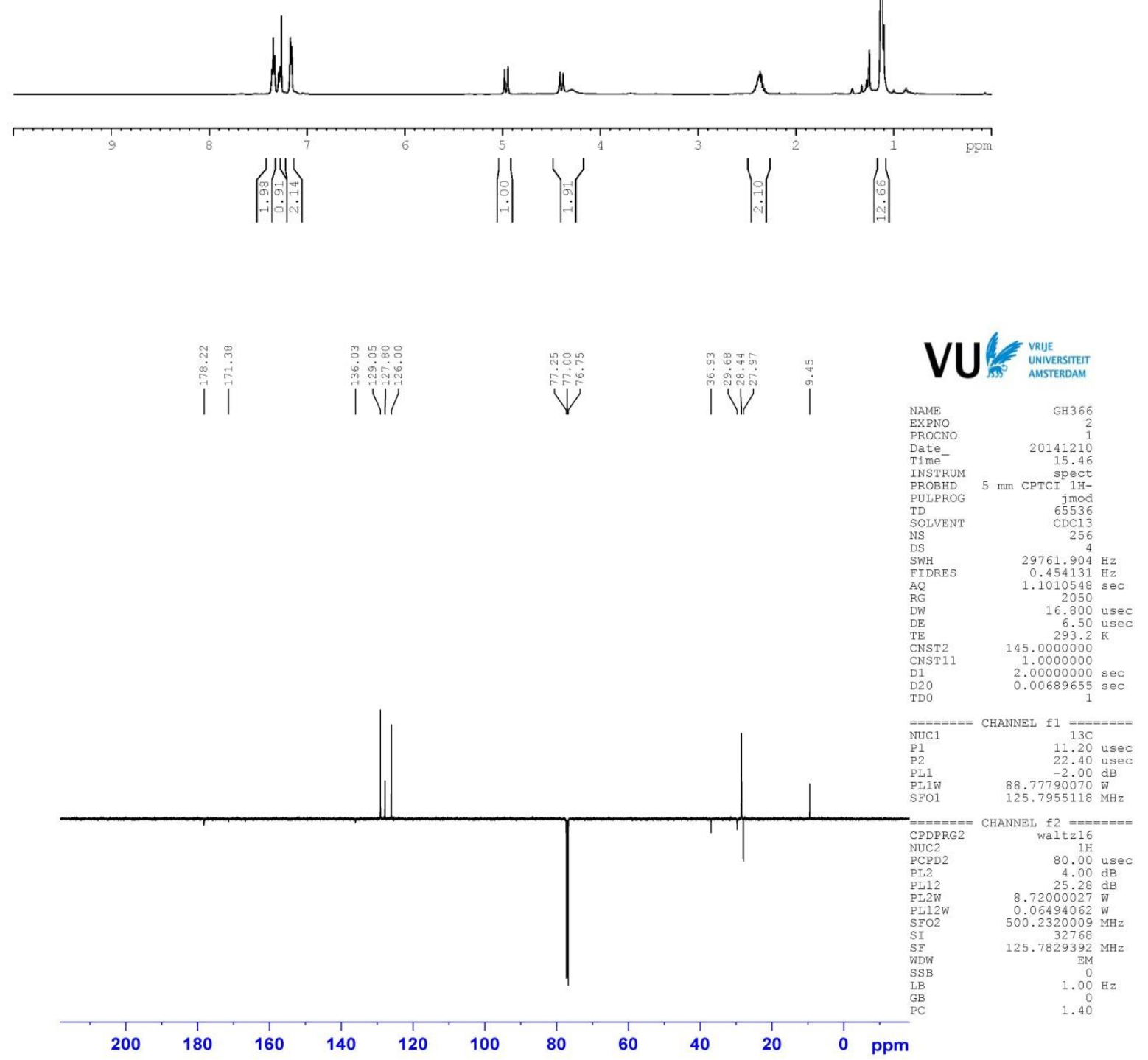

S71 

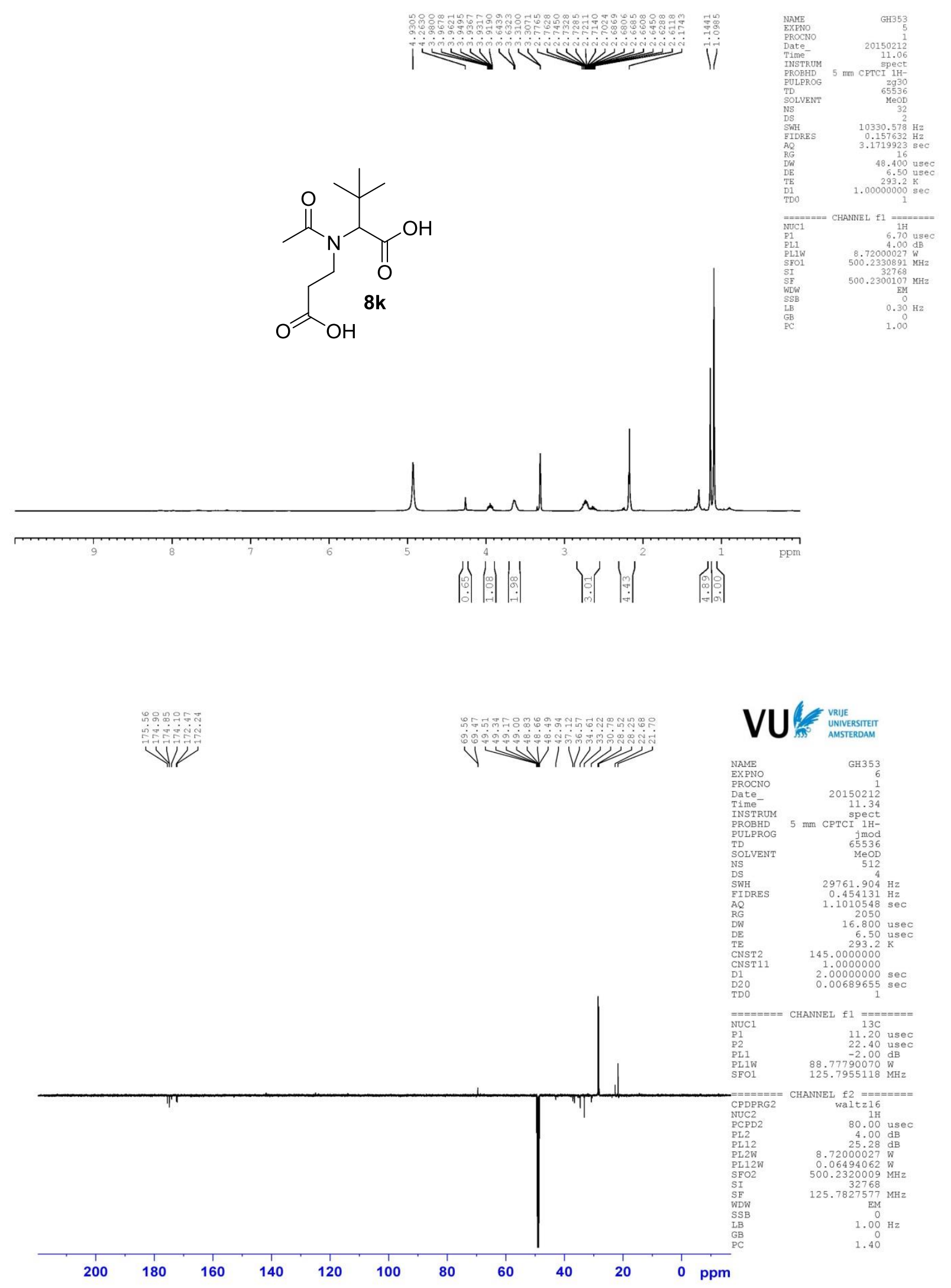


\section{Passerini products (11)}

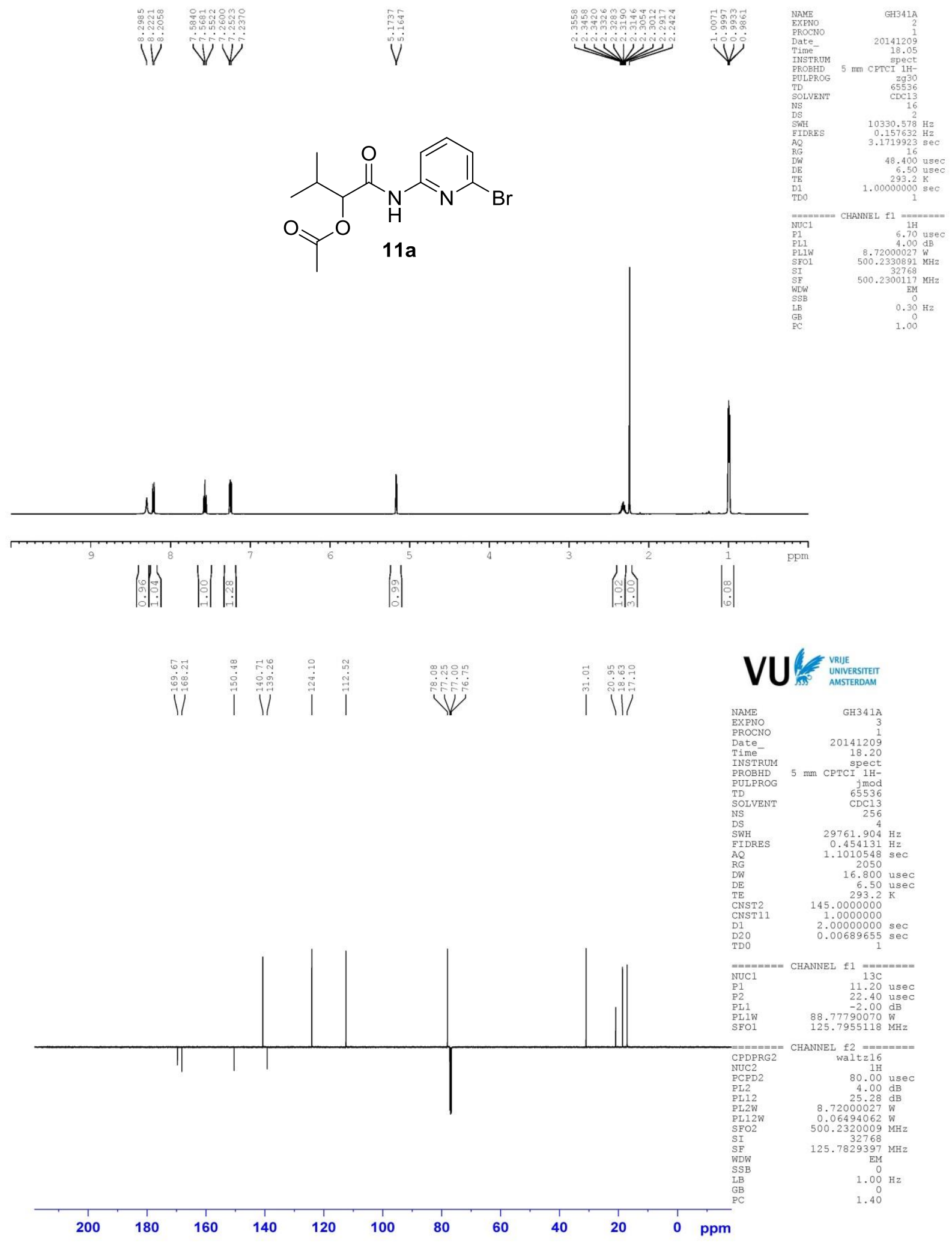




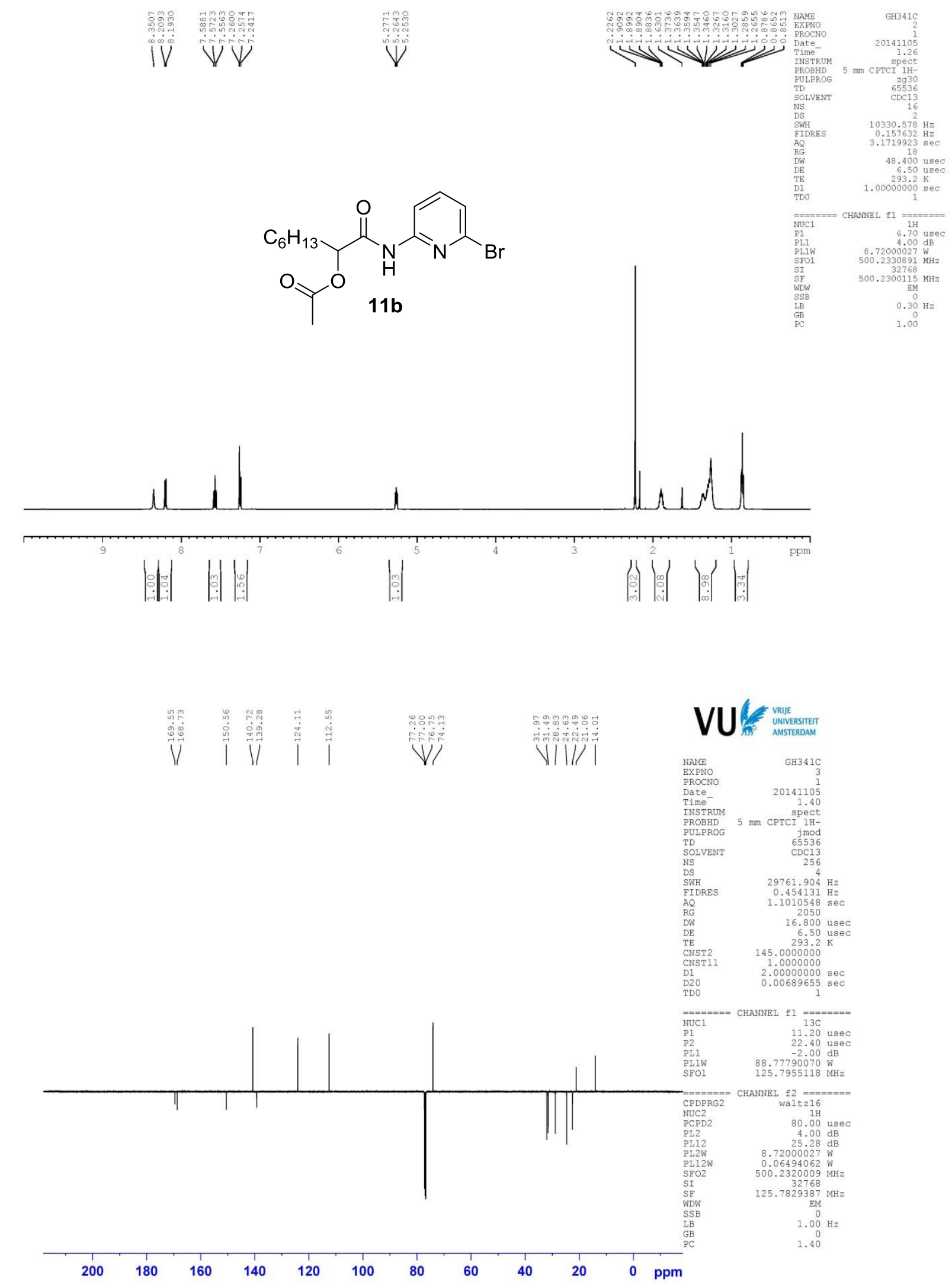


Hydrolyzed Passerini products (12)

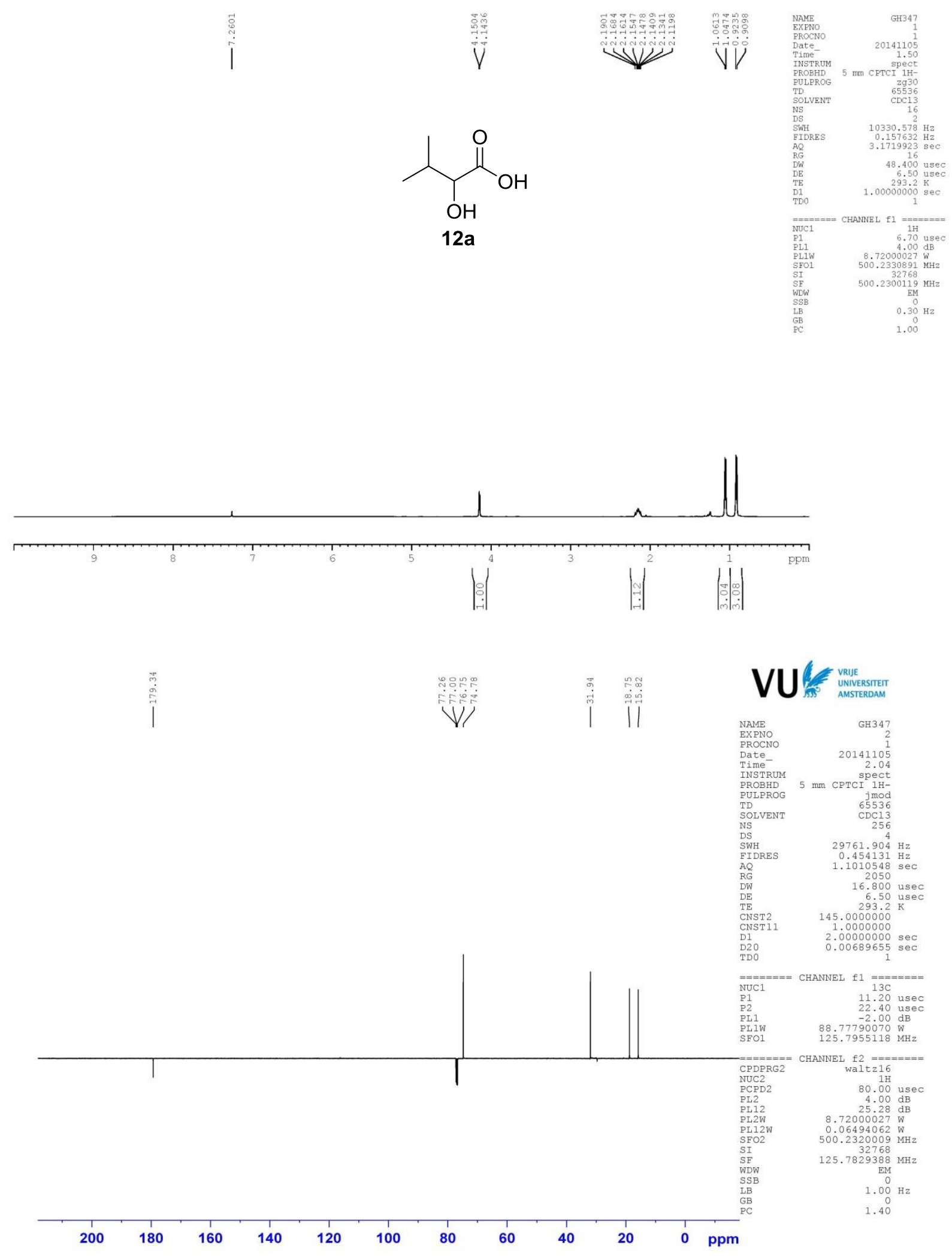




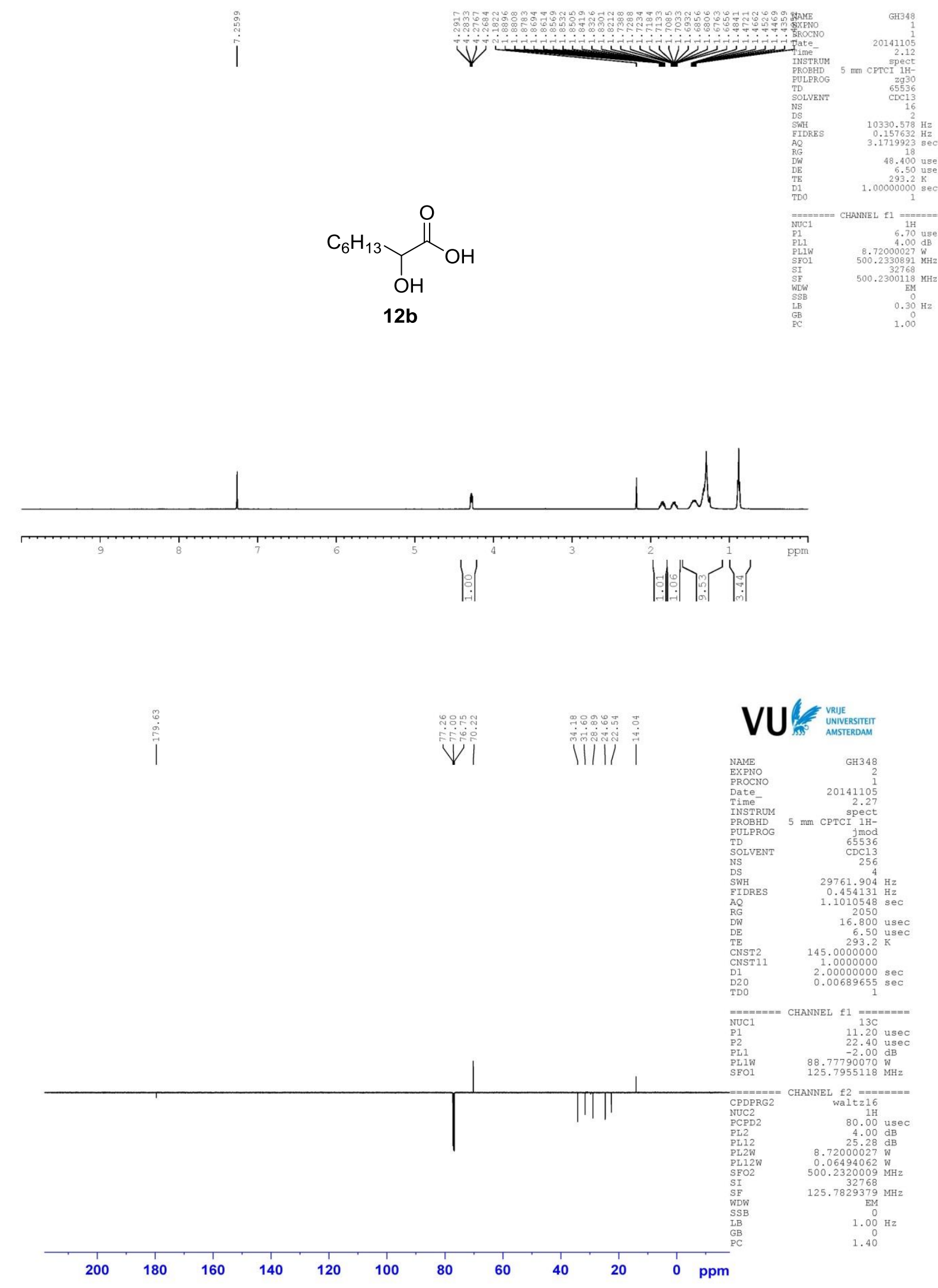

S76 
Transacylated Ugi products (10)

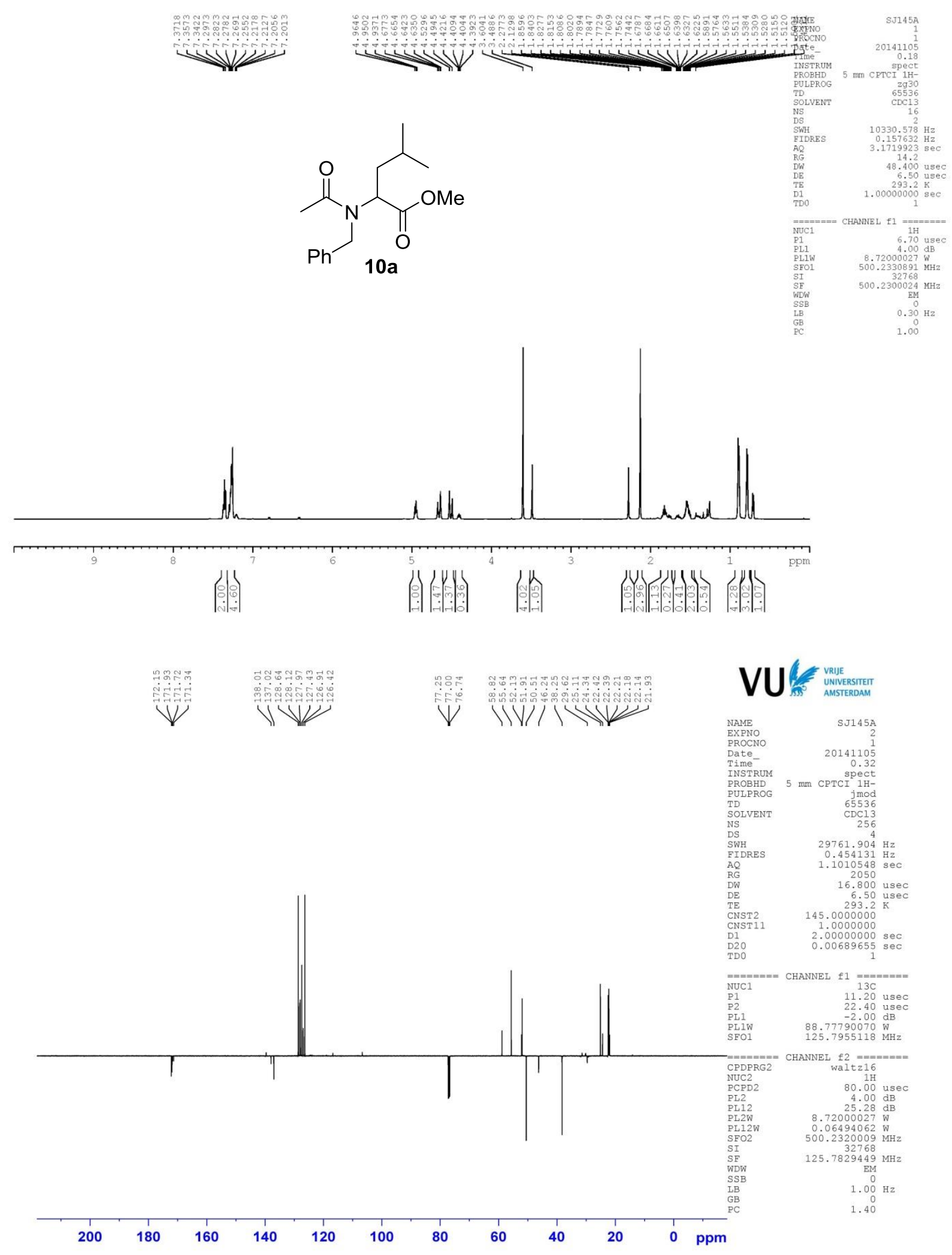




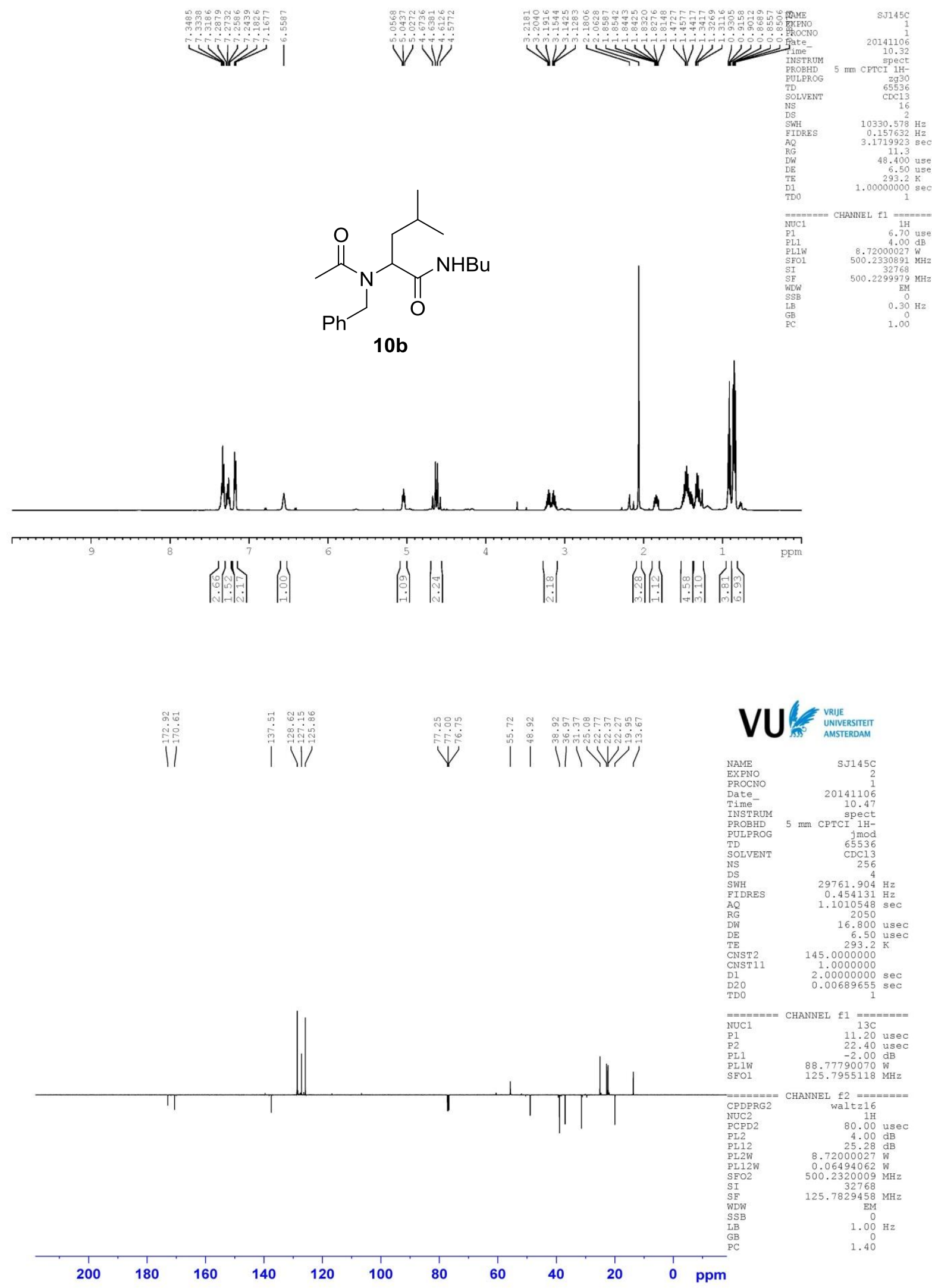



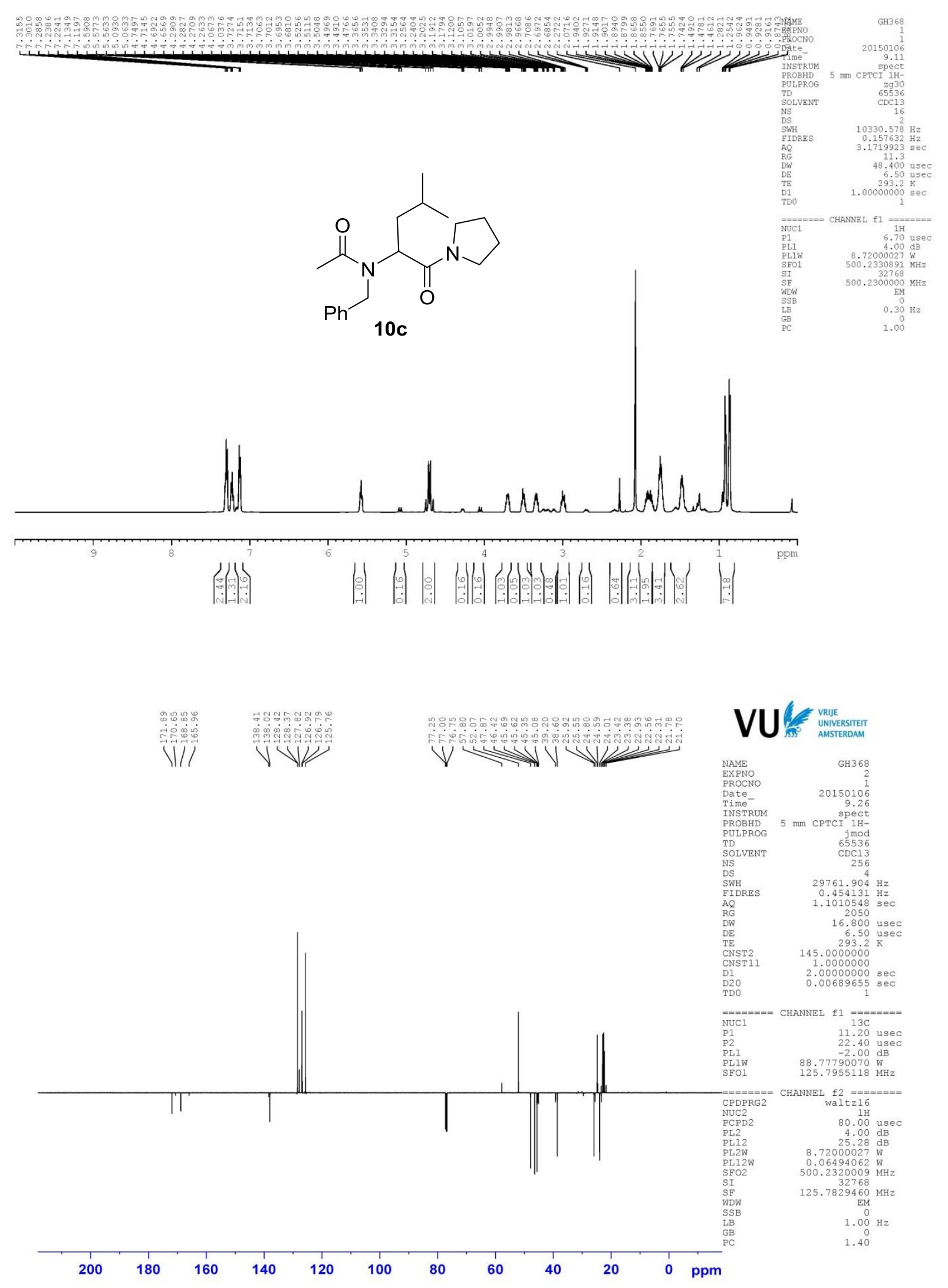

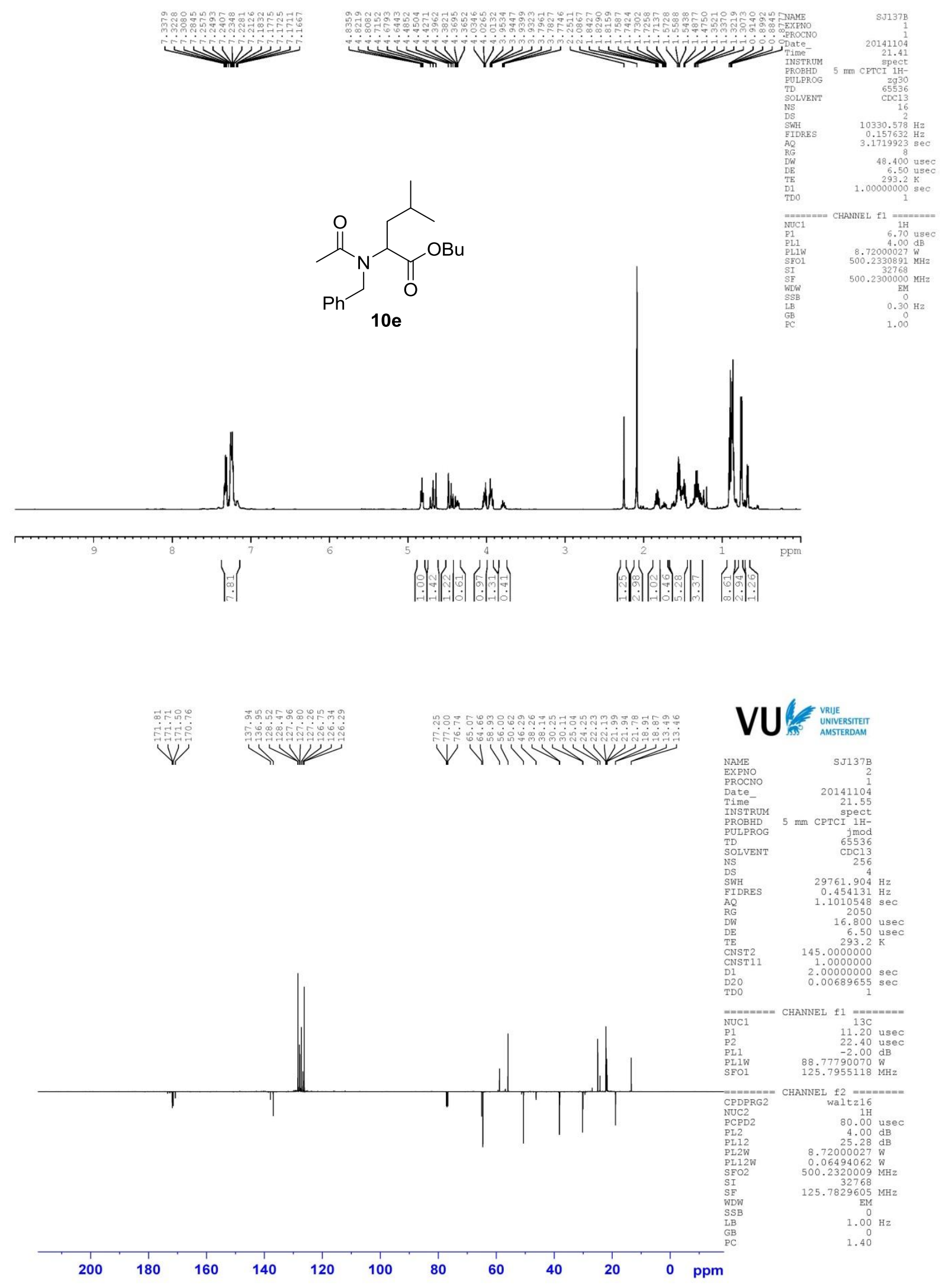

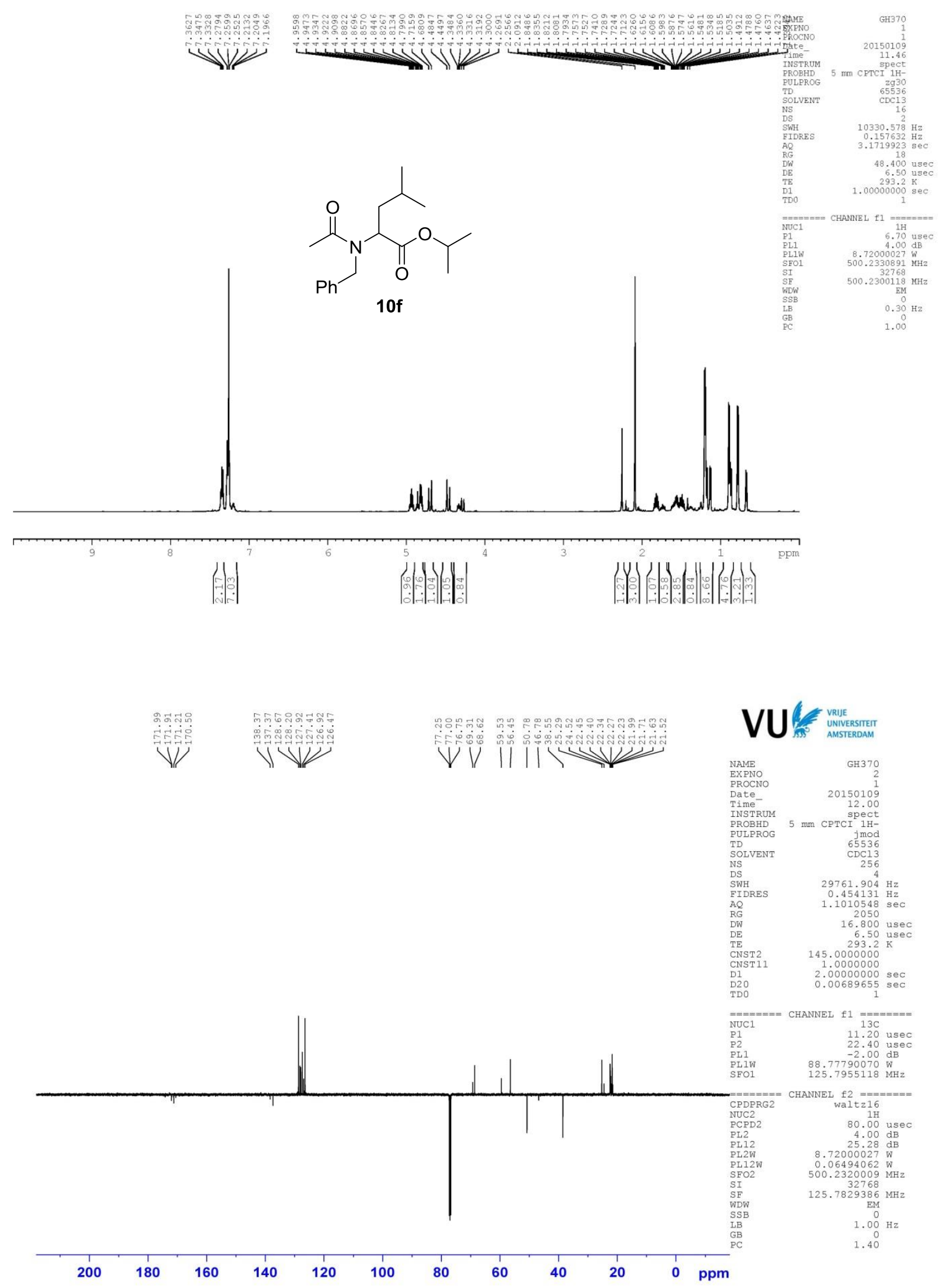

S81 

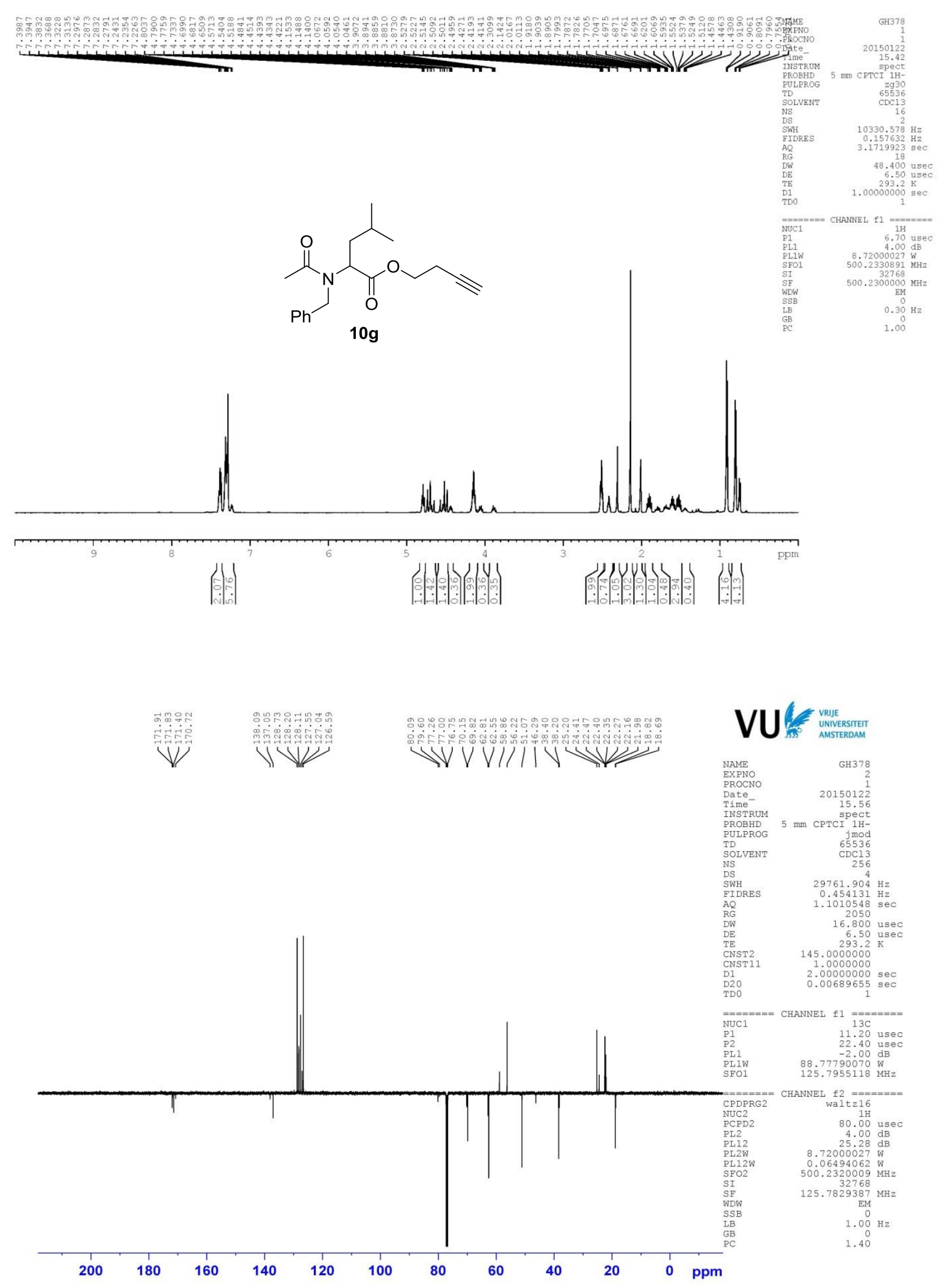

S82 

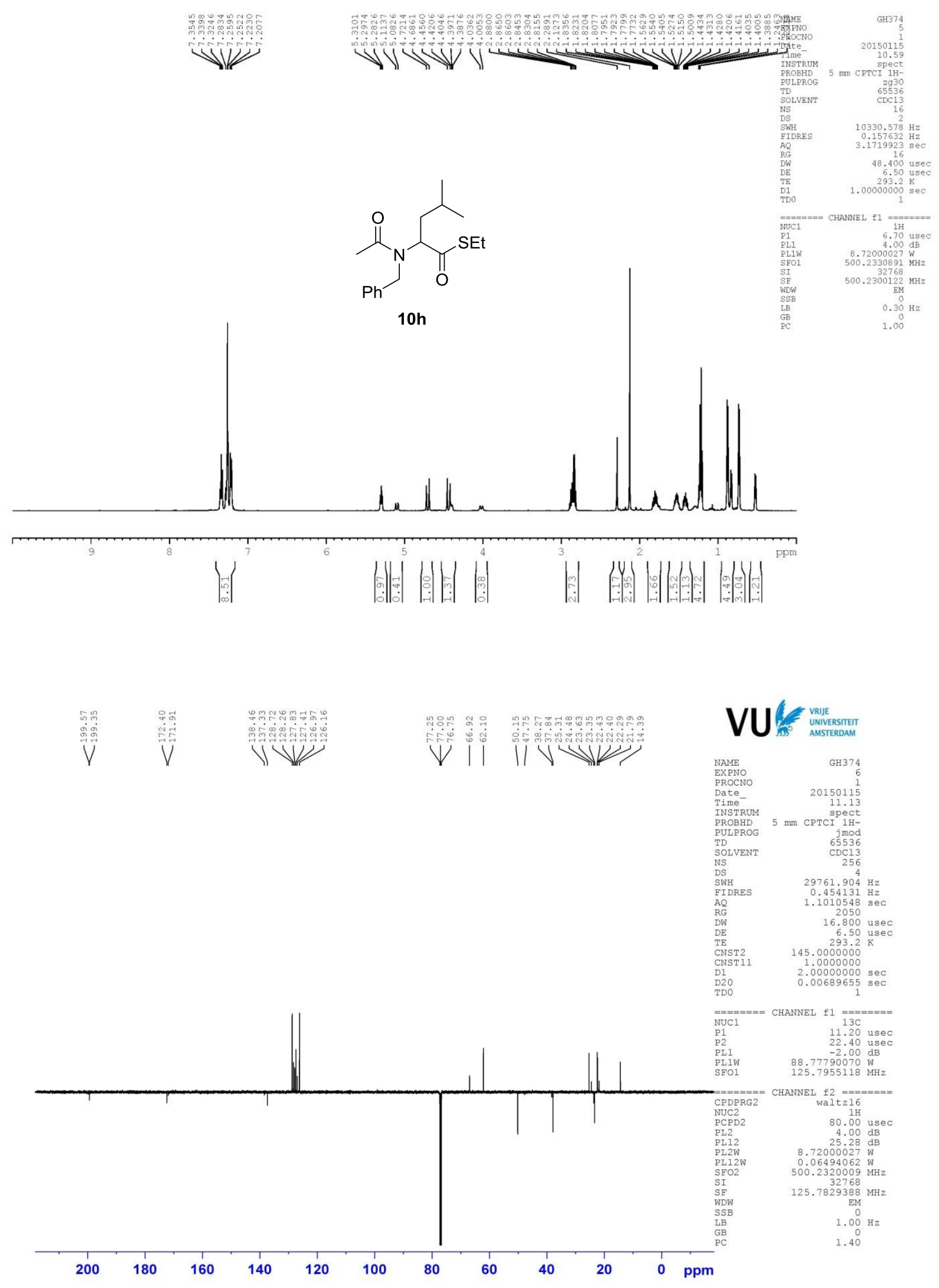

S83 


\section{Synthesis of carfentanil $(14 \& 15)$}
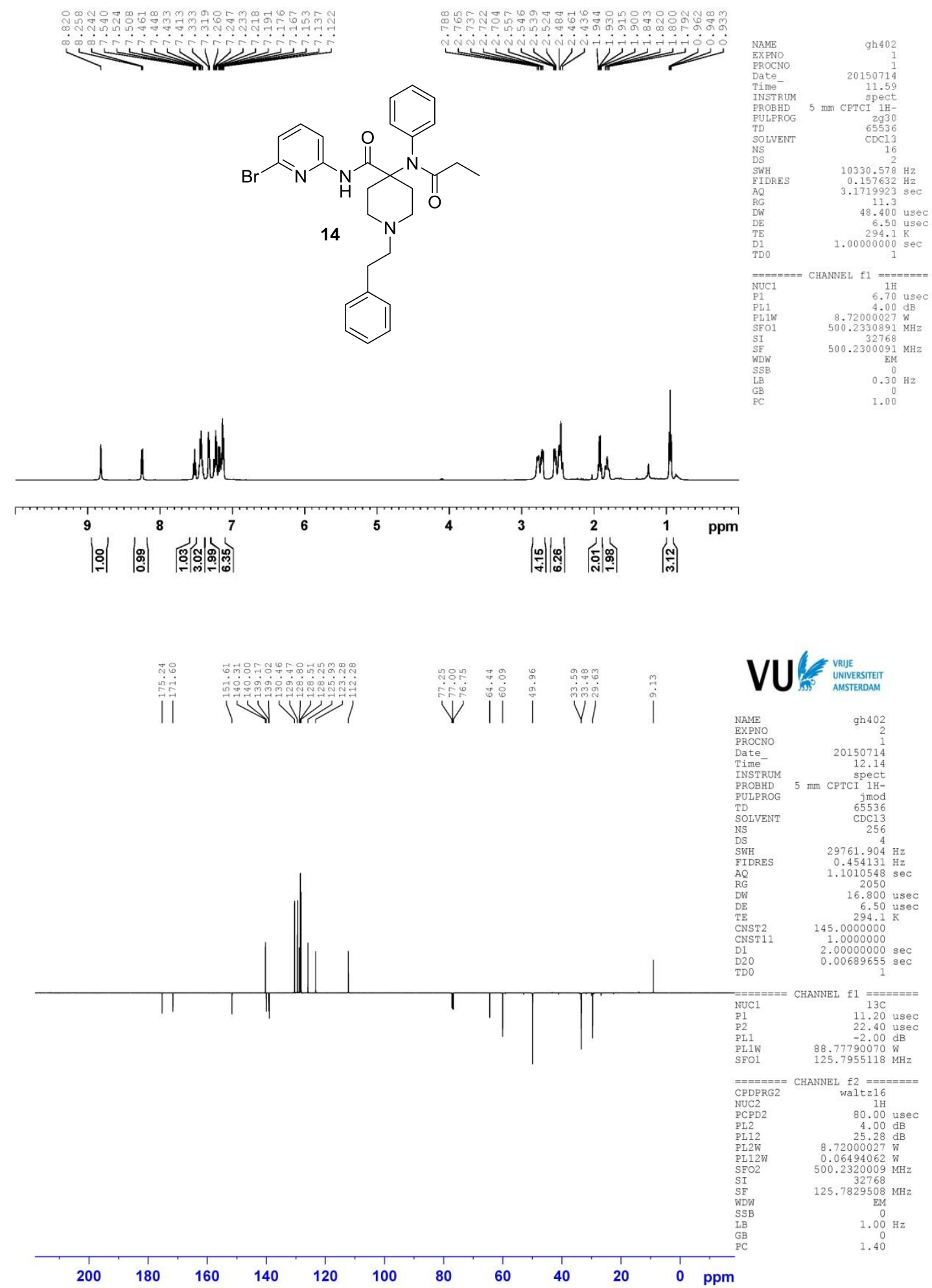

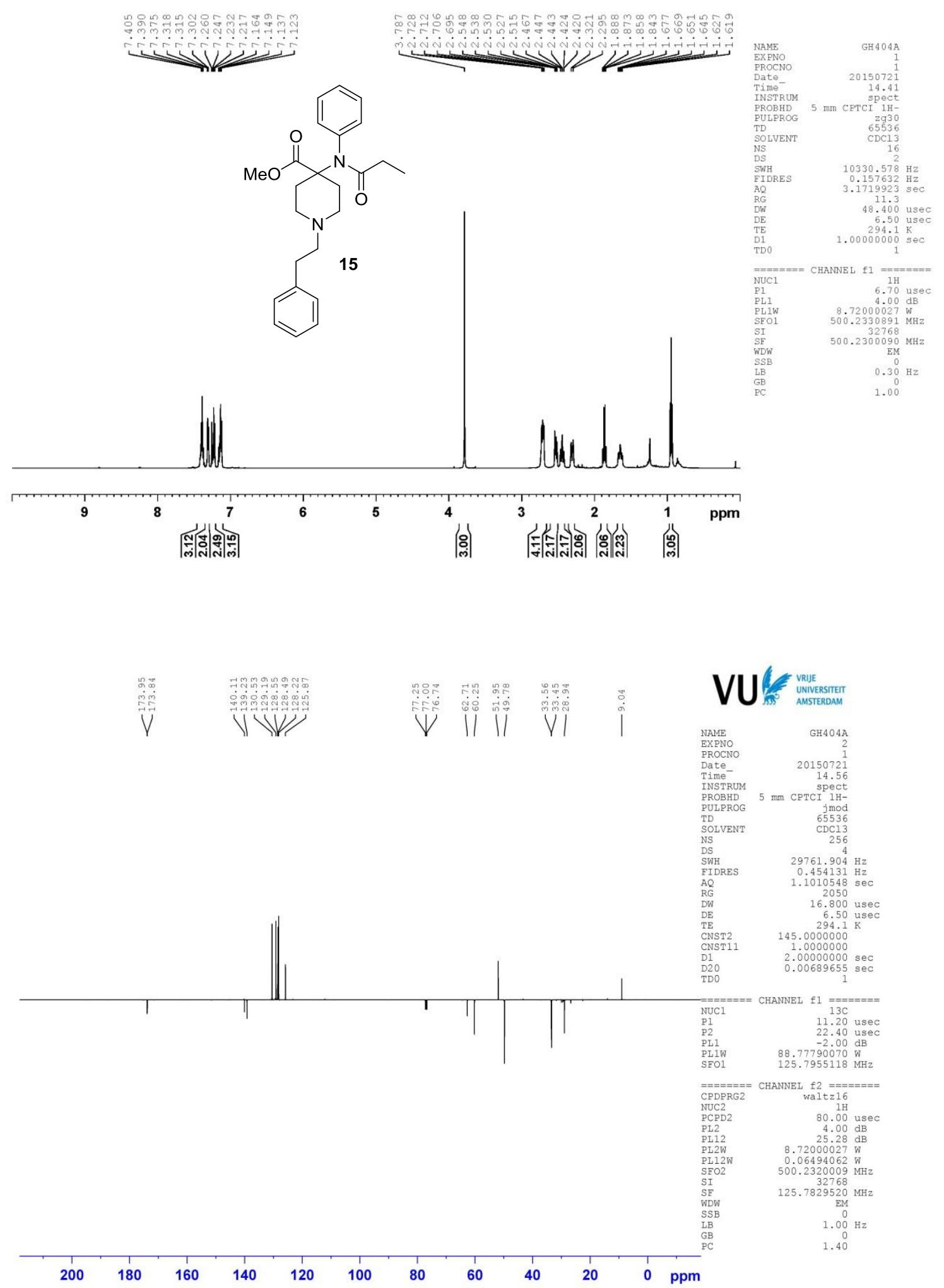